Florida International University FIU Digital Commons

\title{
Essays in Environmental Economic Valuation and Decision Making in the Presence of an Environmental Disaster
}

Jeffrey Robert Czajkowski

Florida International University, jczaj001@fiu.edu

DOI: $10.25148 /$ etd.FI08081512

Follow this and additional works at: https://digitalcommons.fiu.edu/etd

\section{Recommended Citation}

Czajkowski, Jeffrey Robert, "Essays in Environmental Economic Valuation and Decision Making in the Presence of an Environmental Disaster" (2007). FIU Electronic Theses and Dissertations. 49.

https://digitalcommons.fiu.edu/etd/49 


\section{FLORIDA INTERNATIONAL UNIVERSITY}

Miami, Florida

\section{ESSAYS IN ENVIRONMENTAL ECONOMIC VALUATION \\ AND DECISION MAKING IN THE PRESENCE OF AN \\ ENVIRONMENTAL DISASTER}

A dissertation submitted in partial fulfillment of the

requirements for the degree of

DOCTOR OF PHILOSOPHY

in

ECONOMICS

by

Jeffrey Robert Czajkowski

2007 
To: Interim Dean Mark Szuchman

College of Arts and Sciences

This dissertation, written by Jeffrey Robert Czajkowski, and entitled Essays in Environmental Economic Valuation and Decision Making in the Presence of an Environmental Disaster, having been approved in respect to style and intellectual content, is referred to you for judgment.

We have read this dissertation and recommend that it be approved.

Mahadev G. Bhat

Jonathan B. Hill

Stephen P. Leatherman

Peter Thompson, Major Professor

Date of Defense: May 30, 2007

The dissertation of Jeffrey Robert Czajkowski is approved.

Interim Dean Mark Szuchman
College of Arts and Sciences

Dean George Walker
University Graduate School

Florida International University, 2007 
C C Copyright 2007 by Jeffrey Robert Czajkowski

All rights reserved. 


\section{DEDICATION}

I dedicate this dissertation to my parents who instilled in me the value of an education. Thank you Mom and Dad for all of your sacrifices and unwavering support toward my education! 


\section{ACKNOWLEDGMENTS}

The road to the completion of my Ph.D. has not been easy, but is one that I am happy I have taken. First and foremost I want to thank my advisor and committee chair, Peter Thompson. Thank you for fighting for me, guiding me, pushing me, tolerating me, listening to me, and promoting me along the way. I know that I am a better economist and person due to our interaction, and I hope that I can mentor future students with the same patience and enthusiasm that you have shown to me.

I also certainly need to thank the other members of my committee. I have known Mahadev Bhat since the beginning of my graduate education at FIU, and I am very grateful for all of the time, guidance, opportunities, and encouragement he has provided along the way. He has been a steadfast advocate of my interests. Jonathan Hill has been instrumental in focusing me on doing quality economic research, while also being an esteemed source of input and encouragement on many different levels. Lastly, I am very appreciative to Stephen Leatherman for his flexibility toward, and enthusiastic interest in my research.

Additionally, I need to thank all of the faculty and staff of the economics department. I want to specifically thank John Boyd, Cem Karayalcin, and Mariela Delgado for going out of their way to help me in numerous instances during my time at FIU. I am also very grateful for financial support provided to me through the U.S. E.P.A STAR Fellowship, FIU's International Hurricane Research Center, and FIU's University Graduate School Dissertation Year Fellowship.

Lastly, special thanks go to my "combo" - Cynthia, Arami, and Jordi - you have meant more to me this last year than you will ever know. 


\title{
ABSTRACT OF THE DISSERTATION
}

\section{ESSAYS IN ENVIRONMENTAL ECONOMIC VALUATION AND DECISION \\ MAKING IN THE PRESENCE OF AN ENVIRONMENTAL DISASTER}

\author{
by \\ Jeffrey Robert Czajkowski \\ Florida International University, 2007 \\ Miami, Florida \\ Professor Peter Thompson, Major Professor
}

The first essay developed a respondent model of Bayesian updating for a doublebound dichotomous choice (DB-DC) contingent valuation methodology. I demonstrated by way of data simulations that current DB-DC identifications of true willingness-to-pay (WTP) may often fail given this respondent Bayesian updating context. Further simulations demonstrated that a simple extension of current DB-DC identifications derived explicitly from the Bayesian updating behavioral model can correct for much of the WTP bias. Additional results provided caution to viewing respondents as acting strategically toward the second bid. Finally, an empirical application confirmed the simulation outcomes.

The second essay applied a hedonic property value model to a unique water quality (WQ) dataset for a year-round, urban, and coastal housing market in South Florida, and found evidence that various WQ measures affect waterfront housing prices in this setting. However, the results indicated that this relationship is not consistent across any of the six particular WQ variables used, and is furthermore dependent upon the specific descriptive statistic employed to represent the WQ measure in the empirical 
analysis. These results continue to underscore the need to better understand both the WQ measure and its statistical form homebuyers use in making their purchase decision.

The third essay addressed a limitation to existing hurricane evacuation modeling aspects by developing a dynamic model of hurricane evacuation behavior. A household's evacuation decision was framed as an optimal stopping problem where every potential evacuation time period prior to the actual hurricane landfall, the household's optimal choice is to either evacuate, or to wait one more time period for a revised hurricane forecast. A hypothetical two-period model of evacuation and a realistic multi-period model of evacuation that incorporates actual forecast and evacuation cost data for my designated Gulf of Mexico region were developed for the dynamic analysis. Results from the multi-period model were calibrated with existing evacuation timing data from a number of hurricanes. Given the calibrated dynamic framework, a number of policy questions that plausibly affect the timing of household evacuations were analyzed, and a deeper understanding of existing empirical outcomes in regard to the timing of the evacuation decision was achieved. 


\section{TABLE OF CONTENTS}

CHAPTER

PAGE

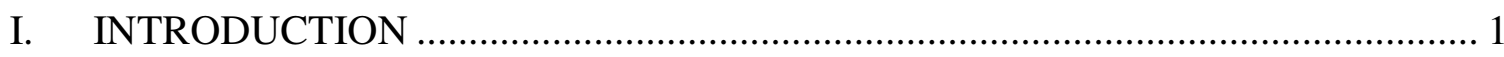

II. WILLINGNESS-TO-PAY BIAS IN DICHOTOMOUS CHOICE SURVEYS....... 10

II.I. Introduction............................................................................... 10

II.II. Respondent Bayesian Updating Model..................................................... 13

II.III. Identification of True WTP.............................................................. 15

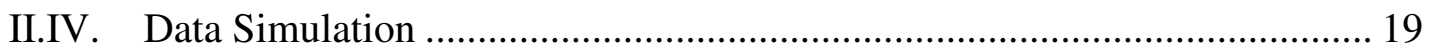

II.V. Estimation and Results......................................................................... 21

II.VI. Empirical Application ........................................................................ 29

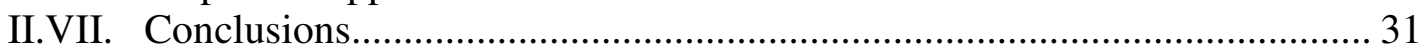

III. DOES WATER QUALITY MATTER TO SOUTH FLORIDA HOMEBUYERS?

EVIDENCE FROM A HEDONIC ANALYSIS................................................ 41

III.I. Introduction .................................................................................... 41

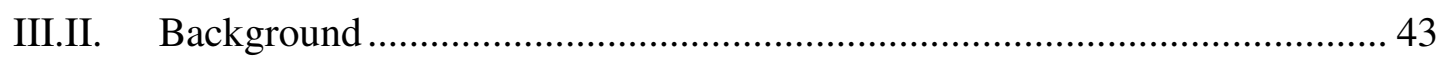

III.III. Study Area and Associated Data................................................................ 46

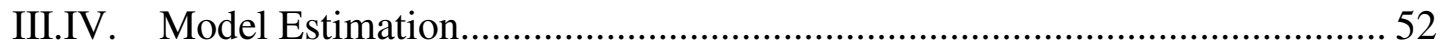

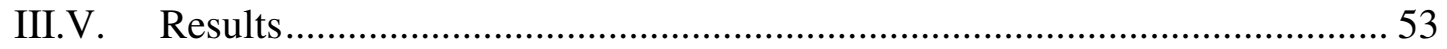

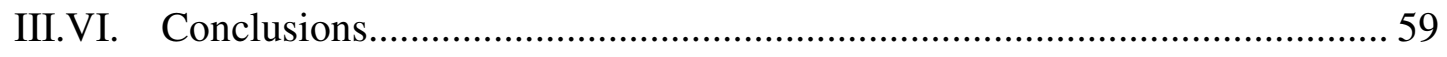

IV. IS IT TIME TO GO YET? DYNAMICALLY MODELING HURRICANE

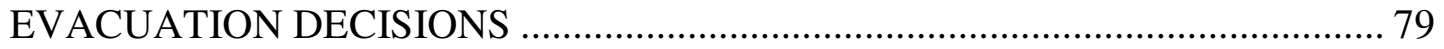

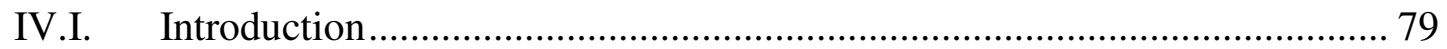

IV.II. The Uncertain Nature of Hurricane Forecasts and Evacuation Timing....... 83

IV.III. A Two-Period Model of Hurricane Evacuation............................................. 97

IV.IV. A Multi-Period Model of Evacuation .................................................... 118

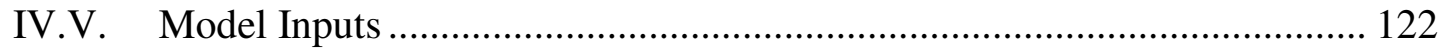

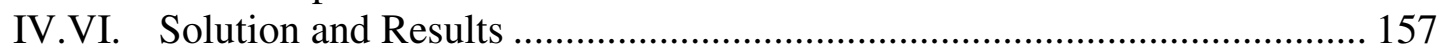

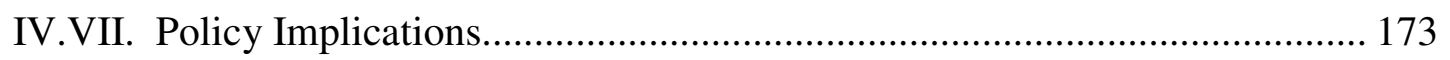

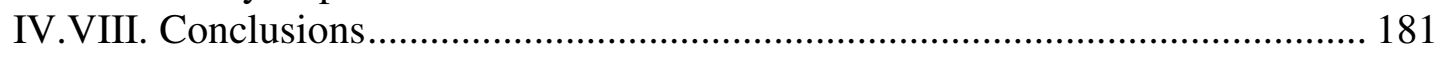

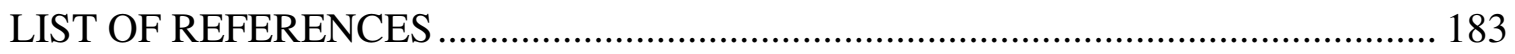

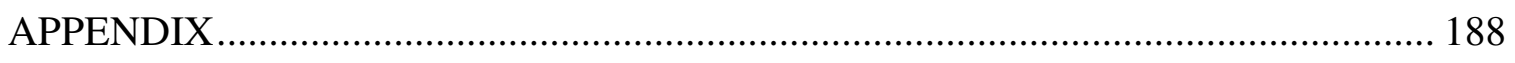

VITA ………… 


\section{LIST OF TABLES}

TABLE

PAGE

II.1. Specified Values for $\bar{\theta}_{\mathrm{i}}, \mu_{\mathrm{i}}, \sigma_{\theta}{ }^{2}, \alpha_{\mathrm{ij}}, \sigma_{\varepsilon_{\mathrm{ij}}}{ }^{2}$, and $\mathrm{b}_{\mathrm{ij}}$ Used In the Data Simulation............... 32

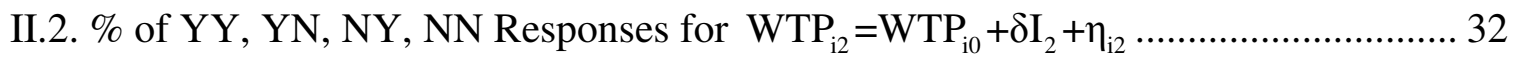

II.3. $\delta$ Coefficient Results For Halving/Doubling, And (+)/(-) $60 b_{\mathrm{i} 2}$ Generation........... 33

II.4: Alaska Study Maximum Likelihood Estimates................................................. 33

III.1. Waterfront Sales by Month from Jan 2000 to Aug 2004 ..................................... 60

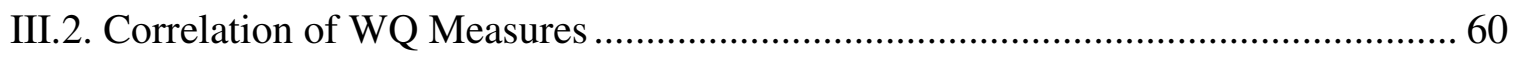

III.3. DO Levels Statistical Descriptors by Month for Location 2. North Fork .............. 61

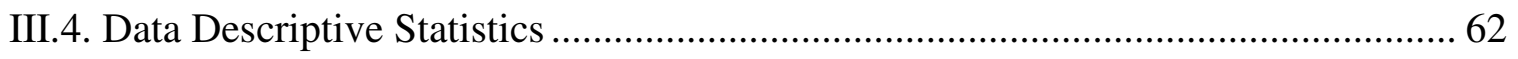

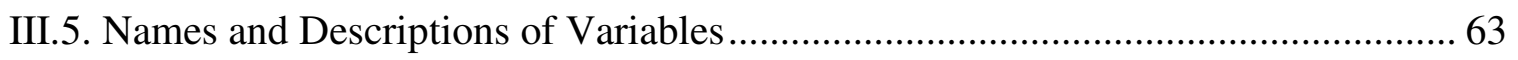

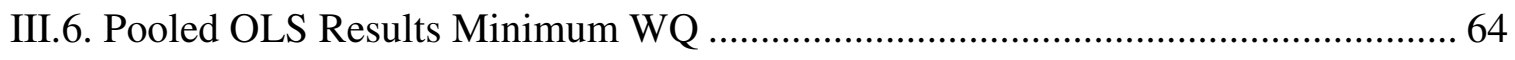

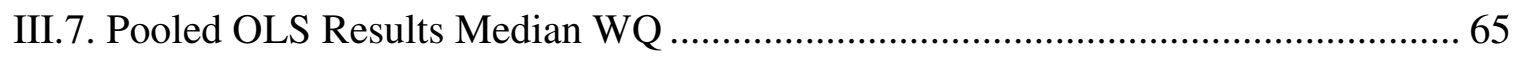

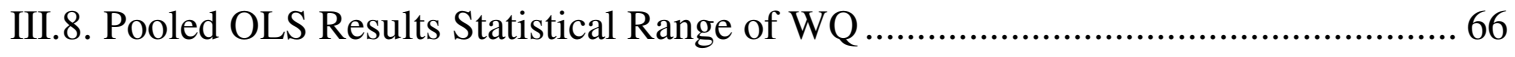

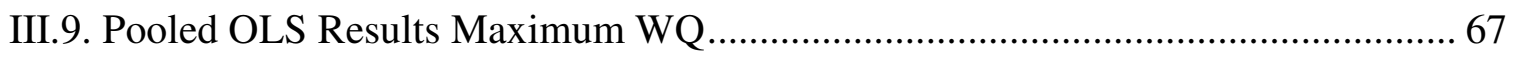

III.10. Pooled WQ Measure Results by Statistical Descriptor..................................... 68

III.11. Pooled WQ Measure Hedonic Prices by Statistical Descriptor .......................... 68

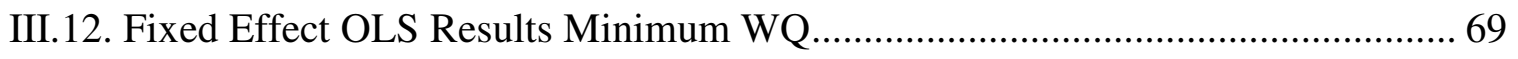

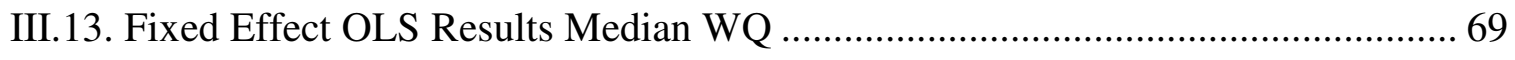

III.14. Fixed Effect OLS Results Statistical Range of WQ ...................................... 70

III.15. Fixed Effect OLS Results Maximum WQ .................................................... 70

III.16. Fixed Effect WQ Measure Results by Statistical Descriptor.............................. 71 
IV.1. Saffir-Simpson Hurricane Scale . 87

IV.2. 15 Coastal Gulf of Mexico Locations - County/Parish (Nearest Major City) ...... 125

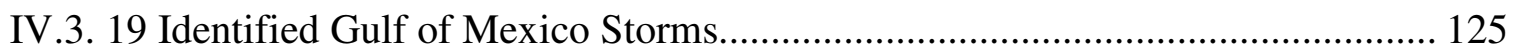

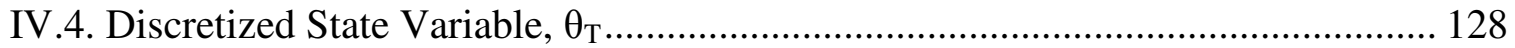

IV.5. Average NHC 10-Year Intensity Forecast Errors (1996-2005)............................. 129

IV.6. Construction of Risk Indices, Steps 1) to 3) .................................................. 130

IV.7. Hurricane Dennis $\left(\mathrm{T}^{*}\right.$-n) Forecasted Landfall Points.............................................. 133

IV.8. Pensacola, FL Risk Indices for Hurricane Dennis................................................. 135

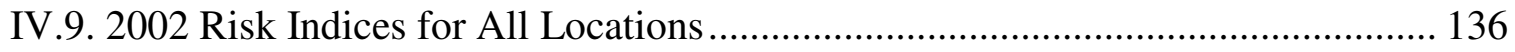

IV.10. 2002 T* to T Aggregate Transition Matrix ………………................................ 137

IV.11. Markov Transition Matrix for T* to T............................................................ 138

IV.12. Average Costs of Evacuation (2004 dollars) ................................................... 141

IV.13. Cumulative Evacuation Timing Summary ...................................................... 148

IV.14. Increasing/Decreasing Evacuation Costs...................................................... 150

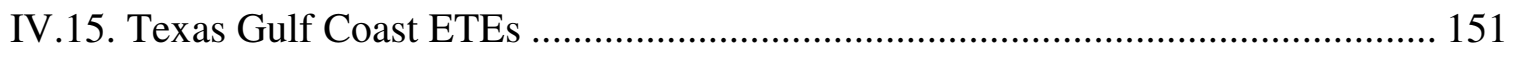

IV.16. Derived CAT 1 to CAT 5 Costs of Evacuation ................................................. 152

IV.17. Cost of Injuries (Source MMC) …………………..................................... 155

IV.18. Expected Costs of Not Evacuating ................................................................ 157

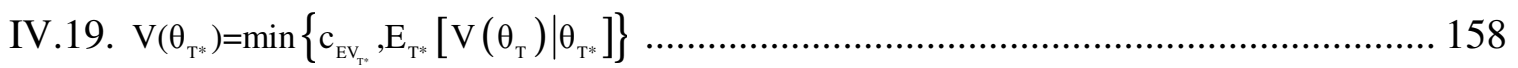

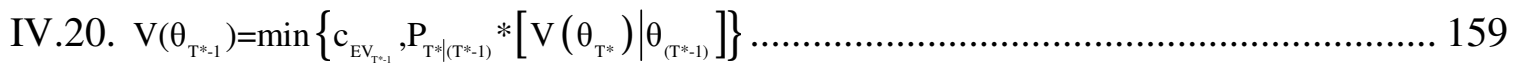

IV.21. Hurricane Ivan Risk Indices by Location ......................................................... 162

IV.22. Hurricane Opal Risk Indices by Location .......................................................... 164 
IV .23. Hurricane Charley Risk Indices by Location................................................. 165

IV.24. Hurricane Lili Risk Indices by Location ...................................................... 167 


\section{LIST OF FIGURES}

FIGURE

PAGE

II.1. An Example of Generated Yes/No Responses ................................................. 34

II.2. Simulation results for $\mathrm{WTP}_{\mathrm{i} 2}=\mathrm{WTP}_{\mathrm{i} 0}+\delta \mathrm{I}_{2}+\eta_{\mathrm{i} 2}$, where $\mathrm{E}\left(\mathrm{b}_{\mathrm{i} 1}\right)=\mathrm{E}\left(\mathrm{WTP}_{\mathrm{i} 0}\right)=100$.

(a) Mean WTP, (b) Standard Deviation of WTP 35

II.3. Simulation results for $\mathrm{WTP}_{\mathrm{i} 2}=\mathrm{WTP}_{\mathrm{i} 0}+\delta \mathrm{I}_{2}+\eta_{\mathrm{i} 2}$, where $\mathrm{E}\left(\mathrm{b}_{\mathrm{i} 1}\right)=50<$

$\mathrm{E}\left(\mathrm{WTP}_{\mathrm{i} 0}\right)=100$ (a) Mean WTP, (b) Standard Deviation of WTP 36

II.4. Simulation results for $\mathrm{WTP}_{\mathrm{i} 2}=\mathrm{WTP}_{\mathrm{i} 0}+\delta^{0} \mathrm{I}_{2}+\delta^{1} \mathrm{I}_{2}\left(\mathrm{~b}_{\mathrm{i} 2}\right)+\eta_{\mathrm{i} 2}, \mathrm{E}\left(\mathrm{b}_{\mathrm{i} 1}\right)=$ $\mathrm{E}\left(\mathrm{WTP}_{\mathrm{i} 0}\right)=100$. (a) Mean WTP, (b) Standard Deviation of WTP

II.5. Simulation results for $\mathrm{WTP}_{\mathrm{i} 2}=\mathrm{WTP}_{\mathrm{i} 0}+\delta^{0} \mathrm{I}_{2}+\delta^{1} \mathrm{I}_{2}\left(\mathrm{~b}_{\mathrm{i} 2}\right)+\eta_{\mathrm{i} 2}$, where $\mathrm{E}(\mathrm{bi1})=50<$ $\mathrm{E}(\mathrm{WTPi})=100$. (a) Mean WTP, (b) Standard Deviation of WTP 38

II.6. Mean WTP Simulation results for $\mathrm{WTP}_{\mathrm{i} 2}=\mathrm{WTP}_{\mathrm{i} 0}+\delta \mathrm{I}_{2}+\eta_{\mathrm{i} 2}$, and $\alpha_{\mathrm{ij}}=-20$

(a) $E\left(b_{i 1}\right)=E\left(W T P_{i 0}\right)=100$, (b) $E\left(b_{i 1}\right)=50<E\left(W T P_{i 0}\right)=100$

II.7. Mean WTP Simulation results for $\mathrm{WTP}_{\mathrm{i} 2}=\mathrm{WTP}_{\mathrm{i} 0}+\delta^{0} \mathrm{I}_{2}+\delta^{1} \mathrm{I}_{2}\left(\mathrm{~b}_{\mathrm{i} 2}\right)+\eta_{\mathrm{i} 2}$, and $\alpha_{\mathrm{ij}}=-20$ (a) $E\left(b_{i 1}\right)=E\left(W T P_{i 0}\right)=100$, (b) $E\left(b_{i 1}\right)=50<E\left(W T P_{i 0}\right)=100$

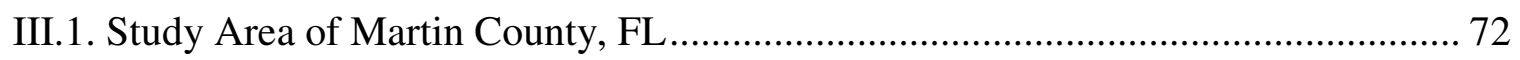

III.2. Martin County Waterfront Transactions January 2000 to August 2004 ................. 73

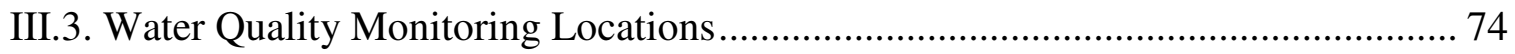

III.4. Example of Martin County Available Weekly Water Quality Data ...................... 75

III.5. DO Levels by WQ Monitoring Location from Jan 2000 to Dec 2004 .................... 76

III.6. Waterfront Home Sales by WQ Monitoring Location........................................ 77

III.7. a) Median Water Visibility by Year \& Location; b) Min, Avg, \& Max Water

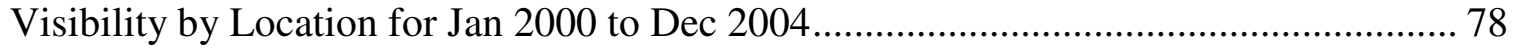

IV.1. Hurricane Dennis Forecast Advisory \#19...................................................... 85

IV.2. NHC Official Annual Average Track Errors, Atlantic Basin Storms .................... 86 
IV.3. NHC Official Annual Avg Intensity Errors Atlantic Basin Tropical Cyclones ...... 87

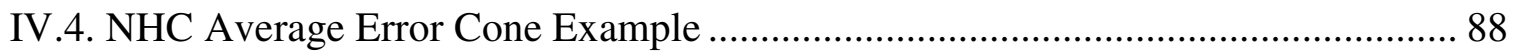

IV.5. HURREVAC Strike Probabilities for Hurricane Dennis Advisory \#19................. 91

IV.6. Hurricane Opal Cumulative Evacuation Response Curve................................... 93

IV.7. Fast, Medium, \& Slow S-Shaped Evacuation Response Curves ........................... 94

IV.8. Hurricane Jeanne Evacuation Response Curve by Household Location................ 95

IV.9. Hurricane Evacuation Response Rate by Household Type ................................ 95

IV.10. A Static Perspective of Period 1 Evacuation .................................................. 105

IV.11. Dynamic vs. Static Perspective of Period 1 Evacuation ................................... 106

IV.12. A Static Perspective of Period 1 Evacuation Given Structural Mitigation.......... 108

IV.13. A Dynamic Perspective of Period 1 Evac Given Structural Mitigation .............. 108

IV.14. A Static Perspective of Period 1 Evac Given Higher Prob of CAT 3 ............... 110

IV.15. A Dynamic Perspective of Period 1 Evac Given Higher Prob of CAT 3........... 111

IV.16. Dynamic View of Period 1 Evac Given Mitigation Focus on Minor

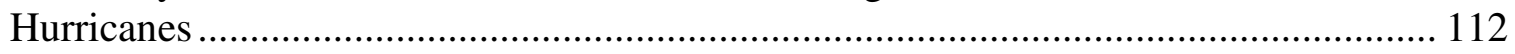

IV.17. A Dynamic Perspective of Period 1 Evacuation Given Evacuation Rebate........ 112

IV.18. Optimal Evacuation by Evacuation Cost Type............................................ 114

IV.19. Optimal Evac by Evacuation Cost Type Given $\$ 50$ Evacuation Cost

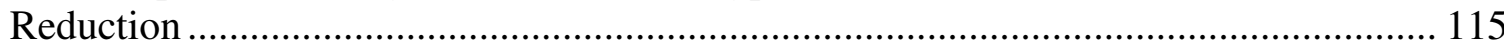

IV.20. Optimal Waiting when Evacuation Costs Increase by 10\% vs. 25\% …............. 117

IV.21. Unique Solution to Optimal Stopping Problem with Constant Costs of Evac .... 122

IV.22. 19 Identified Gulf of Mexico Historical Storm Tracks .................................. 126

IV.23. Example of the Determination of a Probability Of A Strike By CAT

Level Per Forecast Hour 
IV.24. Hurricane Dennis Landfall Locations.

IV.25. Cumulative Evacuation Timing Hurricane Bonnie 144

IV.26. Cumulative Evacuation Timing Hurricane Ivan 144

IV.27. Cumulative Evacuation Timing Hurricane Charley 145

IV.28. Cumulative Evacuation Timing Hurricane Frances 145

IV.29. Cumulative Evacuation Timing Hurricane Jeanne 146

IV.30. Cumulative Evacuation Timing Hurricane Lili 146

IV.31. Cumulative Evacuation Timing Hurricane Opal .... 147

IV.32. Cumulative Evacuation Timing Hurricane Floyd 147

IV.33. Derived CAT 1 to CAT 5 Costs of Evacuation 153

IV.34. Probability of Injury by SSHS CAT. 156

IV.35. Average Household Optimal Evacuation Results. 160

IV.36. High Damage Household Optimal Evacuation Results. 169

IV.37. Low Damage Household Optimal Evacuation Results 170

IV.38. High Overall Evacuation Cost Household Optimal Evacuation Results. 171

IV.39. Low Overall Evacuation Cost Household Optimal Evacuation Results 172

IV.40. Tourist Optimal Evacuation Results 173

IV.41. Effect of Lower Overall Costs of Evacuation on Evacuation Timing....... 174

IV.42. Optimal Evacuation Region Excluding Lost Income Costs ..... 178

IV.43. Optimal Evacuation Region Excluding Direct Costs 178

IV.44. Optimal Evacuation Region for Different Rates of Cost Increases 180

IV.45. High Damage Household Combined with Cost Reduction Optimal Evacuation Results 181 


\section{INTRODUCTION}

This dissertation comprises three essays, two of which are focused on the economic valuation of the environment, and another on economic decision making in the presence of an environmental disaster, i.e., hurricanes.

The implementation by researchers of a double-bound dichotomous choice (DBDC) contingent valuation methodology (CVM) over a single-bound dichotomous choice (SB-DC) CVM suggests incentive incompatible respondent behavior, which leads to biased (typically downward) willingness-to-pay (WTP) estimates (Carson, et al., 2000). Various specifications exist for researchers to attempt to identify respondent true WTP by accounting for this apparent shift of respondents' latent true WTP between responses to the first and second bid amounts, including models of structural shift (Alberini et al., 1997) and starting-point bias (Herriges and Shogren, 1996). This first essay develops a respondent model of Bayesian updating for a DB-DC CVM that is used to demonstrate how existing identifications of unbiased respondent WTP may often fail. However, we also show that a simple extension of the structural shift model, which is derived explicitly from our Bayesian updating behavioral model, can correct for much of the WTP bias.

While CVM respondents have been frequently modeled as Bayesian updaters (Horowitz, 1993; Herriges and Shogren, 1996; McLeod and Bergland, 1999; Whitehead, 2002; Flores and Strong, 2004; and Aadland et al., 2005), updating in a DB-DC CVM is typically restricted to the asking of the second bid amount ${ }^{1}$. If rational respondents are updating due to the second bid amount, we believe it is also reasonable to expect rational

\footnotetext{
${ }^{1}$ Aadland et al. (2005) is an exception to this in the DB-DC case, allowing updating on both the first and second bid amounts.
} 
respondents to be updating to the first bid amount, and we therefore develop a respondent model of Bayesian updating to allow for this. Consequently, our model of respondent Bayesian updating behavior may be interpreted as an extension of the traditional startingpoint bias models where respondents do not update prior to responding to the first bid amount.

Using our respondent model of Bayesian updating behavior, we derive structural shift specifications to allow for the identification of respondent true WTP in a DB-DC CVM given updating on the second bid amount only, as well updating on both bid amounts. These specifications are comparable to the traditional structural shift model of Alberini et al., (1997) which only includes a dummy variable for the asking of the second bid amount We show that even if respondent Bayesian updating is restricted only to occur with the asking of the second bid amount, the correct structural shift specification in this context includes an additional term that is a function of the second bid amount. When respondents Bayesian update on both bid amounts, we show that the correct structural shift specification in this context includes additional terms that are functions of the first and second bid amounts, and true WTP from the correctly specified structural shift model is not identifiable.

In order to demonstrate the extent of WTP bias in a respondent Bayesian updating context for the two identifiable structural shift models (the traditional model with only the dummy variable for the asking of the second bid amount, and the model we specify that also includes a term that is a function of the $2^{\text {nd }}$ bid amount), we simulate respondents updating on the second bid amount only, as well updating on both bid amounts. Our simulations show that the traditional structural shift estimation produces 
biased estimates of the true WTP when researcher and respondent prior beliefs of the true WTP are not congruent, a result that places a heavy emphasis on the precision of the survey pre-test and bid selection. Furthermore, this specification consistently produces biased estimates of the standard deviation of WTP. Conversely, our simulations show that the incorporation of the term that is a function of the $2^{\text {nd }}$ bid amount can correct for much of the WTP bias and standard deviation of WTP bias generated, except at high levels of respondent updating. Moreover, an empirical application of both of the identifiable structural shift models to the Alaska Exxon Valdez DB-DC dataset confirms the simulation outcomes, with the key result being that our simple extension of the traditional structural shift model is significantly different from zero.

Given the continued use of DB-DC CVMs by researchers and practitioners, as well as the persistent notion that respondents are in fact uncertain about their true WTP (see, e.g., Li and Mattsson, 1995; Ready et al, 1995; Cameron and Englin, 1997; Wang, 1997; Loomis and Ekstrand, 1998; Park, 2003), our results are noteworthy. Indeed, a practical solution is offered that identifies true WTP for uncertain respondents that are rationally acting as Bayesian updaters in a DB-DC CVM (certainly for those suspected of only updating on the second bid amount). The results also advise caution to the perception that respondents are acting strategically toward the asking of the second bid amount (Carson, et al., 2000), or as Aadland et al. (2005) state that, "Once one takes this Bayesian perspective of WTP formation, the recent discussion of the incentive incompatibility of DB-DC formats changes markedly."

Hedonic property value models are used to determine whether a relationship exists between an environmental amenity and housing prices, and if so, to impute implicit 
prices for the environmental amenity that can be used for welfare analysis. Although hedonic property value studies that utilize air or noise quality as the environmental amenity in the empirical analysis are more prevalent, a number of fairly recent studies that employ water quality (WQ) as the environmental amenity have been applied including Boyle et al. (1999), Leggett and Bockstael (2000), Michael et al. (2000), Poor et al. (2001), and Gibbs et al. (2002). The purpose of this second essay is to add to this WQ literature by capitalizing on a unique WQ dataset for a year-round, urban, and coastal housing market in Martin County, Florida. A major concern of this study is to assess the sensitivity of imputed valuations to the choice of WQ measure.

Often, hedonic property studies are limited to available environmental measures that may not be related to homeowners' perceptions (Michael et al. 2000). Additionally, there are questions as to whether homebuyers are incorporating a more scientific but less easily understood objective measure of water quality, or a less scientific but more easily understood subjective measure of WQ into their purchase decision (Epp and Al-Ani, 1979; Michael et al. 2000; Poor et al., 2001). The nature of our unique dataset allows us to effectively deal with these issues. Six WQ measures (temperature, $\mathrm{pH}$, water visibility, salinity, dissolved oxygen, and location grade) have been produced and documented weekly since 1998 for nine separate monitoring locations in our study area, and these measures are readily available to homeowners/buyers via the local newspaper and the internet. The sixth WQ measure, location grade, is a more easily understandable yet scientifically based objective measure of water quality with an A, B, C, D, or F letter grade and accompanying percentage $(90-100 \%, 80-90 \%, 70-80 \%, 60-70 \%$, and $<60 \%$ respectively) being assigned to each location depending upon the realized values of the 
five other objective WQ measures. In this way, results from the pure objective WQ measures are contrasted with the more easily understood yet objectively based WQ measure of location grade.

Boyle et al. (1999), Michael et al. (2000), Poor et al. (2001), and Gibbs et al. (2002) all demonstrate that the minimum water clarity value for the year of the home sale matters for Maine and New Hampshire lakefront homebuyers. However, these results are not easily transferable to an urban coastal housing market such as that of Martin County, FL. Here, not only are the water body and urban vs. rural settings different, but so is the timing of sales, which occur year-round and not just primarily in the summer months. Given a year-round housing market, a homebuyer focus on a minimum water clarity value for the year of the sale may not be the most appropriate as it plausibly could be for a mostly summer housing market in the Northeastern United States ${ }^{2}$. Therefore, in addition to employing minimum WQ values in the year of sale, we also incorporate other summary statistics that might matter in a year-round housing market such as the maximum, median, and statistical range.

In order to assess whether our various water quality measures affect waterfront housing prices in Martin County, FL we run pooled as well as time/location fixed effect regressions for our unbalanced panel dataset. Results indicate that our various forms of water quality do in fact matter to Martin County waterfront homebuyers. These results alone are especially relevant given the $\$ 1$ billion Indian River Lagoon South (IRLS) Everglades restoration effort being conducted to improve water quality in this area, and

\footnotetext{
${ }^{2}$ Leggett and Bockstael (2000) show that median fecal coliform for the year of the sale matters for a mixed urban and rural estuary/coastal setting in Maryland, but their data is also limited from April to September.
} 
the lack of any formal economic benefit analysis conducted to-date (USACE/SFWMD, 2002). However, similar to Michael et al. (2000) no consistent relationship by either any of the six particular water quality variables, or by any of the four particular descriptive statistics used is found. This further clearly indicates that more efforts need to be aimed at understanding what particular WQ variable and its statistical form homebuyers use in making their purchase decision if hedonic property models utilizing WQ measures are to be applied successfully.

The 2005 hurricane season was the most active hurricane season on record and left in its wake many poignant reminders concerning the critical role of evacuation, including the floodwater trapped citizens of New Orleans due to Hurricane Katrina, as well as the evacuation gridlock outside Houston caused by the impending Hurricane Rita. Projections of future hurricane seasons currently indicate continued high levels of hurricane activity, further emphasizing the need to better understand evacuation behavior in order to attempt to avoid similar evacuation breakdowns. Much of the evacuation research to date has focused on either the characteristics of those who evacuate, such as location/type of home, income level, etc., or the general difficulties associated with evacuating (Dash and Gladwin, 2005). In their overview of social science research needs related to hurricane forecasts and warnings, Gladwin et al. (2005, pg. 9) highlight the need for research that leads to "... modeling of evacuation behavioral response in more precise and comprehensive ways". Enhanced hurricane evacuation modeling in regard to the incorporation of decision makers' risk perceptions, beliefs, constraints, and abilities to decipher warning signals, as well as the dynamic nature of decision making in a non- 
linear communication environment are all repeated and accentuated themes resulting from the white papers associated with the Gladwin et al. (2005) report.

The purpose of this third essay is to respond to one of the hurricane evacuation issues by developing a dynamic model of hurricane evacuation behavior. Specifically, a household's evacuation decision is framed as an optimal stopping problem where every potential evacuation time period prior to the actual hurricane landfall, the household's optimal choice is either to evacuate, or to wait one more time period for a revised hurricane forecast. Given this theoretical framework, relevant policy questions that affect the timing of the household evacuation decision are analyzed.

In order to provide the intuition behind evacuation decision making in a dynamic framework, we present initial results from a simple, two-period model of evacuation. By utilizing the two-period model to contrast a dynamic vs. static "now or never" view of the optimal $1^{\text {st }}$ period evacuation decision, we show that the dynamic perspective results in $1^{\text {st }}$ period waiting being optimal for certain levels of evacuation costs, even thought $1^{\text {st }}$ period evacuation is optimal from a static perspective. We illustrate other distinct dynamic vs. static optimal waiting/evacuating results for a number of possible hurricanerelated policies such as a mitigation focus solely on potential damage from the more frequent, but less destructive minor hurricanes. Further benefits of the use of a dynamic framework to analyze evacuation behavior are highlighted with the simple, two-period model including an analysis of households with heterogeneous evacuation costs, and the ability of the two-period results to provide a deeper understanding of existing evacuation timing empirical outcomes. 
However, as we ultimately want to better explain actual evacuation behavior, we extend our hypothetical two-period model to a realistic multi-period setting that is calibrated using existing forecast and evacuation cost data for a specific region, coastal areas on the Gulf of Mexico. We show that the model does a good job of explaining actual evacuation behavior in specific hurricanes, as well as expected evacuation timing outcomes by various household types. From this calibrated dynamic framework, a number of policy questions that plausibly affect the timing of household evacuations are analyzed. For example, does building more shelters induce earlier evacuation? Additionally, the value of an improved hurricane forecast is an outcome of the policy analysis.

Whitehead (2003) estimates the probability of evacuation for varying levels of hurricane intensity, but does so from a static perspective as the timing of the probability of an evacuation for any particular storm intensity level is not addressed. However, the evacuation decision when faced by a hurricane threat has the three qualities of irreversibility, uncertainty, and the ability to wait for more information that characterize a decision process that is better understood from a dynamic modeling approach (Dixit and Pindyck, 1994). Standard empirical results from the evacuation literature such as the traditional S-shaped evacuation response curves (USACE, 2006a) indicate certain households wait while others evacuate, and therefore further underscore the need for a dynamic perspective of evacuation behavior. Likewise, only from a dynamic perspective can it be shown (as we do) that households may in fact be acting rationally by choosing to ignore evacuation warnings and waiting for more information, even though it could be the case that the benefits of evacuating (i.e., the expected avoided damage costs) 
outweigh the evacuation costs for a particular static snapshot of time - a situation that seemingly perplexes public evacuation authorities.

Moreover, modeling the evacuation decision process dynamically over many time periods with households having the ability to wait for more information, is analogous to a real-life evacuation decision situation where the National Hurricane Center (NHC) issues official forecast advisories every six hours once a tropical depression, tropical storm, or hurricane has developed. While Fu and Wilmot (2004) utilize a sequential choice model to estimate the probability of a household evacuating or waiting in each period of their dynamic multi-period framework, and further use their dynamic model results to provide clarification to the standard evacuation timing empirical outcomes, their research differs from ours in a number of significant ways. Importantly, we provide a theoretical model of dynamic evacuation behavior which is necessary for conducting policy analysis. Furthermore, our dynamic model is calibrated with forecast data from a number of storms across a number of locations which coincides directly with the six hour NHC forecast advisory timeline, and we explicitly address the costs of evacuation in a household's evacuation decision. Our research then serves as a contrast to the existing models of hurricane evacuation behavior by utilizing a theoretically-driven dynamic modeling approach that provides a more realistic interpretation to the multi-period evacuation decision process through the use of forecast and evacuation cost data, thereby helping to further bridge the knowledge gap between hurricane forecasts and evacuation timing behaviors. 


\section{WILLINGNESS-TO-PAY BIAS IN DICHOTOMOUS CHOICE SURVEYS}

\section{II.I. Introduction}

The implementation by researchers of a double-bound dichotomous choice (DBDC) contingent valuation methodology (CVM) over a single-bound dichotomous choice (SB-DC) CVM suggests incentive incompatible respondent behavior, which leads to biased (typically downward) willingness-to-pay (WTP) estimates (Carson, et al., 2000). Various specifications exist for researchers to attempt to identify respondent true WTP by accounting for this apparent shift of respondents' latent true WTP between responses to the first and second bid amounts, including models of structural shift (Alberini et al., 1997) and starting-point bias (Herriges and Shogren, 1996). This paper develops a respondent model of Bayesian updating for a DB-DC CVM that is used to demonstrate how existing identifications of unbiased respondent WTP may often fail. However, we also show that a simple extension of the structural shift model, which is derived explicitly from our Bayesian updating behavioral model, can correct for much of the WTP bias.

While CVM respondents have been frequently modeled as Bayesian updaters (Horowitz, 1993; Herriges and Shogren, 1996; McLeod and Bergland, 1999; Whitehead, 2002; Flores and Strong, 2004; and Aadland et al., 2005), updating in a DB-DC CVM is typically restricted to the asking of the second bid amount ${ }^{3}$. If rational respondents are updating due to the second bid amount, we believe it is also reasonable to expect rational respondents to be updating to the first bid amount, and we therefore develop a respondent model of Bayesian updating to allow for this. Consequently, our model of respondent

\footnotetext{
${ }^{3}$ Aadland et al. (2005) is an exception to this in the DB-DC case, allowing updating on both the first and second bid amounts.
} 
Bayesian updating behavior may be interpreted as an extension of the traditional startingpoint bias models where respondents do not update prior to responding to the first bid amount.

Using our respondent model of Bayesian updating behavior, we derive structural shift specifications to allow for the identification of respondent true WTP in a DB-DC CVM given updating on the second bid amount only, as well updating on both bid amounts. These specifications are comparable to the traditional structural shift model of Alberini et al., (1997) which only includes a dummy variable for the asking of the second bid amount We show that even if respondent Bayesian updating is restricted only to occur with the asking of the second bid amount, the correct structural shift specification in this context includes an additional term that is a function of the second bid amount. When respondents Bayesian update on both bid amounts, we show that the correct structural shift specification in this context includes additional terms that are functions of the first and second bid amounts, and true WTP from the correctly specified structural shift model is not identifiable.

In order to demonstrate the extent of WTP bias in a respondent Bayesian updating context for the two identifiable structural shift models (the traditional model with only the dummy variable for the asking of the second bid amount, and the model we specify that also includes a term that is a function of the $2^{\text {nd }}$ bid amount), we simulate respondents updating on the second bid amount only, as well updating on both bid amounts. Our simulations show that the traditional structural shift estimation produces biased estimates of the true WTP when researcher and respondent prior beliefs of the true WTP are not congruent, a result that places a heavy emphasis on the precision of the 
survey pre-test and bid selection. Furthermore, this specification consistently produces biased estimates of the standard deviation of WTP. Conversely, our simulations show that the incorporation of the term that is a function of the $2^{\text {nd }}$ bid amount can correct for much of the WTP bias and standard deviation of WTP bias generated, except at high levels of respondent updating. Moreover, an empirical application of both of the identifiable structural shift models to the Alaska Exxon Valdez DB-DC dataset confirms the simulation outcomes, with the key result being that our simple extension of the traditional structural shift model is significantly different from zero.

Given the continued use of DB-DC CVMs by researchers and practitioners, as well as the persistent notion that respondents are in fact uncertain about their true WTP (see, e.g., Li and Mattsson, 1995; Ready et al, 1995; Cameron and Englin, 1997; Wang, 1997; Loomis and Ekstrand, 1998; Park, 2003), our results are noteworthy. Indeed, a practical solution is offered that identifies true WTP for uncertain respondents that are rationally acting as Bayesian updaters in a DB-DC CVM (certainly for those suspected of only updating on the second bid amount). The results also advise caution to the perception that respondents are acting strategically toward the asking of the second bid amount (Carson, et al., 2000), or as Aadland et al. (2005) state that, "Once one takes this Bayesian perspective of WTP formation, the recent discussion of the incentive incompatibility of DB-DC formats changes markedly."

This paper is organized as follows: Section II outlines the respondent Bayesian updating model; Section III discusses the identification of true WTP given the Bayesian framework; Section IV provides an overview of the data simulation; Section V presents 
the results of the estimation; Section VI applies both of the identifiable structural shift models to the Alaska DB-DC dataset; and Section VII provides concluding comments.

\section{II.II. Respondent Bayesian Updating Model}

Each of the $i$ th individual DB-DC CVM respondents has $W T P_{i}$ consisting of two components

$$
W T P_{i}=\theta+\mu_{i}
$$

where $\theta$ is an unknown component that is common to all respondents, and $\mu_{i}$ is a known, idiosyncratic component. A possible interpretation of [1] is that respondent $i$ knows he values the natural resource that is the focus of the CVM by more or less than the average person, by an amount $\mu_{i}$. In this interpretation, the expectation over all individuals is simply $E(\mu)=0$. Although respondent $i$ does not know $\theta$, he holds prior beliefs that it is a draw from a normal distribution with mean $\bar{\theta}_{i}$ and variance $\sigma_{\theta}^{2}$.

Let $b_{i 1}$ and $b_{i 2}$ denote the first and second bid amounts offered to respondent $i$ as per the DB-DC CVM standard protocol. Given respondent $i$ 's WTP uncertainty, he interprets each of the $j=1,2$ offered bids as a signal of the true value of $\theta$ such that he believes

$$
b_{i j}=\left(\theta+\alpha_{i j}\right)+\varepsilon_{i j}
$$

where $\alpha_{i j}$ is a constant known by individual $i$, and he assumes that $\varepsilon_{i j} \sim N\left(0, \sigma_{\varepsilon_{i j}}{ }^{2}\right)$. That is, he interprets $b_{i j}-\alpha_{i j}$ as independent and unbiased signals of $\theta$. 
From [1], respondent $i$ 's prior belief of $W T P_{i}$ is that it is normally distributed with mean $\bar{\theta}_{i}+\mu_{i}$ and variance $\sigma_{\theta}^{2}$. Let $W T P_{i j}$ denote $E\left(W T P_{i}\right)$ after receiving $j$ offered bids. Then, $W T P_{i 0}=\bar{\theta}_{i}+\mu_{i}$. Using standard Bayesian formulae for normal conjugates ${ }^{4}, i^{\prime} \mathrm{s}$ posterior beliefs of $W T P_{i}$ after receiving the first bid, $b_{i l}$, is normal with mean

$$
W T P_{i 1}=\mu_{i}+\frac{\bar{\theta}_{i} \cdot \sigma_{\varepsilon_{i 1}}{ }^{2}}{\left(\sigma_{\theta}^{2}+\sigma_{\varepsilon_{i 1}}{ }^{2}\right)}+\frac{\left(b_{i 1}-\alpha_{i 1}\right) \cdot \sigma_{\theta}^{2}}{\left(\sigma_{\theta}^{2}+\sigma_{\varepsilon_{i 1}}{ }^{2}\right)}
$$

and variance

$$
\sigma_{i 1}^{2}=\frac{\sigma_{\theta}^{2} \cdot \sigma_{\varepsilon_{i 1}}^{2}}{\left(\sigma_{\theta}^{2}+\sigma_{\varepsilon_{i 1}}^{2}\right)}
$$

Given that the respondent is updating on both bid amounts under the reasonable assumption that they interpret both bids as being independent, when receiving the second bid, $b_{i 2}$, [3] and [4] become $i$ 's prior beliefs such that the posterior beliefs after hearing $b_{i 2}$ are also normal with mean

$$
W T P_{i 2}=\mu_{i}+\frac{\left(W T P_{i 1}-\mu_{i}\right) \cdot \sigma_{\varepsilon_{i 2}}{ }^{2}}{\left(\sigma_{i 1}{ }^{2}+\sigma_{\varepsilon_{i 2}}{ }^{2}\right)}+\frac{\left(b_{i 2}-\alpha_{i 2}\right) \cdot \sigma_{i 1}{ }^{2}}{\left(\sigma_{i 1}{ }^{2}+{\sigma_{\varepsilon_{i 2}}}^{2}\right)}
$$

and variance

$$
\sigma_{i 2}^{2}=\frac{\sigma_{i 1}^{2} \cdot \sigma_{\varepsilon_{i 2}}^{2}}{\left({\sigma_{i 1}}^{2}+\sigma_{\varepsilon_{i 2}}{ }^{2}\right)}
$$

Substituting for $\left(W T P_{i 1}-\mu_{i}\right)$ and ${\sigma_{i 1}}^{2}$ in [5] and [6] from [3] and [4], [5] and [6] can be rewritten such that

4 While other Bayesian updating representations could ostensibly be used, the normal conjugate importantly allows for tractable results 


$$
W T P_{i 2}=\mu_{i}+\frac{\left(b_{i 2}-\alpha_{i 2}\right)\left(\sigma_{\theta}{ }^{2} \cdot \sigma_{\varepsilon_{12}}{ }^{2}\right)}{\sigma_{\theta}{ }^{2}\left(\sigma_{\varepsilon_{11}}{ }^{2}+\sigma_{\varepsilon_{12}}{ }^{2}\right)+\left({\sigma_{\varepsilon_{12}}}^{2}\right)\left({\sigma_{\varepsilon_{11}}}^{2}\right)}+\frac{\left(\sigma_{\varepsilon_{i 2}}{ }^{2}\right)\left[\left(b_{i 1}-\alpha_{i 1}\right)\left(\sigma_{\theta}{ }^{2}\right)+\left(\bar{\theta}_{i}\right)\left(\sigma_{\varepsilon_{i 1}}{ }^{2}\right)\right]}{\sigma_{\theta}{ }^{2}\left({\sigma_{\varepsilon_{11}}}^{2}+{\sigma_{\varepsilon_{12}}}^{2}\right)+\left(\sigma_{\varepsilon_{12}}{ }^{2}\right)\left({\sigma_{\varepsilon_{11}}}^{2}\right)}
$$

and

$$
\sigma_{i 2}^{2}=\frac{\sigma_{\theta}^{2} \cdot \sigma_{\varepsilon_{i 1}}^{2} \cdot \sigma_{\varepsilon_{i 2}}^{2}}{\sigma_{\theta}^{2}\left(\sigma_{\varepsilon_{i 1}}^{2}+\sigma_{\varepsilon_{i 2}}{ }^{2}\right)+\left(\sigma_{\varepsilon_{i 2}}{ }^{2}\right)\left({\sigma_{\varepsilon_{i 1}}}^{2}\right)}
$$

Using $W T P_{i 0}=\bar{\theta}_{i}+\mu_{i}$, [3] and [7] can be simplified further to

$$
W T P_{i 1}=W T P_{i 0}+\frac{\left(b_{i 1}-\alpha_{i 1}-\bar{\theta}_{i}\right) \cdot \sigma_{\theta}^{2}}{\left(\sigma_{\theta}^{2}+\sigma_{\varepsilon_{i 1}}{ }^{2}\right)}
$$

and

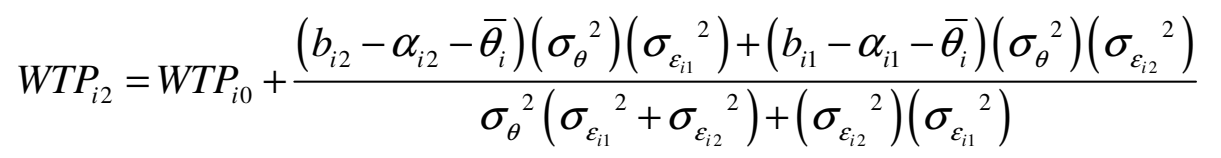

\section{II.III. Identification of True WTP}

In conducting a CVM, the goal of the researcher is to obtain the respondent's prior beliefs of WTP, $W T P_{i 0}$. For example, as Herriges and Shogren (1996, pg. 117) note, “... it is the household's prior held beliefs that the policymaker should be interested in, not the posterior WTP estimates that are artificially influenced by an optimal bid design." Therefore, we consider the ability to identify a respondent's true WTP, $W T P_{i 0}$, from our Bayesian updating framework for the three different possible respondent

signaling perspectives of our model: 1) neither bid provides a signal; 2) only the $2^{\text {nd }}$ bid provides a signal; or 3) both bids provide a signal. 


\section{Neither Bid Provides A Signal}

If respondent $i$ believes that neither of the $j=1,2$ offered bids contains a signal, then $\sigma_{\varepsilon_{i j}}{ }^{2} \rightarrow \infty$. If this is the case, then from [9] and [10], $W T P_{i 2}=W T P_{i 1}=W T P_{i 0}$. Therefore, true WTP can be identified from the responses to both questions by DB-DC estimation with associated efficiency gains over estimation using only responses to the first bid amount (Hanemann et al., 1991).

\section{2nd Bid Only Provides A Signal}

If it is the case that respondent $i$ believes that information concerning $\theta$ is contained in the second bid only, then ${\sigma_{\varepsilon_{i 1}}}{ }^{2} \rightarrow \infty$ and ${\sigma_{\varepsilon_{i 2}}}{ }^{2}<\infty$. From [9] we see that $W T P_{i 1}=W T P_{i 0}$. However, in this case $W T P_{i 2}$ does not follow from [10], as [3] and [4] no longer represent respondent $i$ 's prior beliefs when they receive $b_{i 2}$. Instead, respondent $i$

has prior beliefs with mean $\bar{\theta}_{i}$ and variance $\sigma_{\theta}^{2}$ when they receive $b_{i 2}$. Thus, again using standard Bayesian formulae for normal conjugates, $i$ 's posterior beliefs of $W T P_{i}$ after receiving $b_{i 2}$ is normal with mean

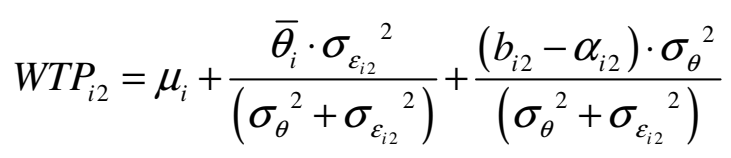

which, again using $W T P_{i 0}=\bar{\theta}_{i}+\mu_{i}$, can be simplified further to

$$
W T P_{i 2}=W T P_{i 0}+\frac{\left(b_{i 2}-\alpha_{i 2}-\bar{\theta}_{i}\right) \cdot \sigma_{\theta}^{2}}{\left(\sigma_{\theta}^{2}+\sigma_{\varepsilon_{i 2}}^{2}\right)}
$$

In this case, it therefore follows from [9] and [12] that $W T P_{i 2} \neq W T P_{i 1}=W T P_{i 0}$. Consequently, WTP estimates derived from the responses to the first bid are able to 
provide a consistent estimation of true WTP, but estimates derived from responses to both bids will be inconsistent unless an adequate control for the second response is introduced.

Alberini et al.'s (1997) structural shift dummy variable, adapted to our notation, is specified as

$$
\begin{aligned}
& W T P_{i 1}=W T P_{i 0}+\eta_{i} \\
& W T P_{i 2}=W T P_{i 0}+\delta_{i}+\eta_{i}
\end{aligned}
$$

where $\delta_{i}$ is the coefficient on a structural shift dummy variable that takes on the value one for responses to the second question. However, it is clear from [12] that the correct specification in a Bayesian updating context should also include an interaction term between $\delta_{i}$ and the magnitude of $b_{i 2}{ }^{5}$, that is

$$
\begin{aligned}
& W T P_{i 1}=W T P_{i 0}+\eta_{i} \\
& W T P_{i 2}=W T P_{i 0}-\left(\alpha_{i 2}+\bar{\theta}_{i}\right) \delta_{i}^{\prime}+\delta_{i}^{\prime}\left(b_{i 2}\right)+\eta_{i}
\end{aligned}
$$

where $\delta_{i}^{\prime}=\sigma_{\theta}{ }^{2} /\left(\sigma_{\theta}{ }^{2}+\sigma_{\varepsilon_{i 2}}{ }^{2}\right)$. Because $\alpha_{i 2}, \bar{\theta}_{i}, \sigma_{\theta}{ }^{2}$ and $\sigma_{\varepsilon_{i 2}}{ }^{2}$ are not observable, $-\left(\alpha_{i 2}+\bar{\theta}_{i}\right) \delta_{i}^{\prime}$ and $\delta_{i}^{\prime}$ are two individual-specific parameters. Assuming they are common to all individuals (Alberini et al., 1997) such that $\delta_{i}^{\prime}=\delta$, yields the system

$$
\begin{aligned}
& W T P_{i 1}=W T P_{i 0}+\eta_{i} \\
& W T P_{i 2}=W T P_{i 0}+\delta^{0} I_{2}+\delta^{1} I_{2}\left(b_{i 2}\right)+\eta_{i}
\end{aligned}
$$

where $I_{2}$ is a dummy variable indicating the asking of the second bid amount. From [15] we see that in a respondent Bayesian updating context, the correct structural shift specification is dependent upon the size of the second bid amount. Therefore, true WTP

\footnotetext{
5 Alberini et al. (1997, pg. 319) note that " $\delta$ could also be a function of additional explanatory variables including the cost amount or the change in cost amounts."
} 
is able to be identified from the responses to both questions with the appropriate dummy variable specification by stacking the data and estimating a conventional single-bound model (SB-DC) that has two observations for each respondent.

\section{Both Bids Provide A Signal}

Finally, if it is the case that respondent $i$ believes that information concerning $\theta$ is contained in both bid amounts, then ${\sigma_{\varepsilon_{i 1}}}^{2}<\infty$ and ${\sigma_{\varepsilon_{i 2}}}^{2}<\infty$. If this is the case, from [9] and [10] we have $W T P_{i 2} \neq W T P_{i 1} \neq W T P_{i 0}$. Consequently, unbiased estimates of WTP will only be able to be derived if an adequate control for both responses is implemented in the estimation.

Again, adapting Alberini et al.'s (1997) structural shift dummy variable to our notation with respondent updating on both bid amounts we have that

$$
\begin{aligned}
& W T P_{i 1}=W T P_{i 0}+\delta_{i 1}+\eta_{i} \\
& W T P_{i 2}=W T P_{i 0}+\delta_{i 2}+\eta_{i}
\end{aligned}
$$

where $\delta_{i l}$ is a coefficient on a structural shift dummy variable that takes on the value one for responses to the first question, and $\delta_{i 2}$ is a coefficient on a structural shift dummy variable that takes on the value one for responses to the second question. Allowing the $\delta_{i}$ 's to be functions of the bid amounts (which naturally follows from our respondent Bayesian updating context as per the second term on the right-hand side of both [9] and [10]) [16] can now be specified as

$$
\begin{aligned}
& W T P_{i 1}=W T P_{i 0}-\left(\alpha_{i 1}+\bar{\theta}_{i}\right) \delta_{i 1}^{\prime}+\delta_{i 1}^{\prime}\left(b_{i 1}\right)+\eta_{i} \\
& W T P_{i 2}=W T P_{i 0}-\left(\alpha_{i 2}+\bar{\theta}_{i}\right) \delta_{i 2}^{\prime}+\delta_{i 2}^{\prime}\left(b_{i 2}\right)-\left(\alpha_{i 1}+\bar{\theta}_{i}\right) \delta_{i 2}^{\prime \prime}+\delta_{i 2}^{\prime \prime}\left(b_{i 1}\right)+\eta_{i}
\end{aligned}
$$


where $\delta_{i 1}^{\prime}=\sigma_{\theta}{ }^{2} /\left(\sigma_{\theta}{ }^{2}+\sigma_{\varepsilon_{i 1}}{ }^{2}\right), \quad \delta_{i 2}^{\prime}=\left({\sigma_{\theta}}^{2}\right)\left({\sigma_{\varepsilon_{i 1}}}^{2}\right) /{\sigma_{\theta}}^{2}\left({\sigma_{\varepsilon_{i 1}}}^{2}+{\sigma_{\varepsilon_{i 2}}}^{2}\right)+\left({\sigma_{\varepsilon_{i 2}}}^{2}\right)\left({\sigma_{\varepsilon_{i 1}}}^{2}\right), \quad$ and $\delta_{i 2}^{\prime \prime}=\left(\sigma_{\theta}^{2}\right)\left({\sigma_{\varepsilon_{i 2}}}^{2}\right) / \sigma_{\theta}{ }^{2}\left({\sigma_{\varepsilon_{i 1}}}^{2}+\sigma_{\varepsilon_{i 2}}{ }^{2}\right)+\left({\sigma_{\varepsilon_{i 2}}}^{2}\right)\left({\sigma_{\varepsilon_{i 1}}}^{2}\right)$. Assuming the individual-specific parameters are common to all individuals, the following system is specified

$$
\begin{aligned}
& W T P_{i 1}=W T P_{i 0}+\delta^{0} I_{1}+\delta^{1} I_{1}\left(b_{i 1}\right) \eta_{i} \\
& W T P_{i 2}=W T P_{i 0}+\delta^{2} I_{2}+\delta^{3} I_{2}\left(b_{i 2}\right)+\delta^{4} I_{2}+\delta^{5} I_{2}\left(b_{i 1}\right)+\eta_{i}
\end{aligned}
$$

There are restrictions on these parameters, for example, if $\alpha_{i 1}=\alpha_{i 2}$, then $\delta^{2} / \delta^{3}=\delta^{4} / \delta^{5}$. But despite these potential restrictions, it is clear that $W T P_{i 0}$ cannot be identified.

For the three different possible respondent signaling perspectives of our Bayesian updating model, we have shown that the identification of true WTP is only possible for two of them given that the appropriate WTP estimation model has been specified. Since in conducting a CVM it is the goal of the researcher to obtain the respondent's true WTP, it is essential to understand the extent of bias (and if possible to correct for it) inherent in the estimated WTP if it is the case that respondents are updating on both bids and the researcher cannot specify the correct WTP estimation model, or where respondents are only updating on the second bid but the researcher has specified a WTP estimation model that does not contain the appropriate dummy variable specification.

\section{II.IV. Data Simulation}

In order to demonstrate the extent of WTP bias in a respondent Bayesian updating context for the two identifiable structural shift models (the traditional model, [13], and our extension of this model, [15]), we simulate respondents updating on the second bid 
amount only, as well updating on both bid amounts. Faced with a randomly selected bid amount, a CVM respondent will say yes to $b_{i j}$ when $W T P_{i j}$ is greater than $b_{i j}$, and no when it is less. Therefore, in a DB-DC CVM when respondents are updating on $b_{i 2}$ only, yes/no responses are generated according to:

$$
\text { yes }_{i 1}=\left\{\begin{array}{ll}
1 & W T P_{i 1}=W T P_{i 0}>b_{i 1} \\
0 & W T P_{i 1}=W T P_{i 0}<b_{i 1}
\end{array}, \quad y e s_{i 2}=\left\{\begin{array}{l}
1 \quad W T P_{i 2}=W T P_{i 0}+\frac{\left(b_{i 2}-\alpha_{i 2}-\bar{\theta}_{i}\right) \cdot \sigma_{\theta}{ }^{2}}{\left(\sigma_{\theta}{ }^{2}+\sigma_{\varepsilon_{i 2}}{ }^{2}\right)}>b_{i 2} \\
0 \\
W T P_{i 2}=W T P_{i 0}+\frac{\left(b_{i 2}-\alpha_{i 2}-\bar{\theta}_{i}\right) \cdot \sigma_{\theta}{ }^{2}}{\left(\sigma_{\theta}{ }^{2}+\sigma_{\varepsilon_{i 2}}{ }^{2}\right)}<b_{i 2}
\end{array}\right.\right.
$$

where $W T P_{i 0}=\bar{\theta}_{i}+\mu_{i}$. And, when respondents are updating on both $b_{i 1}$ and $b_{i 2}$, yes/no responses are generated according to:

$$
\begin{gathered}
\text { yes }_{i 1}= \begin{cases}1 & W T P_{i 1}=W T P_{i 0}+\frac{\left(b_{i 1}-\alpha_{i 1} \bar{\theta}_{i}\right) \cdot \sigma_{\theta}{ }^{2}}{\left(\sigma_{\theta}{ }^{2}+\sigma_{\varepsilon_{i 1}}{ }^{2}\right)}>b_{i 1} \\
0 & W T P_{i 1}=W T P_{i 0}+\frac{\left(b_{i 1}-\alpha_{i 1}-\bar{\theta}_{i}\right) \cdot \sigma_{\theta}{ }^{2}}{\left(\sigma_{\theta}{ }^{2}+\sigma_{\varepsilon_{i 1}}{ }^{2}\right)}<b_{i 1}\end{cases} \\
\operatorname{yes}_{i 2}=\left\{\begin{array}{l}
1 \quad W T P_{i 2}=W T P_{i 0}+\frac{\left(b_{i 2}-\alpha_{i 2}-\bar{\theta}_{i}\right)\left(\sigma_{\theta}{ }^{2}\right)\left(\sigma_{\varepsilon_{i 1}}{ }^{2}\right)+\left(b_{i 1}-\alpha_{i 1}-\bar{\theta}_{i}\right)\left(\sigma_{\theta}{ }^{2}\right)\left(\sigma_{\varepsilon_{i 2}}{ }^{2}\right)}{\sigma_{\theta}{ }^{2}\left(\sigma_{\varepsilon_{i 1}}{ }^{2}+\sigma_{\varepsilon_{i 2}}{ }^{2}\right)+\left(\sigma_{\varepsilon_{i 2}}{ }^{2}\right)\left(\sigma_{\varepsilon_{i 1}}{ }^{2}\right)}>b_{i 2} \\
0 \quad W T P_{i 2}=W T P_{i 0}+\frac{\left(b_{i 2}-\alpha_{i 2}-\bar{\theta}_{i}\right)\left(\sigma_{\theta}{ }^{2}\right)\left(\sigma_{\varepsilon_{i 1}}{ }^{2}\right)+\left(b_{i 1}-\alpha_{i 1}-\bar{\theta}_{i}\right)\left(\sigma_{\theta}{ }^{2}\right)\left(\sigma_{\varepsilon_{i 2}}{ }^{2}\right)}{\sigma_{\theta}{ }^{2}\left(\sigma_{\varepsilon_{i 1}}{ }^{2}+\sigma_{\varepsilon_{i 2}}{ }^{2}\right)+\left(\sigma_{\varepsilon_{i 2}}{ }^{2}\right)\left(\sigma_{\varepsilon_{i 1}}{ }^{2}\right)}<b_{i 2}
\end{array}\right.
\end{gathered}
$$

where $W T P_{i 0}=\bar{\theta}_{i}+\mu_{i}$.

We specify our values for $\bar{\theta}_{i}, \mu_{i}, \sigma_{\theta}{ }^{2}, \alpha_{i j}, \sigma_{\varepsilon_{i j}}{ }^{2}$, and $b_{i j}$ as summarized in Table $1 .^{6}$ For each of the eight specified $\sigma_{\varepsilon_{i 1}}$ values, per each of the three specified $b_{i 1}$ mean values of Table 1, we generate 1000 samples each of sample size 1000. Given the generated sample data, yes/no responses follow from [19] and [20]. Figure 1 provides an example of generated DB-DC yes/no responses for an illustrative respondent that does not

\footnotetext{
${ }^{6}$ Typical CVM initial bids are centered around a single value with specified increments (e.g., 25, 50, 75, $100,125,150,200)$. We have not specified any such increments in drawing our initial bids from a normal distribution. We do not feel this comprises the analysis.
} 
Bayesian update on either bid, updates on the second bid only $\left(\sigma_{\varepsilon_{i 2}}{ }^{2}=10\right)$, and updates on both bids $\left(\sigma_{\varepsilon_{i 1}}{ }^{2}=10\right.$, and ${\sigma_{\varepsilon_{i 2}}}^{2}=10)$.

\section{II.V. Estimation and Results}

In addition to the generated DB-DC yes/no responses and associated bid amounts from the data simulation, an intercept (the only independent variable used in order to represent $W T P_{i}$ ) and the appropriate $b_{i 2}$ dummy variable(s) from [13] and [15] complete the datasets to be estimated. The introduction of the structural shift dummy variable(s) requires the data to be stacked, and therefore maximum likelihood estimation of WTP follows from the conventional SB-DC model of Cameron and James (1987), but with two observations for each respondent. We perform probit and logit maximum likelihood estimation for the 1000 samples for each specification. Because probit and logit simulations are qualitatively similar, only logit estimation results are presented below.

\section{Structural Shift Model with only the Dummy Variable for the Asking of the Second Bid}

Figures 2(a) and 2(b) illustrate the results from [13] for the estimated mean WTP and standard deviation of WTP respectively, when the initial bid value is drawn from a normal distribution that is centered on the true WTP of 100 , and the respondents are updating on both bid amounts. Estimates of the 97.5 and 2.5 percentiles of mean WTP and standard deviation of WTP are also illustrated in Figures 2(a) and 2(b) respectively as a measure of the variability of these estimates across the eight specified $\sigma_{\varepsilon_{i 1}}$ values ${ }^{7}$.

\footnotetext{
${ }^{7}$ Results for $\sigma_{\varepsilon_{i 1}}=1000$ are not shown but are approximate to the results for $\sigma_{\varepsilon_{i 1}}=100$.
} 
Furthermore, although the results presented are based upon the simulated responses to both bid amounts, for high levels of $\sigma_{\varepsilon_{11}}$ (denoted sig eps_1 in the figures) along the xaxis, the results can be interpreted as respondents updating only on the second bid amount. In this way, the figures simultaneously present the results for the estimated mean WTP, standard deviation of WTP, and the associated 97.5 and 2.5 percentiles in both of the respondent Bayesian updating contexts. ${ }^{8}$

While Figure 2(a) shows that the results of estimated mean WTP are unbiased ${ }^{9}$ vs. the true value of 100, Figure 2(b) indicates that the estimated standard deviation of WTP is biased upward vs. the true value of 20 for all levels of $\sigma_{\varepsilon_{i 1}}$. These general bias results hold whether the respondent is updating on either both bid amounts, or only the second bid amount. However, the upward bias of the standard deviation of WTP becomes larger as less updating is occurring on the first bid amount. Additionally, while the variability of the estimates of mean WTP remains relatively constant over the specified levels of $\sigma_{\varepsilon_{i 1}}$, the variability of the estimates of standard deviation of WTP increases with higher levels of $\sigma_{\varepsilon_{i 1}}$ (i.e., with less updating on the first bid amount). Therefore, in the case where researchers select initial bid amounts from a distribution that is centered on respondent's prior beliefs of true WTP $=100$, unbiased estimates of mean WTP with relatively constant variability are generated, although the standard deviation of the these estimates is biased upward with both the bias and the variability of the standard deviation estimates increasing as respondents update less on the first bid amount.

\footnotetext{
${ }^{8}$ This is true for all of the other estimation figures associated with this model, namely Figures 3(a) and 3(b)

${ }^{9}$ T-tests at the $1 \%$ level are used to confirm the presence of bias for all estimation results of mean WTP and standard deviation of WTP unless otherwise noted.
} 
But what about the case where researchers prior beliefs of true WTP do not match to those of respondents, a case that seems to be more likely to occur in the implementation of a CVM? Figures 3(a) and 3(b) illustrate the results from [13] for the estimated mean WTP and standard deviation of WTP respectively when the initial bid value is drawn from a normal distribution that is not centered on the true WTP, i.e., $50<$ 100 , and the respondents are updating on both bid amounts ${ }^{10}$. In this case, both the estimates of the mean WTP and the standard deviation of WTP are biased when respondents are updating on both bid amounts, and also when respondents are updating only on the second bid amount. From Figure 3(a) we see that for strong updating on both bid amounts (low levels of $\sigma_{\varepsilon_{i 1}}$ ), mean WTP is biased downward from true WTP $=100$ with little variability in the estimates. In fact, for complete updating on the first bid amount $\left(\sigma_{\varepsilon_{i 1}}=0\right)$, estimated WTP is the mean of the bid distribution $=50$. However, with less updating on the first bid amount, estimated mean WTP is biased upward from true WTP $=100$ and contains more variability in the estimates. Estimated standard deviation of WTP is again biased upward vs. the true value of 20 for all levels of $\sigma_{\varepsilon_{i 1}}$, but in this case the upward bias and variability of the standard deviation estimates are more constant over the specified levels of $\sigma_{\varepsilon_{i 1}}$.

To better understand the source of the bias, Table 2 illustrates the shifts in the percentages of Yes-Yes, Yes-No, No-Yes, and No-No responses between respondents not updating on either bid, and those updating on both bids when $\sigma_{\varepsilon_{i 1}}=2$ and $\sigma_{\varepsilon_{i 2}}=10$. When respondents do not update on either bid presented to them, and given that the presented

\footnotetext{
${ }^{10}$ The opposite mean WTP graph is produced when the initial bid value is drawn from a normal distribution with mean exceeding the true WTP, i.e., $150>100$
} 
initial bid value is drawn from a normal distribution that is not centered on the true WTP, i.e., $50<100$, more than $90 \%$ of the DB-DC responses fall into either the Yes-Yes or Yes-No vote categories as would be expected. Due to the high levels of Yes votes in this non-updating scenario, responses primarily fall into bounded intervals only above the initial bid amount $=50$, and estimated mean WTP is able to move to the true WTP $=100$. However, when respondents are updating (relatively strongly) on both bid amounts, there is a remarkable decrease in Yes-Yes votes and corresponding increase in No-Yes and NoNo votes. As can be inferred from the Bayesian updating example of Figure 1, for strong enough updating as well as relatively close true WTP and initial bid amounts, initial yes responses in a non-updating context are easily reversed. Therefore, responses no longer primarily fall into bounded intervals only above the initial bid amount $=50$, and estimated mean WTP is not able to approach true WTP $=100$.

These overall estimation results for the traditional structural shift model indicate that, in a respondent Bayesian updating context, this model fails to generate unbiased estimates of mean WTP unless the initial bid amount is centered on respondent's prior beliefs. Unfortunately, achieving initial bid amounts that are centered on respondent's prior beliefs is a case that would appear to be seemingly rare in practice, or at the very least places a heavy burden on the typical CVM pre-test. That is, it is reasonable to assume that pre-test respondents would also be Bayesian updating, and therefore results from a pre-test would not provide any further insight into how to adjust the bid amounts to be centered on respondent's prior beliefs of what is true WTP. Moreover, these overall estimation results for the traditional structural shift model indicate that, in a respondent 
Bayesian updating context, this model always fails to generate unbiased estimates of the standard deviation of WTP.

\section{Structural Shift Model that also Includes a Term that is a Function of the Second Bid}

Figures 4(a) and 4(b) illustrate the results from [15] for the estimated mean WTP and standard deviation of WTP respectively when the initial bid value is drawn from a normal distribution that is centered on the true WTP of 100 , and the respondents are updating on both bid amounts. Estimates of the 97.5 and 2.5 percentiles of mean WTP and standard deviation of WTP are also illustrated in Figures 4(a) and 4(b) respectively as a measure of the variability of these estimates across the eight specified $\sigma_{\varepsilon_{i 1}}$ values ${ }^{11}$.

Furthermore, although the results presented are based upon the simulated responses to both bid amounts, for high levels of $\sigma_{\varepsilon_{i 1}}$ (denoted sig eps_1 in the figures) along the xaxis, the results can be interpreted as respondents updating only on the second bid amount. In this way, the figures simultaneously present the results for the estimated mean WTP, standard deviation of WTP, and the associated 97.5 and 2.5 percentiles in both of the respondent Bayesian updating contexts. ${ }^{12}$

While Figure 4(a) shows that the results of estimated mean WTP are still unbiased vs. the true value of 100 , Figure 4(b) indicates that the previous bias in the standard deviation of WTP $=20$ from the traditional structural shift model of Figure 2(b) has dissipated. Furthermore, the variability of both the estimated mean WTP and standard deviation of WTP has decreased significantly as evidenced by the tighter 97.5 and 2.5

\footnotetext{
${ }^{11}$ Results for $\sigma_{\varepsilon_{i 1}}=1000$ are not shown, but are approximate to the results for $\sigma_{\varepsilon_{i 1}}=100$.

${ }^{12}$ This is true for all of the other estimation figures associated with this model, namely Figures 5(a) and
} $5(\mathrm{~b})$ 
percentile lines. However, we do start to see evidence of increased variability of mean WTP estimates, as well as evidence of bias and increased variability of estimates for the standard deviation of WTP for high levels of updating on bid 1 (low levels of $\left.\sigma_{\varepsilon_{i 1}}\right)^{13}$. These results at the least therefore indicate that this specification does a better job then the traditional structural shift model in producing unbiased estimates of the standard deviation of WTP when it is believed that respondents update only on the second bid amount.

Figures 5(a) and 5(b) illustrate results from [15] for the estimated mean WTP and standard deviation of WTP respectively when the initial bid value is drawn from a normal distribution that is not centered on the true WTP, i.e., $50<100$, and the respondents are updating on both bid amounts. Contrasting Figures 5(a) and 5(b) with Figures 3(a) and 3(b) we clearly see the improvement in reduced bias over the traditional structural shift model for both the estimates of mean WTP and the standard deviation of WTP. We also see improvements in the variability of both the estimated mean WTP and standard deviation of WTP as evidenced by the tighter 97.5 and 2.5 percentile lines. We again, however, start to see evidence of increased variability of mean WTP estimates, as well as evidence of bias and increased variability of estimates for the standard deviation of WTP for high levels of updating on bid 1 (low levels of $\left.\sigma_{\varepsilon_{i 1}}\right)^{14}$.

\footnotetext{
${ }^{13}$ Convergence issues at these low levels (i.e., $\sigma_{\varepsilon_{i 1}}<10$ ) prevent us at this time from making a more definitive statement concerning bias and depicting the results graphically.

${ }^{14}$ Convergence issues at these low levels (i.e., $\sigma_{\varepsilon_{i 1}}<10$ ) prevent us at this time from making a more definitive statement concerning bias and depicting the results graphically.
} 
These overall estimation results for the structural shift model we specify that also includes a term that is a function of the $2^{\text {nd }}$ bid amount indicate that in a respondent Bayesian updating context, unbiased and less variable estimates of mean WTP and standard deviation of WTP can be generated. The results certainly hold well for the case where respondents are only updating on the second bid amount as is typically perceived in the DB-DC CVM literature. For the case where respondents are updating on both bids, even though there is some indication of bias for high levels of updating on bid 1, obvious improvement over the traditional structural shift model in terms of reduced bias estimates of mean WTP and standard deviation of WTP is demonstrated.

\section{Investigating Respondent Strategic Behavior}

DB-DC WTP bias from a structural shift model is typically indicated as being downward due to the estimated negative $\delta$ coefficient (Alberini et al., 1997; Whitehead, 2002). Furthermore, Carson et al. (2000) have discussed various strategic behavior theories as to how agents may interpret this second price signal in order to explain the WTP downward bias. We show, in fact that it is the asymmetry induced by the standard DB-DC CVM protocol of halving $b_{i 1}$ for an initial no response, and doubling $b_{i l}$ for an initial yes response that generates the negative $\delta$ coefficient in a respondent Bayesian updating context, not necessarily respondent strategic behavior.

Table 3 presents results from two different estimations of [13] when the initial bid value is drawn from a normal distribution that is centered on the true WTP of 100, and the respondents are updating on both bid amounts with $\sigma_{\varepsilon_{i 1}}{ }^{2}=25$, and $\sigma_{\varepsilon_{i 2}}{ }^{2}=10$. In the first estimation, $b_{i 2}$ is generated by halving $b_{i 1}$ for an initial no response, and doubling 
$b_{i 1}$ for an initial yes response (the standard DB-DC CVM protocol). In the second estimation, $b_{i 2}$ is generated as $\left(b_{i 1}-60\right)$ for an initial no response, and $\left(b_{i 1}+60\right)$ for an initial yes response. We do generate a (-) $\delta$ coefficient in the standard halving/doubling $b_{i 2}$ generation, but the (-) $\delta$ coefficient disappears in our $\left[b_{i 1}(+) /(-) 60\right]$ estimation. Clearly, respondent strategic behavior cannot be inferred simply from the generation of a (-) $\delta$ coefficient for a DB-DC CVM where $b_{i 2}$ is generated by halving $b_{i 1}$ for an initial no response, and doubling $b_{i 1}$ for an initial yes response and respondents are acting as Bayesian updaters.

Our simulation results already presented in Figures 2(a) - 5(b) have all assumed the respondent's known constant of the signal, $\alpha_{i j}$, from [2] to equal 0 . If believing that respondents are in fact acting strategically similarly to one of the Carson et al. (2000) strategic behavior theories, allowing $\alpha_{i j} \neq 0$ allows for investigation of bias in this strategic behavior context. For example, if respondents feel that the researcher has placed them into a bargaining situation they will feel that the $b_{i j}$ presented to them has been purposefully inflated. In this case, $\alpha_{i j}<0$ in order to counteract the perceived bid inflation.

Figures $6(\mathrm{a})-7(\mathrm{~b})$ present mean WTP simulation results ${ }^{15}$ with $\alpha_{i j}=-20$ for both structural shift identifications of [13] and [15], as well as where $b_{i l}=$ true WTP $=100$ and where $b_{i l}=50<$ true WTP $=100$. In this strategic behavior context, we now see upward bias being generated for the case where researcher priors are compatible with respondent

\footnotetext{
${ }^{15}$ Standard deviation of WTP graphical results are not presented, but are still biased as was the case where $\alpha_{i j}=0$.
} 
priors of true WTP $=100$ as shown by Figure 6(a). The structural shift specification including the term for $b_{i 2}$ still appears to be able to correct for the generated bias as shown by Figure 7(a), although not at as low of levels of $\sigma_{\varepsilon_{i 1}}$ as when $\alpha_{i j}=0$. These results indicate that the specification of [15] is even more important in a possible strategic behavior Bayesian updating context.

\section{II.VI. Empirical Application}

Carson et al. (1992) conducted a DB-DC CVM for the State of Alaska in order to obtain a WTP value "to prevent another Exxon Valdez type oil spill". Median WTP = \$31 was estimated from respondents' answers to both CVM questions using an interval DB-DC model assuming a Weibull distribution. As a check on the sensitivity of the estimated DB-DC median WTP value, median WTP $=\$ 41$ was also estimated from answers to the first question only using a SB-DC model assuming a log-normal distribution. Given the disparity between the SB-DC and DB-DC WTP estimates, they conclude that a slight downward bias exists between respondents' answers to only the first bid amount and answers to both bid amounts. Indeed, Carson et al. (2003) note that the structural shift model of Alberini et al. (1997) could be used to account for this downward bias. We therefore use the Alaska dataset to estimate WTP from the two identifiable structural shift models of this paper (the traditional model, [13], and our extension of this model, [15]).

Table 4 presents the maximum likelihood estimates following from the conventional SB-DC model of Cameron and James (1987) using the Alaska study responses to the first bid amount only. Additionally, Table 4 presents the maximum 
likelihood estimates for the two identifiable structural shift models of [13] and [15] using the Alaska study responses to both bid amounts with the data being stacked to account for the introduction of the structural shift dummy variable(s). Only an intercept and the appropriate $b_{i 2}$ dummy variable(s) from [13] and [15] are used in the estimation, and a log-normal distribution has been assumed in order to follow the results of Carson et al. (1992).

Our SB-DC estimation produces an estimate of 3.73 for the intercept, equating to a median $\mathrm{WTP}^{16}=\$ 41.58$, with the standard deviation of WTP estimated at 3.15. This WTP result closely mirrors the median WTP $=\$ 41.44$ of Carson et al. (1992). The traditional structural shift model of [13] produces an estimate of 4.18 for the intercept, equating to median WTP $=\$ 65.54$, and 19.73 for the standard deviation of WTP. Also, although a negative coefficient is generated for the $2^{\text {nd }}$ question dummy variable, it is significant only up to the $10 \%$ level. The structural shift model of [15] that we specify that also includes a term that is a function of the $2^{\text {nd }}$ bid amount produces an estimate of 3.83 for the intercept, equating to median $\mathrm{WTP}=\$ 46.14$, and 7.01 for the standard deviation of WTP. Importantly, the additional term that is a function of the $2^{\text {nd }}$ bid amount is significant at the $1 \%$ level. A standard likelihood ratio test between [13] and [15] indicates that [15] in fact fits the Alaska data better.

This empirical application demonstrates, similar to our simulations, that if one believes respondents are only updating on the second bid amount and hence true WTP is represented by SB-DC estimates, than the structural shift model of [15] does a better job of estimating a less biased true WTP when utilizing a DB-DC CVM approach compared

\footnotetext{
${ }^{16}$ Given the log-normal distribution, median WTP $=\exp \left(\beta x^{\prime}\right)$ with $\beta x^{\prime}$ being the intercept.
} 
to the traditional structural shift model of [13]. Of course, if respondents are updating on both bid amounts, true WTP may not be identifiable as has been shown.

\section{II.VII. Conclusions}

We have shown why existing structural shift models used to estimate unbiased WTP from a DB-DC CVM are theoretically incapable of doing so in a Bayesian updating context due to misspecification and identification issues. Through our data simulations we have demonstrated the extent of the WTP bias when the identifiable, yet misspecified structural shift model is used. The results are most serious when researcher and respondent prior beliefs of true WTP are not congruent. We suggest a more properly specified structural shift model following from the respondent Bayesian updating behavioral model that includes an additional term that is a function of the second bid amount. Our data simulations show that this specification can correct for much of the potential WTP bias. An empirical application to the Alaska Exxon Valdez DB-DC dataset confirms the simulation outcomes, with the key result being that our simple extension of the traditional structural shift model is significantly different from zero.

The results of the paper also offer an alternative to the perception that respondents act strategically in a DB-DC CVM, and that their responses are not incentive compatible between questions. Rather, uncertain respondents act rationally by incorporating information signaled to them through both of the presented bid amounts. Not accounting for this possibility in the structural shift estimation leads to biased estimates of the respondent's true WTP. 
Table II.1. Specified Values for $\bar{\theta}_{i}, \mu_{i}, \sigma_{\theta}{ }^{2}, \alpha_{i j}, \sigma_{\varepsilon_{i j}}{ }^{2}$, and $b_{i j}$ Used In the Data Simulation

\begin{tabular}{|lcl|}
\hline Category & Variable & \multicolumn{1}{l}{ SpecifiedValue } \\
$\bar{\theta}_{i}$ & $100 \forall i$ \\
$\mu_{i 0}$ & $\mu_{i}$ & $\sim \mathrm{N}(0, \sigma)$ and $\sigma=20$ \\
$\begin{array}{l}\text { Standard Deviation } \\
\text { of Prior Beliefs }\end{array}$ & $\sigma_{\theta}$ & 20 \\
$\begin{array}{l}\text { Signal known } \\
\text { constant }\end{array}$ & $\alpha_{i j}$ & 0 \\
$\begin{array}{l}\text { Strength of } \\
\text { Updating }\end{array}$ & $\sigma_{\varepsilon_{i 1}}$ & $1000,100,50,25,10,5,2,0$ \\
Bids & $\sigma_{\varepsilon_{i 2}}$ & 10 \\
& $b_{i 1}$ & $\sim \mathrm{N}(100, \sigma), \sim \mathrm{N}(50, \sigma), \sim \mathrm{N}(150, \sigma)$ and $\sigma=30$ \\
& $b_{i 2}$ & $\left(b_{i 1}\right)^{*} 2$, or $\left(b_{i 1}\right) * 1 / 2$ for yes or no to $b_{i 1}$ respectively \\
\hline
\end{tabular}

Table II.2. $\%$ of YY, YN, NY, NN Responses for $W T P_{i 2}=W T P_{i 0}+\delta I_{2}+\eta_{i 2}$

\begin{tabular}{|c|c|c|}
\hline$\underline{\mathrm{DB}-\mathrm{DC}}$ & No & Updating on \\
\hline$\underline{\text { Response }}$ & Updating & $\underline{\text { Both Bids }}$ \\
\hline YY & $50 \%$ & $8 \%$ \\
\hline YN & $42 \%$ & $43 \%$ \\
\hline NY & $8 \%$ & $34 \%$ \\
\hline $\mathrm{NN}$ & $0 \%$ & $15 \%$ \\
\hline
\end{tabular}


Table II.3. $\delta$ Coefficient Results For Halving/Doubling, And (+)/(-) $60 b_{i 2}$ Generation

\begin{tabular}{|ccc|}
\hline & $\begin{array}{c}\text { Estimates } \\
\text { halving/doubling }\end{array}$ & $\frac{\text { Estimates }}{(+) /(-) 60}$ \\
\cline { 2 - 3 } WTP & 100.1 & 100.0 \\
$\delta$ & -18.9 & 0.2 \\
$\sigma$ & 93.7 & 135.3 \\
\hline
\end{tabular}

Table II.4: Alaska Study Maximum Likelihood Estimates

\begin{tabular}{|ccccc|}
\hline Parameter & $\underline{\text { SB-DC }}$ & & $\begin{array}{c}\text { Traditional } \\
\text { Structural Shift }\end{array}$ & $\begin{array}{c}\text { Structural Shift } \\
\text { with Bid Interaction }\end{array}$ \\
intercept & 3.7276 & 4.1827 & 3.8316 \\
& $(29.91)$ & $(5.064)$ & $(13.784)$ \\
$\delta^{0}$ & & -7.7975 & -4.1345 \\
& & $(-1.723)$ & $(-4.232)$ \\
$\delta^{l}$ & & & 0.0216 \\
& & & $(4.606)$ \\
$\sigma$ & 3.1493 & 19.7323 & 7.0067 \\
& $(7.293)$ & $(1.785)$ & $(3.886)$ \\
$\log L$ & & & \\
$\mathrm{n}$ & -695.52 & -1400.00 & -1392.07 \\
& 1043 & 2086 & 2086 \\
\hline
\end{tabular}

Note: t-statistics are in parenthesis 
Figure II.1. An Example of Generated Yes/No Responses Based Upon No Bayesian Updating, Updating on the Second Bid Only, and Updating on Both Bids for a Single Respondent

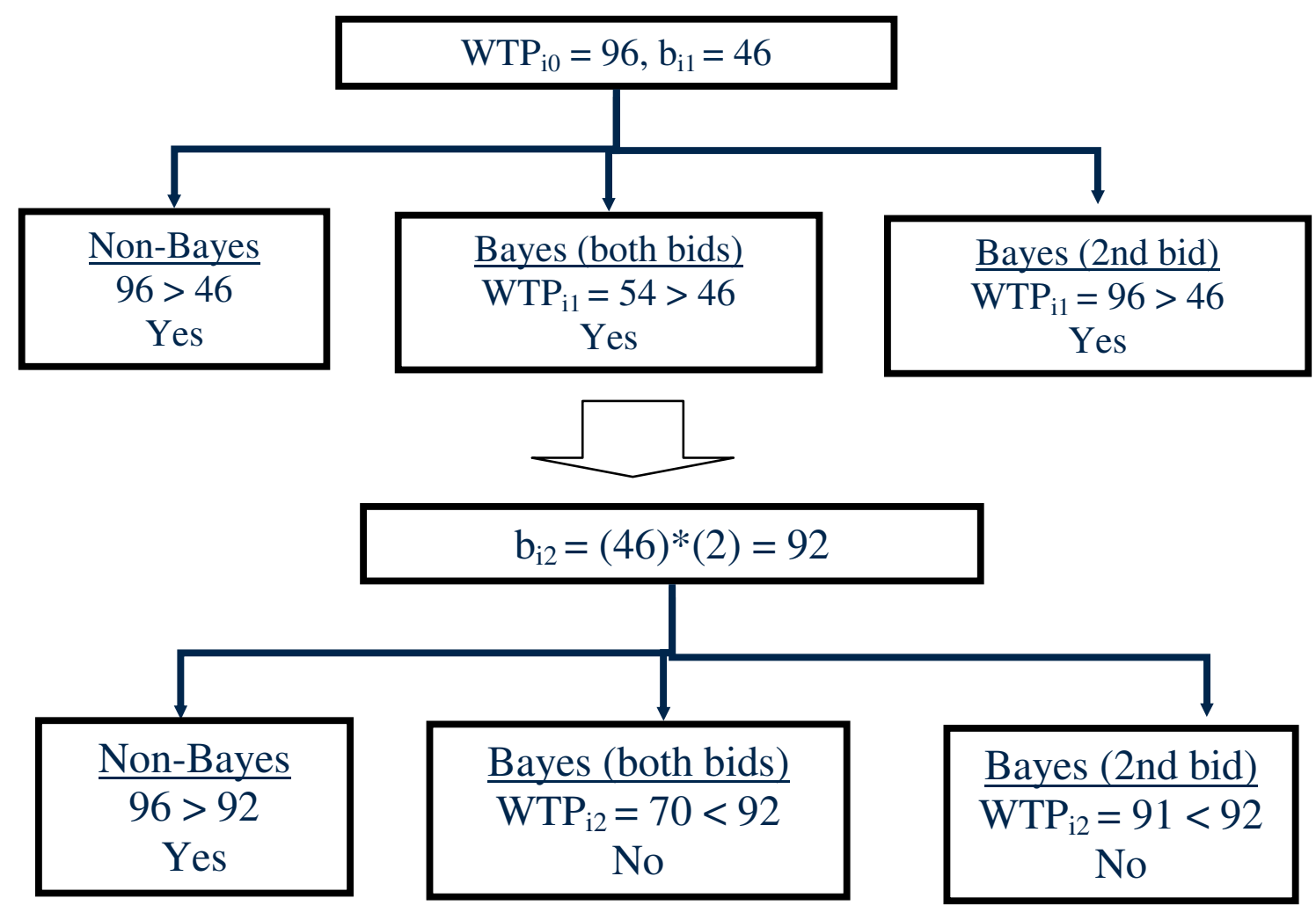

Yes/No Results:

Non-Bayes

Bayes (both)

Bayes (2nd

$\begin{array}{rr}\text { Bid 1 } & \text { Bid 2 } \\ 1 & 1 \\ 1 & 0 \\ 1 & 0\end{array}$


Figure II.2. Simulation results for $W T P_{i 2}=W T P_{i 0}+\delta I_{2}+\eta_{i 2}$, where $E\left(b_{i 1}\right)=E\left(W T P_{i 0}\right)=100$. (a) Mean WTP, (b) Standard Deviation of WTP
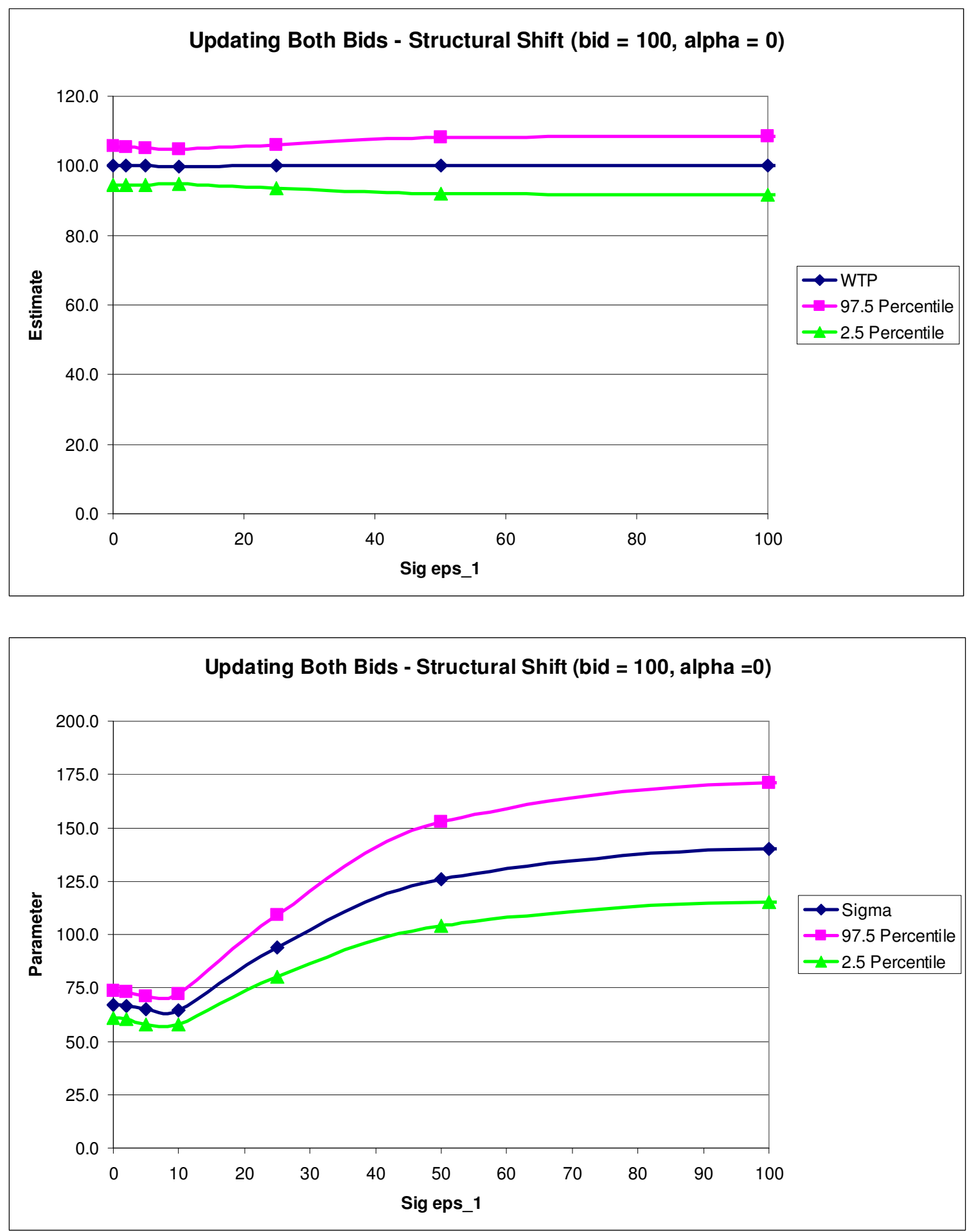
Figure II.3. Simulation results for $W T P_{i 2}=W T P_{i 0}+\delta I_{2}+\eta_{i 2}$, where $E\left(b_{i 1}\right)=50<$ $E\left(W T P_{i 0}\right)=100$ (a) Mean WTP, (b) Standard Deviation of WTP
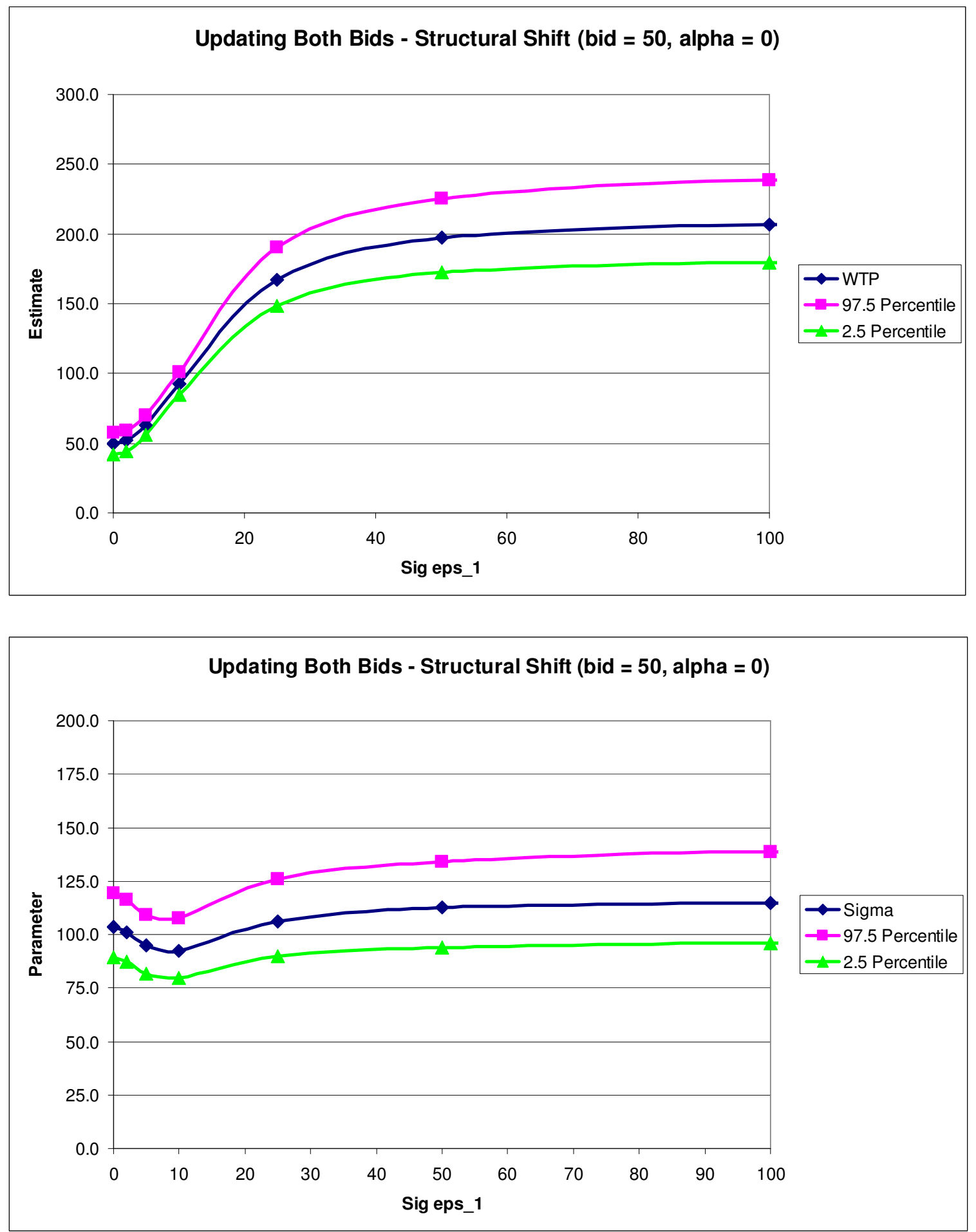
Figure II.4. Simulation results for $W T P_{i 2}=W T P_{i 0}+\delta^{0} I_{2}+\delta^{1} I_{2}\left(b_{i 2}\right)+\eta_{i 2}$, $E\left(b_{i 1}\right)=E\left(W T P_{i 0}\right)=100$. (a) Mean WTP, (b) Standard Deviation of WTP
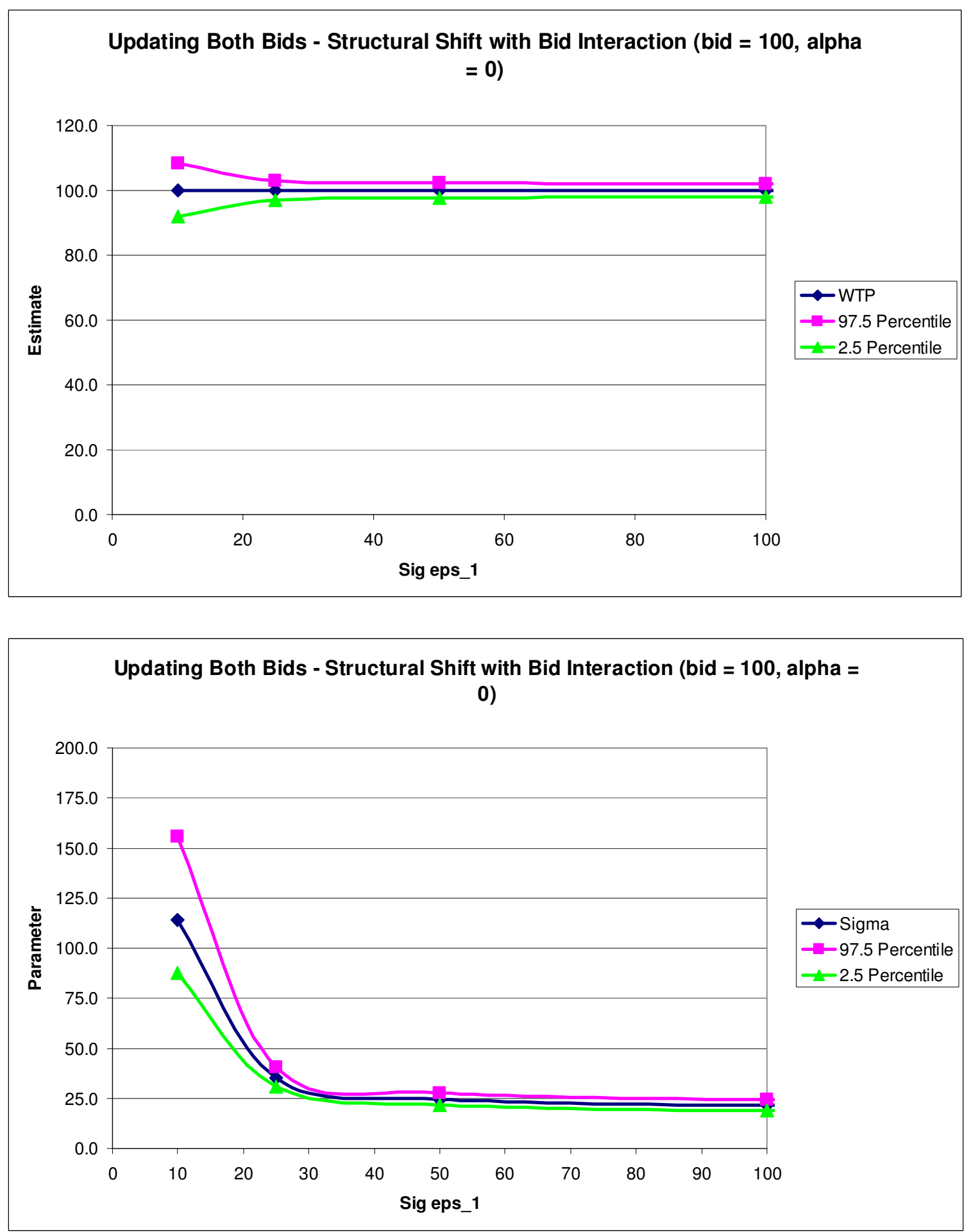
Figure II.5. Simulation results for $W T P_{i 2}=W T P_{i 0}+\delta^{0} I_{2}+\delta^{1} I_{2}\left(b_{i 2}\right)+\eta_{i 2}$, where $\mathrm{E}($ bi1 $)=50<\mathrm{E}(\mathrm{WTPi})=100$. (a) Mean WTP, (b) Standard Deviation of WTP
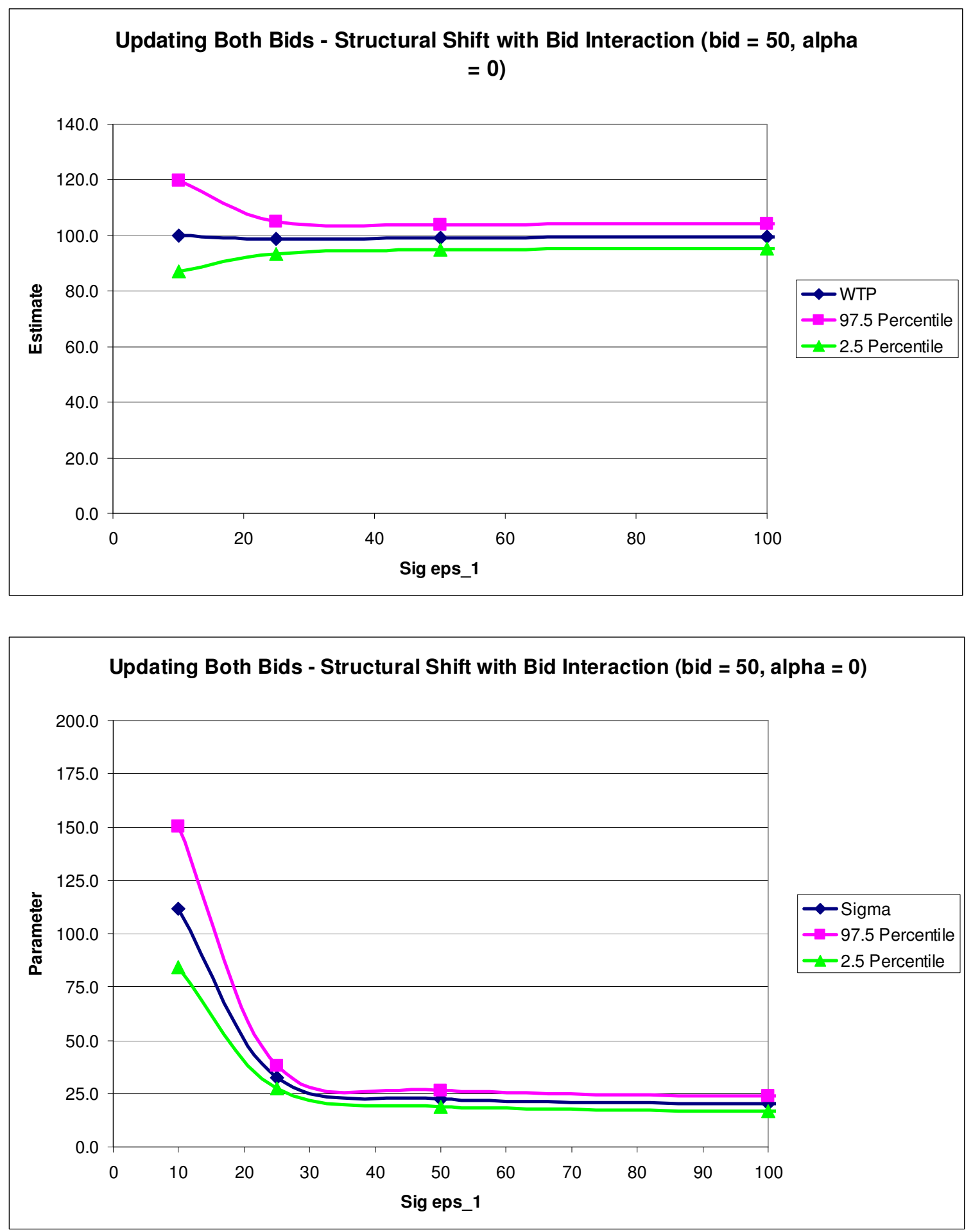
Figure II.6. Mean WTP Simulation results for $W T P_{i 2}=W T P_{i 0}+\delta I_{2}+\eta_{i 2}$, and $\alpha_{i j}=-20$. (a) $E\left(b_{i 1}\right)=E\left(W T P_{i 0}\right)=100$, (b) $E\left(b_{i 1}\right)=50<E\left(W T P_{i 0}\right)=100$
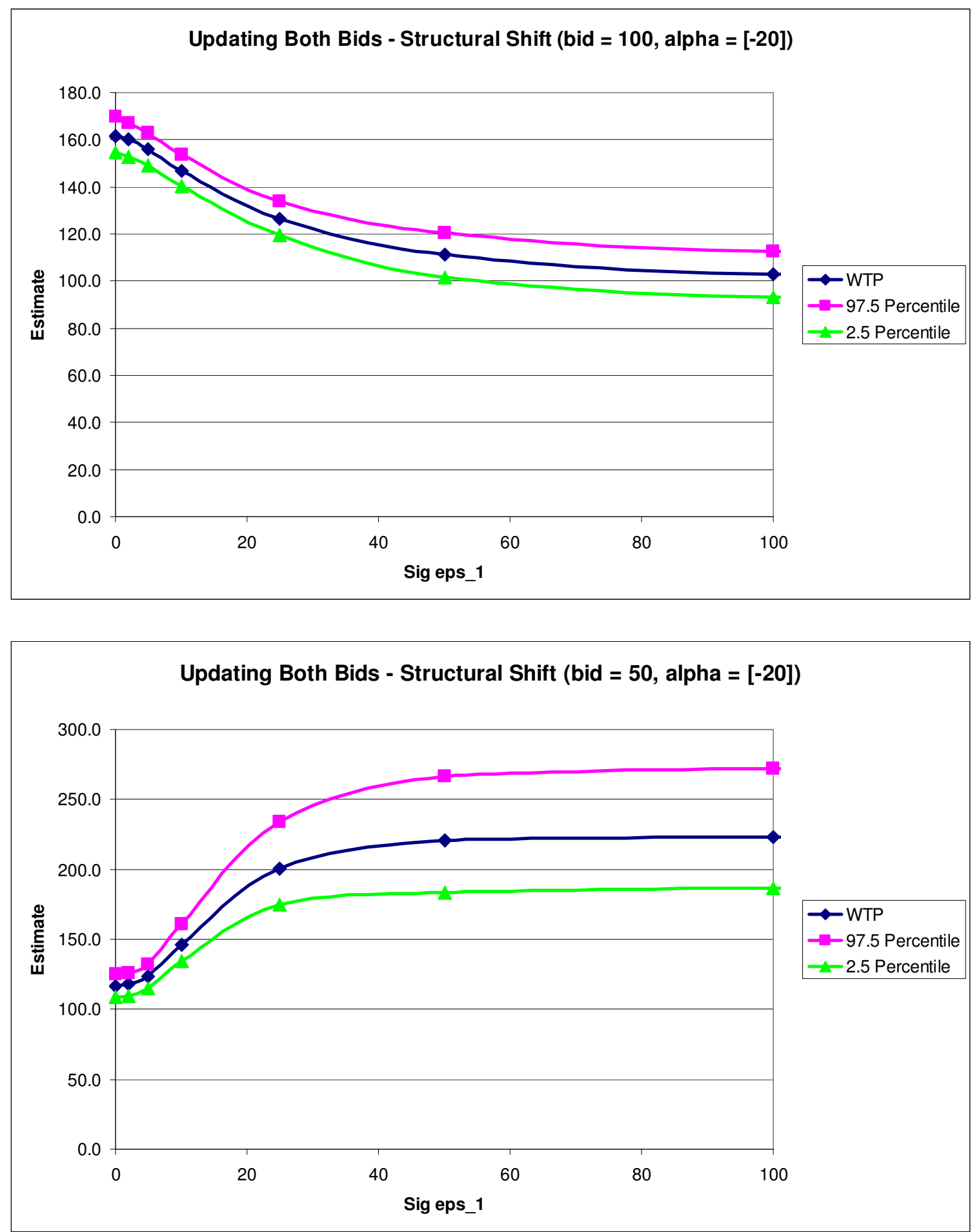
Figure II.7. Mean WTP Simulation results for $W T P_{i 2}=W T P_{i 0}+\delta^{0} I_{2}+\delta^{1} I_{2}\left(b_{i 2}\right)+\eta_{i 2}$, and $\alpha_{i j}=-20$ (a) $E\left(b_{i 1}\right)=E\left(W T P_{i 0}\right)=100$, (b) $E\left(b_{i 1}\right)=50<E\left(W T P_{i 0}\right)=100$
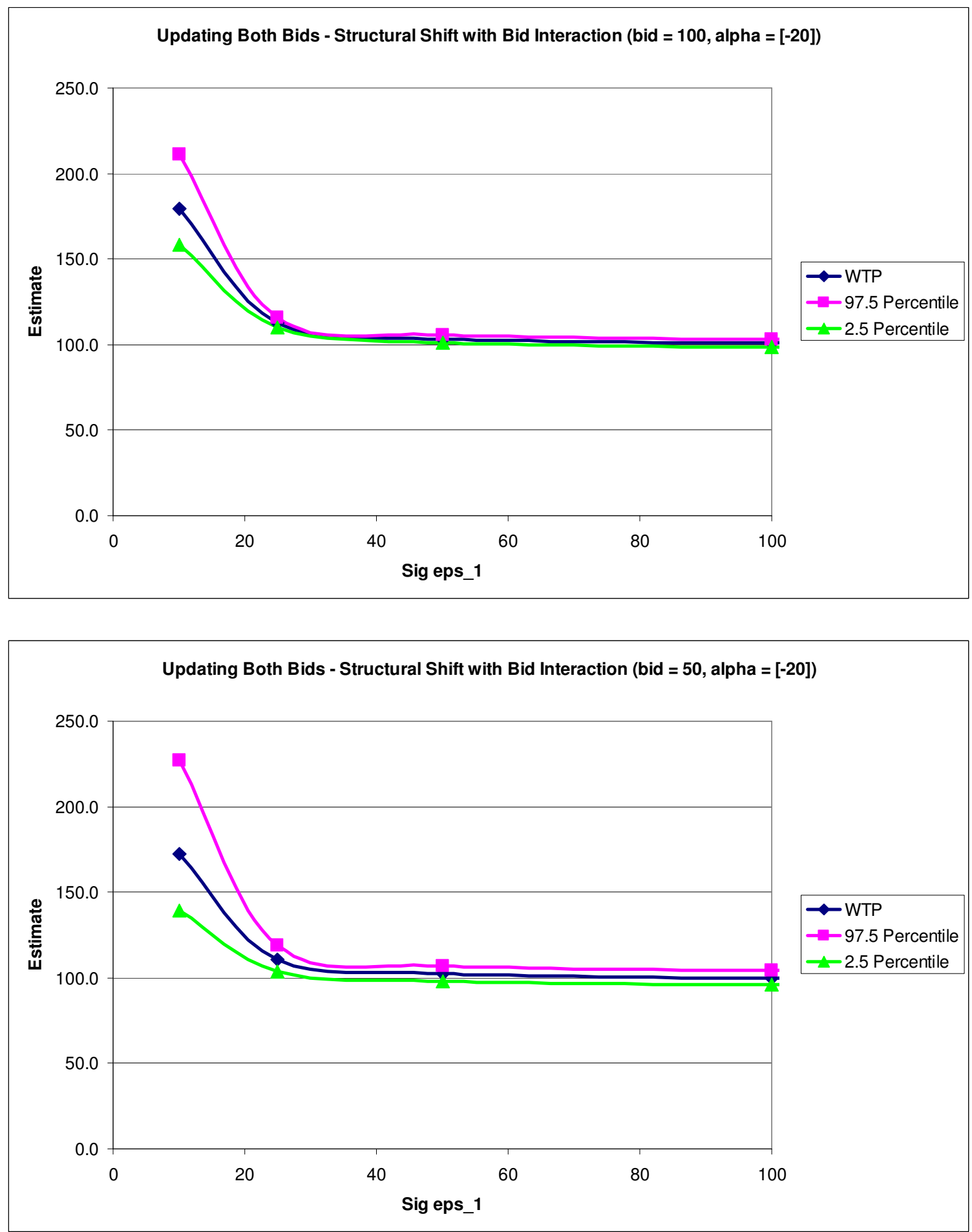


\section{DOES WATER QUALITY MATTER TO SOUTH FLORIDA HOMEBUYERS? EVIDENCE FROM A HEDONIC ANALYSIS}

\section{III.I. Introduction}

Hedonic property value models are used to determine whether a relationship exists between an environmental amenity and housing prices, and if so, to impute implicit prices for the environmental amenity that can be used for welfare analysis. Although hedonic property value studies that utilize air or noise quality as the environmental amenity in the empirical analysis are more prevalent, a number of fairly recent studies that employ water quality (WQ) as the environmental amenity have been applied including Boyle et al. (1999), Leggett and Bockstael (2000), Michael et al. (2000), Poor et al. (2001), and Gibbs et al. (2002). The purpose of this paper is to add to this WQ literature by capitalizing on a unique WQ dataset for a year-round, urban, and coastal housing market in Martin County, Florida. A major concern of this study is to assess the sensitivity of imputed valuations to the choice of WQ measure.

Often, hedonic property studies are limited to available environmental measures that may not be related to homeowners' perceptions (Michael et al. 2000). Additionally, there are questions as to whether homebuyers are incorporating a more scientific but less easily understood objective measure of water quality, or a less scientific but more easily understood subjective measure of WQ into their purchase decision (Epp and Al-Ani, 1979; Michael et al. 2000; Poor et al., 2001). The nature of our unique dataset allows us to effectively deal with these issues. Six WQ measures (temperature, $\mathrm{pH}$, water visibility, salinity, dissolved oxygen, and location grade) have been produced and documented weekly since 1998 for nine separate monitoring locations in our study area, 
and these measures are readily available to homeowners/buyers via the local newspaper and the internet. The sixth WQ measure, location grade, is a more easily understandable yet scientifically based objective measure of water quality with an A, B, C, D, or F letter grade and accompanying percentage $(90-100 \%, 80-90 \%, 70-80 \%, 60-70 \%$, and $<60 \%$ respectively) being assigned to each location depending upon the realized values of the five other objective WQ measures. In this way, results from the pure objective WQ measures are contrasted with the more easily understood yet objectively based WQ measure of location grade.

Boyle et al. (1999), Michael et al. (2000), Poor et al. (2001), and Gibbs et al. (2002) all demonstrate that the minimum water clarity value for the year of the home sale matters for Maine and New Hampshire lakefront homebuyers. However, these results are not easily transferable to an urban coastal housing market such as that of Martin County, FL. Here, not only are the water body and urban vs. rural settings different, but so is the timing of sales, which occur year-round and not just primarily in the summer months. Given a year-round housing market, a homebuyer focus on a minimum water clarity value for the year of the sale may not be the most appropriate as it plausibly could be for a mostly summer housing market in the Northeastern United States ${ }^{17}$. Therefore, in addition to employing minimum WQ values in the year of sale, we also incorporate other summary statistics that might matter in a year-round housing market such as the maximum, median, and statistical range.

\footnotetext{
${ }^{17}$ Leggett and Bockstael (2000) show that median fecal coliform for the year of the sale matters for a mixed urban and rural estuary/coastal setting in Maryland, but their data is also limited from April to September.
} 
In order to assess whether our various water quality measures affect waterfront housing prices in Martin County, FL we run pooled as well as time/location fixed effect regressions for our unbalanced panel dataset. Results indicate that our various forms of water quality do in fact matter to Martin County waterfront homebuyers. These results alone are especially relevant given the $\$ 1$ billion Indian River Lagoon South (IRLS) Everglades restoration effort being conducted to improve water quality in this area, and the lack of any formal economic benefit analysis conducted to-date (USACE/SFWMD, 2002). However, similar to Michael et al. (2000) no consistent relationship by either any of the six particular water quality variables, or by any of the four particular descriptive statistics used is found. This further clearly indicates that more efforts need to be aimed at understanding what particular WQ variable and its statistical form homebuyers use in making their purchase decision if hedonic property models utilizing WQ measures are to be applied successfully.

This paper is organized as follows: Section II provides an overview of the hedonic pricing theory and various water quality measures; Section III presents the compilation of the study data used; Section IV gives the empirical model and predictions while Section V gives the results; Section VI has the concluding comments.

\section{III.II. Background}

Hedonic Pricing Theory ${ }^{18}$

The underlying premise behind hedonic pricing theory is that the value of some market differentiated good is a function of each of its attributes. In this way, the market

\footnotetext{
${ }^{18}$ We present a brief overview here, for a more detailed overview please see either Freeman (2003) or Taylor (2003).
} 
differentiated good is able to serve as a "revealed" market for each of its attributes, with the price differences of the heterogeneous market good indicating implicit prices for the varying levels of its attributes. The hedonic price function (HPF) mathematically captures this relationship by expressing the price of a differentiated good as a function of its attributes whereby the partial derivative of the HPF with respect to any of its attributes gives the marginal implicit price (hedonic price) of the attribute.

Hedonic pricing theory can be applied to the housing market through the hedonic property value model. Under a number of assumptions, including a single market for housing where the HPF can be thought of as the locus of equilibria between housing bids (demand) and housing offers (supply), the rental price of the jth residential location can be taken to be a function of structural, neighborhood, and environmental amenities such that:

$$
R_{j}=R\left(S_{j}, N_{j}, Q_{j}\right)
$$

where $R(\bullet)$ is the hedonic price function, $S_{j}$ is a vector of structural characteristics, $N_{j}$ is a vector of neighborhood characteristics, and $Q_{j}$ is a vector of location-specific environmental amenities. Here, letting $q$ be an element of $Q_{j}, \partial R / \partial q$ is the marginal implicit price of the environmental amenity selected. It can be shown from a utility maximization framework that the derived hedonic prices are equal to an individual's maximum willingness-to-pay, and hence it is possible to use them to conduct welfare $\operatorname{analysis}^{19}$.

\footnotetext{
${ }^{19}$ A separate second-stage estimation is usually required in order to derive the complete MWTP function necessary for an appropriate welfare analysis.
} 


\section{Water Quality}

Direct effects on a water body due to poor WQ levels are decreased light availability, algal dominance changes, and increased organic matter decomposition. These direct effects can in turn lead to other significant indirect effects such as loss of submerged aquatic vegetation, harmful algae blooms, and low dissolved oxygen levels (Bricker et al., 1999). The direct and indirect effects due to poor WQ can then result in significant adverse impacts to a water body such as loss of habitat, increase of algal toxins, fish kills, and offensive odors which would subsequently impair use and aesthetic values (Bricker et al., 1999) ${ }^{20}$. For example, fish kills would curtail use of the water body for any commercial or recreational fishing, while offensive odors would most likely lower any aesthetic values or tourist related activities.

There are various WQ measures used in order to monitor the health of a water body including temperature, $\mathrm{pH}$, water visibility, salinity, and dissolved oxygen. The Florida Oceanographic Society (FOS, 2004) provides an overview of each of these measures:

Temperature - "dramatically affects the rates of chemical and biological reactions" including "the solubility of chemical compounds in water, distribution and abundance of organisms, rate of growth of biological organisms, water density, mixing of different water densities, and current movements". Inversely related to dissolved oxygen, and interrelated to salinity. Undergoes wide seasonal variations.

$p H$ - measure of the acidity or excess alkali of a water body where on a scale of 1 to 14,7 is neutral, $<7$ is acidic, and $>7$ has excess alkali. "Affects the solubility of minerals in water. The buffering capacity of water, its ability to resist changes in $\mathrm{pH}$, is critical to aquatic life", where aquatic organism survival greatly

\footnotetext{
${ }^{20}$ Bricker et al. (1999) are specifically referring to eutrophication (i.e., the result of excessive organic matter loading) of a waterbody causing these direct, indirect, and hence adverse impacts. For ease of exposition here, I interchange poor WQ levels for eutrophication.
} 
decreasing with $\mathrm{pH}<5$ or $\mathrm{pH}>9$. Most of the extreme situations will be found in low salinity situations.

Water Visibility (Water Clarity) - "material that becomes mixed or suspended in water will cause the water to become more turbid and reduce the clarity of the water. As the water clarity decreases, light will not be able to penetrate as far below the water's surface. If light levels become too low, photosynthesis of plants below the water may stop, and the plants will die. These plants produce oxygen and habitat for aquatic life." Secchi disks are typically used to measure visibility levels.

Salinity - "the concentration of dissolved salt in water normally expressed in parts per thousand. While in any given location salinity levels will vary, extreme salinity changes can affect the well-being and distribution of biological populations". Undergoes wide seasonal variations.

Dissolved Oxygen $(D O)$ - "one of the most important indicators of water quality, as it is essential for the survival of fish and other aquatic life. When levels are too low, aquatic life cannot survive. The colder the water is, the more oxygen it can hold and vice versa. Also, as water becomes more fresh (lower salinity), more oxygen can dissolve into the water. Fish kills occur with DO $<3 \mathrm{mg} / \mathrm{L}$." Exhibits high seasonal as well as daily variations stemming from its inverse relationship to temperature.

Overall, these five WQ measures can be used to monitor the health of a water body, but they are plainly interrelated and also susceptible to wide seasonal variations.

\section{III.III. Study Area and Associated Data}

Study Area

Martin County is located on the Southeastern Atlantic coast of Florida. Figure 1 indicates the Northeastern portion of the county and its accompanying waterfront housing market located on the St. Lucie River, St. Lucie Estuary, and Indian River Lagoon, which is the housing market analyzed for this study. Analogous to Leggett and Bockstael (2000), this area is well-suited for a hedonic analysis of water quality due to the substantial and sufficiently varied number of waterfront properties, the lively housing 
market in the area, as well as the variation in water quality across the St. Lucie River, St. Lucie Estuary, and the Indian River Lagoon.

The specific home sale data used for the analysis are sales of Martin County waterfront properties from January 2000 to August 2004 as supplied by the Martin County property appraiser's office. The sales price is adjusted to $2^{\text {nd }}$ quarter 2004 values $^{21}$ using the Office of Federal Housing Enterprise Oversight (OFHEO, 2004) housing index for the Fort Pierce-Port St. Lucie Metropolitan Statistical Area. ${ }^{22}$ After deleting a total of 288 sales from the original data set due to missing sale dates/sales prices, non-single family homes, missing structural data, structural data that is dated after the date of the sale, missing water quality data, and otherwise strange-looking data ${ }^{23}$, a total of 743 observations remained.

509 of the homes were sold exactly once over this timeframe, with 108 homes sold two or more times, with a maximum of four sales. Each of the sales is inputted by parcel into a geographical information systems (GIS) database, with each parcel converted into a point as depicted in Figure 2. Table 1 presents the number of waterfront sales per month from January 2000 to August 2004. Clearly the waterfront housing market in Martin County is a year-round market with no one month accounting for more

\footnotetext{
${ }^{21}$ Despite having July and August 2004 sales data, a $2^{\text {nd }}$-quarter index is chosen (the last complete quarter of home sales) such that April through August 2004 home sale prices were not adjusted.

${ }^{22}$ It should be noted that this index did experience a significant overall increase of $56 \%$ between $3^{\text {rd }}$ quarter 2001 and $2^{\text {nd }}$ quarter 2004 as compared to a $15 \%$ increase from $4^{\text {th }}$ quarter 1998 to $3^{\text {rd }}$ quarter 2001 , a prior but similar length of time. These results do raise some concerns in regard to the equilibrium assumptions of the hedonic model as described in section II.

${ }^{23}$ This included separate sales booked on the same day or within 1-2 days with significant decreases in sale price; extremely low sales prices; sales price - appraised value $<0$.
} 
than $13.5 \%$ of the total waterfront sales during any one year, and no one month accounting for more than $11.5 \%$ of the total waterfront sales during the study timeframe.

\section{Water Quality}

Water quality data for the Martin County waterfront area is tracked on a weekly basis by the Florida Oceanographic Society (FOS, 2004) for nine separate locations as depicted in Figure 3. Beside the public availability of data on the FOS website, this data is also published weekly in local newspapers and therefore it is reasonable to consider this a known, important, and readily available source of water quality data. The specific water quality locations tracked and used for this study include 2. North Fork, 3. South Fork, 5. Wide Middle River, 6. Narrow Middle River, 7. Manatee Pocket, 8. Inlet Area, and 9. IRL. ${ }^{24}$

Figure 4 presents an example of the weekly WQ data available by location. A total of five distinct objective water quality variables are collected and published weekly - temperature, $\mathrm{pH}$, water visibility, salinity, and dissolved oxygen (DO); as well as one objectively based compiled measure of water quality - location grade. Furthermore, all of the distinct objective water quality measures, excluding temperature, are briefly explained in the published data and given corresponding labels of poor, fair, and good over specified ranges of values. All six of the published numeric WQ measures are used as inputs for the hedonic analysis with data collected from January 2000 to December 2004. As can be seen in Figure 4, not all locations report WQ data every week.

\footnotetext{
${ }^{24}$ Location 1. Winding North Fork is a part of St. Lucie County for which home sales were not collected, and no home sales from the original data were provided for location 4. Winding South Fork
} 
Significant gaps occurred with 6. Narrow Middle River, 7. Manatee Pocket, 8. Inlet Area, and 9. IRL in 2004, as well as 8. Inlet Area for 2000, 2001, and 2003. Consequently, corresponding location waterfront home sales during these time periods were excluded from the analysis.

Section II indicated that the above five distinct WQ measures are interrelated and are susceptible to wide seasonal variations. Correlation analysis results based upon all of the weekly data used in the analysis are shown in Table 2 where correlations $>|0.5|$ are highlighted. As expected, a strong negative correlation exists between temperature and DO, while a strong positive correlation exists between $\mathrm{pH}$ and salinity. Strong correlations also exist between salinity and water visibility, as well as between location grade, water visibility, and salinity. Figure 5 illustrates the seasonal and locational variations of the data for the study period, using the weekly changes in DO levels by location as an example.

Each home sale is manually assigned to a water quality location by viewing the actual location of the home through the use of both the constructed GIS database and Martin County property appraiser website (MCPA, 2004) overlaid upon the given water quality location map of Figure 3. Figure 6 presents the home sales as per their assigned WQ locations. The associated WQ data for that location is then assigned to each home by the date of the sale matched to the weekly WQ publication date. In this way, all homes assigned to the same location receive the same annual WQ data for an equal sale year. For example, if one home located on the western end of location 5 Wide Middle River sold in 2000, and another home located on the eastern end of location 5 (near to location 6) also sold in 2000, equal annual WQ values would be assigned to each home 
despite the distances between the two homes. There are 16 homes located directly in the middle of the North and South Forks that are assigned to a created location of 2.5 with associated WQ data taken to be the average of the two North and South Forks locations.

Sufficient variation exists in the WQ data across locations. For example, Figure 7a illustrates the variation in the median annual values of water visibility by location over time, while Figure $7 \mathrm{~b}$ illustrates variation in the minimum, average, and maximum water visibility values by location for the entire study timeframe. Similar variation exists for the other five WQ measures. Given the year-round housing market for our study area, it is also useful to understand where the timing of minimum and maximum values for our WQ measures occur throughout the year. Table 3 illustrates that for North Fork the minimum annual values for DO occur in the summer months while the maximum annual values occur in the winter months as would be expected given the inverse relationship between water temperature and DO. Similar timing of maximum and minimum WQ values exists for the other WQ locations.

Importantly though, we do not know exactly when homeowners saw the location for the first time, or what particular statistical descriptor they tend to focus on in their purchase decision. Because of this, including only the minimum values in the empirical analysis may not truly reflect a homebuyer's decision making efforts in a year-round housing market. Therefore, we include the statistical descriptors of the minimum, median, maximum, and statistical range for our WQ measures in the empirical analysis. 


\section{Structural, Neighborhood, and Macroeconomic}

Structural and neighborhood characteristics chosen are based on a review of previous studies as well as availability of data. Structural variables included in the estimation are square footage of both land and house, age of the house, and the number of bathrooms. House size and age of the home are also squared to allow for non-linearity in these variables. A series of dummy variables are also included to indicate whether the house has a pool, boat lift, dock, etc ${ }^{25}$. Other dummies indicated whether the house had more than one structure ${ }^{26}$, whether the house had been sold more than once during the time period, and notably the type of exterior wall ( $1=$ concrete block) due to the frequency of hurricanes in Florida.

Three neighborhood variables are selected in order to capture ethnicity (percent white), age (percent older than 65), and socio-economic demographics (percent of household owners). This neighborhood information is obtained from the 2000 census data (FGDL, 2004), and using the constructed GIS database, each home is spatially joined to its corresponding census tract level. Lastly, we also include the mortgage interest rate ${ }^{27}$ which is commonly thought to be one of the main drivers of housing sales and prices. Despite the significance of interest rates in purchasing a home, this is typically not included in the environmental hedonic models reviewed in the literature. Table 4 provides descriptive statistics for each of the variables included in the empirical

\footnotetext{
${ }^{25}$ These data were listed under improvements on the property appraiser website and no date for the improvement was given. Therefore, it was assumed that if an improvement was listed it was there as the time of sale.

${ }^{26}$ If the home did have more than one structure, the square footage and number of baths were summed and the other dummy variables were taken from the largest structure listed.

${ }^{27}$ Monthly national interest rate for a 30 year fixed rate mortgage as provided by HSH Associates (HSH, 2004)
} 
analysis, while Table 5 provides definitions along with their expected signs in the hedonic regressions.

\section{III.IV. Model Estimation}

We select a semi-log functional form for our model estimation with the natural $\log$ of sales price taken to be the dependent variable. A common assumption of hedonic property models is that data from one specific urban area, such as our study data, represents a single geographic market (Taylor, 2003). In this way, separate ordinary least squares regressions are repeated for each measure of WQ for our pooled dataset. However, location and time aspects of a hedonic dataset are important since if in fact location and/or time submarkets exist within the data, estimating a single HPF for that market will lead to incorrect parameter estimates and imputed prices (Freeman, 2003). So, while our study data comes from a relatively distinct area of Martin County, we can easily incorporate location and time dummies to account for any possible location and/or time submarkets existing within our unbalanced panel dataset.

We create $N=3$ location dummies based upon the three distinct waterbodies of the study area and their associated WQ monitoring locations such that individual home sales occurring in locations 2. North Fork, 3. South Fork, and the created location of 2.5 correspond to a river location submarket; locations 5. Wide Middle River, 6. Narrow Middle River, and 7. Manatee Pocket correspond to an estuary location submarket; and locations 8. Inlet Area and 9. IRL correspond to a lagoon location submarket. ${ }^{28} T=5$ time

\footnotetext{
${ }^{28}$ Given that our data is already from a relatively small geographical area, grouping by the main waterbody types seemed reasonable for a submarket breakdown vs. having a separate submarket for each individual WQ location. Futhermore, WQ and housing data across the defined submarkets is similar.
} 
dummies are simply for each possible sale year from 2000 to 2004 . By adding $(N-1)$ location and (T-1) time intercept dummies the following fixed-effect model ${ }^{29}$ is created for our unbalanced panel dataset to again be estimated by ordinary least squares:

$$
\begin{aligned}
\ln (\text { Price 04 })_{i t}= & \alpha+\delta_{1} D_{\text {River }}+\delta_{2} D_{\text {Estuary }}+\lambda_{1} D_{2000}+\lambda_{2} D_{2001}+\lambda_{3} D_{2002}+\lambda_{4} D_{2003} \\
& +\beta_{1} \text { LSqFt }_{i t}+\beta_{2} \text { HSqFt }_{i t}+\beta_{3} \text { HSqFt }_{i t}^{2}+\beta_{4} \text { Age }_{i t}+\beta_{5} \text { Age }^{2}{ }_{i t} \\
& +\beta_{6} \text { Bath }_{i t}+\beta_{7} \operatorname{Re} \text { Sale }_{i t}+\beta_{8} \text { Struct }_{i t}+\beta_{9} \text { ExtrW }_{i t}+\beta_{10} \text { Fire }_{i t} \\
& +\beta_{11} \text { Enclsr }_{i t}+\beta_{12} \text { Pool }_{i t}+\beta_{13} \text { BLift }_{i t}+\beta_{14} \text { Dock }_{i t}+\beta_{15} \text { Special }_{i t} \\
& +\beta_{16} \text { WQ }_{i t}+\beta_{17} \text { White }_{i t}+\beta_{18} 65_{i t}+\beta_{19} \text { Own }_{i t}+\beta_{20} \text { Int }_{i t}+\varepsilon_{i t}
\end{aligned}
$$

where $D_{\text {River }}=1$ if the observation belongs to the river submarket, 0 otherwise; $D_{\text {Estuary }}=1$ if the observation belongs to the estuary submarket, 0 otherwise; $D_{2000}=1$ for an observation in year 2000, 0 otherwise; $D_{2001}=1$ for an observation in year 2001, 0 otherwise; $D_{2002}=1$ for an observation in year 2002, 0 otherwise; $D_{2003}=1$ for an observation in year 2003, 0 otherwise; and the dummy variables for the lagoon location and the year 2004 are excluded in order to avoid perfect multicollinearity issues.

\section{III.V. Results}

Pooled Model

Results of the pooled regression are given in Tables $6,7,8$, and 9 for the minimum, median, statistical range, and maximum WQ measures respectively. BreuschPagan test statistics for all regressions indicate the presence of heteroscedasticity in the data. This result was expected due to an initial scatterplot analysis that was conducted of explanatory variables vs. sales price, which pointed to the likely presence of

\footnotetext{
${ }^{29}$ A formal Hausman test for fixed effect vs. random effect model was not conducted. Given our small number of cross-sectional and time units, the straightforward fixed-effect approach was preferred.
} 
heteroscedasticity in the data, as well as the frequent finding of heteroscedasticity from the literature (Leggett and Bockstael, 2000), (Gibbs et al., 2002), (Stevenson, 2004). Hence, White's robust $t$-statistics are used.

All models explain approximately 56\% of the variation in the Martin County waterfront housing sales prices. Across all specifications of the different WQ measures, housing square footage, housing square footage squared, interest rates, and the dummy variables for whether the property sold more than once over the data time period, a pool/patio enclosure, an in-ground pool, a boat lift, and a waterfront dock are consistently significant at the $1 \%$ level with both of the housing square footage variables, interest rates and the dummies for pool, boat lift, and dock having the expected signs. There were no prior expectations on the sign of whether a home sold more than once over our study time-period, but the negative sign on pool/patio enclosure was not expected. Notably, the significance of pool, boat lift, and dock variables highlights the importance of water-related structural variables to housing prices, and hence plausibly the importance of good WQ in order to better enjoy any water-related activities associated with these water-related structural variables. One could argue that housing square footage is capturing the significance of other related structural variables one would expect to be significant such as land square footage and the number of bathrooms. Given the prominence of hurricane threats in this area, the non-significance of our exterior wall dummy variable is surprising. The fact that none of our neighborhood variables is consistently significant is also unexpected, although the percentage of the population older than 65 is significant at the $10 \%$ level in some of the regressions. 
As the semi-log functional form is used for estimation, the estimated coefficient is interpreted as the percentage change in the mean sales price for an additional unit of the variable. The existence of one of the water-related structural variables increases a home's value in our study area by approximately $10-12 \%$ per structure. Likewise, a $1 \%$ increase in interest rates results in a $14-17 \%$ drop in the mean sale price. The mean sale price is used to derive hedonic prices from our regression coefficients. For example, the derived hedonic price for one square foot of home across all models is approximately $(0.54 / 1000) * \$ 868,000=\$ 468$ per square foot. This derived amount is relatively close to the average cost per square foot of home from Table 5 of $\$ 333$. Excluding the coefficient estimates for a pool/patio enclosure, estimated coefficients and derived hedonic prices for our other significant structural and neighborhood variables are reasonable.

While our coefficient estimate results for the structural and neighborhood variables are consistent across the various WQ variables specifications, the WQ coefficient estimate results are not. Table 10 illustrates the inconsistency of the significance of the WQ measures depending upon which statistical descriptor is being utilized. For example, while we also find that water visibility is significant when the minimum value as per the year of the sale is the statistical descriptor used in the regression as did Boyle et al. (1999), Michael et al. (2000), Poor et al. (2001), and Gibbs et al. (2002), it is not significant when the median or statistical range descriptors are used, and also not as significant (1\% vs. $5 \%$ levels) when the maximum value as per the year of the sale is used. Similar results emerge for the other WQ measures in that none is significant across all statistical descriptors, and also that the strength of significance varies by descriptor. No WQ measures are significant when the statistical range 
descriptor is utilized, and $\mathrm{pH}$ is not significant in any model. Only water visibility is significant when minimum values are used, while three of the six measures and five of the six measures are significant when median and maximum values are used respectively. Importantly, these results show that the significance of the WQ measure used is dependent upon the statistical descriptor chosen in the empirical analysis.

Table 11 presents the derived hedonic prices for our significant WQ measures. All signs are correct except for location grade using the median value of the year of the sale and DO for the maximum value of the year of the sale. The derived hedonic prices vary from $0.3 \%$ to $3.5 \%$ of the total home value and are comparable to those derived from previous studies. These hedonic prices can potentially be used as an input to a welfare analysis of the IRLS restoration effort.

Finally, location grade does not provide any additional insight over the other objective WQ measures. While it is significant in the regressions using the median and maximum statistical descriptors for our WQ measures, so too are the other measures it is highly correlated with, salinity and water visibility. Also, its derived hedonic prices are in-line with the other derived hedonic prices, even having an unexpected negative coefficient for the median annual value.

\section{Fixed Effect Model}

Adjusted $\mathrm{R}^{2}$ values indicate our fixed effect models explaining approximately $58 \%$ of the variation in the Martin County waterfront housing sales prices. While this is not a radical improvement over the pooled model results, restricted $F$-tests indicate that the restricted pooled regressions are invalid at the $1 \%$ level. Breusch-Pagan test statistics 
for all regressions again indicate the presence of heteroscedasticity in the data, and consequently White's robust $t$-statistics are once more used.

As the structural and neighborhood variable coefficient results from the fixed effect models are very similar to the pooled model results, complete model results are not presented here. Once again, across all specifications of the different WQ measures, housing square footage, housing square footage squared, interest rates, and the dummy variables for whether the property sold more than once over the data time period, a pool/patio enclosure, an in-ground pool, a boat lift, and a waterfront dock are consistently significant at the $1 \%$ level with both of the housing square footage variables, interest rates and the dummies for pool, boat lift, and dock having the expected signs. Coefficient estimates reconfirm that the existence of one of the water-related structural variables increases a home's value in our study area by approximately $10-12 \%$ per structure. One notable exception between the pooled and fixed effect models is the significance of some of the neighborhood explanatory variables. The fixed effect models have the percentage of the population older than 65 and the percentage of owner occupied homes as being consistently significant at the $10 \%$ level or higher for nearly all of the estimated models. This neighborhood variable result further substantiates the inclusion of the location submarket dummy variables.

Results of the fixed effect regressions for the WQ variables and the location and time dummy variables are presented in Tables 12, 13, 14, and 15 for the minimum, median, statistical range, and maximum WQ measures respectively. $T$-statistics indicate that the location submarkets are significant. Coefficient estimates on the dummy variables for a river location are consistently significant at the $1 \%$ level and negative. 
Coefficient estimates on the dummy variable for an estuary location are not as consistently significant as those of the river location dummy, but generally are significant at the 5\% level and positive. Time submarket significance exists primarily for years 2002 and 2003, but is clearly not consistent across estimations.

Table 16 illustrates a similar WQ measure significance inconsistency as we saw with our pooled model results of Table 10. While DO and Grade are significant across nearly all statistical descriptors used, the same results do not hold for the remaining WQ variables. For example, water visibility is highly significant when median values as per the year of the sale are used, but is an insignificant variable for all other statistical descriptors used. Our fixed effect results again importantly show that the significance of the WQ measure used is dependent upon the statistical descriptor chosen in the empirical analysis.

While the derived hedonic prices across the pooled and fixed effect models are similar, there are some important differences between our pooled and fixed effect WQ results. One troubling difference is that coefficient estimates in the fixed effect models are mainly negative, while coefficient expectations for both models and the actual pooled model results are mostly positive. Another notable result is that regressions using the distribution of the WQ variable in the year of the sale indicate the significance of the WQ variables in the fixed effect model, while these were entirely insignificant in the pooled model results. Also, WQ variables are significant for different descriptors depending upon the model. For example, in the pooled model salinity is significant for the median and maximum values, but in the fixed effect model it is now significant for the minimum and distribution values. Lastly, location grade appears to play a more important role in 
the fixed effect regressions as it is significant in three of the four statistical descriptor estimations. However, its sign in each regression is inconsistent with expectations.

\section{III.VI. Conclusions}

We capitalize on a unique WQ dataset for a year-round, urban, and coastal housing market in Martin County, Florida and through the use of a hedonic property value model find evidence that various WQ measures affect waterfront housing prices in this setting. However, our results indicate that this relationship is not consistent across any of the six particular WQ variables used, and is furthermore importantly dependent upon the specific descriptive statistic employed to represent the WQ measure in the empirical analysis. Additionally, while our dataset contains a more easily understood yet scientifically based objective measure of water quality (i.e., location grade), no distinct advantage is gained in using this measure over a traditional pure objective measure of water quality that may not be as easily understood by homebuyers such as dissolved oxygen. Our results continue to advance the current notion in the WQ hedonic literature that more efforts need to be aimed at understanding what particular WQ variable and its statistical form homebuyers use in making their purchase decision if hedonic property models utilizing WQ measures are to be applied successfully. 
Table III.1. Waterfront Sales by Month from Jan 2000 to Aug 2004

\begin{tabular}{|c|c|c|c|c|c|c|c|}
\hline $\begin{array}{c}\text { Month } \\
\text { of Sale }\end{array}$ & $\mathbf{2 0 0 0}$ & $\mathbf{2 0 0 1}$ & $\mathbf{2 0 0 2}$ & $\mathbf{2 0 0 3}$ & $\mathbf{2 0 0 4}$ & Total & $\begin{array}{c}\text { \% of } \\
\text { Total }\end{array}$ \\
\hline 1 & 10 & 8 & 17 & 10 & 2 & 47 & $6.3 \%$ \\
\hline 2 & 15 & 10 & 13 & 14 & 12 & 64 & $8.6 \%$ \\
\hline 3 & 21 & 14 & 15 & 11 & 8 & 69 & $9.3 \%$ \\
\hline 4 & 18 & 21 & 22 & 11 & 10 & 82 & $11.0 \%$ \\
\hline 5 & 19 & 15 & 26 & 9 & 16 & 85 & $11.4 \%$ \\
\hline 6 & 20 & 11 & 14 & 8 & 7 & 60 & $8.1 \%$ \\
\hline 7 & 10 & 18 & 17 & 9 & 4 & 58 & $7.8 \%$ \\
\hline 8 & 12 & 17 & 22 & 9 & 7 & 67 & $9.0 \%$ \\
\hline 9 & 11 & 12 & 13 & 11 & & 47 & $6.3 \%$ \\
\hline 10 & 11 & 14 & 13 & 13 & & 51 & $6.9 \%$ \\
\hline 11 & 19 & 7 & 16 & 9 & & 51 & $6.9 \%$ \\
\hline 12 & 19 & 9 & 14 & 20 & & 62 & $8.3 \%$ \\
\hline Total & $\mathbf{1 8 5}$ & $\mathbf{1 5 6}$ & $\mathbf{2 0 2}$ & $\mathbf{1 3 4}$ & $\mathbf{6 6}$ & $\mathbf{7 4 3}$ & $\mathbf{1 0 0 \%}$ \\
\hline
\end{tabular}

Table III.2. Correlation of WQ Measures

\begin{tabular}{|lcccccc|}
\hline & $\begin{array}{c}\text { Water } \\
\text { Temp. }\end{array}$ & $p H$ & $\begin{array}{c}\text { Water } \\
\text { Visibility }\end{array}$ & Salinity & $\begin{array}{c}\text { Dissolved } \\
\text { Oxygen }\end{array}$ & $\begin{array}{c}\text { Location } \\
\text { Grade }\end{array}$ \\
\hline Water Temp. & 1 & & & & & \\
pH & -0.08 & 1 & & & & \\
Water Visibility & -0.05 & 0.32 & 1 & & & \\
Salinity & -0.08 & 0.54 & 0.63 & 1 & & \\
Dissolved Oxygen & -0.50 & 0.34 & 0.01 & 0.00 & 1 & \\
Location Grade & -0.21 & 0.44 & 0.63 & 0.59 & 0.21 & 1 \\
\hline
\end{tabular}


Table III.3. DO Levels Statistical Descriptors by Month for Location 2. North Fork

\begin{tabular}{|c|c|c|c|c|c|}
\hline $\begin{array}{c}\text { Month } \\
\text { of Sale }\end{array}$ & $\mathbf{2 0 0 0}$ & $\mathbf{2 0 0 1}$ & $\mathbf{2 0 0 2}$ & $\mathbf{2 0 0 3}$ & $\mathbf{2 0 0 4}$ \\
\hline 1 & & Max & Max & Max & \\
\hline 2 & & & & & \\
\hline 3 & & & & & \\
\hline 4 & & & & & \\
\hline 5 & & & & & \\
\hline 6 & & & & & \\
\hline 7 & & Min & Min & & \\
\hline 8 & Min & & & & \\
\hline 9 & & & & Min & Min \\
\hline 10 & & & & & \\
\hline 11 & & & & & \\
\hline 12 & Max & & & & Max \\
\hline
\end{tabular}


Table III.4. Data Descriptive Statistics

\begin{tabular}{|c|c|c|c|c|}
\hline Variable & Mean & Stnd Deviation & Minimum & Maximum \\
\hline Sales Price $(\$ 000$ 's) & 868.0 & 791.3 & 68.1 & 7224.5 \\
\hline Land SqFt (000's) & 24.9 & 39.0 & 1.0 & 883.7 \\
\hline Home SqFt (000's) & 2.6 & 1.4 & 0.4 & 13.4 \\
\hline Age & 26.4 & 14.7 & 0.0 & 100.0 \\
\hline Bath & 2.8 & 1.2 & 1.0 & 10.0 \\
\hline Repeat Sale & 0.34 & & & \\
\hline$>1$ Structure & 0.05 & & & \\
\hline Exterior Wall & 0.49 & & & \\
\hline Fireplace & 0.37 & & & \\
\hline Enclosure & 0.22 & & & \\
\hline Pool & 0.52 & & & \\
\hline Boat Lift & 0.31 & & & \\
\hline Dock & 0.73 & & & \\
\hline Special & 0.29 & & & \\
\hline Water Temperature & 75.9 & 2.7 & 71.6 & 80.6 \\
\hline $\mathrm{pH}$ & 8.0 & 0.1 & 7.8 & 8.2 \\
\hline Water Visibility (\%) & 55.5 & 20.9 & 31.2 & 100.0 \\
\hline Salinity (ppt) & 17.4 & 8.6 & 1.0 & 34.0 \\
\hline Dissolved Oxygen & 6.3 & 0.5 & 5.3 & 7.7 \\
\hline Location Grade (\%) & 83.8 & 9.9 & 63.0 & 100.0 \\
\hline$\%$ White & 95.4 & 6.6 & 41.7 & 99.1 \\
\hline$\% 65$ & 33.5 & 8.9 & 14.8 & 51.3 \\
\hline$\%$ Owner & 80.9 & 8.9 & 36.6 & 92.6 \\
\hline IntRate (\%) & 7.0 & 0.9 & 5.4 & 8.7 \\
\hline
\end{tabular}

Note: WQ Measure data are for the annual median values 
Table III.5. Names and Descriptions of Variables

\begin{tabular}{|c|c|c|c|c|c|}
\hline Variable & Description & \multicolumn{4}{|c|}{ Expected Sign } \\
\hline Price04 & Sales price of home adjusted to Q2 2004 value & & & & \\
\hline $\mathrm{LSqFt}$ & Total Square footage of land parcel & \multicolumn{4}{|c|}{+} \\
\hline $\mathrm{HSqFt}$ & Total housing square footage & \multicolumn{4}{|c|}{+} \\
\hline $\mathrm{HSqFt}^{2}$ & Total housing square footage squared & \multicolumn{4}{|c|}{-} \\
\hline Age & Age of home $=($ Year of sale - Year built $)$ & \multicolumn{4}{|c|}{ - } \\
\hline $\mathrm{Age}^{2}$ & Age of home squared & \multicolumn{4}{|c|}{-} \\
\hline Bath & $\begin{array}{l}\text { Total \# of bathrooms = \# of Full baths + \# of half } \\
\text { baths }\end{array}$ & \multicolumn{4}{|c|}{+} \\
\hline ReSale & $\begin{array}{l}\text { Dummy variable for whether the property sold } \\
\text { more than once over the data time period } \\
\text { (1=2 or more sales of same home, } 0=1 \text { sale only) }\end{array}$ & \multicolumn{4}{|c|}{$?$} \\
\hline Struct & $\begin{array}{l}\text { Dummy variable for }>1 \text { residence structure on the } \\
\text { property } \\
(1=>1 \text { residence structure, } 0=1 \text { residence } \\
\text { structure }\end{array}$ & \multicolumn{4}{|c|}{$?$} \\
\hline ExtrW & $\begin{array}{l}\text { Dummy variable for exterior walls } \\
(1=\text { concrete block, } 0=\text { otherwise })\end{array}$ & \multicolumn{4}{|c|}{+} \\
\hline Fire & $\begin{array}{l}\text { Dummy variable for fireplace } \\
(1=\text { Yes, } 0=\text { No })\end{array}$ & \multicolumn{4}{|c|}{+} \\
\hline Enclsr & $\begin{array}{l}\text { Dummy variable for pool/patio enclosure } \\
(1=\text { Yes, } 0=\mathrm{No})\end{array}$ & \multicolumn{4}{|c|}{+} \\
\hline Pool & $\begin{array}{l}\text { Dummy variable for in-ground pool } \\
(1=\text { Yes, } 0=\mathrm{No})\end{array}$ & \multicolumn{4}{|c|}{+} \\
\hline BLift & $\begin{array}{l}\text { Dummy variable for boat lift } \\
(1=\text { Yes, } 0=\mathrm{No})\end{array}$ & \multicolumn{4}{|c|}{+} \\
\hline Dock & $\begin{array}{l}\text { Dummy variable for waterfront dock } \\
(1=\text { Yes, } 0=\text { No) }\end{array}$ & \multicolumn{4}{|c|}{+} \\
\hline Special & $\begin{array}{l}\text { Dummy variable for home special feature } \\
\text { including carport, jacuzzi, BBQ, boathouse, } \\
\text { gazebo, tennis court, and fountain } \\
(1=\text { Yes, } 0=\text { No) }\end{array}$ & \multicolumn{4}{|c|}{+} \\
\hline \multirow{2}{*}{$\begin{array}{ll}\text { WQ: } \\
\text { - } \\
\text { - } \text { Wtemp } \\
\text { - } & \text { Wvis } \\
\text { - } & \text { Salinit } \\
\text { - } & \text { DO } \\
\text { - } & \text { Grade } \\
\end{array}$} & \multirow[b]{2}{*}{$\begin{array}{l}\text { Median, minimum, maximum and [maximum - } \\
\text { minimum] values by designated water quality area } \\
\text { for year of sale }\end{array}$} & Min & Med & $\begin{array}{l}{[\mathrm{Max}-} \\
\text { Min] }\end{array}$ & Max \\
\hline & & $\begin{array}{l}+ \\
+ \\
+ \\
+ \\
+ \\
+\end{array}$ & $\begin{array}{l}? \\
? \\
+ \\
+ \\
+ \\
+\end{array}$ & $\begin{array}{l}- \\
- \\
- \\
- \\
- \\
-\end{array}$ & $\begin{array}{l}- \\
- \\
+ \\
+ \\
+ \\
+\end{array}$ \\
\hline White & $\begin{array}{l}\% \text { of population that is white at the } 2000 \text { census } \\
\text { tract level }\end{array}$ & \multicolumn{4}{|c|}{+} \\
\hline 65 & $\begin{array}{l}\% \text { of population that is age } 65 \text { or over at the } 2000 \\
\text { census tract level }\end{array}$ & \multicolumn{4}{|c|}{ ? } \\
\hline Own & $\begin{array}{l}\% \text { of households that are owner occupied at the } \\
2000 \text { census tract level }\end{array}$ & \multicolumn{4}{|c|}{+} \\
\hline Int & $\begin{array}{l}\text { National } 30 \text { year fixed interest rate in month and } \\
\text { year of sale }\end{array}$ & \multicolumn{4}{|c|}{ 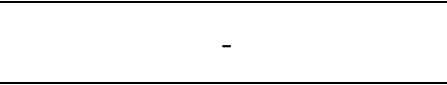 } \\
\hline
\end{tabular}


Table III.6. Pooled OLS Results Minimum WQ

\begin{tabular}{|c|c|c|c|c|c|c|}
\hline Variable & WTemp & pH & $\underline{W V i s}$ & $\underline{\text { Salinit }}$ & $\underline{\text { DO }}$ & Grade \\
\hline intercept & $\begin{array}{c}12.955 * * * \\
(31.612)\end{array}$ & $\begin{array}{c}12.313 * * * \\
(19.693)\end{array}$ & $\begin{array}{l}12.99 * * * \\
(37.873)\end{array}$ & $\begin{array}{c}13.055^{* * * *} \\
(38.432)\end{array}$ & $\begin{array}{c}13.066^{* * *} \\
(38.753)\end{array}$ & $\begin{array}{c}13.02 * * * \\
(38.14)\end{array}$ \\
\hline Lsqft (000s) & $\begin{array}{c}0.001 \\
(1.316)\end{array}$ & $\begin{array}{c}0.001 \\
(1.344)\end{array}$ & $\begin{array}{c}0.001 \\
(1.315)\end{array}$ & $\begin{array}{c}0.001 \\
(1.321)\end{array}$ & $\begin{array}{c}0.001 \\
(1.321)\end{array}$ & $\begin{array}{c}0.001 \\
(1.305)\end{array}$ \\
\hline Hsqft (000s) & $\begin{array}{c}0.544 * * * \\
(13.474)\end{array}$ & $\begin{array}{c}0.544 * * * \\
(13.534)\end{array}$ & $\begin{array}{l}0.532 * * * \\
(13.035)\end{array}$ & $\begin{array}{c}0.545^{* * * *} \\
(13.503)\end{array}$ & $\begin{array}{c}0.546 * * * \\
(13.421)\end{array}$ & $\begin{array}{c}0.542 * * * \\
(13.412)\end{array}$ \\
\hline Hsqft2 (000s) & $\begin{array}{c}-0.029 * * * \\
(-9.587)\end{array}$ & $\begin{array}{c}-0.029 * * * \\
(-9.67)\end{array}$ & $\begin{array}{c}-0.028 * * * * \\
(-9.241)\end{array}$ & $\begin{array}{c}-0.028 * * * \\
(-9.583)\end{array}$ & $\begin{array}{c}-0.029 * * * \\
(-9.615)\end{array}$ & $\begin{array}{c}-0.028 * * * \\
(-9.6)\end{array}$ \\
\hline Age & $\begin{array}{c}0.004 \\
(1.038)\end{array}$ & $\begin{array}{c}0.004 \\
(1.077)\end{array}$ & $\begin{array}{c}0.003 \\
(0.947)\end{array}$ & $\begin{array}{c}0.004 \\
(1.111)\end{array}$ & $\begin{array}{c}0.004 \\
(1.073)\end{array}$ & $\begin{array}{c}0.004 \\
(1.088)\end{array}$ \\
\hline Age2 & $\begin{array}{c}0.000 \\
(-0.941)\end{array}$ & $\begin{array}{c}0.000 \\
(-0.968)\end{array}$ & $\begin{array}{c}0.000 \\
(-0.887)\end{array}$ & $\begin{array}{c}0.000 \\
(-0.994)\end{array}$ & $\begin{array}{c}0.000 \\
(-0.985)\end{array}$ & $\begin{array}{c}0.000 \\
(-1.022)\end{array}$ \\
\hline Bath & $\begin{array}{c}0.024 \\
(0.893)\end{array}$ & $\begin{array}{c}0.024 \\
(0.877)\end{array}$ & $\begin{array}{c}0.021 \\
(0.761)\end{array}$ & $\begin{array}{c}0.021 \\
(0.791)\end{array}$ & $\begin{array}{c}0.025 \\
(0.908)\end{array}$ & $\begin{array}{c}0.024 \\
(0.872)\end{array}$ \\
\hline Repeat & $\begin{array}{c}-0.083 * * \\
(-2.323)\end{array}$ & $\begin{array}{c}-0.084 * * \\
(-2.356)\end{array}$ & $\begin{array}{c}-0.083 * * \\
(-2.337)\end{array}$ & $\begin{array}{c}-0.084 * * \\
(-2.349)\end{array}$ & $\begin{array}{c}-0.082 * * \\
(-2.292)\end{array}$ & $\begin{array}{c}-0.084 * * \\
(-2.349)\end{array}$ \\
\hline \#rsdnc & $\begin{array}{c}-0.193 \\
(-1.341)\end{array}$ & $\begin{array}{c}-0.197 \\
(-1.358)\end{array}$ & $\begin{array}{c}-0.208 \\
(-1.455)\end{array}$ & $\begin{array}{c}-0.2 \\
(-1.394)\end{array}$ & $\begin{array}{c}-0.192 \\
(-1.331)\end{array}$ & $\begin{array}{c}-0.192 \\
(-1.332)\end{array}$ \\
\hline ExtrW & $\begin{array}{c}0.023 \\
(0.617)\end{array}$ & $\begin{array}{c}0.024 \\
(0.654)\end{array}$ & $\begin{array}{l}0.029 \\
(0.79)\end{array}$ & $\begin{array}{c}0.028 \\
(0.759)\end{array}$ & $\begin{array}{c}0.022 \\
(0.594)\end{array}$ & $\begin{array}{c}0.026 \\
(0.716)\end{array}$ \\
\hline Fire & $\begin{array}{c}0.034 \\
(0.764)\end{array}$ & $\begin{array}{c}0.029 \\
(0.657)\end{array}$ & $\begin{array}{c}0.03 \\
(0.684)\end{array}$ & $\begin{array}{c}0.029 \\
(0.635)\end{array}$ & $\begin{array}{c}0.033 \\
(0.751)\end{array}$ & $\begin{array}{c}0.032 \\
(0.724)\end{array}$ \\
\hline Enclsr & $\begin{array}{c}-0.196 * * * \\
(-4.149)\end{array}$ & $\begin{array}{c}-0.191 * * * \\
(-4.121)\end{array}$ & $\begin{array}{c}-0.187 * * * \\
(-4.073)\end{array}$ & $\begin{array}{c}-0.191 * * * \\
(-4.132)\end{array}$ & $\begin{array}{c}-0.196 * * * \\
(-4.14)\end{array}$ & $\begin{array}{c}-0.192 * * * \\
(-4.094)\end{array}$ \\
\hline Pool & $\begin{array}{c}0.125^{* * *} * \\
(3.087)\end{array}$ & $\begin{array}{c}0.123 * * * \\
(3.049)\end{array}$ & $\begin{array}{c}0.132 * * * \\
(3.297)\end{array}$ & $\begin{array}{c}0.126 * * * \\
(3.107)\end{array}$ & $\begin{array}{c}0.124 * * * \\
(3.067)\end{array}$ & $\begin{array}{c}0.123^{* * * *} \\
(3.044)\end{array}$ \\
\hline Blift & $\begin{array}{c}0.102 * * * \\
(2.66)\end{array}$ & $\begin{array}{c}0.105 * * * \\
(2.772)\end{array}$ & $\begin{array}{c}0.107 * * * \\
(2.818)\end{array}$ & $\begin{array}{c}0.107 * * * \\
(2.845)\end{array}$ & $\begin{array}{c}0.103 * * * \\
(2.675)\end{array}$ & $\begin{array}{c}0.104 * * * \\
(2.73)\end{array}$ \\
\hline Dock & $\begin{array}{c}0.115^{* * * *} \\
(2.599)\end{array}$ & $\begin{array}{c}0.115^{* * * *} \\
(2.605)\end{array}$ & $\begin{array}{c}0.117 * * * \\
(2.655)\end{array}$ & $\begin{array}{c}0.122 * * * \\
(2.735)\end{array}$ & $\begin{array}{c}0.115^{* * *} \\
(2.599)\end{array}$ & $\begin{array}{c}0.117 * * * \\
(2.643)\end{array}$ \\
\hline Special & $\begin{array}{c}0.05 \\
(1.088) \\
\end{array}$ & $\begin{array}{c}0.049 \\
(1.052) \\
\end{array}$ & $\begin{array}{c}0.041 \\
(0.898) \\
\end{array}$ & $\begin{array}{c}0.047 \\
(0.997) \\
\end{array}$ & $\begin{array}{c}0.051 \\
(1.096) \\
\end{array}$ & $\begin{array}{c}0.046 \\
(0.973) \\
\end{array}$ \\
\hline WQ & $\begin{array}{c}0.001 \\
(0.442)\end{array}$ & $\begin{array}{c}0.102 \\
(1.628)\end{array}$ & $\begin{array}{c}0.005 * * * \\
(2.67)\end{array}$ & $\begin{array}{c}0.004 \\
(1.264)\end{array}$ & $\begin{array}{c}-0.003 \\
(-0.268)\end{array}$ & $\begin{array}{c}0.001 \\
(1.152)\end{array}$ \\
\hline White & $\begin{array}{c}-0.001 \\
(-0.291)\end{array}$ & $\begin{array}{c}0.000 \\
(-0.093)\end{array}$ & $\begin{array}{c}0.000 \\
(-0.13)\end{array}$ & $\begin{array}{c}-0.001 \\
(-0.288)\end{array}$ & $\begin{array}{c}-0.001 \\
(-0.318)\end{array}$ & $\begin{array}{l}-0.001 \\
(-0.28)\end{array}$ \\
\hline 65 & $\begin{array}{c}-0.003 \\
(-1.312)\end{array}$ & $\begin{array}{c}-0.003 \\
(-1.303)\end{array}$ & $\begin{array}{c}-0.004 * \\
(-1.76)\end{array}$ & $\begin{array}{c}-0.003 \\
(-1.351)\end{array}$ & $\begin{array}{c}-0.003 \\
(-1.276)\end{array}$ & $\begin{array}{c}-0.003 \\
(-1.388)\end{array}$ \\
\hline Own & $\begin{array}{c}0.002 \\
(0.842)\end{array}$ & $\begin{array}{c}0.002 \\
(0.852)\end{array}$ & $\begin{array}{l}0.003 \\
(1.26)\end{array}$ & $\begin{array}{c}0.002 \\
(0.816)\end{array}$ & $\begin{array}{l}0.002 \\
(0.78)\end{array}$ & $\begin{array}{l}0.002 \\
(0.86)\end{array}$ \\
\hline Int & $\begin{array}{c}-0.157 * * * \\
(-8.186)\end{array}$ & $\begin{array}{c}-0.168 * * * \\
(-8.883)\end{array}$ & $\begin{array}{c}-0.174 * * * \\
(-8.764)\end{array}$ & $\begin{array}{c}-0.159 * * * \\
(-8.514)\end{array}$ & $\begin{array}{c}-0.155^{* * * *} \\
(-7.488)\end{array}$ & $\begin{array}{c}-0.161 * * * \\
(-8.239)\end{array}$ \\
\hline $\mathbf{R}^{2}$ & 0.56 & 0.56 & 0.56 & 0.56 & 0.56 & 0.56 \\
\hline$B P \sim 37.57$ & 44.85 & 44.91 & 48.36 & 46.33 & 47.85 & 52.06 \\
\hline
\end{tabular}

White's robust $t$-values are in parentheses

$*, * *$, and $* * *$ denote significance at the $0.10,0.05$ and 0.01 levels respectively 
Table III.7. Pooled OLS Results Median WQ

\begin{tabular}{|c|c|c|c|c|c|}
\hline$\underline{\text { Variable }}$ & WTemp & $\underline{\text { WVis }}$ & $\underline{\text { Salinit }}$ & $\underline{\text { DO }}$ & Grade \\
\hline intercept & $\begin{array}{c}11.879 * * * \\
(15.756)\end{array}$ & $\begin{array}{c}13.037 * * * \\
(37.831)\end{array}$ & $\begin{array}{c}12.953 * * * \\
(37.96)\end{array}$ & $\begin{array}{c}13.376^{* * *} \\
(31.172)\end{array}$ & $\begin{array}{c}13.311 * * * \\
(36.172)\end{array}$ \\
\hline Lsqft (000s) & $\begin{array}{c}0.001 \\
(1.333)\end{array}$ & $\begin{array}{c}0.001 \\
(1.312)\end{array}$ & $\begin{array}{c}0.000 \\
(1.217)\end{array}$ & $\begin{array}{c}0.001 \\
(1.356)\end{array}$ & $\begin{array}{c}0.001 \\
(1.287)\end{array}$ \\
\hline Hsqft (000s) & $\begin{array}{c}0.540 * * * \\
(13.513)\end{array}$ & $\begin{array}{c}0.545 * * * \\
(13.532)\end{array}$ & $\begin{array}{c}0.527 * * * \\
(12.971)\end{array}$ & $\begin{array}{c}0.547 * * * \\
(13.506)\end{array}$ & $\begin{array}{c}0.543 * * * \\
(13.607)\end{array}$ \\
\hline Hsqft2 (000s) & $\begin{array}{c}-0.028 * * * \\
(-9.655)\end{array}$ & $\begin{array}{c}-0.029 * * * \\
(-9.698)\end{array}$ & $\begin{array}{c}-0.027 * * * \\
(-9.219)\end{array}$ & $\begin{array}{c}-0.029 * * * \\
(-9.653)\end{array}$ & $\begin{array}{c}-0.029 * * * \\
(-9.712)\end{array}$ \\
\hline Age & $\begin{array}{c}0.005 \\
(1.096)\end{array}$ & $\begin{array}{c}0.004 \\
(1.091)\end{array}$ & $\begin{array}{c}0.003 \\
(0.831)\end{array}$ & $\begin{array}{c}0.004 \\
(1.116)\end{array}$ & $\begin{array}{c}0.004 \\
(1.003)\end{array}$ \\
\hline Age2 & $\begin{array}{c}0.000 \\
(-1.033)\end{array}$ & $\begin{array}{c}0.000 \\
(-1.007)\end{array}$ & $\begin{array}{c}0.000 \\
(-0.754)\end{array}$ & $\begin{array}{c}0.000 \\
(-1.003)\end{array}$ & $\begin{array}{c}0.000 \\
(-0.907)\end{array}$ \\
\hline Bath & $\begin{array}{c}0.027 \\
(0.967)\end{array}$ & $\begin{array}{c}0.024 \\
(0.894)\end{array}$ & $\begin{array}{c}0.021 \\
(0.771)\end{array}$ & $\begin{array}{c}0.024 \\
(0.884)\end{array}$ & $\begin{array}{c}0.027 \\
(1.008)\end{array}$ \\
\hline Repeat & $\begin{array}{c}-0.080 * * \\
(-2.236)\end{array}$ & $\begin{array}{c}-0.083 * * \\
(-2.315)\end{array}$ & $\begin{array}{c}-0.086 * * \\
(-2.447)\end{array}$ & $\begin{array}{c}-0.081 * * \\
(-2.265)\end{array}$ & $\begin{array}{c}-0.077 * * \\
(-2.15)\end{array}$ \\
\hline \#rsdnc & $\begin{array}{c}-0.200 \\
(-1.378)\end{array}$ & $\begin{array}{c}-0.196 \\
(-1.364)\end{array}$ & $\begin{array}{c}-0.215 \\
(-1.512)\end{array}$ & $\begin{array}{c}-0.201 \\
(-1.408)\end{array}$ & $\begin{array}{c}-0.186 \\
(-1.313)\end{array}$ \\
\hline ExtrW & $\begin{array}{c}0.026 \\
(0.697)\end{array}$ & $\begin{array}{c}0.025 \\
(0.674)\end{array}$ & $\begin{array}{c}0.037 \\
(1.000)\end{array}$ & $\begin{array}{c}0.021 \\
(0.572)\end{array}$ & $\begin{array}{c}0.015 \\
(0.413)\end{array}$ \\
\hline Fire & $\begin{array}{c}0.031 \\
(0.699)\end{array}$ & $\begin{array}{c}0.033 \\
(0.732)\end{array}$ & $\begin{array}{c}0.025 \\
(0.565)\end{array}$ & $\begin{array}{c}0.033 \\
(0.741)\end{array}$ & $\begin{array}{c}0.035 \\
(0.788)\end{array}$ \\
\hline Enclsr & $\begin{array}{c}-0.189 * * * \\
(-4.070)\end{array}$ & $\begin{array}{c}-0.193 * * * \\
(-4.163)\end{array}$ & $\begin{array}{c}-0.174 * * * \\
(-3.826)\end{array}$ & $\begin{array}{c}-0.196 * * * \\
(-4.164)\end{array}$ & $\begin{array}{c}-0.194 * * * \\
(-4.087)\end{array}$ \\
\hline Pool & $\begin{array}{c}0.126^{* * *} \\
(3.137)\end{array}$ & $\begin{array}{c}0.125^{* * * *} \\
(3.079)\end{array}$ & $\begin{array}{c}0.129 * * * \\
(3.24)\end{array}$ & $\begin{array}{c}0.124 * * * \\
(3.063)\end{array}$ & $\begin{array}{l}0.12 * * * \\
(2.969)\end{array}$ \\
\hline Blift & $\begin{array}{c}0.103^{* * *} \\
(2.691)\end{array}$ & $\begin{array}{c}0.104 * * * \\
(2.753)\end{array}$ & $\begin{array}{c}0.112 * * * \\
(2.988)\end{array}$ & $\begin{array}{c}0.102 * * * \\
(2.675)\end{array}$ & $\begin{array}{c}0.097 * * \\
(2.535)\end{array}$ \\
\hline Dock & $\begin{array}{c}0.117 * * * \\
(2.649)\end{array}$ & $\begin{array}{c}0.118 * * * \\
(2.628)\end{array}$ & $\begin{array}{c}0.134 * * * \\
(3.041)\end{array}$ & $\begin{array}{l}0.12 * * * \\
(2.692)\end{array}$ & $\begin{array}{l}0.107 * * \\
(2.364)\end{array}$ \\
\hline Special & $\begin{array}{c}0.045 \\
(0.973)\end{array}$ & $\begin{array}{l}0.048 \\
(1.01)\end{array}$ & $\begin{array}{c}0.04 \\
(0.874)\end{array}$ & $\begin{array}{c}0.052 \\
(1.114)\end{array}$ & $\begin{array}{c}0.052 \\
(1.127)\end{array}$ \\
\hline WQ & $\begin{array}{l}0.013^{*} \\
(1.911) \\
\end{array}$ & $\begin{array}{c}0 \\
(0.535) \\
\end{array}$ & $\begin{array}{c}0.009^{* * * *} \\
(3.613) \\
\end{array}$ & $\begin{array}{c}-0.041 \\
(-1.052) \\
\end{array}$ & $\begin{array}{l}-0.003^{*} \\
(-1.892) \\
\end{array}$ \\
\hline White & $\begin{array}{c}-0.001 \\
(-0.170)\end{array}$ & $\begin{array}{c}-0.001 \\
(-0.332)\end{array}$ & $\begin{array}{c}0.000 \\
(0.153)\end{array}$ & $\begin{array}{c}-0.001 \\
(-0.395)\end{array}$ & $\begin{array}{c}0.000 \\
(-0.219)\end{array}$ \\
\hline 65 & $\begin{array}{c}-0.004 \\
(-1.643)\end{array}$ & $\begin{array}{c}-0.003 \\
(-1.398)\end{array}$ & $\begin{array}{l}-0.004 * \\
(-1.859)\end{array}$ & $\begin{array}{l}-0.003 \\
(-1.276)\end{array}$ & $\begin{array}{l}-0.002 \\
(-1.095)\end{array}$ \\
\hline Own & $\begin{array}{c}0.003 \\
(1.138)\end{array}$ & $\begin{array}{c}0.002 \\
(0.887)\end{array}$ & $\begin{array}{c}0.002 \\
(0.969)\end{array}$ & $\begin{array}{c}0.002 \\
(0.715)\end{array}$ & $\begin{array}{c}0.002 \\
(0.789)\end{array}$ \\
\hline Int & $\begin{array}{c}-0.143 * * * \\
(-7.110)\end{array}$ & $\begin{array}{c}-0.159 * * * \\
(-8.173)\end{array}$ & $\begin{array}{c}-0.178 * * * \\
(-9.276)\end{array}$ & $\begin{array}{c}-0.159 * * * \\
(-8.414)\end{array}$ & $\begin{array}{c}-0.151 * * * \\
(-7.887)\end{array}$ \\
\hline $\mathbf{R}^{2}$ & 0.56 & 0.56 & 0.57 & 0.56 & 0.56 \\
\hline BP $\sim 37.57$ & 46.99 & 49.36 & 48.33 & 44.14 & 44.87 \\
\hline
\end{tabular}

White's robust $t$-values are in parentheses

$*, * *$, and *** denote significance at the $0.10,0.05$ and 0.01 levels respectively

Results for $\mathrm{pH}$ are excluded due to multicollinearity issues between median $\mathrm{pH}$ values and the constant 
Table III.8. Pooled OLS Results Statistical Range of WQ

\begin{tabular}{|c|c|c|c|c|c|c|}
\hline Variable & WTemp & $\underline{\mathbf{H}}$ & $\underline{\text { WVis }}$ & $\underline{\text { Salinit }}$ & $\underline{\text { DO }}$ & $\underline{\text { Grade }}$ \\
\hline intercept & $\begin{array}{c}13.015 * * * \\
(35.716)\end{array}$ & $\begin{array}{c}13.075 * * * \\
(38.859)\end{array}$ & $\begin{array}{c}13.199 * * * \\
(38.368)\end{array}$ & $\begin{array}{c}12.921 * * * \\
(36.807)\end{array}$ & $\begin{array}{c}13.155^{* * * *} \\
(36.666)\end{array}$ & $\begin{array}{c}13.117 * * * \\
(37.435)\end{array}$ \\
\hline Lsqft (000s) & $\begin{array}{c}0.001 \\
(1.314)\end{array}$ & $\begin{array}{c}0.001 \\
(1.306)\end{array}$ & $\begin{array}{c}0.001 \\
(1.326)\end{array}$ & $\begin{array}{c}0.001 \\
(1.253)\end{array}$ & $\begin{array}{c}0.001 \\
(1.305)\end{array}$ & $\begin{array}{c}0.001 \\
(1.316)\end{array}$ \\
\hline Hsqft (000s) & $\begin{array}{c}0.545^{* * * *} \\
(13.51)\end{array}$ & $\begin{array}{c}0.545^{* * * *} \\
(13.536)\end{array}$ & $\begin{array}{c}0.543 * * * \\
(13.46)\end{array}$ & $\begin{array}{c}0.538 * * * \\
(13.102)\end{array}$ & $\begin{array}{l}0.54 * * * \\
(13.384)\end{array}$ & $\begin{array}{c}0.543 * * * \\
(13.453)\end{array}$ \\
\hline Hsqft2 (000s) & $\begin{array}{c}-0.029 * * * \\
(-9.639)\end{array}$ & $\begin{array}{c}-0.029 * * * \\
(-9.675)\end{array}$ & $\begin{array}{c}-0.028 * * * \\
(-9.523)\end{array}$ & $\begin{array}{c}-0.029 * * * \\
(-9.676)\end{array}$ & $\begin{array}{c}-0.028 * * * \\
(-9.455)\end{array}$ & $\begin{array}{c}-0.029 * * * \\
(-9.627)\end{array}$ \\
\hline Age & $\begin{array}{c}0.004 \\
(1.091)\end{array}$ & $\begin{array}{c}0.004 \\
(1.048)\end{array}$ & $\begin{array}{c}0.004 \\
(1.001)\end{array}$ & $\begin{array}{c}0.003 \\
(0.892)\end{array}$ & $\begin{array}{l}0.003 \\
(0.95)\end{array}$ & $\begin{array}{c}0.004 \\
(1.079)\end{array}$ \\
\hline Age2 & $\begin{array}{c}0.000 \\
(-1.005)\end{array}$ & $\begin{array}{c}0.000 \\
(-0.942)\end{array}$ & $\begin{array}{c}0.000 \\
(-0.891)\end{array}$ & $\begin{array}{c}0.000 \\
(-0.859)\end{array}$ & $\begin{array}{c}0.000 \\
(-0.867)\end{array}$ & $\begin{array}{c}0.000 \\
(-1)\end{array}$ \\
\hline Bath & $\begin{array}{c}0.025 \\
(0.923)\end{array}$ & $\begin{array}{c}0.024 \\
(0.896)\end{array}$ & $\begin{array}{c}0.022 \\
(0.813)\end{array}$ & $\begin{array}{c}0.027 \\
(0.989)\end{array}$ & $\begin{array}{c}0.025 \\
(0.905)\end{array}$ & $\begin{array}{c}0.024 \\
(0.885)\end{array}$ \\
\hline Repeat & $\begin{array}{c}-0.082 * * \\
(-2.296)\end{array}$ & $\begin{array}{c}-0.083 * * \\
(-2.327)\end{array}$ & $\begin{array}{l}-0.085 * * \\
(-2.373)\end{array}$ & $\begin{array}{l}-0.083 * * \\
(-2.324)\end{array}$ & $\begin{array}{c}-0.084 * * \\
(-2.353)\end{array}$ & $\begin{array}{l}-0.084 * * \\
(-2.337)\end{array}$ \\
\hline \#rsdnc & $\begin{array}{c}-0.191 \\
(-1.324)\end{array}$ & $\begin{array}{l}-0.193 \\
(-1.34)\end{array}$ & $\begin{array}{c}-0.193 \\
(-1.347)\end{array}$ & $\begin{array}{l}-0.192 \\
(-1.33)\end{array}$ & $\begin{array}{c}-0.193 \\
(-1.343)\end{array}$ & $\begin{array}{c}-0.192 \\
(-1.334)\end{array}$ \\
\hline ExtrW & $\begin{array}{c}0.023 \\
(0.616)\end{array}$ & $\begin{array}{c}0.024 \\
(0.648)\end{array}$ & $\begin{array}{c}0.022 \\
(0.584)\end{array}$ & $\begin{array}{c}0.021 \\
(0.583)\end{array}$ & $\begin{array}{c}0.022 \\
(0.599)\end{array}$ & $\begin{array}{c}0.024 \\
(0.648)\end{array}$ \\
\hline Fire & $\begin{array}{c}0.033 \\
(0.755)\end{array}$ & $\begin{array}{l}0.032 \\
(0.71)\end{array}$ & $\begin{array}{c}0.033 \\
(0.751)\end{array}$ & $\begin{array}{l}0.037 \\
(0.82)\end{array}$ & $\begin{array}{c}0.035 \\
(0.793)\end{array}$ & $\begin{array}{c}0.033 \\
(0.747)\end{array}$ \\
\hline Enclsr & $\begin{array}{c}-0.195 * * * \\
(-4.143)\end{array}$ & $\begin{array}{c}-0.193 * * * \\
(-4.135)\end{array}$ & $\begin{array}{c}-0.198 * * * \\
(-4.193)\end{array}$ & $\begin{array}{c}-0.193 * * * \\
(-4.055)\end{array}$ & $\begin{array}{c}-0.194 * * * \\
(-4.08)\end{array}$ & $\begin{array}{c}-0.194 * * * \\
(-4.129)\end{array}$ \\
\hline Pool & $\begin{array}{c}0.124 * * * \\
(3.068)\end{array}$ & $\begin{array}{c}0.123 * * * \\
(3.046)\end{array}$ & $\begin{array}{c}0.126^{* * * *} \\
(3.117)\end{array}$ & $\begin{array}{c}0.123^{* * * *} \\
(3.044)\end{array}$ & $\begin{array}{c}0.123 * * * \\
(3.019)\end{array}$ & $\begin{array}{c}0.123 * * * \\
(3.048)\end{array}$ \\
\hline Blift & $\begin{array}{c}0.103^{* * * *} \\
(2.68)\end{array}$ & $\begin{array}{c}0.104 * * * \\
(2.72)\end{array}$ & $\begin{array}{c}0.104 * * * \\
(2.697)\end{array}$ & $\begin{array}{l}0.1 * * * \\
(2.623)\end{array}$ & $\begin{array}{c}0.102 * * * \\
(2.65)\end{array}$ & $\begin{array}{c}0.104 * * * \\
(2.703)\end{array}$ \\
\hline Dock & $\begin{array}{c}0.115^{* * * *} \\
(2.6)\end{array}$ & $\begin{array}{c}0.116^{* * *} \\
(2.618)\end{array}$ & $\begin{array}{c}0.115 * * * \\
(2.582)\end{array}$ & $\begin{array}{c}0.114 * * \\
(2.566)\end{array}$ & $\begin{array}{c}0.117 * * * \\
(2.638)\end{array}$ & $\begin{array}{c}0.117 * * * \\
(2.628)\end{array}$ \\
\hline Special & $\begin{array}{c}0.05 \\
(1.072) \\
\end{array}$ & $\begin{array}{c}0.049 \\
(1.039) \\
\end{array}$ & $\begin{array}{c}0.052 \\
(1.128) \\
\end{array}$ & $\begin{array}{c}0.05 \\
(1.08) \\
\end{array}$ & $\begin{array}{c}0.051 \\
(1.099) \\
\end{array}$ & $\begin{array}{c}0.049 \\
(1.044) \\
\end{array}$ \\
\hline WQ & $\begin{array}{c}0.001 \\
(0.388) \\
\end{array}$ & $\begin{array}{l}-0.046 \\
(-0.76) \\
\end{array}$ & $\begin{array}{c}-0.002 \\
(-1.278) \\
\end{array}$ & $\begin{array}{c}0.004 \\
(1.153) \\
\end{array}$ & $\begin{array}{c}-0.009 \\
(-0.987) \\
\end{array}$ & $\begin{array}{c}0 \\
(-0.638) \\
\end{array}$ \\
\hline White & $\begin{array}{c}-0.001 \\
(-0.298)\end{array}$ & $\begin{array}{c}0.000 \\
(-0.227)\end{array}$ & $\begin{array}{c}0.000 \\
(-0.248)\end{array}$ & $\begin{array}{c}0.000 \\
(-0.199)\end{array}$ & $\begin{array}{c}0.000 \\
(-0.272)\end{array}$ & $\begin{array}{c}-0.001 \\
(-0.299)\end{array}$ \\
\hline 65 & $\begin{array}{c}-0.003 \\
(-1.328)\end{array}$ & $\begin{array}{c}-0.003 \\
(-1.324)\end{array}$ & $\begin{array}{l}-0.003 \\
(-1.19)\end{array}$ & $\begin{array}{c}-0.004 \\
(-1.565)\end{array}$ & $\begin{array}{c}-0.003 \\
(-1.387)\end{array}$ & $\begin{array}{c}-0.003 \\
(-1.326)\end{array}$ \\
\hline Own & $\begin{array}{c}0.002 \\
(0.768)\end{array}$ & $\begin{array}{c}0.002 \\
(0.853)\end{array}$ & $\begin{array}{c}0.002 \\
(0.734)\end{array}$ & $\begin{array}{c}0.003 \\
(1.029)\end{array}$ & $\begin{array}{c}0.002 \\
(0.825)\end{array}$ & $\begin{array}{l}0.002 \\
(0.81)\end{array}$ \\
\hline Int & $\begin{array}{c}-0.158 * * * \\
(-8.333)\end{array}$ & $\begin{array}{c}-0.156 * * * \\
(-8.241)\end{array}$ & $\begin{array}{c}-0.157 * * * \\
(-8.273)\end{array}$ & $\begin{array}{c}-0.157 * * * \\
(-8.28)\end{array}$ & $\begin{array}{c}-0.161 * * * \\
(-7.934)\end{array}$ & $\begin{array}{c}-0.158 * * * \\
(-8.255)\end{array}$ \\
\hline $\begin{array}{l}\mathbf{R}^{2} \\
\mathbf{B P} \sim 37.57\end{array}$ & $\begin{array}{c}0.56 \\
44.44\end{array}$ & $\begin{array}{c}0.56 \\
49.07\end{array}$ & $\begin{array}{c}0.56 \\
44.51\end{array}$ & $\begin{array}{c}0.56 \\
46.80\end{array}$ & $\begin{array}{c}0.56 \\
48.03\end{array}$ & $\begin{array}{c}0.56 \\
50.82\end{array}$ \\
\hline
\end{tabular}

White's robust $t$-values are in parentheses

$*$,**, and $* * *$ denote significance at the $0.10,0.05$ and 0.01 levels respectively 
Table III.9. Pooled OLS Results Maximum WQ

\begin{tabular}{|c|c|c|c|c|c|c|}
\hline Variable & WTemp & pH & $\underline{\text { WVis }}$ & $\underline{\text { Salinit }}$ & $\underline{\text { DO }}$ & Grade \\
\hline intercept & $\begin{array}{c}11.851 * * * \\
(15.248)\end{array}$ & $\begin{array}{c}12.137 * * * \\
(14.472)\end{array}$ & $\begin{array}{c}12.786 * * * \\
(34.952)\end{array}$ & $\begin{array}{c}12.789 * * * \\
(35.791)\end{array}$ & $\begin{array}{c}13.465 * * * \\
(34.901)\end{array}$ & $\begin{array}{c}12.443 * * * \\
(25.489)\end{array}$ \\
\hline Lsqft (000s) & $\begin{array}{c}0.001 \\
(1.288)\end{array}$ & $\begin{array}{c}0.001 \\
(1.374)\end{array}$ & $\begin{array}{c}0.001 \\
(1.301)\end{array}$ & $\begin{array}{c}0.001 \\
(1.202)\end{array}$ & $\begin{array}{c}0.001 \\
(1.332)\end{array}$ & $\begin{array}{c}0.001 \\
(1.274)\end{array}$ \\
\hline Hsqft (000s) & $\begin{array}{l}0.543 * * * \\
(13.476)\end{array}$ & $\begin{array}{c}0.543^{* * *} * \\
(13.521)\end{array}$ & $\begin{array}{l}0.54 * * * \\
(13.436)\end{array}$ & $\begin{array}{c}0.533 * * * \\
(13.085)\end{array}$ & $\begin{array}{l}0.54 * * * \\
(13.503)\end{array}$ & $\begin{array}{c}0.544 * * * \\
(13.533)\end{array}$ \\
\hline Hsqft2 (000s) & $\begin{array}{c}-0.029 * * * \\
(-9.775)\end{array}$ & $\begin{array}{c}-0.029 * * * \\
(-9.658)\end{array}$ & $\begin{array}{c}-0.029 * * * \\
(-9.678)\end{array}$ & $\begin{array}{c}-0.028 * * * \\
(-9.41)\end{array}$ & $\begin{array}{c}-0.028 * * * \\
(-9.642)\end{array}$ & $\begin{array}{c}-0.029 * * * \\
(-9.732)\end{array}$ \\
\hline Age & $\begin{array}{c}0.004 \\
(1.056)\end{array}$ & $\begin{array}{c}0.004 \\
(1.116)\end{array}$ & $\begin{array}{c}0.004 \\
(1.106)\end{array}$ & $\begin{array}{c}0.003 \\
(0.839)\end{array}$ & $\begin{array}{l}0.003 \\
(0.91)\end{array}$ & $\begin{array}{c}0.004 \\
(1.057)\end{array}$ \\
\hline Age2 & $\begin{array}{c}0.000 \\
(-0.986)\end{array}$ & $\begin{array}{c}0.000 \\
(-1.034)\end{array}$ & $\begin{array}{c}0.000 \\
(-1.064)\end{array}$ & $\begin{array}{c}0.000 \\
(-0.79)\end{array}$ & $\begin{array}{c}0.000 \\
(-0.812)\end{array}$ & $\begin{array}{c}0.000 \\
(-0.976)\end{array}$ \\
\hline Bath & $\begin{array}{c}0.025 \\
(0.921)\end{array}$ & $\begin{array}{c}0.025 \\
(0.901)\end{array}$ & $\begin{array}{c}0.026 \\
(0.961)\end{array}$ & $\begin{array}{c}0.022 \\
(0.828)\end{array}$ & $\begin{array}{c}0.025 \\
(0.917)\end{array}$ & $\begin{array}{l}0.026 \\
(0.94)\end{array}$ \\
\hline Repeat & $\begin{array}{l}-0.082 * * \\
(-2.292)\end{array}$ & $\begin{array}{c}-0.083 * * \\
(-2.323)\end{array}$ & $\begin{array}{l}-0.08 * * \\
(-2.239)\end{array}$ & $\begin{array}{c}-0.086 * * \\
(-2.413)\end{array}$ & $\begin{array}{c}-0.084 * * \\
(-2.352)\end{array}$ & $\begin{array}{c}-0.079 * * \\
(-2.213)\end{array}$ \\
\hline \#rsdnc & $\begin{array}{l}-0.191 \\
(-1.32)\end{array}$ & $\begin{array}{c}-0.195 \\
(-1.343)\end{array}$ & $\begin{array}{c}-0.2 \\
(-1.388)\end{array}$ & $\begin{array}{c}-0.209 \\
(-1.449)\end{array}$ & $\begin{array}{c}-0.194 \\
(-1.352)\end{array}$ & $\begin{array}{c}-0.191 \\
(-1.325)\end{array}$ \\
\hline ExtrW & $\begin{array}{c}0.031 \\
(0.833)\end{array}$ & $\begin{array}{c}0.02 \\
(0.53)\end{array}$ & $\begin{array}{l}0.028 \\
(0.75)\end{array}$ & $\begin{array}{c}0.033 \\
(0.889)\end{array}$ & $\begin{array}{l}0.018 \\
(0.48)\end{array}$ & $\begin{array}{c}0.027 \\
(0.724)\end{array}$ \\
\hline Fire & $\begin{array}{c}0.034 \\
(0.758)\end{array}$ & $\begin{array}{c}0.034 \\
(0.763)\end{array}$ & $\begin{array}{c}0.032 \\
(0.729)\end{array}$ & $\begin{array}{c}0.03 \\
(0.672)\end{array}$ & $\begin{array}{c}0.036 \\
(0.819)\end{array}$ & $\begin{array}{c}0.032 \\
(0.715)\end{array}$ \\
\hline Enclsr & $\begin{array}{c}-0.192 * * * \\
(-4.077)\end{array}$ & $\begin{array}{c}-0.196^{* * *} \\
(-4.169)\end{array}$ & $\begin{array}{c}-0.187 * * * \\
(-4.049)\end{array}$ & $\begin{array}{c}-0.181 * * * \\
(-3.898)\end{array}$ & $\begin{array}{c}-0.194 * * * \\
(-4.113)\end{array}$ & $\begin{array}{c}-0.194 * * * \\
(-4.129)\end{array}$ \\
\hline Pool & $\begin{array}{c}0.129 * * * \\
(3.207)\end{array}$ & $\begin{array}{c}0.126 * * * \\
(3.094)\end{array}$ & $\begin{array}{c}0.125 * * * \\
(3.123)\end{array}$ & $\begin{array}{c}0.126^{* * * *} \\
(3.153)\end{array}$ & $\begin{array}{c}0.118 * * * \\
(2.914)\end{array}$ & $\begin{array}{c}0.125 * * * \\
(3.118)\end{array}$ \\
\hline Blift & $\begin{array}{c}0.104 * * * \\
(2.705)\end{array}$ & $\begin{array}{c}0.102 * * * \\
(2.671)\end{array}$ & $\begin{array}{c}0.103 * * * \\
(2.696)\end{array}$ & $\begin{array}{c}0.108 * * * \\
(2.845)\end{array}$ & $\begin{array}{c}0.102 * * * \\
(2.656)\end{array}$ & $\begin{array}{c}0.101 * * * \\
(2.636)\end{array}$ \\
\hline Dock & $\begin{array}{c}0.116^{* * * *} \\
(2.6)\end{array}$ & $\begin{array}{c}0.114 * * * \\
(2.587)\end{array}$ & $\begin{array}{c}0.118^{* * * *} \\
(2.662)\end{array}$ & $\begin{array}{c}0.125 * * * \\
(2.826)\end{array}$ & $\begin{array}{c}0.121 * * * \\
(2.731)\end{array}$ & $\begin{array}{c}0.113 * * * \\
(2.535)\end{array}$ \\
\hline Special & $\begin{array}{c}0.044 \\
(0.947) \\
\end{array}$ & $\begin{array}{c}0.054 \\
(1.146) \\
\end{array}$ & $\begin{array}{c}0.042 \\
(0.897) \\
\end{array}$ & $\begin{array}{c}0.042 \\
(0.895) \\
\end{array}$ & $\begin{array}{c}0.054 \\
(1.175) \\
\end{array}$ & $\begin{array}{c}0.045 \\
(0.967) \\
\end{array}$ \\
\hline WQ & $\begin{array}{c}0.014 * \\
(1.73) \\
\end{array}$ & $\begin{array}{c}0.122 \\
(1.273) \\
\end{array}$ & $\begin{array}{c}0.003 * * \\
(1.994) \\
\end{array}$ & $\begin{array}{c}0.008 * * \\
(2.543)\end{array}$ & $\begin{array}{c}-0.035 * * \\
(-2.047)\end{array}$ & $\begin{array}{c}0.006^{*} \\
(1.709) \\
\end{array}$ \\
\hline White & $\begin{array}{c}0.000 \\
(-0.127)\end{array}$ & $\begin{array}{c}0.000 \\
(-0.255)\end{array}$ & $\begin{array}{l}-0.001 \\
(-0.29)\end{array}$ & $\begin{array}{c}0.000 \\
(-0.076)\end{array}$ & $\begin{array}{c}-0.001 \\
(-0.323)\end{array}$ & $\begin{array}{c}0.000 \\
(-0.254)\end{array}$ \\
\hline 65 & $\begin{array}{c}-0.003 \\
(-1.456)\end{array}$ & $\begin{array}{c}-0.003 \\
(-1.249)\end{array}$ & $\begin{array}{c}-0.004 * \\
(-1.73)\end{array}$ & $\begin{array}{c}-0.005^{*} \\
(-1.94)\end{array}$ & $\begin{array}{c}-0.003 \\
(-1.413)\end{array}$ & $\begin{array}{c}-0.003 \\
(-1.506)\end{array}$ \\
\hline Own & $\begin{array}{c}0.002 \\
(0.938)\end{array}$ & $\begin{array}{c}0.002 \\
(0.694)\end{array}$ & $\begin{array}{c}0.003 \\
(1.167)\end{array}$ & $\begin{array}{l}0.003 \\
(1.24)\end{array}$ & $\begin{array}{c}0.002 \\
(0.699)\end{array}$ & $\begin{array}{c}0.002 \\
(0.958)\end{array}$ \\
\hline Int & $\begin{array}{c}-0.171 * * * \\
(-7.964)\end{array}$ & $\begin{array}{c}-0.171 * * * \\
(-8.316)\end{array}$ & $\begin{array}{c}-0.168 * * * \\
(-8.368)\end{array}$ & $\begin{array}{c}-0.162 * * * \\
(-8.576)\end{array}$ & $\begin{array}{c}-0.158 * * * \\
(-8.331)\end{array}$ & $\begin{array}{c}-0.165 * * * \\
(-8.367)\end{array}$ \\
\hline $\begin{array}{l}\mathbf{R}^{2} \\
\mathbf{B P} \sim 37.57\end{array}$ & $\begin{array}{c}0.56 \\
47.63\end{array}$ & $\begin{array}{c}0.56 \\
50.71\end{array}$ & $\begin{array}{c}0.56 \\
46.53\end{array}$ & $\begin{array}{c}0.56 \\
53.66\end{array}$ & $\begin{array}{c}0.56 \\
44.88\end{array}$ & $\begin{array}{c}0.56 \\
44.52\end{array}$ \\
\hline
\end{tabular}

White's robust $t$-values are in parentheses

$*, * *$, and *** denote significance at the $0.10,0.05$ and 0.01 levels respectively 
Table III.10. Pooled WQ Measure Results by Statistical Descriptor

\begin{tabular}{|c|c|c|c|c|}
\cline { 2 - 5 } \multicolumn{1}{c|}{} & \multicolumn{4}{c|}{ WQ Measure For Year of Sale } \\
\cline { 2 - 5 } & Minimum & Median & Range & Maximum \\
\hline WTemp & & $*$ & & $*$ \\
\hline WH & & & & \\
\hline Salinity & $* * *$ & & & $* *$ \\
\hline DO & & $* * *$ & & $* *$ \\
\hline Grade & & & & $* *$ \\
\hline
\end{tabular}

$*$, **, and *** denote significance at the $0.10,0.05$ and 0.01 levels respectively

Table III.11. Pooled WQ Measure Hedonic Prices by Statistical Descriptor

\begin{tabular}{|c|c|c|c|c|}
\cline { 2 - 5 } \multicolumn{1}{c|}{} & \multicolumn{3}{|c|}{ WQ Measure For Year of Sale } \\
\cline { 2 - 5 } & Minimum & Median & Range & Maximum \\
\hline WTemp & & $\begin{array}{c}\$ 11,284 \\
(1.3 \%)\end{array}$ & & $\begin{array}{c}\$ 12,152 \\
(1.4 \%)\end{array}$ \\
\hline pH & & & & \\
\hline WVis & $\$ 4,340$ & & & $\begin{array}{c}\$ 2,604 \\
(0.3 \%)\end{array}$ \\
\hline Salinit & $(0.5 \%)$ & & $\begin{array}{c}\$ 6,944 \\
(0.8 \%)\end{array}$ \\
\hline DO & & $(0.9 \%)$ & & $\begin{array}{c}\$-30,380 \\
(3.5 \%)\end{array}$ \\
\hline Grade & & & & $\begin{array}{c}\$ 5,208 \\
(0.6 \%)\end{array}$ \\
\hline
\end{tabular}

The $\%$ change in the mean sales price for an additional unit of each variable is given in parentheses, i.e., the estimated coefficient for the respective WQ measure as per Tables 6 to 9. 
Table III.12. Fixed Effect OLS Results Minimum WQ

\begin{tabular}{lcccccc} 
Variable & WTemp & pH & WVis & Salinit & $\underline{\text { DO }}$ & Grade \\
\hline WQ & -0.004 & $-0.219^{* *}$ & -0.003 & $-0.025^{* * *}$ & 0.005 & $-0.005^{* * *}$ \\
& $(-0.779)$ & $(-2.387)$ & $(-1.211)$ & $(-4.043)$ & $(0.335)$ & $(-2.993)$ \\
\hline d_river & $-0.211^{* * *}$ & $-0.268^{* * *}$ & $-0.284^{* * *}$ & $-0.652^{* * *}$ & $-0.224 * * *$ & $-0.308^{* * *}$ \\
& $(-3.079)$ & $(-4.03)$ & $(-3.417)$ & $(-5.576)$ & $(-3.28)$ & $(-4.203)$ \\
d_estry & $0.186^{* *}$ & $0.188^{* *}$ & $0.144^{*}$ & -0.16 & $0.155^{*}$ & 0.127 \\
& $(2.049)$ & $(2.221)$ & $(1.667)$ & $(-1.468)$ & $(1.786)$ & $(1.499)$ \\
d_2000 & 0.029 & 0.164 & 0.068 & 0.137 & 0.013 & 0.089 \\
& $(0.203)$ & $(1.046)$ & $(0.451)$ & $(0.942)$ & $(0.088)$ & $(0.604)$ \\
d_2001 & -0.071 & -0.004 & -0.051 & -0.057 & -0.077 & -0.067 \\
& $(-0.791)$ & $(-0.049)$ & $(-0.559)$ & $(-0.64)$ & $(-0.819)$ & $(-0.751)$ \\
d_2002 & -0.064 & 0.018 & -0.037 & -0.067 & -0.089 & -0.03 \\
& $(-0.994)$ & $(0.235)$ & $(-0.539)$ & $(-1.092)$ & $(-1.268)$ & $(-0.483)$ \\
d_2003 & -0.049 & 0.058 & -0.012 & -0.008 & -0.027 & -0.016 \\
& $(-0.845)$ & $(0.922)$ & $(-0.25)$ & $(-0.168)$ & $(-0.552)$ & $(-0.34)$
\end{tabular}

White's robust $t$-values are in parentheses

$*$, **, and *** denote significance at the $0.10,0.05$ and 0.01 levels respectively

Table III.13. Fixed Effect OLS Results Median WQ

\begin{tabular}{|c|c|c|c|c|c|c|}
\hline Variable & WTemp & $\underline{\mathbf{p H}}$ & WVis & $\underline{\text { Salinit }}$ & $\underline{\text { DO }}$ & Grade \\
\hline WQ & -0.015 & & $-0.004 * * *$ & -0.003 & $-0.132 * * *$ & $-0.011 * * *$ \\
\hline & $(-1.359)$ & & $(-3.014)$ & $(-0.522)$ & $(-2.732)$ & $(-3.657)$ \\
\hline d_river & $\begin{array}{c}-0.237 * * * \\
(-3.486)\end{array}$ & & $\begin{array}{c}-0.419 * * * \\
(-4.323)\end{array}$ & $\begin{array}{l}-0.292^{*} \\
(-1.814)\end{array}$ & $\begin{array}{c}-0.157 * * \\
(-2.252)\end{array}$ & $\begin{array}{c}-0.375^{* * *} \\
(-4.716)\end{array}$ \\
\hline d_estry & $\begin{array}{l}0.187 * * \\
(2.157)\end{array}$ & & $\begin{array}{l}0.008 \\
(0.08)\end{array}$ & $\begin{array}{c}0.131 \\
(1.187)\end{array}$ & $\begin{array}{c}0.266 * * * \\
(2.819)\end{array}$ & $\begin{array}{c}0.003 \\
(0.037)\end{array}$ \\
\hline d_2000 & $\begin{array}{c}0.037 \\
(0.258)\end{array}$ & & $\begin{array}{c}0.087 \\
(0.603)\end{array}$ & $\begin{array}{c}0.043 \\
(0.285)\end{array}$ & $\begin{array}{c}-0.061 \\
(-0.407)\end{array}$ & $\begin{array}{c}0.004 \\
(0.027)\end{array}$ \\
\hline d_2001 & $\begin{array}{c}-0.056 \\
(-0.623)\end{array}$ & & $\begin{array}{c}-0.045 \\
(-0.501)\end{array}$ & $\begin{array}{c}-0.04 \\
(-0.363)\end{array}$ & $\begin{array}{c}-0.124 \\
(-1.296)\end{array}$ & $\begin{array}{c}-0.17 * \\
(-1.704)\end{array}$ \\
\hline d_2002 & $\begin{array}{c}-0.006 \\
(-0.082)\end{array}$ & & $\begin{array}{c}-0.048 \\
(-0.799)\end{array}$ & $\begin{array}{c}-0.062 \\
(-0.895)\end{array}$ & $\begin{array}{c}-0.184 * * \\
(-2.484)\end{array}$ & $\begin{array}{l}-0.049 \\
(-0.8)\end{array}$ \\
\hline d_2003 & $\begin{array}{c}0.032 \\
(0.484)\end{array}$ & & $\begin{array}{c}-0.034 \\
(-0.695)\end{array}$ & $\begin{array}{c}-0.038 \\
(-0.755)\end{array}$ & $\begin{array}{l}-0.128 * * \\
(-1.995)\end{array}$ & $\begin{array}{c}-0.169 * * * \\
(-2.831)\end{array}$ \\
\hline
\end{tabular}

White's robust $t$-values are in parentheses

$*, * *$, and $* * *$ denote significance at the $0.10,0.05$ and 0.01 levels respectively

Results for $\mathrm{pH}$ are excluded due to multicollinearity issues between median $\mathrm{pH}$ values and the constant 
Table III.14. Fixed Effect OLS Results Statistical Range of WQ

\begin{tabular}{lcccccc} 
Variable & WTemp & pH & WVis & Salinit & $\underline{\text { DO }}$ & Grade \\
\hline WQ & -0.002 & $0.197^{* *}$ & 0.001 & $0.018^{* * *}$ & $-0.023^{* *}$ & $0.004^{* * *}$ \\
& $(-0.485)$ & $(2.567)$ & $(0.591)$ & $(2.763)$ & $(-1.987)$ & $(2.916)$ \\
\hline d_river & $-0.228^{* * *}$ & $-0.269^{* * *}$ & $-0.226^{* * *}$ & $-0.303^{* * *}$ & $-0.227^{* * *}$ & $-0.288^{* * *}$ \\
& $(-3.19)$ & $(-4.043)$ & $(-3.361)$ & $(-4.566)$ & $(-3.408)$ & $(-4.055)$ \\
d_estry & $0.151^{*}$ & $0.178^{* *}$ & $0.165^{*}$ & 0.006 & $0.146^{*}$ & 0.137 \\
& $(1.721)$ & $(2.128)$ & $(1.953)$ & $(0.067)$ & $(1.754)$ & $(1.617)$ \\
d_2000 & 0.02 & 0.096 & 0.019 & 0.037 & -0.019 & 0.059 \\
& $(0.139)$ & $(0.657)$ & $(0.132)$ & $(0.259)$ & $(-0.133)$ & $(0.406)$ \\
d_2001 & -0.067 & 0.008 & -0.076 & $-0.204^{* *}$ & -0.102 & -0.096 \\
& $(-0.749)$ & $(0.088)$ & $(-0.83)$ & $(-2.03)$ & $(-1.105)$ & $(-1.047)$ \\
d_2002 & -0.086 & 0.013 & -0.074 & $-0.165^{* *}$ & $-0.146^{* *}$ & -0.038 \\
& $(-1.313)$ & $(0.186)$ & $(-1.19)$ & $(-2.518)$ & $(-2.07)$ & $(-0.608)$ \\
d_2003 & -0.025 & 0.053 & -0.04 & 0.004 & -0.024 & -0.048 \\
& $(-0.496)$ & $(0.906)$ & $(-0.784)$ & $(0.083)$ & $(-0.487)$ & $(-0.982)$
\end{tabular}

White's robust $t$-values are in parentheses

$*, * *$, and $* * *$ denote significance at the $0.10,0.05$ and 0.01 levels respectively

Table III.15. Fixed Effect OLS Results Maximum WQ

\begin{tabular}{lcccccc|} 
Variable & WTemp & pH & $\underline{\text { WVis }}$ & $\underline{\text { Salinit }}$ & $\underline{\text { DO }}$ & $\underline{\text { Grade }}$ \\
\hline WQ & $-0.033^{* * *}$ & 0.031 & -0.001 & -0.006 & $-0.054^{* * * *}$ & -0.003 \\
& $(-2.773)$ & $(0.264)$ & $(-0.542)$ & $(-1.034)$ & $(-2.928)$ & $(-0.565)$ \\
\hline d_river & $-0.282^{* * *}$ & $-0.22^{* * *}$ & $-0.234^{* * *}$ & $-0.303^{* * *}$ & $-0.188^{* * *}$ & $-0.229 * * *$ \\
& $(-3.912)$ & $(-3.249)$ & $(-3.173)$ & $(-2.685)$ & $(-2.756)$ & $(-3.299)$ \\
d_estry & $0.193^{* *}$ & $0.161^{*}$ & $0.153^{*}$ & 0.135 & $0.206^{* *}$ & $0.157 *$ \\
& $(2.258)$ & $(1.895)$ & $(1.743)$ & $(1.455)$ & $(2.384)$ & $(1.861)$ \\
d_2000 & 0.057 & 0.013 & 0.041 & 0.046 & 0.016 & 0.038 \\
& $(0.399)$ & $(0.089)$ & $(0.27)$ & $(0.322)$ & $(0.114)$ & $(0.255)$ \\
d_2001 & -0.059 & -0.066 & -0.056 & -0.015 & -0.065 & -0.051 \\
& $(-0.662)$ & $(-0.724)$ & $(-0.603)$ & $(-0.157)$ & $(-0.723)$ & $(-0.521)$ \\
d_2002 & -0.084 & -0.077 & -0.068 & -0.041 & $-0.12 *$ & -0.074 \\
& $(-1.368)$ & $(-1.255)$ & $(-1.079)$ & $(-0.623)$ & $(-1.905)$ & $(-1.211)$ \\
d_2003 & $-0.133 * *$ & -0.028 & -0.01 & -0.035 & -0.03 & -0.008 \\
& $(-2.124)$ & $(-0.578)$ & $(-0.17)$ & $(-0.727)$ & $(-0.633)$ & $(-0.144)$
\end{tabular}

White's robust $t$-values are in parentheses

$*$, **, and $* * *$ denote significance at the $0.10,0.05$ and 0.01 levels respectively 
Table III.16. Fixed Effect WQ Measure Results by Statistical Descriptor

\begin{tabular}{|c|c|c|c|c|}
\cline { 2 - 5 } \multicolumn{1}{c|}{} & \multicolumn{4}{c|}{ WQ Measure For Year of Sale } \\
\cline { 2 - 5 } \multicolumn{1}{c|}{} & Minimum & Median & Range & Maximum \\
\hline WTemp & & & & $* * *$ \\
\hline pH & $* *$ & & $* *$ & \\
\hline WVis & & $* * *$ & & \\
\hline Salinity & $* * *$ & & $* * *$ & \\
\hline DO & & $* * *$ & $* *$ & $* * *$ \\
\hline Grade & $* * *$ & $* * *$ & $* * *$ & \\
\hline
\end{tabular}

$*, * *$, and $* * *$ denote significance at the $0.10,0.05$ and 0.01 levels respectively 
Figure III.1. Study Area of Martin County, FL

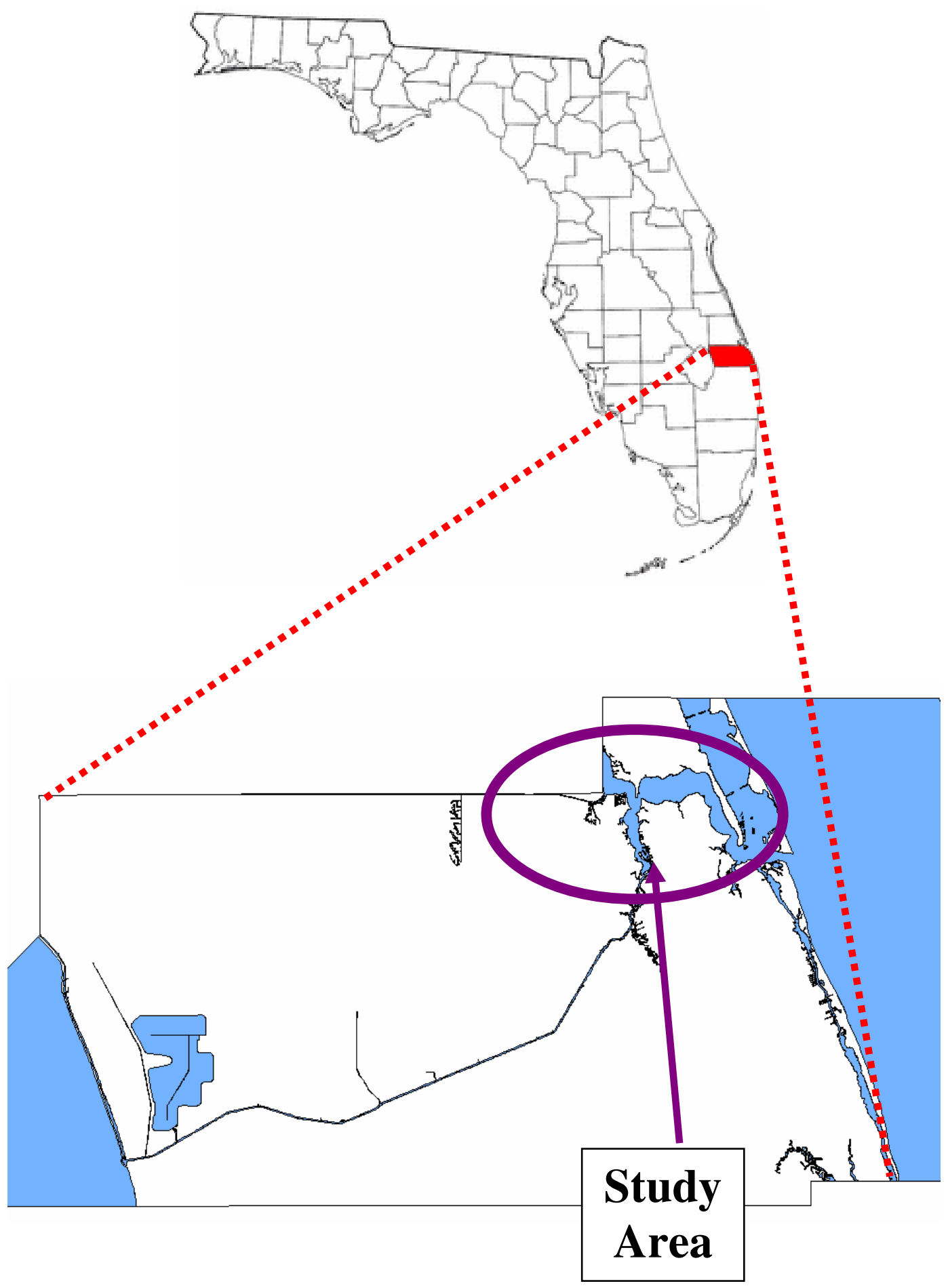


Figure III.2. Martin County Waterfront Transactions January 2000 to August 2004

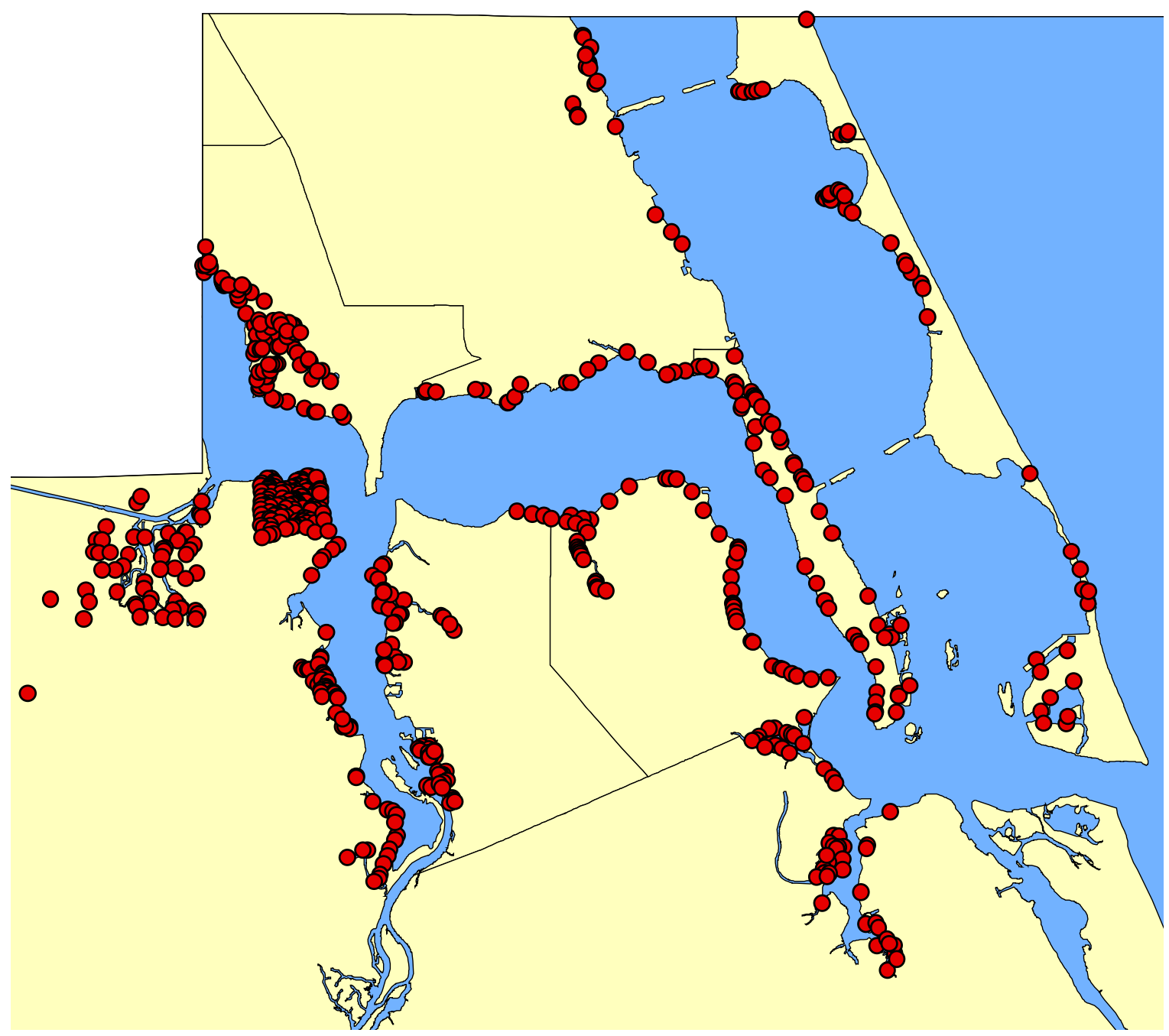

Note: Homes that sold more than one time over the study timeframe are only depicted once in the above figure 
Figure III.3. Water Quality Monitoring Locations

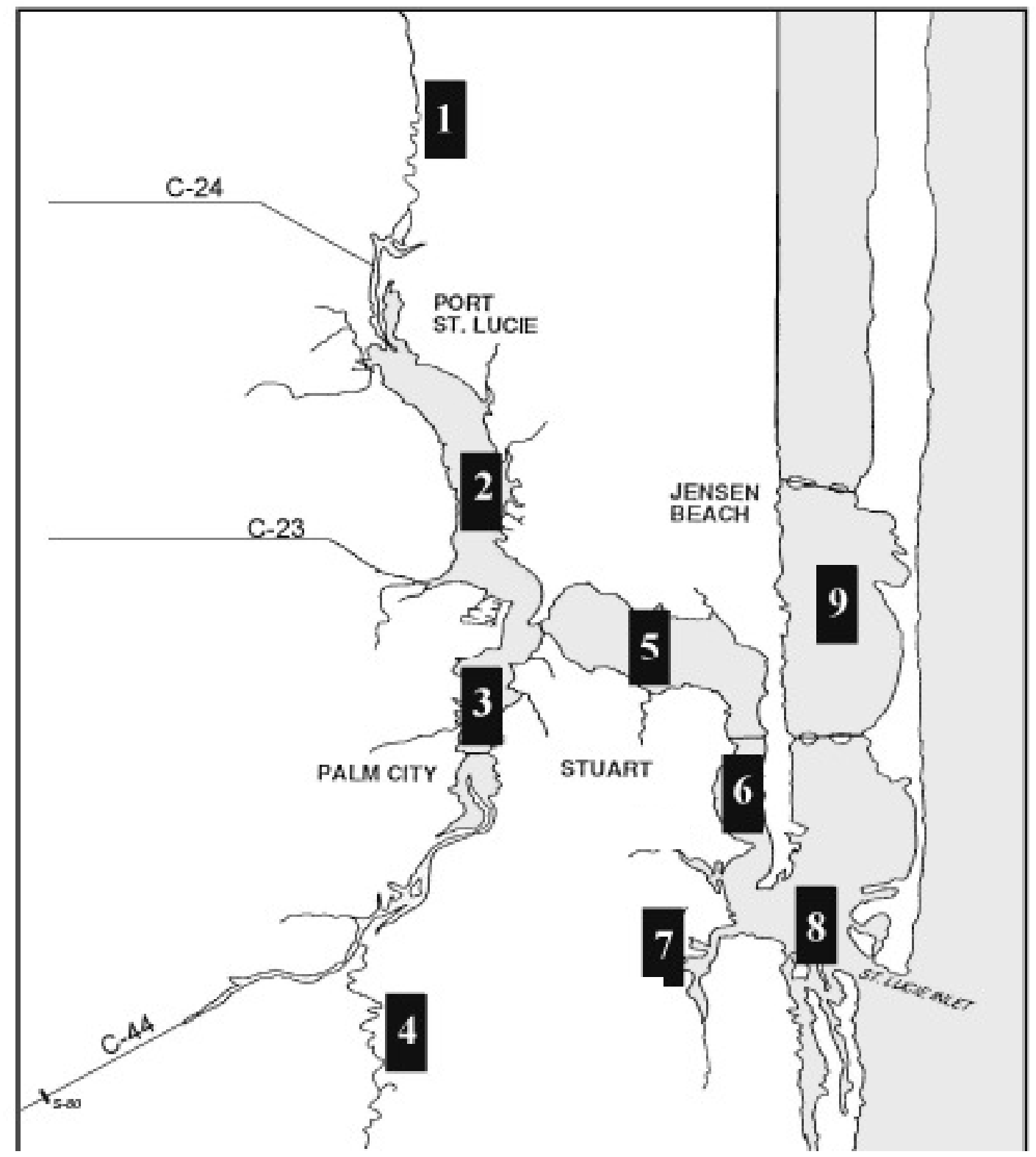

1: Winding North Fork

2: North Fork

3: South Fork

4: Winding South Fork

5: Wide Middle River
6: Narrow Middle River

7: Manatee Pocket

8: Inlet Area

9: Indian River Lagoon

(Source: FOS, 2004) 
Figure III.4. Example of Martin County Available Weekly Water Quality Data

$$
\begin{gathered}
\text { St. Lucie River Estuary Water Quality Outlook } \\
\text { Overall Grade: } 84 \% \mathrm{C}+ \\
\text { Posted: } 2 / 11 / 2004
\end{gathered}
$$

\begin{tabular}{|c|c|c|c|c|c|c|}
\hline Location & $\begin{array}{l}\text { Water } \\
\text { Temp. }\end{array}$ & $\mathrm{pH}$ & $\begin{array}{c}\text { Water } \\
\text { Visibility }\end{array}$ & Salinity & $\begin{array}{l}\text { Dissolved } \\
\text { Oxygen }\end{array}$ & $\begin{array}{c}\text { Location } \\
\text { Grade }\end{array}$ \\
\hline $\begin{array}{l}\text { 1. Winding } \\
\text { North Fork }\end{array}$ & $64^{\circ} \mathrm{F}$ & $\begin{array}{c}7.6 \\
\text { Good }\end{array}$ & $\begin{array}{c}\text { No } \\
\text { Value }\end{array}$ & $\begin{array}{c}2.8 \mathrm{ppt} \\
\text { Good }\end{array}$ & $\begin{array}{c}8.2 \mathrm{mg} / \mathrm{L} \\
\text { Good }\end{array}$ & $100 \%$ \\
\hline 2. North Fork & $55^{\circ} \mathrm{F}$ & $\begin{array}{c}8.0 \\
\text { Good }\end{array}$ & $\begin{array}{c}36.4 \% \\
\text { Fair }\end{array}$ & $\begin{array}{l}7.4 \mathrm{ppt} \\
\text { Good }\end{array}$ & $\begin{array}{c}7.9 \mathrm{mg} / \mathrm{L} \\
\text { Good }\end{array}$ & $88 \%$ \\
\hline 3. South Fork & $72^{\circ} \mathrm{F}$ & $\begin{array}{c}7.8 \\
\text { Good }\end{array}$ & $\begin{array}{c}60.4 \% \\
\text { Fair } \\
\end{array}$ & $\begin{array}{c}5.8 \mathrm{ppt} \\
\text { Good }\end{array}$ & $\begin{array}{c}7.8 \mathrm{mg} / \mathrm{L} \\
\text { Good }\end{array}$ & $88 \%$ \\
\hline $\begin{array}{l}\text { 4. Winding } \\
\text { South Fork }\end{array}$ & $72^{\circ} \mathrm{F}$ & $\begin{array}{c}7.6 \\
\text { Good }\end{array}$ & $\begin{array}{l}44.4 \% \\
\text { Fair }\end{array}$ & $\begin{array}{c}0 \mathrm{ppt} \\
\text { Below Range }\end{array}$ & $\begin{array}{c}6.7 \mathrm{mg} / \mathrm{L} \\
\text { Good }\end{array}$ & $63 \%$ \\
\hline $\begin{array}{l}5 . \quad \text { Wide } \\
\text { Middle River }\end{array}$ & $72^{\circ} \mathrm{F}$ & $\begin{array}{c}8.1 \\
\text { Good } \\
\end{array}$ & $\begin{array}{l}69.2 \% \\
\text { Good } \\
\end{array}$ & $\begin{array}{c}9 \mathrm{ppt} \\
\text { Below Range }\end{array}$ & $\begin{array}{c}8.2 \mathrm{mg} / \mathrm{L} \\
\text { Good }\end{array}$ & $75 \%$ \\
\hline $\begin{array}{l}\text { 6. Narrow } \\
\text { Middle River }\end{array}$ & \multicolumn{6}{|c|}{ Not Reporting } \\
\hline $\begin{array}{c}\text { 7. Manatee } \\
\text { Pocket }\end{array}$ & $68^{\circ} \mathrm{F}$ & $\begin{array}{c}8.1 \\
\text { Good }\end{array}$ & $\begin{array}{l}73.2 \% \\
\text { Good }\end{array}$ & $\begin{array}{c}17.8 \mathrm{ppt} \\
\text { Below Range }\end{array}$ & $\begin{array}{c}6.5 \mathrm{mg} / \mathrm{L} \\
\text { Good }\end{array}$ & $75 \%$ \\
\hline
\end{tabular}

8. Inlet Area

Not Reporting

\begin{tabular}{lcccccc}
\hline 9. IRL & $73.4^{\circ} \mathrm{F}$ & $\begin{array}{c}8.2 \\
\text { Good }\end{array}$ & $\begin{array}{c}\text { No } \\
\text { Value }\end{array}$ & $\begin{array}{c}32 \mathrm{ppt} \\
\text { Good }\end{array}$ & $\begin{array}{c}7.1 \mathrm{mg} / \mathrm{L} \\
\text { Good }\end{array}$ & $100 \%$ \\
\hline
\end{tabular}

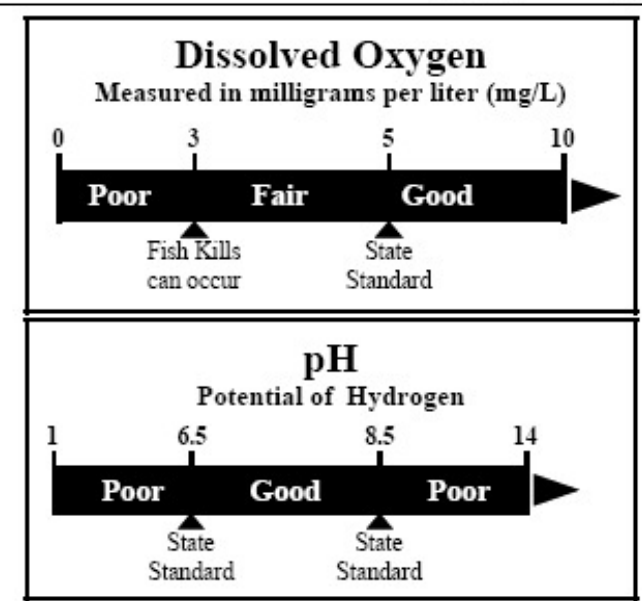

Comment: The data above may indicate areas of concern in the St. Lucie Estuary. Citizens should call the Florida Department of Environmental Protection (DEP) at 871-7662 or the South Florida Water Management District (SFWMD) 223-2600 to ask about the quality of a specific area and report observations of pollution.

\begin{tabular}{|c|c|}
\hline$\& 5$. & $0.2-14.9$ \\
\hline $\begin{array}{l}\text { Inner Estuary } \\
\text { (N. \& S. Fork) }\end{array}$ & $5-24.9$ \\
\hline $\begin{array}{l}\text { Middle Estuary } \\
\text { (Roosevelt Bridge to Hells Gate) }\end{array}$ & $15-30$ \\
\hline $\begin{array}{l}\text { Outer Estuary } \\
\text { (Hells Gate to Inlet) }\end{array}$ & -36 \\
\hline $\begin{array}{l}\text { Indian River Lagoon } \\
\text { (Stuart Causeway to Je }\end{array}$ & $\begin{array}{l}25-36 \\
\text { Causewa }\end{array}$ \\
\hline
\end{tabular}

Salinity Ranges

Measured in parts per thousand (ppt)

Water Visibility

Depth into the water that you can see. Represents the amount of suspended material

in the water. The higher the number the clearer the water.

$\%$ Visibility $=$ Secchi Depth $/$ Water Depth

Graded relative to depth at site

Visibility less than $33 \%$ depth to bottom $=$ Poor Visibility between $33 \%$ and $66 \%$ depth to bottom = Fair Visibility more than $66 \%$ depth to bottom $=$ Good No Value $=$ Water too shallow for reading

(Source: Modified from FOS, 2004) 
Figure III.5. DO Levels by WQ Monitoring Location from Jan 2000 to Dec 2004

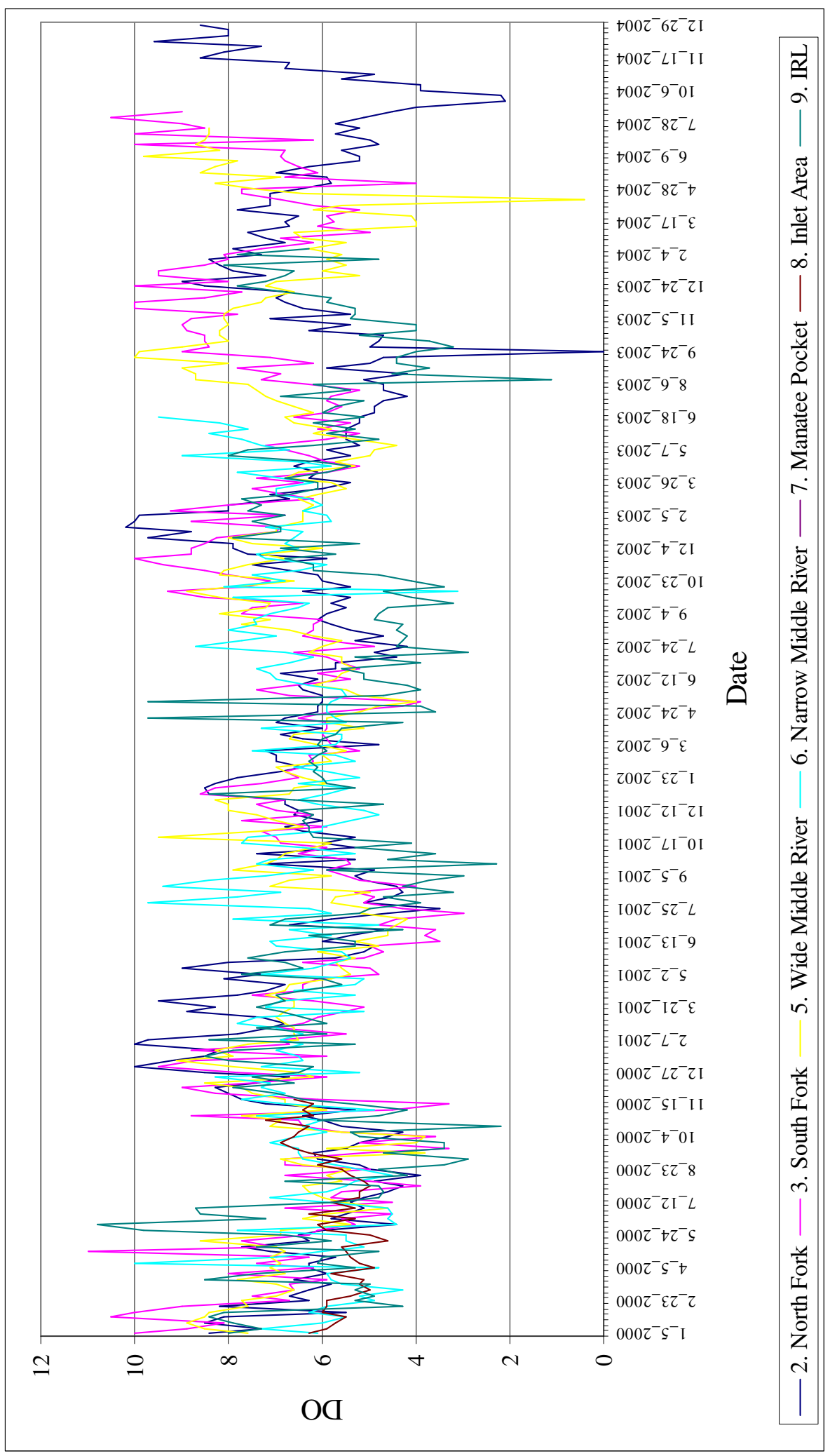


Figure III.6. Waterfront Home Sales by WQ Monitoring Location

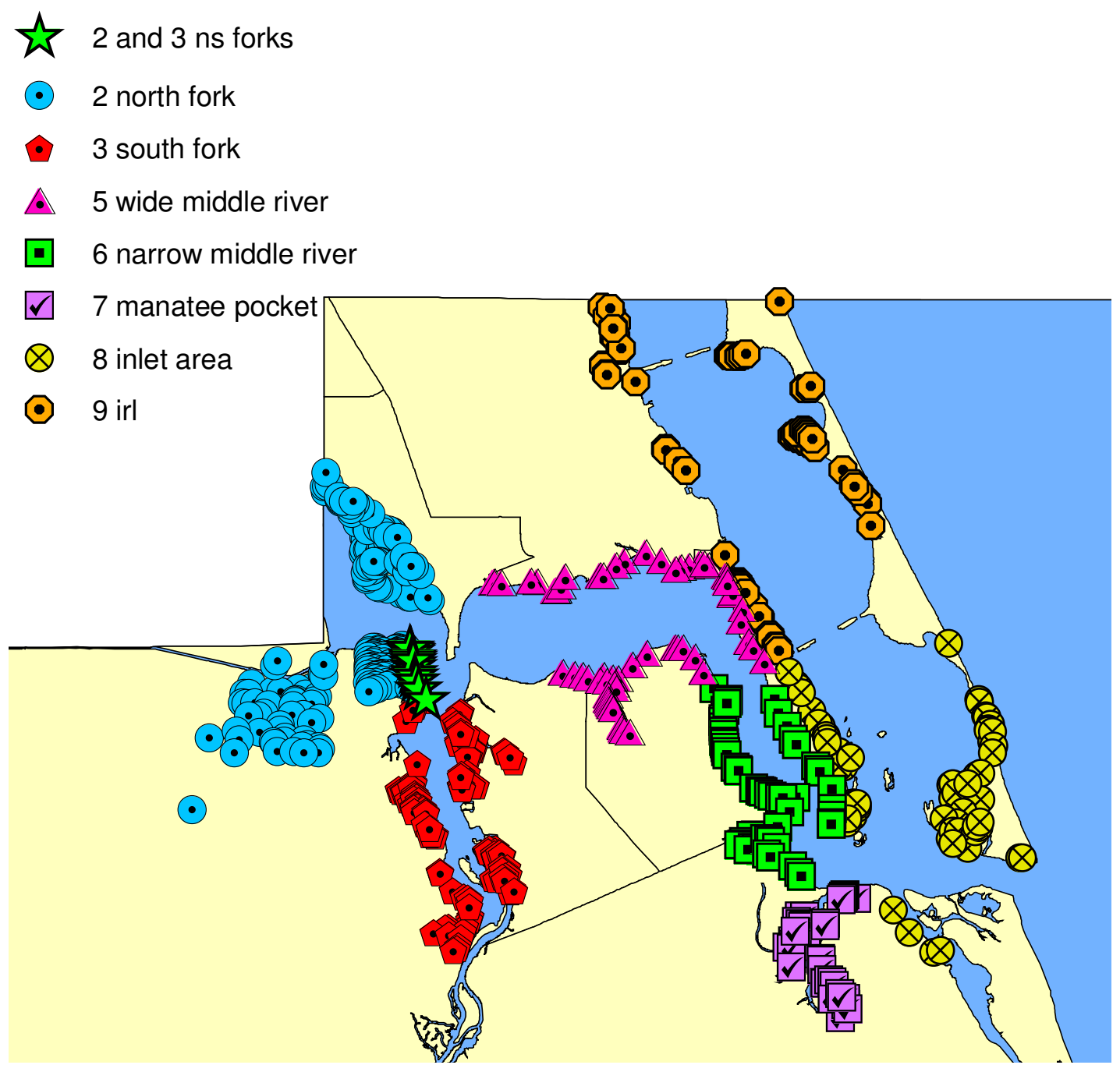


Figure III.7. a) Median Water Visibility by Year \& Location; b) Min, Avg, \& Max Water Visibility by Location for Jan 2000 to Dec 2004
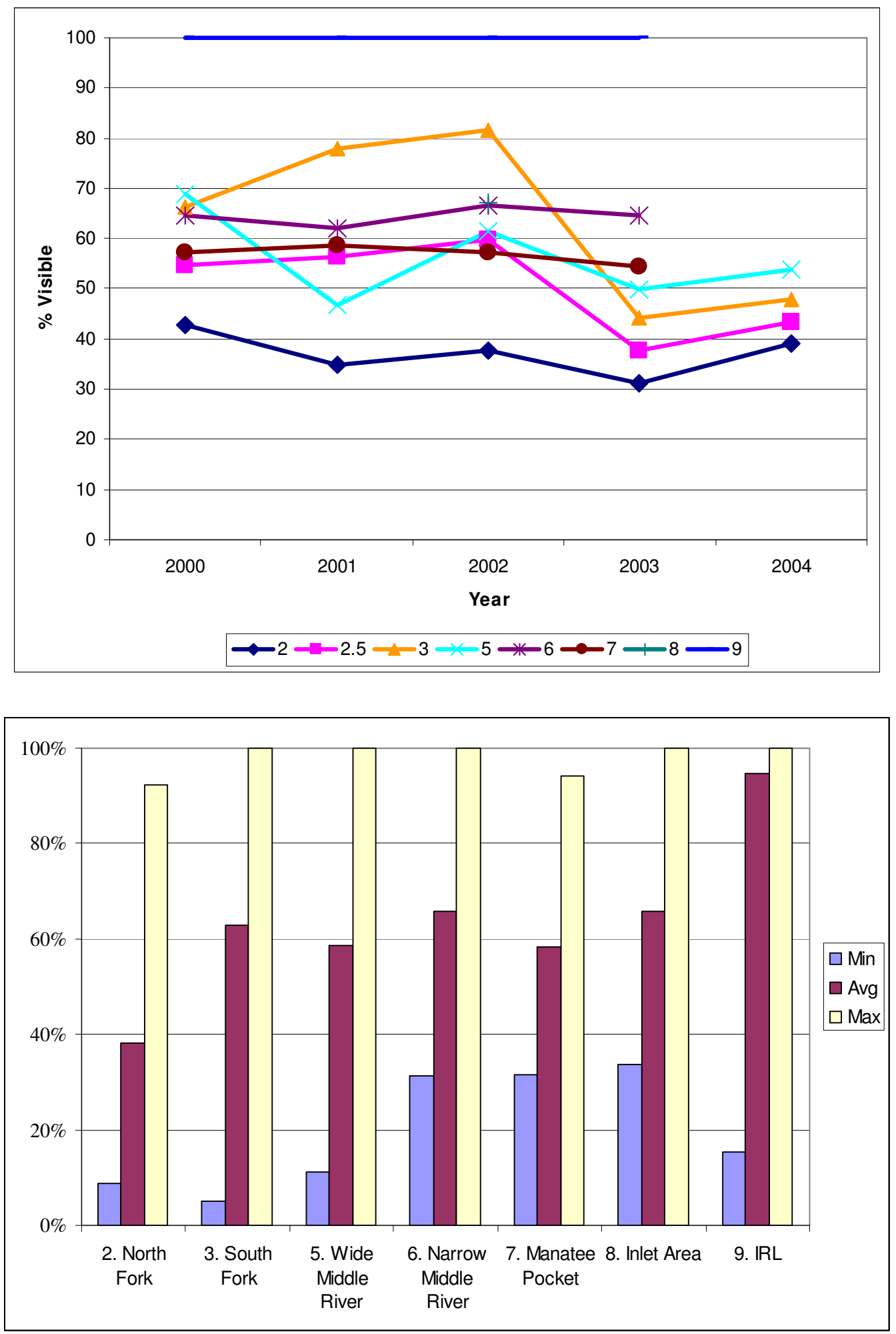


\section{IS IT TIME TO GO YET? DYNAMICALLY MODELING HURRICANE EVACUATION DECISIONS}

\section{IV.I. Introduction}

The 2005 hurricane season was the most active hurricane season on record and left in its wake many poignant reminders concerning the critical role of evacuation, including the floodwater trapped citizens of New Orleans due to Hurricane Katrina, as well as the evacuation gridlock outside Houston caused by the impending Hurricane Rita. Projections of future hurricane seasons currently indicate continued high levels of hurricane activity, further emphasizing the need to better understand evacuation behavior in order to attempt to avoid similar evacuation breakdowns. Much of the evacuation research to date has focused on either the characteristics of those who evacuate, such as location/type of home, income level, etc., or the general difficulties associated with evacuating (Dash and Gladwin, 2005). In their overview of social science research needs related to hurricane forecasts and warnings, Gladwin et al. (2005, pg. 9) highlight the need for research that leads to "... modeling of evacuation behavioral response in more precise and comprehensive ways". Enhanced hurricane evacuation modeling in regard to the incorporation of decision makers' risk perceptions, beliefs, constraints, and abilities to decipher warning signals, as well as the dynamic nature of decision making in a nonlinear communication environment are all repeated and accentuated themes resulting from the white papers associated with the Gladwin et al. (2005) report.

The purpose of this paper is to respond to one of the hurricane evacuation issues by developing a dynamic model of hurricane evacuation behavior. Specifically, a household's evacuation decision is framed as an optimal stopping problem where every 
potential evacuation time period prior to the actual hurricane landfall, the household's optimal choice is either to evacuate, or to wait one more time period for a revised hurricane forecast. Given this theoretical framework, relevant policy questions that affect the timing of the household evacuation decision are analyzed.

In order to provide the intuition behind evacuation decision making in a dynamic framework, we present initial results from a simple, two-period model of evacuation. By utilizing the two-period model to contrast a dynamic vs. static "now or never" view of the optimal $1^{\text {st }}$ period evacuation decision, we show that the dynamic perspective results in $1^{\text {st }}$ period waiting being optimal for certain levels of evacuation costs, even thought $1^{\text {st }}$ period evacuation is optimal from a static perspective. We illustrate other distinct dynamic vs. static optimal waiting/evacuating results for a number of possible hurricanerelated policies such as a mitigation focus solely on potential damage from the more frequent, but less destructive minor hurricanes. Further benefits of the use of a dynamic framework to analyze evacuation behavior are highlighted with the simple, two-period model including an analysis of households with heterogeneous evacuation costs, and the ability of the two-period results to provide a deeper understanding of existing evacuation timing empirical outcomes.

However, as we ultimately want to better explain actual evacuation behavior, we extend our hypothetical two-period model to a realistic multi-period setting that is calibrated using existing forecast and evacuation cost data for a specific region, coastal areas on the Gulf of Mexico. We show that the model does a good job of explaining actual evacuation behavior in specific hurricanes, as well as expected evacuation timing outcomes by various household types. From this calibrated dynamic framework, a 
number of policy questions that plausibly affect the timing of household evacuations are analyzed. For example, does building more shelters induce earlier evacuation? Additionally, the value of an improved hurricane forecast is an outcome of the policy analysis.

Whitehead (2003) estimates the probability of evacuation for varying levels of hurricane intensity, but does so from a static perspective as the timing of the probability of an evacuation for any particular storm intensity level is not addressed. However, the evacuation decision when faced by a hurricane threat has the three qualities of irreversibility, uncertainty, and the ability to wait for more information that characterize a decision process that is better understood from a dynamic modeling approach (Dixit and Pindyck, 1994). Standard empirical results from the evacuation literature such as the traditional S-shaped evacuation response curves (USACE, 2006a) indicate certain households wait while others evacuate, and therefore further underscore the need for a dynamic perspective of evacuation behavior. Likewise, only from a dynamic perspective can it be shown (as we do) that households may in fact be acting rationally by choosing to ignore evacuation warnings and waiting for more information, even though it could be the case that the benefits of evacuating (i.e., the expected avoided damage costs) outweigh the evacuation costs for a particular static snapshot of time - a situation that seemingly perplexes public evacuation authorities.

Moreover, modeling the evacuation decision process dynamically over many time periods with households having the ability to wait for more information, is analogous to a real-life evacuation decision situation where the National Hurricane Center (NHC) issues official forecast advisories every six hours once a tropical depression, tropical storm, or 
hurricane has developed. While Fu and Wilmot (2004) utilize a sequential choice model to estimate the probability of a household evacuating or waiting in each period of their dynamic multi-period framework, and further use their dynamic model results to provide clarification to the standard evacuation timing empirical outcomes, their research differs from ours in a number of significant ways. Importantly, we provide a theoretical model of dynamic evacuation behavior which is necessary for conducting policy analysis. Furthermore, our dynamic model is calibrated with forecast data from a number of storms across a number of locations which coincides directly with the six hour NHC forecast advisory timeline, and we explicitly address the costs of evacuation in a household's evacuation decision. Our research then serves as a contrast to the existing models of hurricane evacuation behavior by utilizing a theoretically-driven dynamic modeling approach that provides a more realistic interpretation to the multi-period evacuation decision process through the use of forecast and evacuation cost data, thereby helping to further bridge the knowledge gap between hurricane forecasts and evacuation timing behaviors.

The remainder of the paper is organized as follows: Section II provides an overview of the uncertainty inherent in hurricane forecasts and its effect on the timing of evacuations; Section III presents and solves a two-period evacuation decision model, building the intuition for the results to the multi-period model; Section IV describes the optimal stopping problem for the multi-period evacuation decision setting; Section V discusses the calibration of this model's inputs and parameters; Section VI provides the model's recursive solution and results; Section VII discusses policy implications from the multi-period results; and Section VIII gives the concluding comments. 


\section{IV.II. The Uncertain Nature of Hurricane Forecasts and Evacuation Timing}

Once a tropical depression, tropical storm, or hurricane has developed, the NHC issues an official forecast advisory every six hours at 5:00 a.m., 11:00 a.m., 5:00 p.m., and 11:00 p.m. ${ }^{30}$. While the accuracy of the information contained in the NHC forecast advisories has improved, and continues to improve over time, the existing science around forecasting hurricane tracks and intensity levels is such that forecasts contain much uncertainty $^{31}$. Assuming that for any particular six-hour time period prior to a hurricane's landfall with an associated NHC forecast advisory, households are using the information contained in the current forecast advisory to decide whether to evacuate immediately, or to wait for the next advisory containing an updated hurricane forecast, it is not surprising to see empirical evidence suggesting heterogeneous evacuation behaviors among households where some evacuate while others wait ${ }^{32}$. This heterogeneous evacuation behavior is a function of the inherent uncertainty contained in the $\mathrm{NHC}$ forecast information that accordingly places a great deal of uncertainty into a household's decision to evacuate during any particular time-period.

\footnotetext{
${ }^{30}$ Occasionally, an additional intermediate advisory will be issued as a storm is getting closer to landfall. As this is a rare occurrence, and furthermore as not all of the forecast information contained in the intermediate advisory changes from the normal six-hour advisory, we do not consider it further.

${ }^{31}$ We consider only the intrinsic technical uncertainty of the forecast in our analysis. However, in addition to the technical uncertainty of each forecast, it needs to be pointed out that there is also a level of subjective uncertainty that is a function of the hurricane forecasting process. Each official forecast released by the $\mathrm{NHC}$ is ultimately a compilation of a number of different forecasting models available to the NHC forecaster responsible for that particular storm. Of course, all of these models are never in full agreement on exactly what the storm will do. The forecaster consequently places a subjective level of confidence on what he believes to be the most correct information coming from the various available models, and also based on his past forecasting experience.

${ }^{32}$ Regardless of whether households are using the NHC forecast advisories to base their evacuation decision upon, whatever source of information they are using will still contain some level of uncertainty. Furthermore, it is the case that "... almost all hurricane forecast information the public receives is a repackaged form of NHC data” (Regnier, pg. 3, 2006).
} 


\section{NHC Forecast Advisories}

The most critical aspects of information contained in the NHC forecast advisory (which are also distinct from information contained in any of the other hurricane products issued by the NHC) include a 12, 24, 36, 48, 72, 96, and 120 hour forecast $^{33}$ of an approaching hurricane's center position (track forecast), maximum one-minute (i.e., sustained) wind speeds (intensity forecast), non-sustained wind speed gusts, and asymmetrical maximum wind radii from the storm's center of minimum tropical storm strength $(34 \mathrm{KT})$, strong tropical storm strength $(50 \mathrm{KT})$, and hurricane force (64 KT) winds $^{34}$ (NHC, 2006). An example of this information from Hurricane Dennis's forecast advisory \#19 issued at 5:00 a.m. on July 9, 2004 is presented in Figure 1, with the "forecast valid" at 09/1800Z, 10/0600Z, 10/1800Z, 11/0600Z, 12/0600Z, 13/0600Z, and $14 / 0600 Z$ denoting the $12,24,36,48,72,96$, and 120 hour forecast respectively ${ }^{35}$. In addition to this forecast information, other information contained in the NHC forecast advisories (which is mostly non-distinct from information contained in any of the other hurricane products issued by the NHC), includes any watches and warnings issued, as well as the current center location, center accuracy, movement, pressure, eye diameter, maximum winds, and wind radii.

The track and intensity forecasts are subsequently evaluated against "best-track" storm data during a post-storm analysis, where track forecast error and forecast intensity

\footnotetext{
3396 and 120 hour forecasts began in 2001, but were not made part of the official forecast advisory available to the public until 2003 (http://www.nhc.noaa.gov/verification/verify2.shtml?)

${ }^{34}$ Radii distance is given in nautical miles, and the maximum wind radii are not given for the 96 and 120 hour forecasts.

${ }^{35}$ One will note that the $09 / 1800 Z 12$ hour forecast equates to 2:00 p.m., whereas the current advisory is for 5:00 a.m., which is a 9 hour difference, not 12 hours. This is because the forecast is actually based on synoptic time data which is collected three hours prior to the advisory time (Norcross, 2006).
} 
error are determined. Track forecast error is defined as "as the great-circle distance between a cyclone's forecast position and the best track position at the forecast verification time", and forecast intensity error is defined as "the absolute value of the difference between the forecast and best track intensity at the forecast verifying time" (NHC, 2006). Figure 2 provides the annual average official track forecast errors for Atlantic basin tropical storms and hurricanes from 1970 through $2005^{36}$, and Figure 3 provides the annual average intensity errors for Atlantic basin tropical cyclones from 1990 through $2005^{37}$.

Figure IV.1. Hurricane Dennis Forecast Advisory \#19

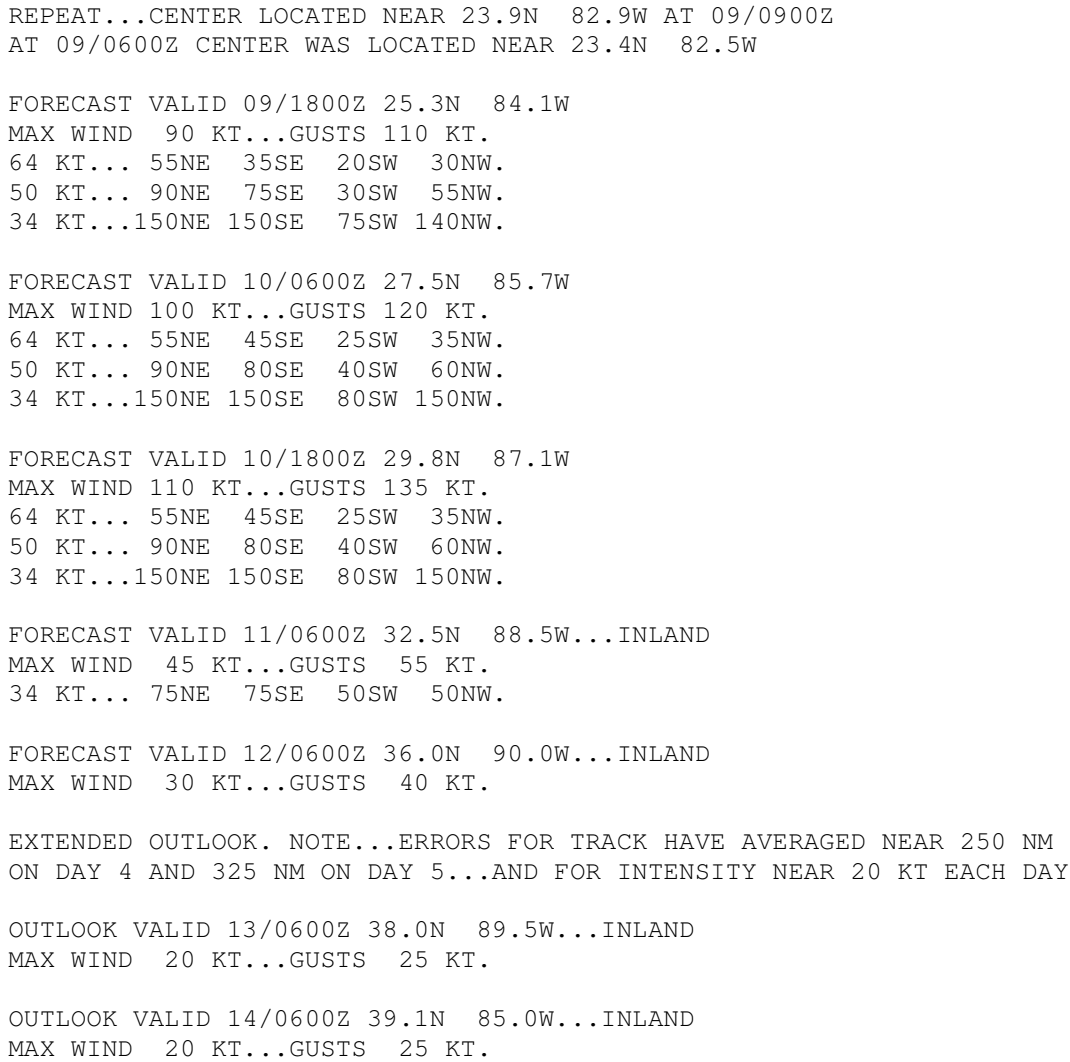

(Source: NHC, 2006)

\footnotetext{
${ }^{36}$ Track forecast errors for the Eastern North Pacific Basin are similar (NHC, 2006)

${ }^{37}$ Intensity errors for the Eastern North Pacific Basin are similar (NHC, 2006)
} 
While it can be seen in Figure 2 that the track forecast errors have improved over time, there is still a high amount of variability in the accuracy of the forecast track as the number of hours prior to landfall increases. For example, from Figure 2 in 2004 the average track forecast error decreases nearly $80 \%$ from 323 miles 120 hours out from landfall, to 62 miles 24 hours out from landfall. A further illustration of the magnitude of the track forecast errors over time is shown through an example of the NHC's wellknown forecast cone in Figure 4. Using the 12, 24, 36, 48, 72, 96, and 120 hour forecast as the center point of the storm, the forecast cone is simply constructed by drawing a circle with radius equal to the average track forecast error over the previous five years around each forecast point (Norcross, 2006). Figure 4 clearly shows how the diameter of the cone expands with forecast time, where 120 hours out from potential landfall it is wide enough to come into contact with four out of the possible five states along the Gulf Coast!

Figure IV.2. NHC Official Annual Average Track Errors, Atlantic Basin Storms

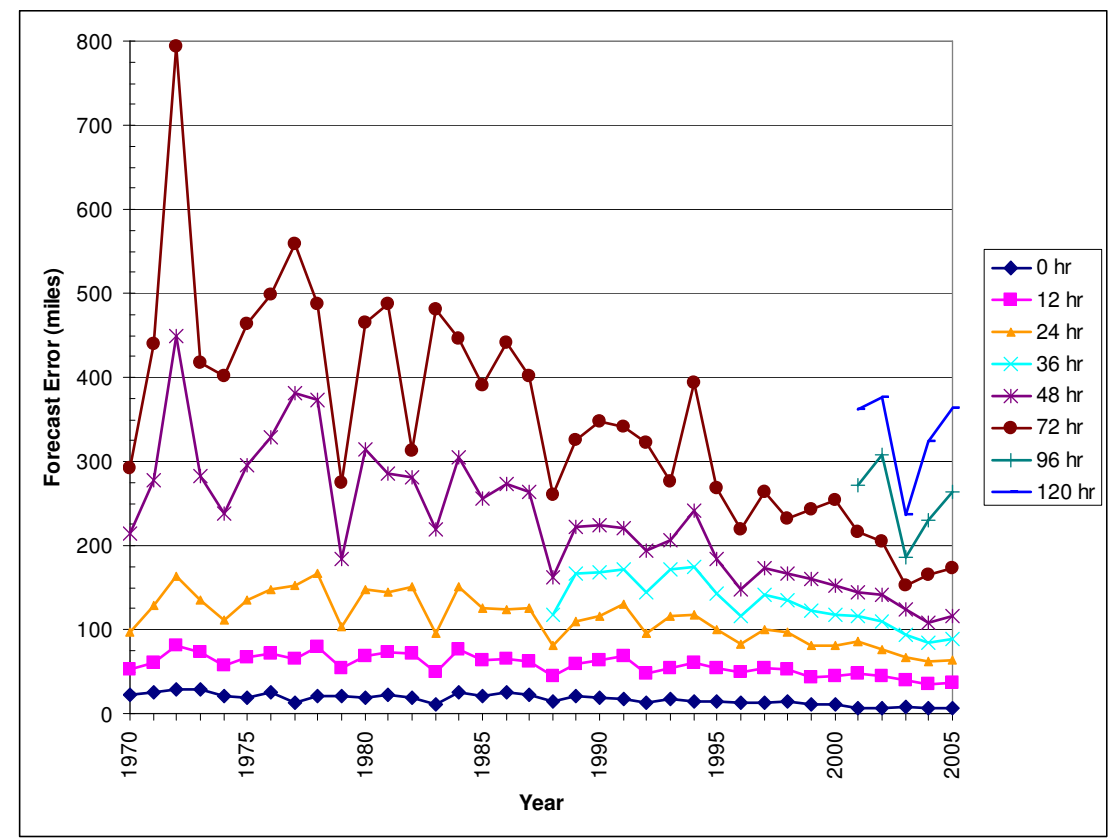

(Source: Adapted from NHC, 2006) 
Similarly for intensity errors, the same accuracy variability story arises. Storm intensity is measured through the five category levels of the Saffir-Simpson hurricane scale (SSHS) as presented in Table 1. In 2004, 120 hours from landfall the average intensity error is $26 \mathrm{mph}$, which is sufficient magnitude to plausibly cross three categories on the SSHS. 24 hours out from landfall, the intensity error has decreased by nearly $55 \%$ to approximately $11.5 \mathrm{mph}$, which, despite the error decrease, is still enough to straddle two SSHS category levels.

Table IV.1. Saffir-Simpson Hurricane Scale

\begin{tabular}{|c|c|c|}
\hline $\begin{array}{c}\text { Hurricane } \\
\text { Category Level }\end{array}$ & Wind Speed $(\mathrm{mph})$ & Damage \\
\hline 1 & $74-95$ & Minimal \\
\hline 2 & $96-110$ & Moderate \\
\hline 3 & $111-130$ & Extensive \\
\hline 4 & $131-155$ & Extreme \\
\hline 5 & $>155$ & Catastrophic \\
\hline
\end{tabular}

(Source: NHC, 2006)

Figure IV.3. NHC Official Annual Avg Intensity Errors Atlantic Basin Tropical Cyclones

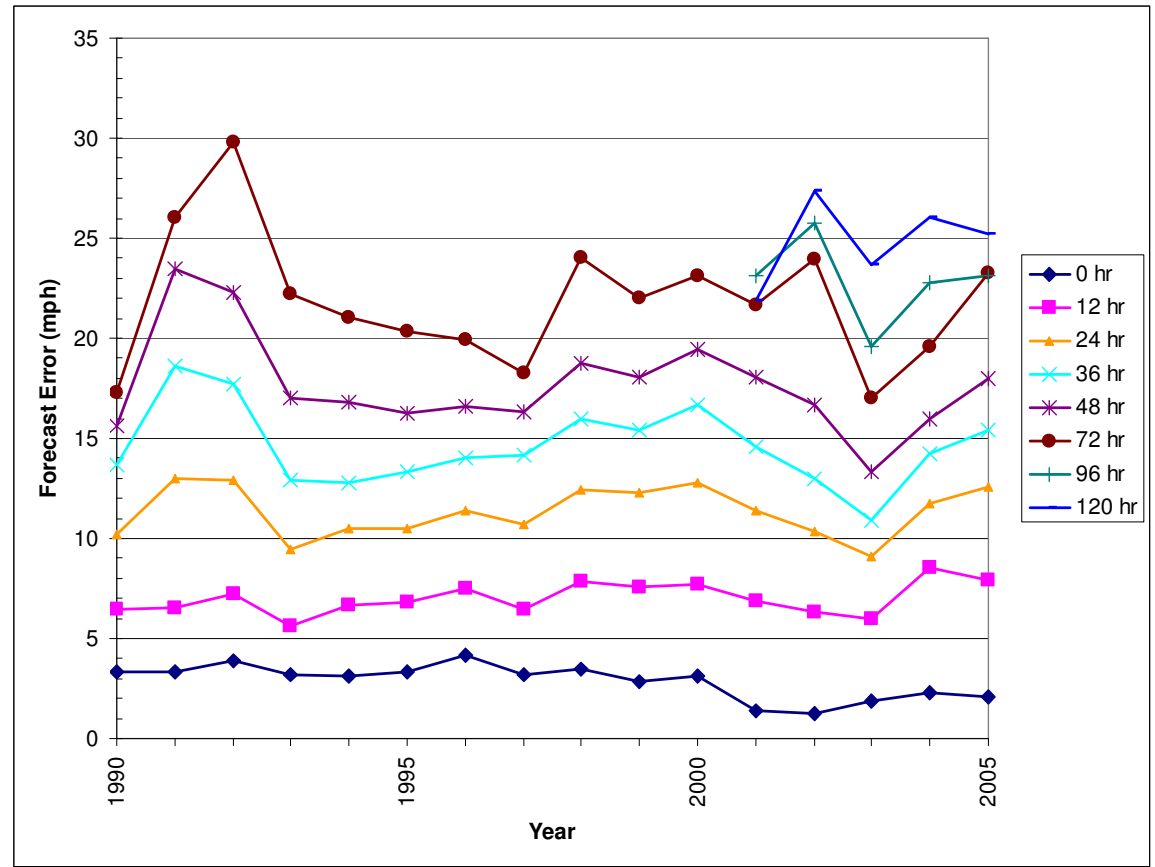

(Source: Adapted from NHC, 2006) 
Figure IV.4. NHC Average Error Cone Example

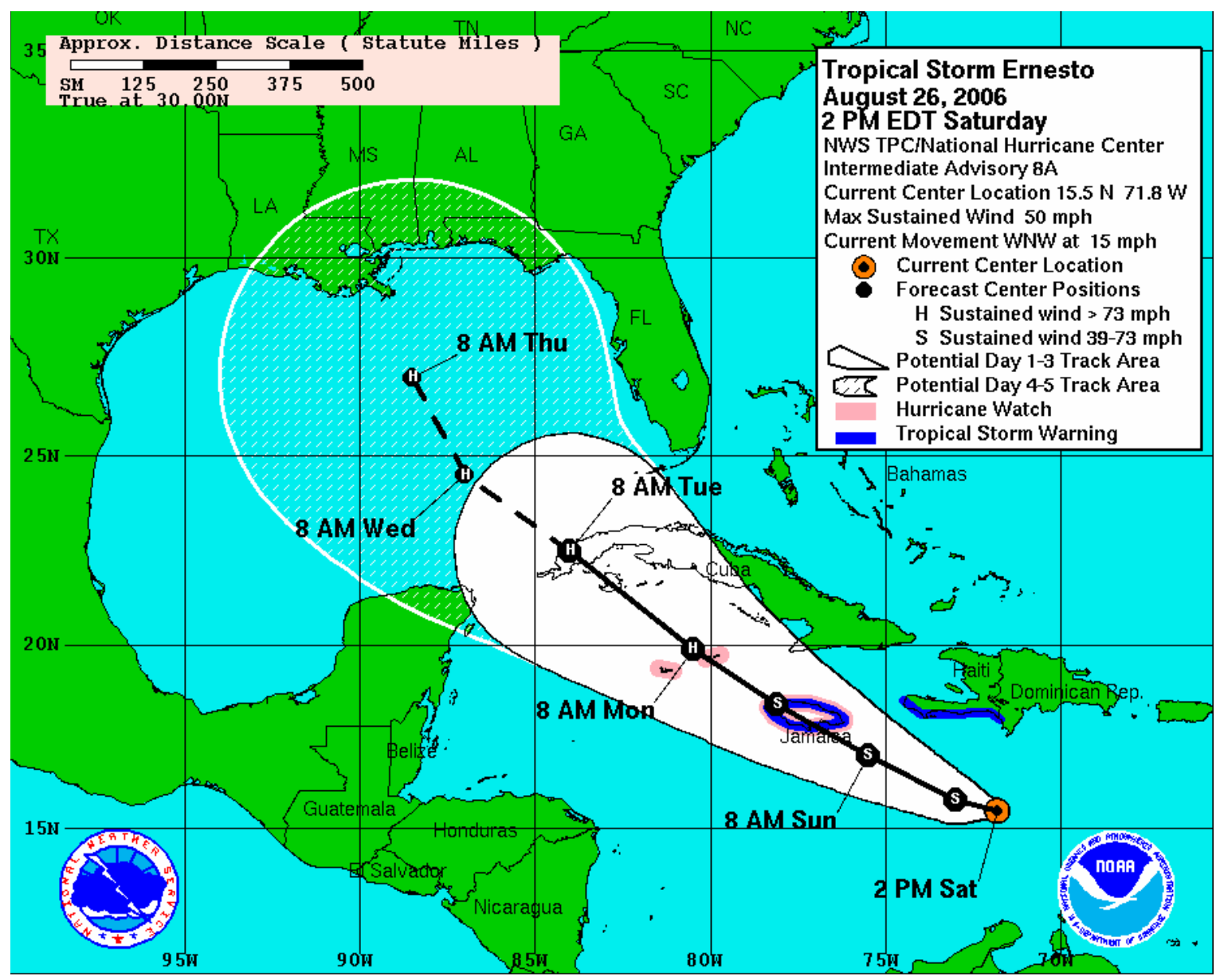

(Source: NHC, 2006)

Given that recommended safe evacuation times for major coastal communities are at least 30 hours in advance of a hurricane's expected landfall (Lindell et al., 2005a), these figures highlight the significant amount of uncertainty inherent in the hurricane forecast that households must use to make evacuation decisions during this recommended safe evacuation timeframe. For example, the 36 hour forecast in 2005 had a track forecast error of 90 miles, and an intensity error of $15 \mathrm{mph}$. Imagine a location that is within the average error forecast cone for the 36 hour forecast, but it is located 80 miles east of the forecasted center of the storm, and is therefore on the far eastern side of the 
cone (assume the hurricane and hence the cone are moving south to north). Let the 36 hour forecast call for an intensity of $105 \mathrm{mph}$ at landfall, which is a category 2 (CAT 2) hurricane. Assuming the storm actually stays within the cone 36 hours later ${ }^{38}$, it could potentially make landfall 170 miles west of our imagined location (i.e., 90 miles west of the forecasted storm center), placing our location out of harm's way ${ }^{39}$. Furthermore, even if the storm does head directly toward our location it could make landfall anywhere from $90 \mathrm{mph}$ to $120 \mathrm{mph}$, or from a CAT 1 up to a CAT 3 hurricane. As CAT 1 hurricanes are classified as causing minimal damage, and CAT 3 hurricanes as causing extensive damage, the difference in potential damage is significant.

Undoubtedly, a household's evacuation decision 36 hours from landfall knowing that a CAT 3 storm will be tracking directly over it, or a CAT 1 storm will be tracking 170 miles west of it, would be different. And the fact that the storm is forecasted to potentially fall anywhere in between these two extremes leads to inevitable uncertainty in regard to a household's decision to evacuate during this 36 hour time period.

\section{NHC Products Addressing Forecast Uncertainty}

Strike probabilities are an additional product issued by the NHC every six hours in conjunction with the forecast advisories which provide households with probabilistic information concerning the storm tracking over their specific location that the forecast advisory and the average error cone do not provide. A strike probability on a location is defined as "the percent chance that the center of the storm will cross within 65 nautical

\footnotetext{
${ }^{38}$ In fact, storms only stay within the cone $70 \%$ of the time (Norcross, 2006)

${ }^{39}$ Regnier (pg.1, 2006) states that "hurricanes affect about 150 miles of coastline per landfall"
} 
miles (75 miles) of a location" (NHC, 2006). The strike probabilities by their very nature are meant to convey the uncertainty intrinsic to the hurricane forecasts, and for households to use this information to assist in making their decision to evacuate.

A graphical example from HURREVAC software of the provided strike probabilities for the 7/9/2005 5:00 a.m. Hurricane Dennis advisory \#19 is given in Figure 5. From Figure 5, it is clear that the probability of a strike on a location is not limited to only those locations falling within the 3-day average error forecast cone, or that the strike probabilities add up to $100 \%$ across all locations. Another important point to note about the strike probabilities is that they are constrained by the NHC. For the 12, 24, 36, 48, and 72 hour forecasts, the maximum strike probability issued by the NHC is $60-80 \%, 35$ $50 \%, 20-25 \%, 13-18 \%$, and 10\% respectively (HURREVAC). Strike probabilities are not provided beyond 72 hours out from a storm.

Starting in 2006 the strike probabilities have been replaced by the NHC's new wind speed probabilities product. The wind speed probability product not only provides the probability of the storm tracking over a location, but also combines this information with wind intensity level information. In this way, the new product gives "the probability of winds of a certain strength affecting a given location" (Norcross, pg.114, 2006). Additionally, the new product is constructed based on simulated data rather than historical data, and provides information on tropical storm force winds instead of solely hurricane strength winds. Accompanying the new wind speed probabilities product is also the new wind speed probability table. This table conveys the uncertainty inherent in the intensity forecasts by providing the probability of winds being dissipated, becoming 
tropical strength, and achieving various levels of hurricane strength for the 12, 24, 36, 48, and 72 hour forecasts.

Even though the NHC issues products meant to address the uncertainty of the hurricane forecasts, this does not mean that either household uncertainty or heterogeneous evacuation behaviors disappear. Each household will ultimately interpret this information in their own subjective way ${ }^{40}$, and this information is constantly changing with every new forecast advisory. What should be clear from the above discussion is that hurricane forecasts are intrinsically uncertain, and that there are products issued by the NHC aimed at alleviating the extent of this uncertainty in order to facilitate a household's decision to evacuate or wait.

Figure IV.5. HURREVAC Strike Probabilities for Hurricane Dennis Advisory \#19

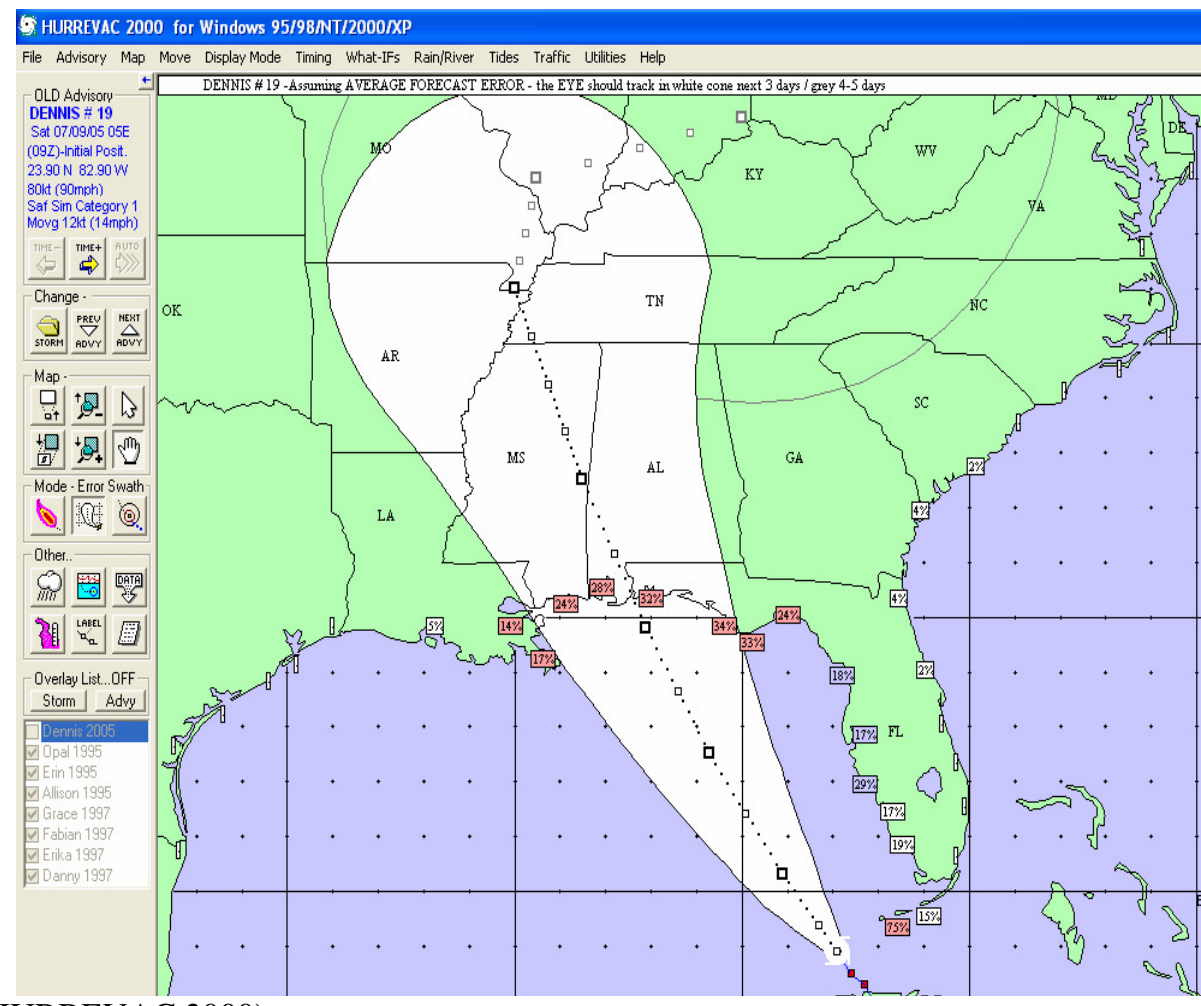

(Source: HURREVAC 2000)

\footnotetext{
${ }^{40} \mathrm{We}$ do not attempt to address the subjective beliefs of households in regard to forecasts in this study.
} 


\section{Heterogeneous Evacuation Behavior}

Again, the intrinsic and significant uncertainty of hurricane forecasts accordingly places a great deal of uncertainty into a household's decision to evacuate during any particular forecast time-period, and it is therefore not surprising to see empirical evidence suggesting heterogeneous evacuation behaviors amongst households where some evacuate while others wait. For example, Baker (2005b) found that for Hurricane Ivan more than 68 hours elapsed from the time the first person evacuated to the time the last person evacuated in the Gulf region, which equates to nearly eleven NHC forecast advisories spanning the course of three days. As he states in regard to the timing of evacuations, “... it must be recognized that evacuation is a several day process ..." (pg. 64, 2005b).

Post-storm assessments for a number of hurricanes are completed by FEMA and the USACE (USACE 2006b), where a section on evacuation timing is typically included. The cumulative evacuation timing curves that are produced as a part of these post-storm assessments always indicate heterogeneous evacuation behavior of varying degrees by those surveyed households that had decided to evacuate. On October 4, 1995 at approximately 5:00 p.m., Hurricane Opal made landfall as a strong CAT 3 hurricane over Pensacola, FL, and Figure 6 presents the cumulative evacuation response curve from the Hurricane Opal post-storm assessment. In this case, while the first households to evacuate left nearly 42 hours before landfall on Oct 3, clearly the vast majority of households left between 11:00 p.m. on Oct 3 and 11:00 a.m. on Oct. 4. Despite this relatively late evacuation response, the curve still takes on the traditionally assumed Sshape. 
Other empirical evidence has found sharp increases in evacuation response rates in relation to an official evacuation order being issued (USACE 2006a), with response rates designated as fast, medium, and slow. Figure 7 provides an example of the traditional S-shaped behavioral evacuation response curves for cumulative households depicting fast, medium, and slow evacuation response rates in relation to an official evacuation order. So not only do some households wait while others evacuate, but their rate of waiting and evacuating vary as well.

Figure IV.6. Hurricane Opal Cumulative Evacuation Response Curve

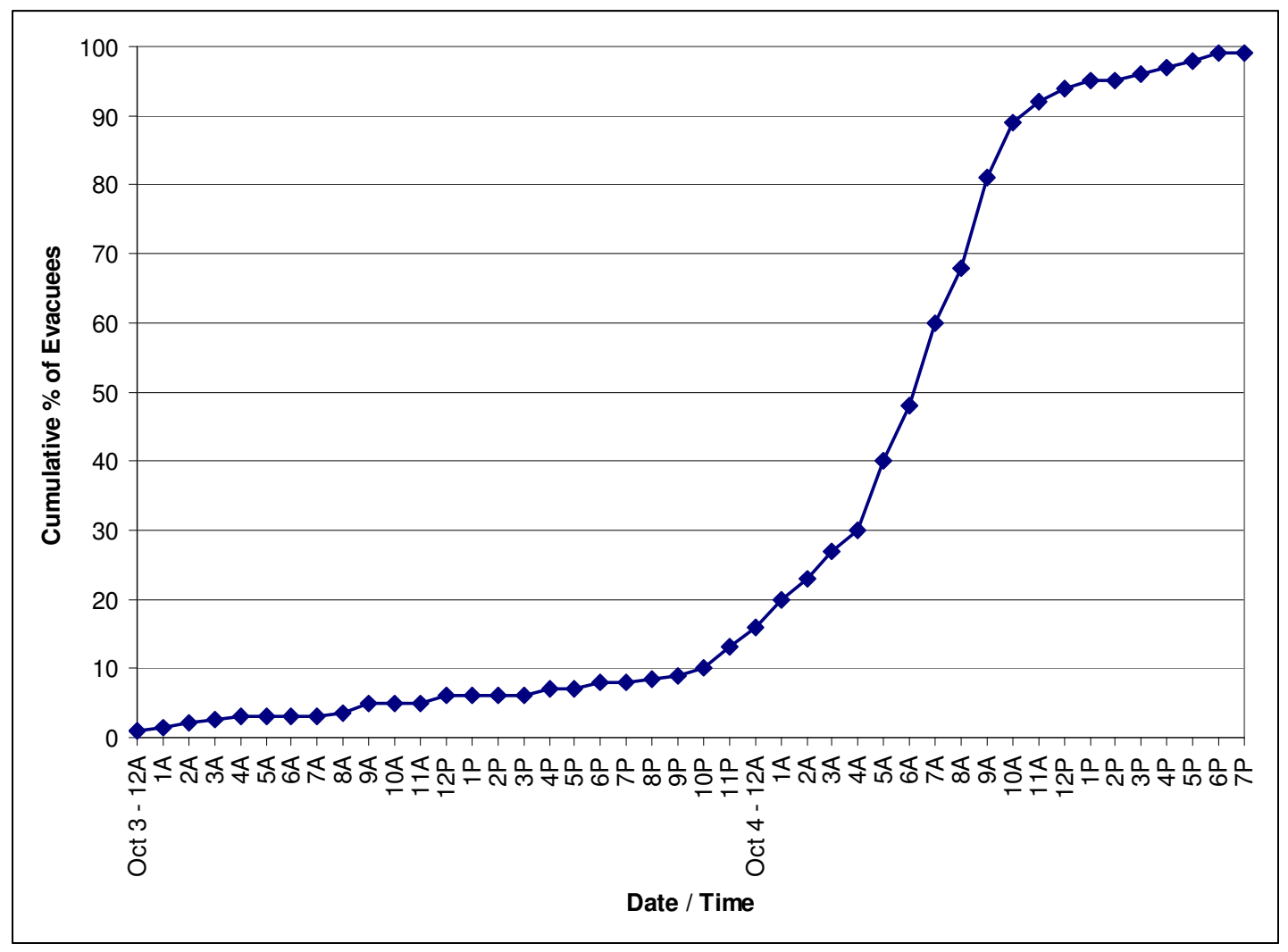

(Source: Adapted from USACE 2006b) 
Figure IV.7. Fast, Medium, \& Slow S-Shaped Evacuation Response Curves

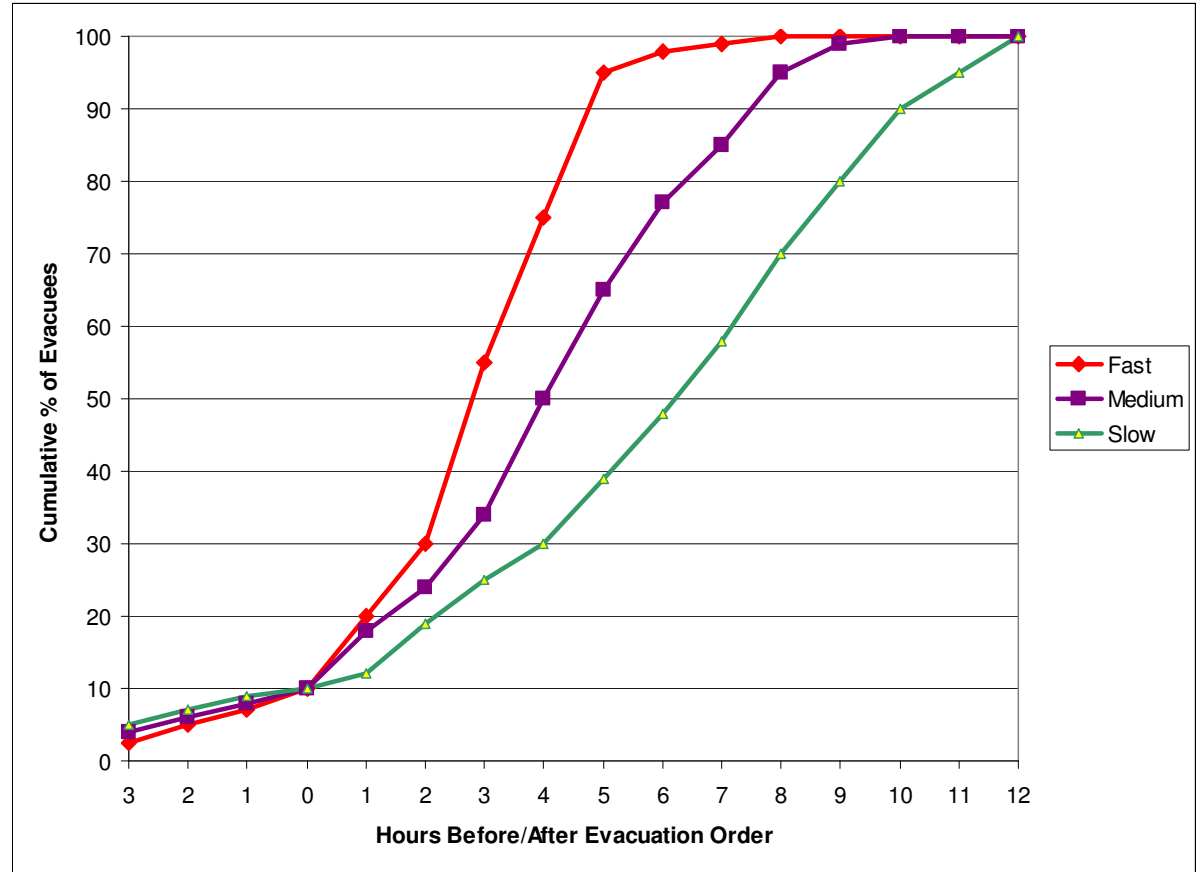

(Source: Adapted from USACE 2006a)

Additionally, other empirical results indicate that these different rates of fast, medium, and slow waiting/evacuating are shown to be dependent upon either household location, or household type. Figure 8 presents evacuation timing results from the Hurricane Jeanne post-storm assessment for the three different Florida aggregated county locations of East Central, Treasure Coast, and Non-Coastal. In this particular instance, although the storm eventually made landfall over the Treasure Coast around 11:00 p.m. on Sept. 25, 2004, East Central generally had a faster rate of evacuation ${ }^{41}$. The NonCoastal location had the slowest rate of evacuation as might be expected. Figure 9 presents fast, medium, and slow evacuation response rates for hurricanes as a function of household type, where households are classified as transient, resident/home, or

\footnotetext{
${ }^{41}$ The 5:00 a.m. 9/24/04 forecast advisory (42 hours before landfall) had the center of the storm making landfall north of the Treasure Coast in the East Central location.
} 
resident/work (Frieser, 2004). Residents with at least one household member working are the most constrained of the three types, and accordingly have the slowest evacuation response time.

Figure IV.8. Hurricane Jeanne Evacuation Response Curve by Household Location

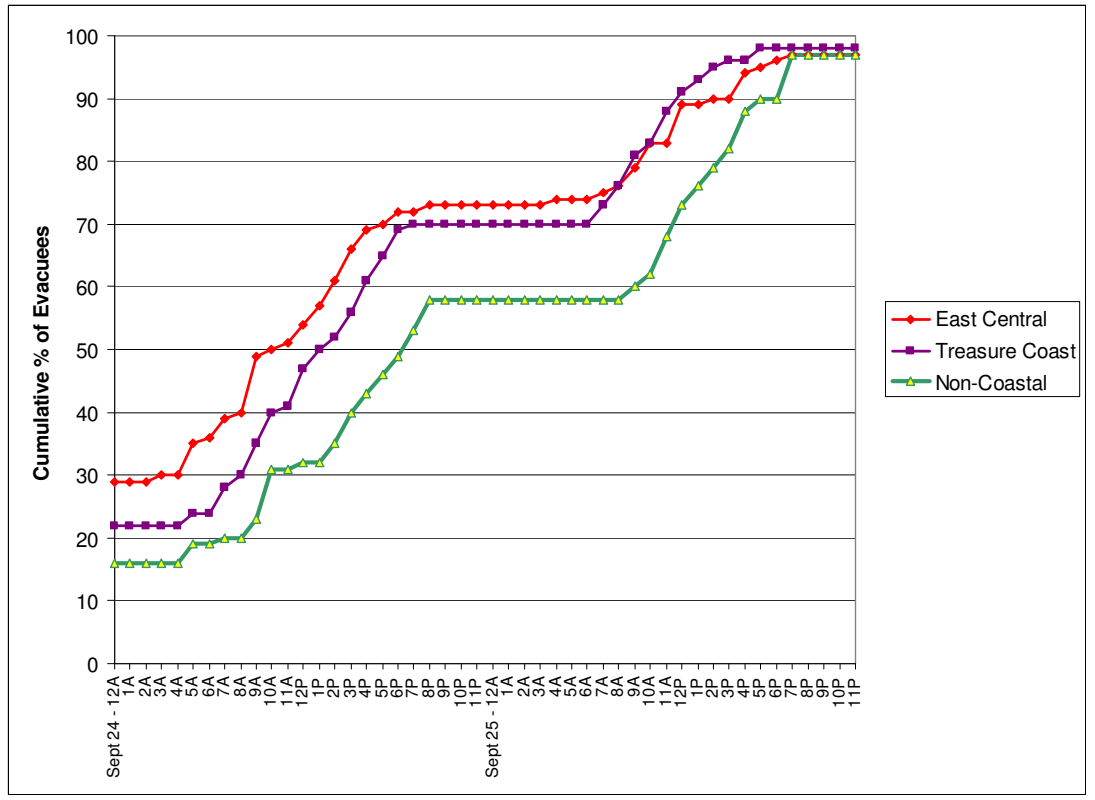

(Source: Adapted from USACE 2006b)

Figure IV.9. Hurricane Evacuation Response Rate by Household Type

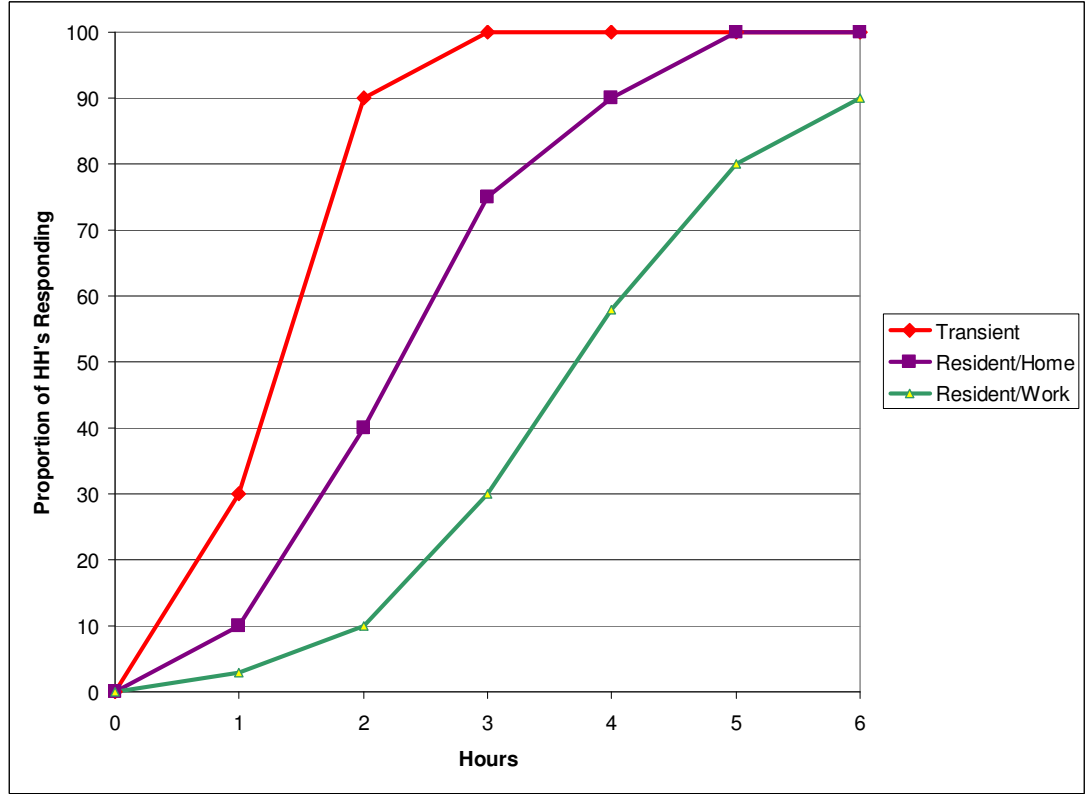

(Source: Adapted from Frieser, 2004) 
Lindell et al. (2005b) further stress location of households (proximity to water sources induces early evacuation), while Fu and Wilmot (2004) further emphasize household type (high risk homes in low-lying areas evacuate earlier) in explaining the heterogeneous timing of evacuations. But, beyond a household's response to an official evacuation order, household location, or household type, there are other factors that emerge from the empirical evacuation literature that provide insight to the variable timing of evacuations. In regard to the starting time of evacuations, Dow and Cutter (pg. 15, 2002) state that the "majority of evacuation trips begin during normal waking hours on the 2 days prior to anticipated landfall", and Lindell et al. (2005a) stress the importance of psychological preparation in initiating the decision to evacuate. $\mathrm{Fu}$ and Wilmot (2004), and Lindell et al. (2005b) highlight heightened rates of evacuation occurring during the daylight hours, and subsequent slowdowns during the night. Lastly, Lindell et al. (2005b) indicate that the steadier is the track of a storm, the earlier will evacuations be induced.

While heterogeneous evacuation behaviors are clearly shown in the existing empirical results, and some insight concerning what is driving these behaviors can also be gained from them, the empirical results do not apply to all general evacuation timing outcomes, nor are they able to sufficiently explain specific evacuation timing outcomes. For example, the evacuation timing graphs from Hurricane Ivan's post-storm assessment illustrate increased levels of evacuation beginning to occur during the nighttime hours, as opposed to slowdowns (Baker, 2005b). Dow and Cutter (2002) are at a loss to explain as to why for Hurricane Floyd (in 1999) 48\% of evacuees left between a 9:00 a.m. to 3:00 p.m. window, with so few leaving before and after this period? Also, as no primary 
factor for the timing of evacuations is identified from the empirical evidence, extrapolating which factor is actually driving evacuation timing for a specific outcome is difficult when interactions between the various factors occur, such as the issuance of evacuation orders during daylight hours for coastal communities (Dow and Cutter, 2002; Lindell et al., 2005b).

It is evident from the above discussion that uncertainty and a household's ability to wait for more information are two qualities that characterize the decision to evacuate when facing the threat of a hurricane. We further assume that the hurricane evacuation decision is irreversible ${ }^{42}$, and therefore the hurricane evacuation decision process is better understood from a dynamic perspective (Dixit and Pindyck, 1994). Our dynamic framework provides the basis for understanding specific evacuation timing outcomes, as they are ultimately driven by an economic cost-benefit rationale. We start by building the intuition for the dynamic perspective through the use of a simple, two-period model of evacuation in the next section.

\section{IV.III. A Two-Period Model of Hurricane Evacuation}

\section{The Model}

We initially frame our household hurricane evacuation decision in a simple twoperiod model, where in each of the two periods evacuation is possible with households in a particular location receiving a forecast of the hurricane intensity at landfall for their

\footnotetext{
${ }^{42}$ For example, mean evacuation distance traveled for Hurricane Ivan was 182 miles (Baker, 2005b) clearly not an easily reversible distance to cover.
} 
location $^{43}$. Let $T$ denote the known ${ }^{44}$ landfall time of the hurricane such that period $1<2$ $<T$, where evacuation in period 1 or 2 is feasible, but evacuation beyond period 2 is not feasible. Furthermore, if evacuation is initiated in either period 1 or 2 , it cannot be reversed.

Let $\theta_{t}$ denote the hurricane landfall intensity forecast for period $t=1,2, T$. The random variable $\theta_{2}$ has a distribution conditional on $\theta_{1}$, while the random variable $\theta_{T}$ has a distribution conditional on $\theta_{2}$; and $\theta_{T}$ is no longer a forecast but rather a realized value. Therefore, the household evacuation decision in period 1 is either to evacuate immediately given $\theta_{1}$, or to wait one period for more information from the expected updated forecast in period 2. Similarly for those households that did not evacuate in period 1, the household evacuation decision in period 2 is either to evacuate immediately given the now realized updated forecast of $\theta_{2}$, or to wait and simply ride out the storm at $T$ based upon the severity of the storm which is a random variable with distribution conditioned by $\theta_{2}$. Since period 2 is the last feasible period to evacuate, waiting to receive information from a further updated forecast beyond period 2 would not matter to the period 2 evacuation decision.

Thus, in both periods 1 and 2 households ultimately decide to either evacuate or wait, and we assume that households base this evacuation decision in each of the $t=1,2$ periods by comparing each period's costs of evacuation, denoted $c_{E V_{(t)}}$, vs. the costs of waiting, denoted $c_{W_{(t)}}$. In either period, $c_{E V_{(t)}}$ are incurred immediately and therefore are

\footnotetext{
${ }^{43}$ For our two-period model we are not incorporating any uncertainty in regard to the track of the storm, and therefore forecasts only contain information regarding the intensity of the storm at landfall. We do allow for track uncertainty in our multi-period model.

${ }^{44}$ Uncertainty in regard to the timing of landfall is also not incorporated into our two-period model.
} 
assumed to be known with certainty. However, $c_{W_{(t)}}$ are a function of how $\theta_{t}$ evolves into $\theta_{t+1}$ for $t=1,2, T$, and given the intrinsic uncertainty of forecasts as presented in section II, $c_{W_{(t)}}$ are consequently uncertain. Therefore, risk neutral, expenditure minimizing households faced with an impending hurricane decide to evacuate in period 1 when $c_{E V_{(1)}}<E_{1}\left[c\left(\theta_{2}\right)_{W} \mid \theta_{1}\right]$, and similarly decide to evacuate in period 2 when $c_{E V_{(2)}}<E_{2}\left[c\left(\theta_{T}\right)_{W} \mid \theta_{2}\right]$

$c_{E V_{(t)}}$ include (but are not limited to) evacuation travel and time costs, direct costs incurred while away (food, lodging, entertainment), lost wages, and the disutility from not being able to protect and/or access one's home (Whitehead, 2003). While some of these immediate evacuation costs, such as lost wages and portions of direct costs, may actually decrease with waiting as $t$ approaches $T$, we assume that certain costs, e.g., the crowdedness of the roads, distance needed to travel for adequate lodging, gasoline scarcity, etc., will increase rapidly enough so as to offset these declining costs. Therefore, we assume that the longer a household waits to evacuate, the more difficult, and hence more expensive the overall evacuation will become such that the costs of evacuating are increasing over time and consequently we have $c_{E V_{(1)}}<c_{E V_{(2)}}$. Indeed, if $c_{E V_{(t)}}$ are not increasing over time, there would be an incentive for households to simply wait until the last possible minute to evacuate. The empirical evidence from the S-shaped evacuation response curves presented in section II do not point to such a last minute evacuation result for all households. 
A household's decision not to evacuate carries the risk of being injured, or even killed, depending on the magnitude of the hurricane and given the hurricane ultimately striking their location. The costs of not evacuating as a function of hurricane intensity therefore include (but are not limited to) the expected loss due to injury, death, and/or personal property damage ${ }^{45}$, as well as the expected utility loss from not being able to maintain a normal level of standard "comforts of home" such as electricity and hot water.

Since in period 1 households wait for the updated forecast, $\theta_{2}$, and the possibility of evacuating during period 2, but in period 2 households wait for the final evolution of the storm, $\theta_{T}$, with no further possibility of evacuating beyond period 2 , the expected costs of waiting in each of these periods will be different. In period 1 , the expected costs of waiting are comprised of two components: the known costs of evacuating in the next period, $c_{E V_{(2)}}$, which we have assumed to be increasing; and the costs of not evacuating, $c\left(\theta_{2}\right)_{N_{-} E V}$ such that:

$$
\begin{aligned}
& E_{1}\left[c\left(\theta_{2}\right)_{W} \mid \theta_{1}\right]=c_{E V_{(2)}} * \operatorname{Pr}\left\{c_{E V_{(2)}}<c\left(\theta_{2}\right)_{N_{-} E V} \mid \theta_{1}\right\} \\
&+E\left[c\left(\theta_{2}\right)_{N_{-} E V} \mid \theta_{1}\right] * \operatorname{Pr}\left\{c_{E V_{(2)}}>c\left(\theta_{2}\right)_{N_{-} E V} \mid \theta_{1}\right\}
\end{aligned}
$$

In period 2, the expected costs of waiting only consist of the expected costs of not evacuating, $E\left[c\left(\theta_{T}\right)_{N_{-} E V} \mid \theta_{2}\right]$, such that:

$$
E_{2}\left[c\left(\theta_{T}\right)_{W} \mid \theta_{2}\right]=\operatorname{Pr}\left[c\left(\theta_{T}\right)_{N_{-} E V} \mid \theta_{2}\right]
$$

\footnotetext{
45 Personal property here represents personal property that a household can take with them during the evacuation such as their automobile.
} 
Importantly, the differences between (23) and (24) highlight the additional insight provided by a dynamic perspective. In period 2, the evacuation decision is static as there is no ability to wait for more information that is actionable. Here, a household weighs the costs of evacuating in period 2 vs. the expected damages at $T$ of not evacuating and makes their cost-minimizing decision accordingly. However, in period 1 the decision is truly dynamic as the household does have the ability to wait for more information that is actionable in period 2, the information is uncertain, and if they decide to evacuate in period 1, this decision cannot be reversed. As can be seen in (23), the household not only weighs the costs of evacuating in period 1 against the expected damages at period 2 of not evacuating, but must also factor into its cost-minimizing decision the known increased costs of evacuating in period 2.

Intuitively, then the value a household ascribes to waiting is that period 2 evacuation may not be optimal due to the intrinsic uncertainty of the forecast, and hence households avoid the regret caused by unnecessary period 1 evacuation. On the other hand, waiting may come at a cost to households if increased evacuation costs are incurred for period 2 evacuations that could have been completed in period 1 at lower evacuation cost levels, or if additional exposure to the costs of not evacuating are incurred in period 2 due to increased evacuation costs that make period 2 evacuation no longer optimal when it would have been optimal if costs had not increased from the period 1 levels. When the regret from evacuating in period 1 is greater than the increased period 2 evacuation costs incurred plus any additional exposure to the costs of not evacuating, a positive option value (OV) for waiting exists. 


\section{Model Solution and Hypothetical Results}

We investigate evacuation decisions in period 1 and period 2 given the potential for a CAT 1, CAT 2, or CAT 3 hurricane making landfall at our location with certainty. Let costs at landfall due to not evacuating for a CAT 2 hurricane be $\$ 450$, increase by $50 \%$ to $\$ 675$ for a CAT 3 hurricane, and decrease by $50 \%$ to $\$ 225$ for a CAT 1 hurricane ${ }^{46}$. The values for losses at landfall due to not evacuating are chosen to be comparable in magnitude to the costs of evacuation from Whitehead (2003), where evacuation costs for CAT 1, 2, and 3 hurricanes are \$211, \$233, and \$273 respectively. Also, let the costs of evacuation increase by $25 \%$ from period 1 to period 2 .

We assume that $\theta_{2}$ does not contain any uncertainty regarding the strength of the hurricane, and therefore perfectly reveals the intensity level of the hurricane at $T$. In this way, the period 2 forecast can be thought of as being close enough to $T$ to allow for a nearly zero intensity forecast error, but far enough away to still allow for safe evacuation ${ }^{47}$. Given the certainty of $\theta_{2}$, households decide to evacuate in period 2, or wait and simply ride out the storm at $T$ by minimizing

$$
\min \left\{c_{E V_{(2)}}, c\left(\theta_{2}\right)_{W}\right\}
$$

Assuming $c_{E V_{(1)}}=\$ 250$ for a CAT 1, 2, or 3 hurricane, and given our assumption that the costs of evacuation increase by $25 \%$ from period 1 to period $2, c_{E V_{(2)}} \approx \$ 310$ for a CAT 1 ,

\footnotetext{
${ }^{46} \mathrm{We}$ have assumed uniform variance in the costs of not evacuating across Cat 1 to CAT 3 hurricanes for purposes of this example, although damage costs (typically property damage) associated with the SSHS are thought to increase exponentially.

${ }^{47}$ Typically there exists a degree of uncertainty surrounding the forecast at the last possible safe evacuation period as shown in Figures 2 and 3. Our assumption is purely for simplification purposes and does not affect the results
} 
2, or 3 hurricane ${ }^{48}$. Therefore, given $\left[c\left(\theta_{T}\right)_{N_{-} E V} \mid \theta_{2}=C A T 1\right]=\$ 225$, $\left[c\left(\theta_{T}\right)_{N_{-} E V} \mid \theta_{2}=C A T 2\right]=\$ 450,\left[c\left(\theta_{T}\right)_{N_{-} E V} \mid \theta_{2}=C A T 3\right]=\$ 675$, and $c_{E V_{(2)}} \approx \$ 310$, from (25) a household will choose to evacuate in period 2 for $\theta_{2}=\operatorname{CAT} 2$ and $\theta_{2}=\operatorname{CAT} 3$, but choose not to evacuate in period 2 for $\theta_{2}=$ CAT 1 .

Let us further assume that $\theta_{1}$ calls for a CAT 2 hurricane making landfall at time $T$ with some inherent uncertainty, and that the inherent uncertainty contained in this period 1 forecast is understood to be with probability $\mathrm{q}=0.34$ that $\theta_{2}=\mathrm{CAT} 2$, with probability $\mathrm{r}=0.33$ that $\theta_{2}=\mathrm{CAT} 3$, and with probability $(1-\mathrm{q}-\mathrm{r})=0.33$ that $\theta_{2}=\mathrm{CAT} 1$. Given this inherent uncertainty of $\theta_{l}$, households must decide to evacuate in period 1 , or wait one period for more information from the expected updated forecast, $\theta_{2}$, and the possibility of evacuating during period 2 by minimizing

$$
\min \left\{c_{E V_{(1)}}, E_{1}\left[c\left(\theta_{2}\right)_{W} \mid \theta_{1}\right]\right\}
$$

where following from (1) we have that

$$
\begin{array}{rl}
E_{1}\left[c\left(\theta_{2}\right)_{W} \mid \theta_{1}\right]=q & * \min \left\{c_{E V_{(2)}}, c\left(\theta_{2}=C A T 2\right)_{N_{-} E V} \mid \theta_{1}=C A T 2\right\} \\
& +r * \min \left\{c_{E V_{(2)}}, c\left(\theta_{2}=C A T 3\right)_{N_{-} E V} \mid \theta_{1}=C A T 2\right\} \\
& +(1-q-r) * \min \left\{c_{E V_{(2)}}, c\left(\theta_{2}=C A T 1\right)_{N_{-} E V} \mid \theta_{1}=C A T 2\right\}
\end{array}
$$

From period 2 we know that the $\min \left\{c_{E V_{(2)}}, c\left(\theta_{T}\right)_{N_{-} E V} \mid \theta_{2}=C A T 1\right\}=\$ 225$, $\min \left\{c_{E V_{(2)}}, c\left(\theta_{T}\right)_{N_{-} E V} \mid \theta_{2}=C A T 2\right\}=\$ 310$, and $\min \left\{c_{E V_{(2)}}, c\left(\theta_{T}\right)_{N_{-} E V} \mid \theta_{2}=C A T 3\right\}=\$ 310$.

\footnotetext{
${ }^{48}$ Costs of evacuation are assumed to be the same for CAT 1, 2, and 3 hurricanes in our two-period model, and are comparable to the range of the evacuation costs for CAT 1,2, and 3 hurricanes from Whitehead (2003). The costs will vary by CAT level in our multi-period model.
} 
Since $\left(\theta_{T} \mid \theta_{2}\right)$ and $\left(\theta_{2} \mid \theta_{1}\right)$ we can substitute these min values from period 2 into (27) to obtain $E_{1}\left[c\left(\theta_{2}\right)_{W} \mid \theta_{1}\right]=\{[.33 * \$ 225]+[.34 * \$ 310]+[.33 * \$ 310]\}=\$ 284$ and solve $(26)$ where $c_{E V_{(1)}}=\$ 250$. Therefore, in period 1 given that $\theta_{1}$ calls for a CAT 2 hurricane making landfall at time $T$ with probability $\mathrm{q}=0.34$ that $\theta_{2}=\mathrm{CAT} 2, \mathrm{r}=0.33$ that $\theta_{2}=\mathrm{CAT} 3$, and $(1-\mathrm{q}-\mathrm{r})=0.33$ that $\theta_{2}=\mathrm{CAT} 1$, that $c_{E V_{(1)}}=\$ 250$ increasing by $25 \%$ to $c_{E V_{(2)}} \approx \$ 310$, and that $\left[c\left(\theta_{T}\right)_{N_{-} E V} \mid \theta_{2}=C A T 1\right]=\$ 225,\left[c\left(\theta_{T}\right)_{N_{-} E V} \mid \theta_{2}=C A T 2\right]=\$ 450$, $\left[c\left(\theta_{T}\right)_{N_{-} E V} \mid \theta_{2}=C A T 3\right]=\$ 675$, it is rational for a household to evacuate in period 1.

Viewing the decision to evacuate in period 1 from a static now or never perspective would also tell us that evacuation in period 1 is optimal when $c_{E V_{(1)}}=\$ 250$. This is because from a static perspective, a household decides to evacuate in period 1 by minimizing

$$
\min \left\{c_{E V_{(1)}}, E_{1}\left[c\left(\theta_{2}\right)_{N_{-} E V} \mid \theta_{1}\right]\right\}
$$

where $E_{1}\left[c\left(\theta_{2}\right)_{N_{-} E V} \mid \theta_{1}\right]=\{[.33 * \$ 225]+[.34 * \$ 450]+[.33 * \$ 675]\}=\$ 450$. Figure 10 therefore shows evacuation in period 1 being optimal up to $c_{E V_{(1)}}=\$ 450$ when the evacuation decision in period 1 is framed from a "now or never" static viewpoint.

However, since a household has the ability to wait for more information that is actionable in period 2, a dynamic view of evacuation behavior in period 1 is more appropriate. As $c_{E V_{(2)}}=(1.25) * c_{E V_{(1)}}$, and given our model assumptions, we can solve (26) for various levels of $c_{E V_{(1)}}$ to contrast the difference between viewing the period 1 
household evacuation decision from a dynamic vs. static perspective. Figure 11 indicates that for $c_{E V_{(1)}} \geq \$ 387$ it is not optimal to evacuate in period 1 , but rather it is optimal to wait for the updated period 2 forecast. Thereby, Figure 11 illustrates that from a dynamic perspective $1^{\text {st }}$ period waiting is optimal for certain levels of evacuation costs, specifically $\$ 387$ to $\$ 450$, where $1^{\text {st }}$ period evacuation was optimal from a static perspective.

Figure IV.10. A Static Perspective of Period 1 Evacuation

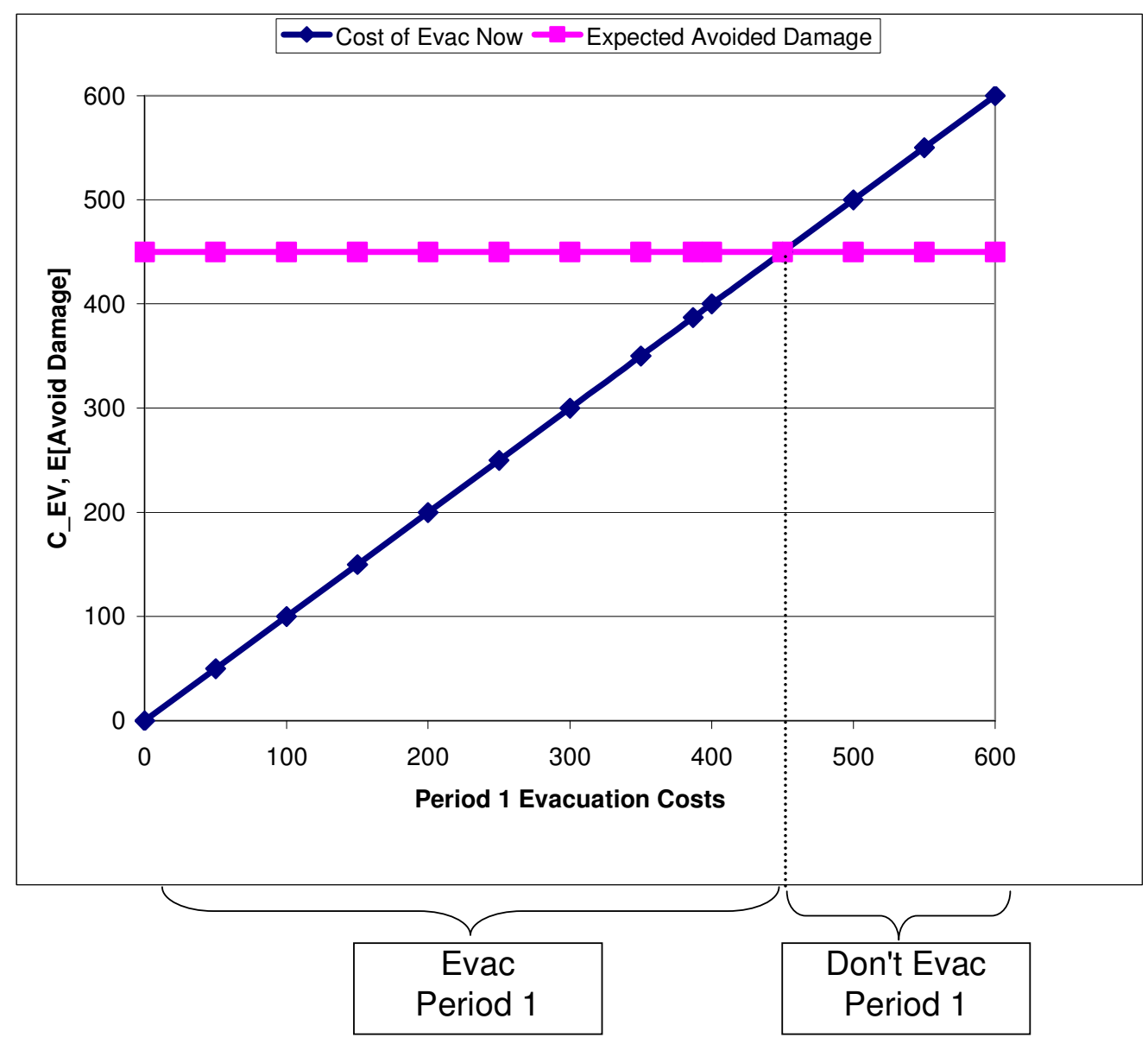


Figure IV.11. Dynamic vs. Static Perspective of Period 1 Evacuation

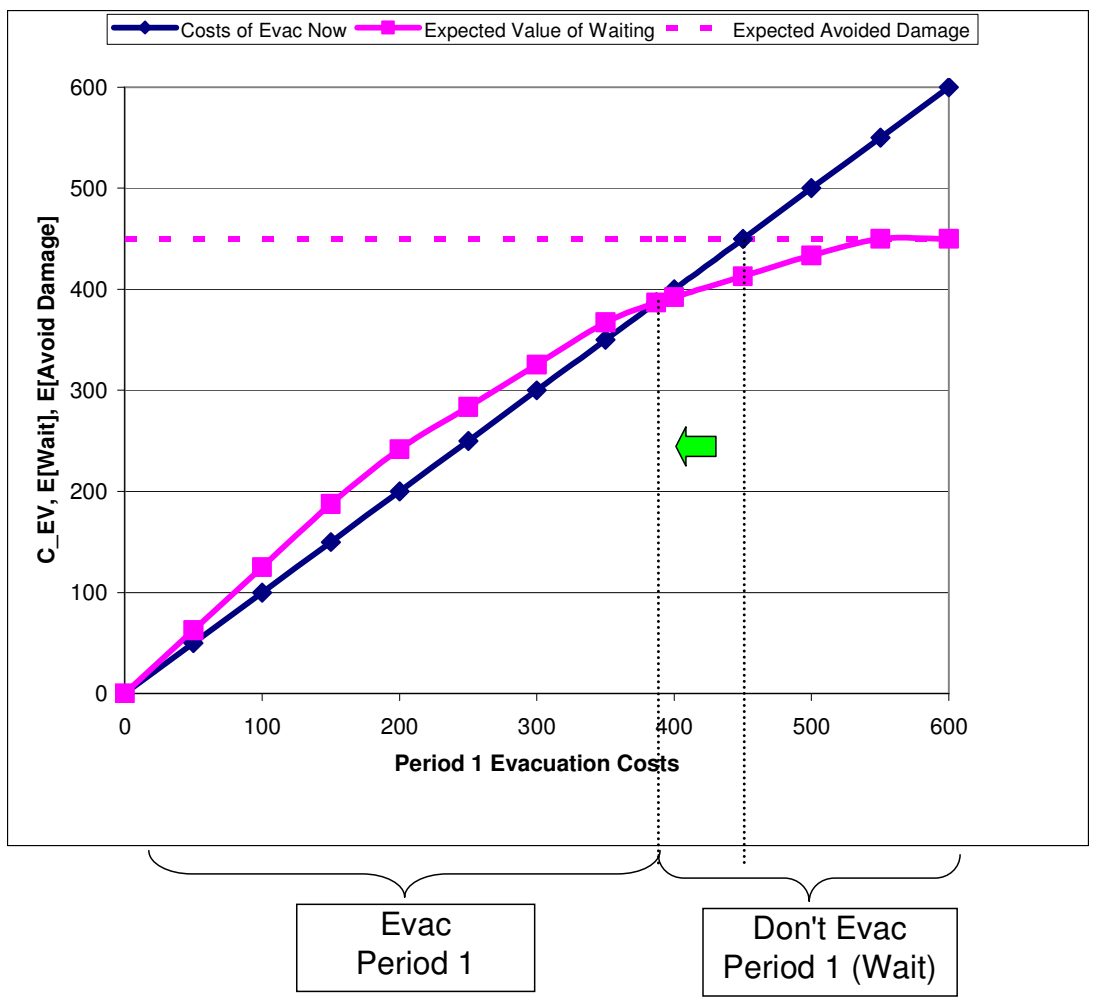

The dynamic results indicate that when relatively high levels of period 1 evacuation costs, [\$387 - \$450], are combined with the assumed uncertainty of $\theta_{1}$, the potential regret for evacuating in period 1 outweighs the additional incurred costs of waiting such that waiting for $\theta_{2}$ is the optimal period 1 choice, and therefore a positive $\mathrm{OV}$ for waiting exists. The increasing positive $\mathrm{OV}$ to waiting for the period 2 forecast is represented by the increasing difference between the expected value of waiting and cost of evacuating now curves of Figure 11 for $c_{E V_{(1)}} \geq \$ 387$. So even if there is a positive net benefit to evacuating now from a static perspective, e.g., when $c_{E V_{(1)}}=\$ 400$ a positive net benefit to evacuating now $\$ 450$ - $\$ 400>\$ 0$ exists, it is still rational for households to wait for the revised forecast due to the positive OV revealed from a dynamic decision 
framework. The result that it is rational for households to wait in this situation is lost if not viewed from a dynamic perspective, leaving open the possibility of overestimation of period 1 evacuation response rates. Finally, it should be clear that by lowering period 1 evacuation costs below $\$ 387$, the regret for evacuating during period 1 is reduced and hence so is the value of waiting, leading to more period 1 evacuation.

\section{Policy Implications}

We can also use this two-period setup to better understand how the implementation of specific hurricane related policies will affect the levels of period 1 evacuation. For example, assume that more strict building codes are put into place in order to reduce the costs of not evacuating (i.e., expected loss due to injury, etc.) for all three hurricane CAT levels. Let us further assume that given this structural mitigation effort, the costs at landfall due to not evacuating for a CAT 2 hurricane are reduced by $33 \%$ to $\$ 300$, while still increasing by $50 \%$ to $\$ 450$ for a CAT 3 hurricane, and still decreasing by $50 \%$ to $\$ 150$ for a CAT 1 hurricane. Figure 12 shows that even from a static perspective less immediate period 1 evacuation should be affected, as the expected avoided damage curve shifts downward to $\$ 300$, and therefore not evacuating becomes optimal for lower levels of period 1 evacuation costs.

However, the dynamic perspective is the more correct way of framing the evacuation decision, and Figure 13 indicates even lower levels of period 1 evacuation than the static view of Figure 12 predicts. This result is being driven by two separate effects caused by the structural mitigation effort: 1) lower costs of not evacuating, but also 2) an assumed slower rate of period 1 to period 2 cost of evacuation increases (20\% 
vs. 25\%) for all CAT levels due to less overall people evacuating, which cannot be captured in a static now or never view.

Figure IV.12. A Static Perspective of Period 1 Evacuation Given Structural Mitigation

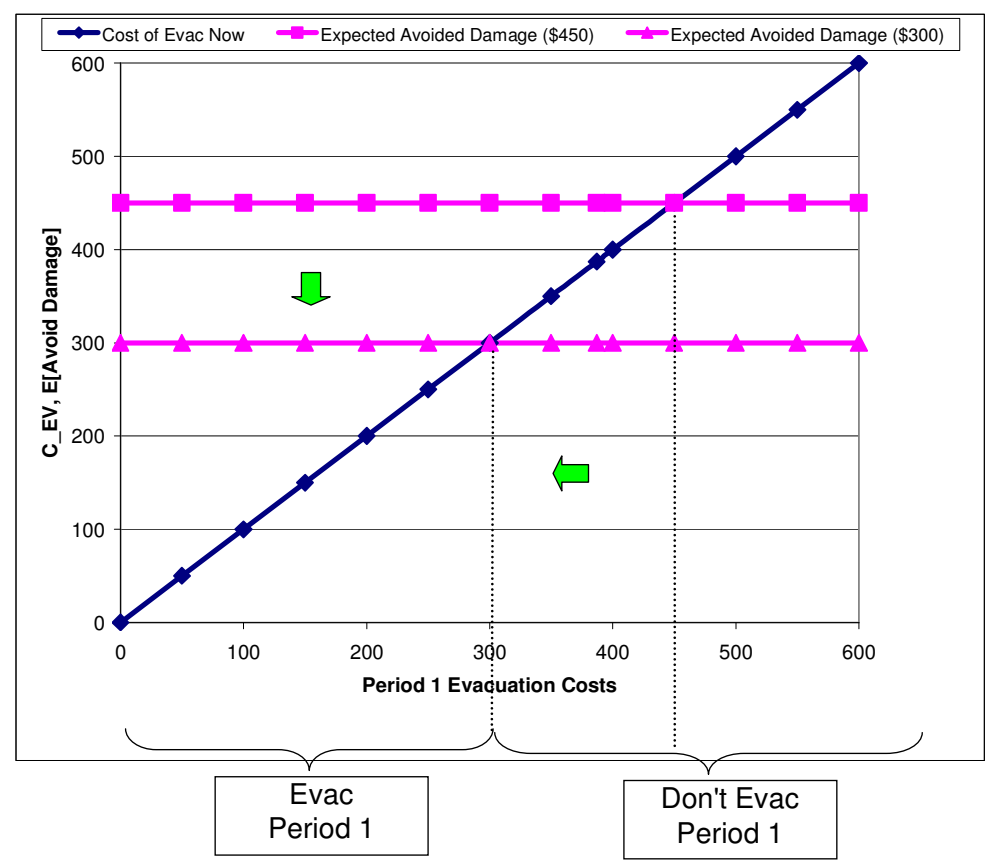

Figure IV.13. A Dynamic Perspective of Period 1 Evacuation Given Structural Mitigation

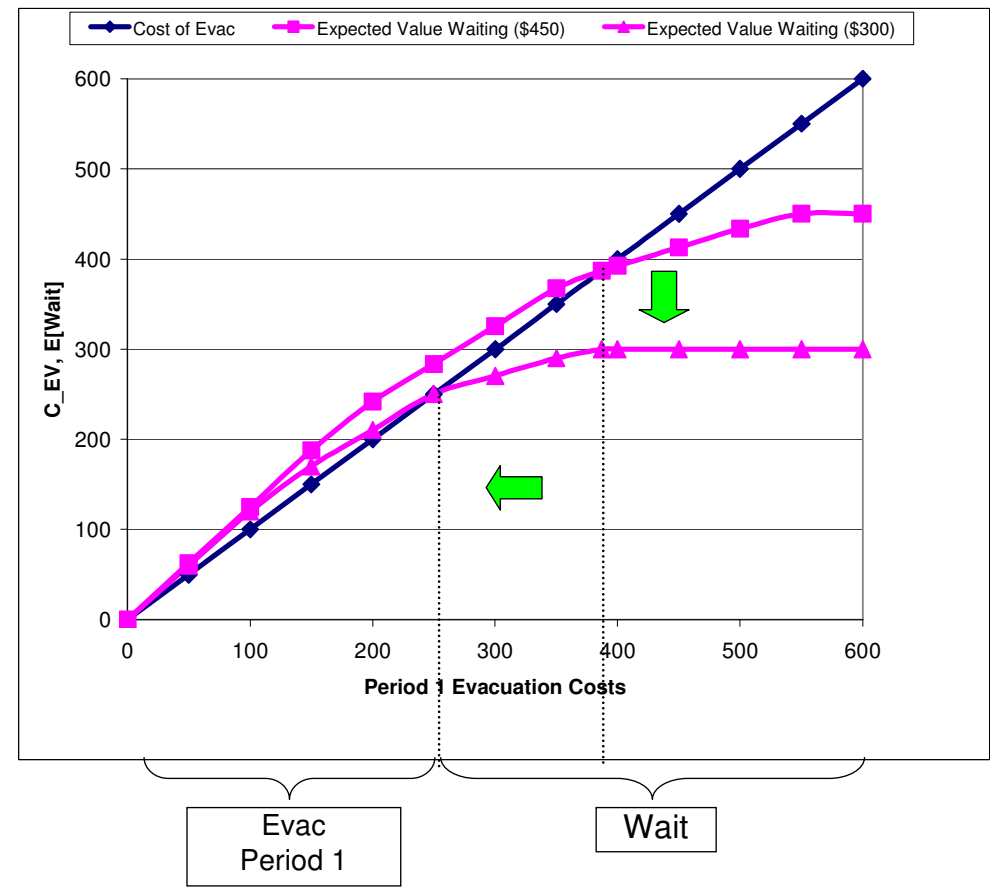


A similar static vs. dynamic period 1 evacuation outcome can be shown for the scenario where less uncertainty in the forecasts is achieved for stronger storms. Let the inherent uncertainty of $\theta_{1}$ be such that the probability of Cat 3 storm increases from $\mathrm{r}=$ 0.33 to $r=0.50$, while decreasing $q=0.25$ and $(1-q-r)=0.25$. Figure 14 shows that even from a static perspective more immediate period 1 evacuation should be affected, as the expected avoided damage curve shifts upward to $\$ 506$, and therefore evacuating becomes optimal for higher levels of period 1 evacuation costs. From a dynamic perspective, Figure 15 indicates slightly less significant increases to period 1 evacuation than the static view of Figure 14 predicts. This result is being driven by two separate effects caused by the increased r value: 1) overall higher expected costs of not evacuating, but also 2) an assumed higher rate of period 1 to period 2 cost of evacuation increases for CAT 3 storms (25\% to $30 \%$ ) due to more overall people evacuating, combined with an assumed lower rate of period 1 to period 2 cost of evacuation increases for CAT 1 and 2 storms $(25 \%$ to $20 \%)$ due to less overall people evacuating, which again would not be captured in a static now or never view.

Still, differences between the static and dynamic frameworks will for some questions be more substantive. For example, assume the structural mitigation efforts are focused on minimizing damages from the more frequent, but relatively less destructive Cat 1 and Cat 2 hurricanes ${ }^{49}$. In this scenario, we assume that the costs at landfall due to not evacuating for a CAT 2 hurricane remain at $\$ 450$, but now increase by $75 \%$ to $\$ 788$ for a CAT 3 hurricane, and decrease by $75 \%$ to $\$ 113$ for a CAT 1 hurricane. Here,

\footnotetext{
${ }^{49}$ CAT 1 hurricanes represent $40 \%$, and CAT 2 represent $26 \%$, of the total hurricanes with a direct hit on the United States Mainland (Blake et al., 2005).
} 
results from a static perspective will show no changes to period 1 evacuation levels occurring because the expected avoided damages do not change, as $E_{1}\left[c\left(\theta_{2}\right)_{N_{-} E V} \mid \theta_{1}\right]=\{[.33 * \$ 113]+[.34 * \$ 450]+[.33 * \$ 788]\}=\$ 450$. But analyzing this policy from a dynamic viewpoint will result in lower levels of period 1 evacuation being predicted due to the downward shift of the expected value of waiting curve as shown in Figure 16. Here, more waiting occurs as the chances of regretting period 1 evacuation increase due to the lower personal damage costs of not evacuating for CAT 1 storms, $\$ 225$ vs. \$113.

Figure IV.14. A Static Perspective of Period 1 Evacuation Given Higher Probability of CAT 3

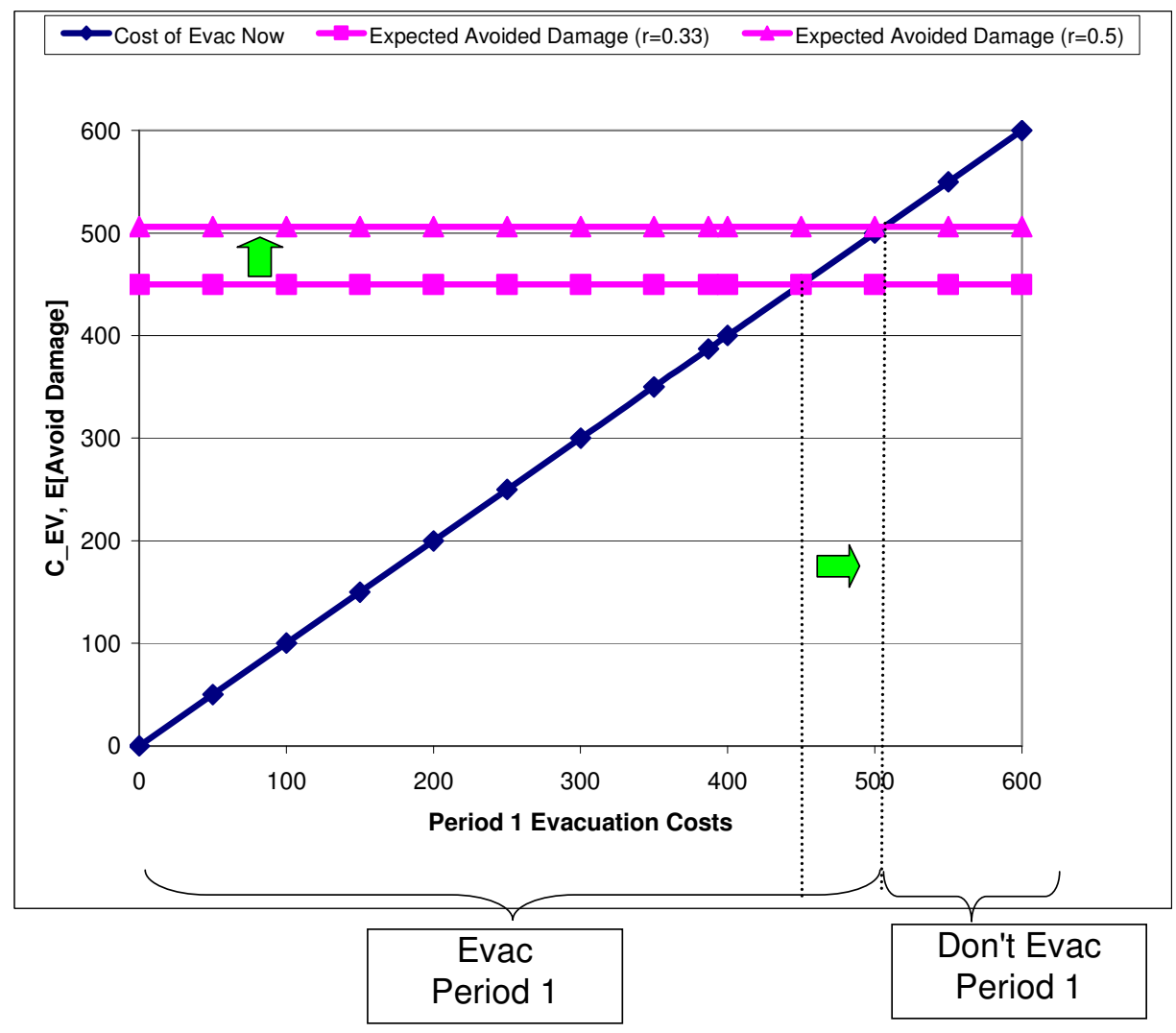


Figure IV.15. A Dynamic Perspective of Period 1 Evacuation Given Higher Probability of CAT 3

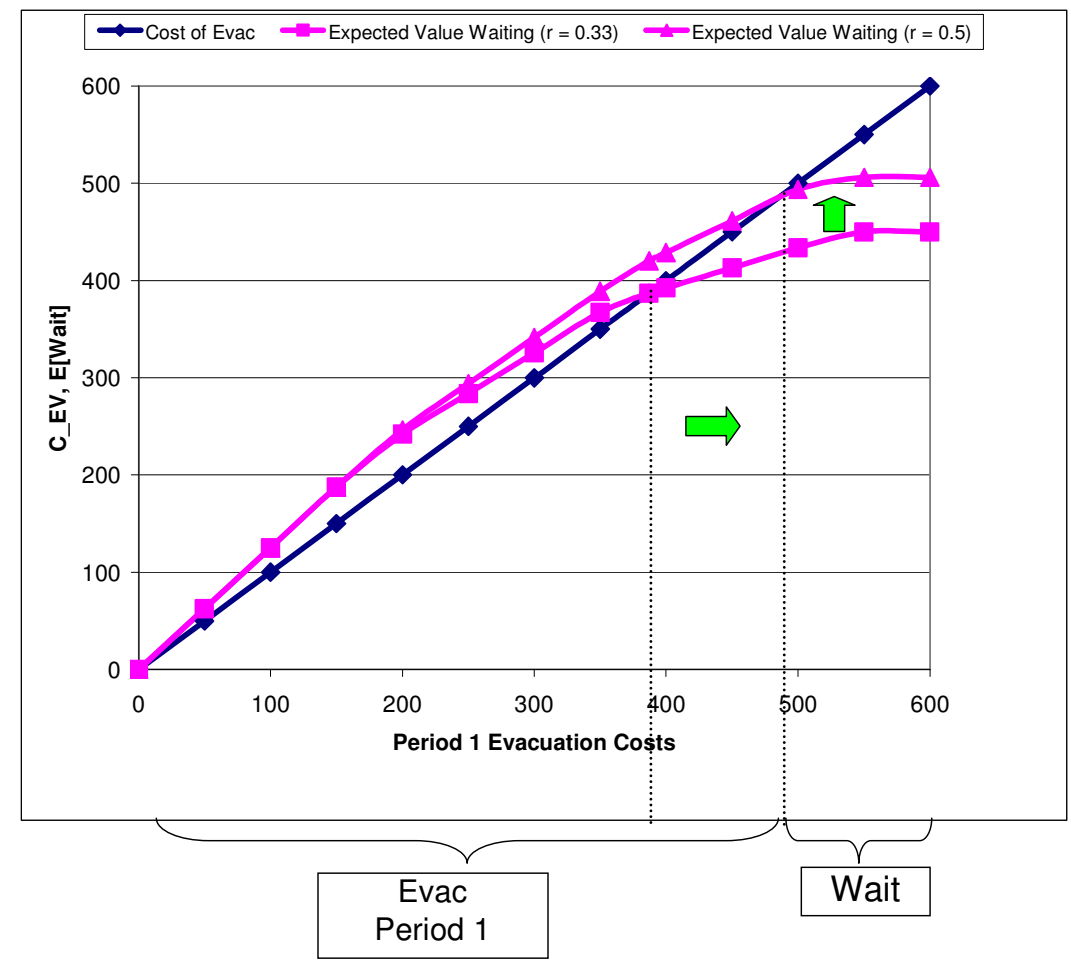

In yet other examples, the static framework may even yield responses that have the wrong sign. For example, assume an "evacuation rebate" is given after the storm to those households that evacuated for up to $10 \%$ of evacuation costs. In this way, period 1 costs are effectively reduced by $10 \%$, which causes a downward pivot of the realized period 1 evacuation cost curve as shown in Figure 17. From a static viewpoint, more period 1 evacuation would be expected. However, the dynamic perspective indicates a different result. From this dynamic perspective, while period 1 evacuation would slightly increase vs. the earlier dynamic results presented in Figure 11, the increase would not be as significant as the static viewpoint seems to indicate, and clearly has waiting being optimal for costs levels where evacuation in period 1 is optimal from the static results. 
Figure IV.16. Dynamic View of Period 1Evac Given Mitigation Focus on Minor Hurricanes

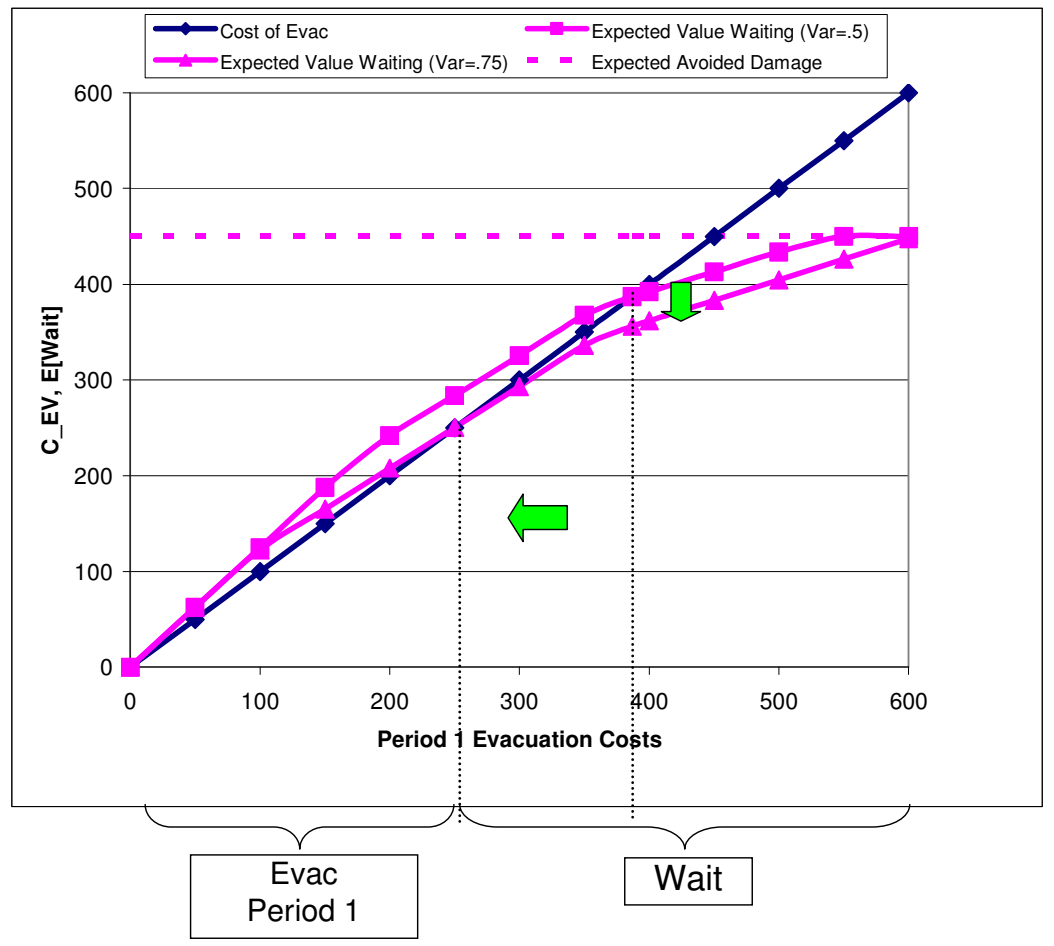

Figure IV.17. A Dynamic Perspective of Period 1 Evacuation Given Evacuation Rebate

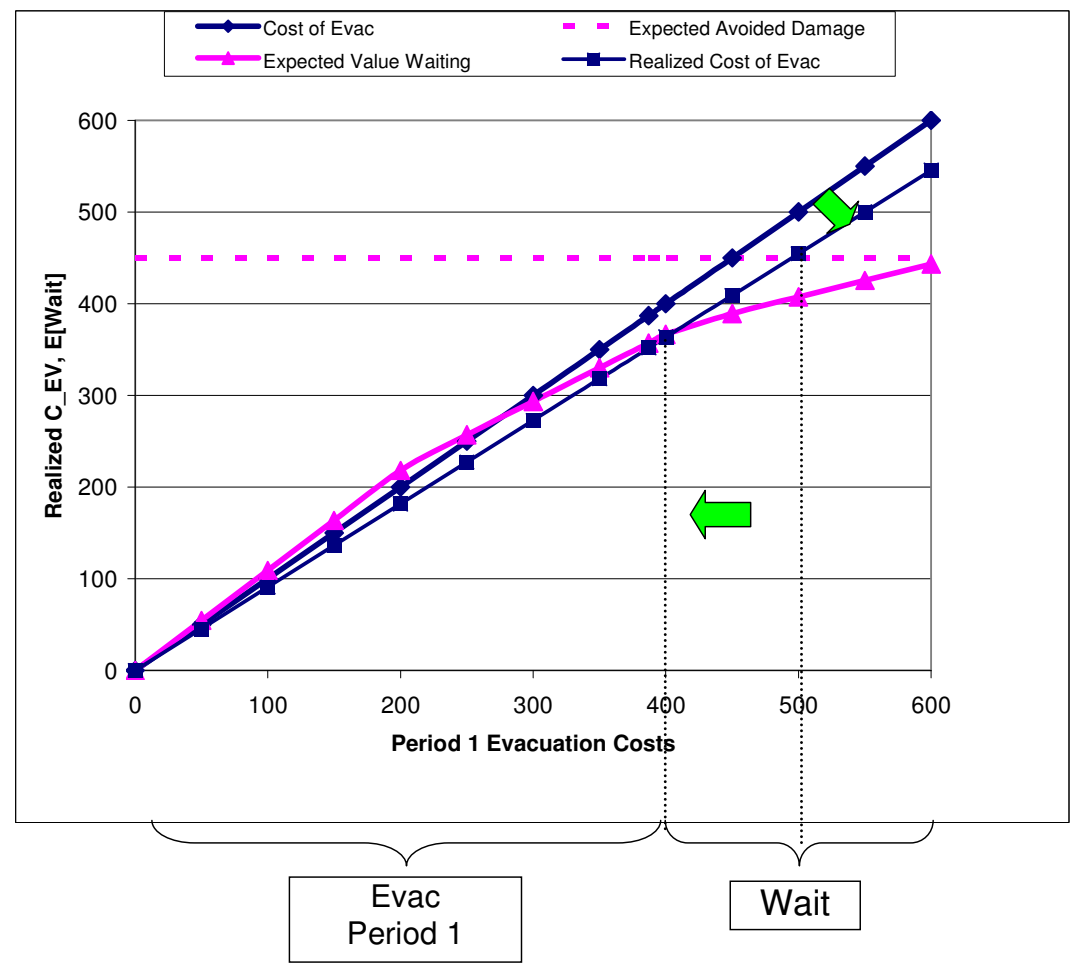


Thus far, our two-period analysis has derived results based upon households with homogeneous period 1 evacuation costs, $c_{E V_{(1)}}=\$ 250$, and $\theta_{1}$ calling for a CAT 2 hurricane making landfall at time $T$ with probability $\mathrm{q}=0.34$ that $\theta_{2}=\mathrm{CAT} 2, \mathrm{r}=0.33$ that $\theta_{2}=\mathrm{CAT} 3$, and (1-q-r) $=0.33$ that $\theta_{2}=\mathrm{CAT} 1$. However, in our two-period model we can also analyze evacuation behavior between a high cost of evacuation household with $c_{E V_{(1)}}=\$ 400$, and a low cost of evacuation household with $c_{E V_{(1)}}=\$ 200$. Furthermore we can do this for different period 1 forecasts such that: when $\theta_{1}$ calls for a CAT 1 hurricane making landfall at time $T$, the inherent uncertainty contained in this period 1 forecast is understood to be with probability $\mathrm{q}=0.4$ that $\theta_{2}=\mathrm{CAT} 2$, with probability $\mathrm{r}=0.2$ that $\theta_{2}=\mathrm{CAT} 3$, and with probability $(1-\mathrm{q}-\mathrm{r})=0.4$ that $\theta_{2}=\mathrm{CAT} 1$; and when $\theta_{1}$ calls for a CAT 3 hurricane making landfall at time $T$, the inherent uncertainty contained in this period 1 forecast is understood to be with probability $\mathrm{q}=0.4$ that $\theta_{2}=\mathrm{CAT} 2$, with probability $\mathrm{r}=0.4$ that $\theta_{2}=\mathrm{CAT} 3$, and with probability $(1-\mathrm{q}-\mathrm{r})=0.2$ that $\theta_{2}=$ CAT 1 .

Still assuming that evacuation costs increase by $25 \%$ from period 1 to period 2 , that $\theta_{2}$ does not contain any uncertainty regarding the strength of the hurricane, and that $\left[c\left(\theta_{T}\right)_{N_{-} E V} \mid \theta_{2}=C A T 1\right]=\$ 225, \quad\left[c\left(\theta_{T}\right)_{N_{-} E V} \mid \theta_{2}=C A T 2\right]=\$ 450, \quad \&$ $\left[c\left(\theta_{T}\right)_{N_{-} E V} \mid \theta_{2}=C A T 3\right]=\$ 675$, we can again solve for the optimal period 2 and period 1 evacuation or wait decisions for both the high evacuation cost and low evacuation cost household types following from (25) and (26) respectively. Figure 18 shows the results by period for the two household evacuation cost types where clearly different 
evacuating and waiting results emerge depending on the household evacuation cost type. In period 2, while low cost types would evacuate for $\theta_{2}=$ CAT 2 , high cost types would only evacuate given $\theta_{2}=$ CAT 3 . In period 1 , the difference is more significant given the uncertainty in $\theta_{1}$ as low cost types would evacuate for $\theta_{1}=\mathrm{CAT} 1$, while again high cost types would only evacuate given $\theta_{1}=$ CAT 3 .

Figure IV.18. Optimal Evacuation by Evacuation Cost Type

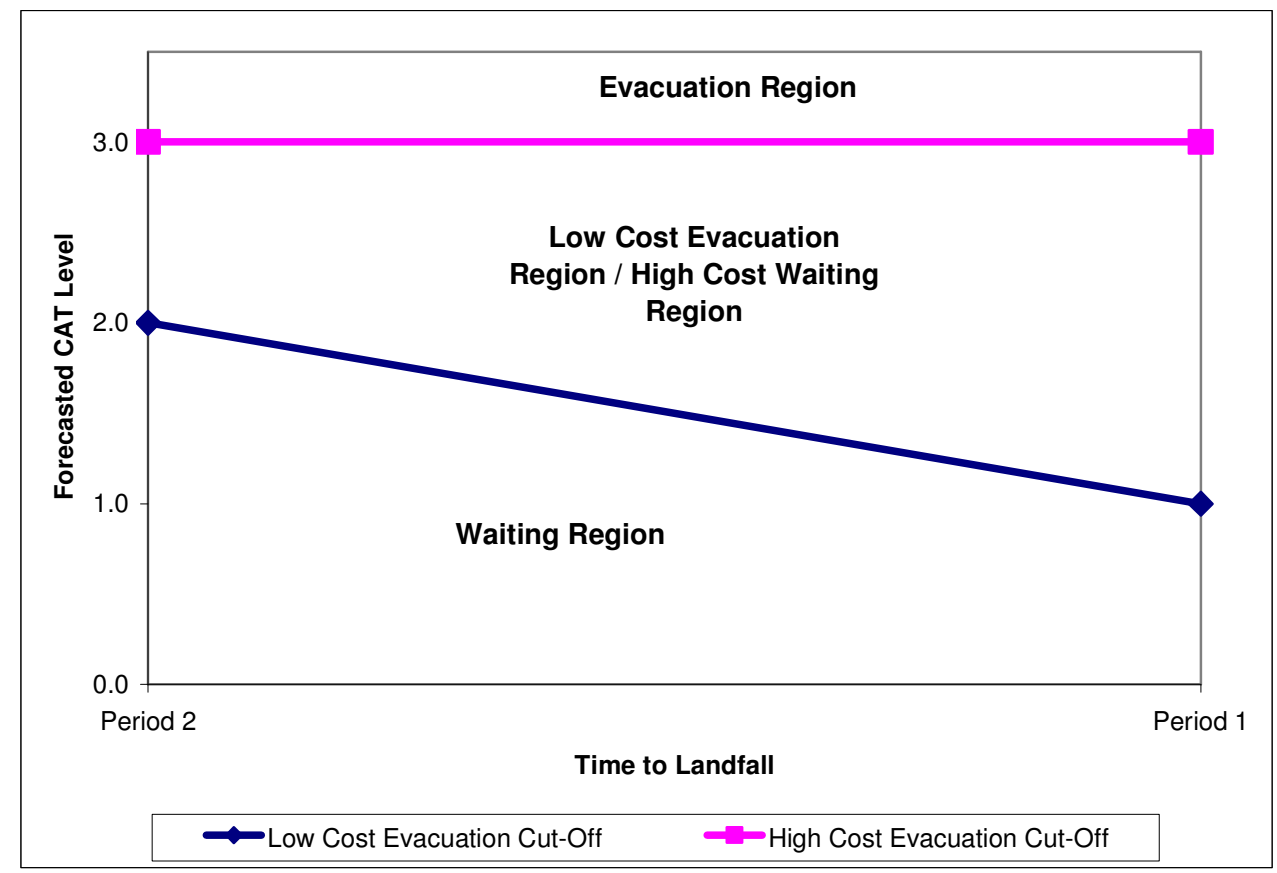

Now let us assume that the government would like to try to make evacuation decisions more equitable between high cost and low cost of evacuation households, but has a hard time distinguishing household evacuation cost type, and therefore lowers the costs of evacuation by $\$ 50$ per household regardless of cost type. Following from our results from Figure 17 where lower evacuation costs may lead to more waiting for an average household, we further assume in this scenario that evacuation costs increase by $20 \%$ from period 1 to period 2 as opposed to the original $25 \%$. Figure 19, illustrates the 
results of this implemented policy where the evacuation region for high cost types has expanded to nearly equaling the low cost type evacuation region prior to the policy. However, the low cost evacuation region also expands in period 2 so divergent evacuation and waiting regions still exist between the household types. The number of injuries avoided or lives saved due to the expanded evacuation regions could be used to determine whether the benefits of implementing this type of policy outweigh the costs.

Figure IV.19. Optimal Evac by Evacuation Cost Type Given $\$ 50$ Evacuation Cost Reduction

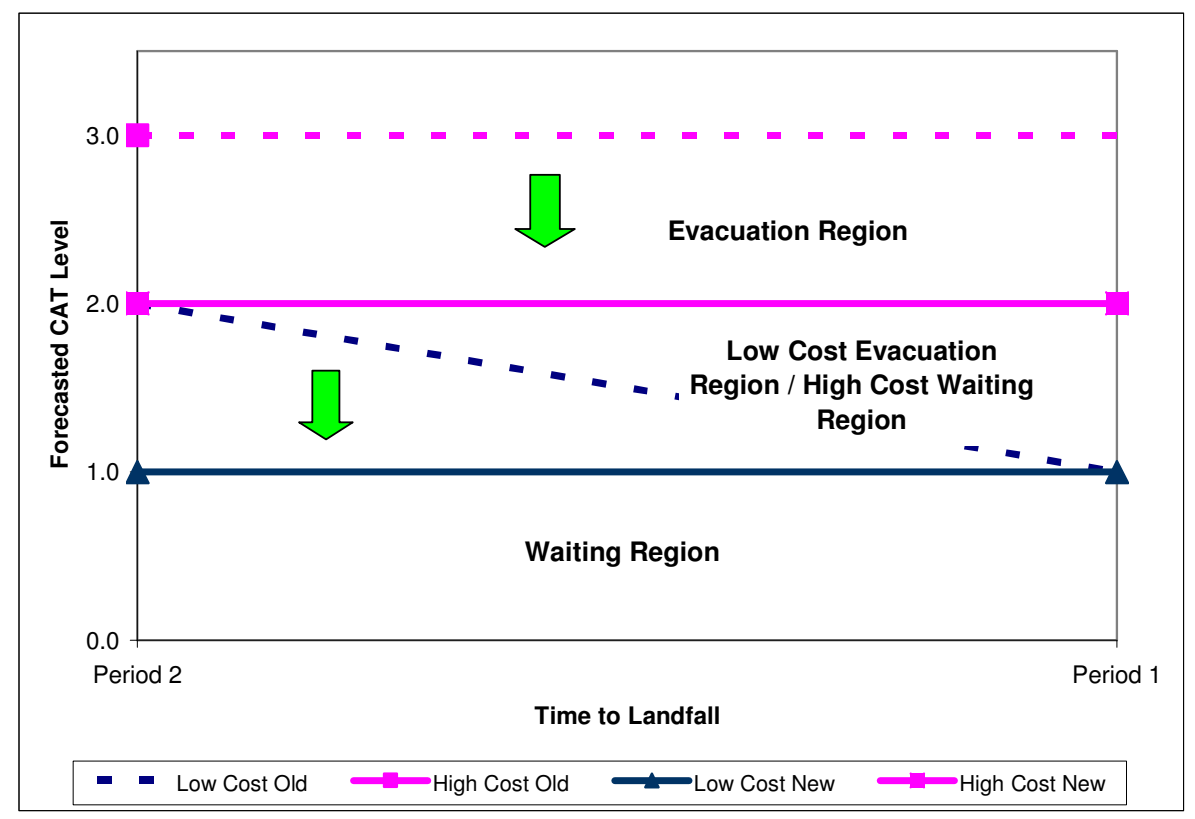

Finally, let us further assume again that due to a structural mitigation effort, the costs at landfall due to not evacuating for a CAT 2 hurricane are reduced by $33 \%$ to $\$ 300$, thereby still increasing by $50 \%$ to $\$ 450$ for a CAT 3 hurricane, and still decreasing by $50 \%$ to $\$ 150$ for a CAT 1 hurricane. In order to achieve the same expanded evacuation region by household type that are achieved in Figure 19 from reducing evacuation costs by $\$ 50$ per household, they now need to be reduced by $\$ 150$ for the high cost type and by $\$ 75$ for the low cost type. Clearly the improved structural mitigation requires a larger 
outlay per household by the government to offset the lower costs of not evacuating and therefore still induce evacuation in either period.

\section{Understanding Heterogeneous Evacuation Behavior}

Results from a dynamic model are not only capable of illustrating the evacuation timing implications of implementing hurricane related policies, but also can provide additional clarity to some of the existing heterogeneous evacuation timing empirical results from Section II. From (23), we have shown that households deciding from a dynamic perspective to evacuate or wait in period 1 must also factor into their cost minimizing decision the known increased costs of evacuating in period 2 , which are not factored into a household's period 1 decision to evacuate or wait from a static perspective. We can therefore use different rates of cost of evacuation increases between period 1 and period 2 in an attempt to better understand some of the existing heterogeneous evacuation timing empirical results such as the different rates of fast, medium, and slow evacuations by household type.

Our dynamic results from Figure 11 assumed a $25 \%$ increase in the costs of evacuation between periods 1 and 2 and indicated that for $c_{E V_{(1)}} \geq \$ 387$ it is not optimal to evacuate in period 1, but rather that it is optimal to wait for the updated period 2 forecast. Figure 20 illustrates how the amount of period 1 waiting increases given that the costs of evacuation between periods 1 and 2 increase only by $10 \%$ as opposed to $25 \%$. In this scenario, the costs of waiting have decreased while the value of waiting has not changed, leading to more overall period 1 waiting. 
We interpret higher levels of period 1 waiting (e.g., as shown by Figure 20) as downward shifts in the traditional s-shaped evacuation response curves (slower evacuation rates), and higher levels of period 1 evacuating as upward shifts in the traditional s-shaped evacuation response curves (faster evacuation rates). In section II, Figure 9 presented fast, medium, and slow evacuation response rates for transient, resident/home, or resident/work households respectively. We combine these interpretations for a better understanding of the different evacuation rates of transients and residents.

Figure IV.20. Optimal Waiting when Evacuation Costs Increase by 10\% vs. 25\%

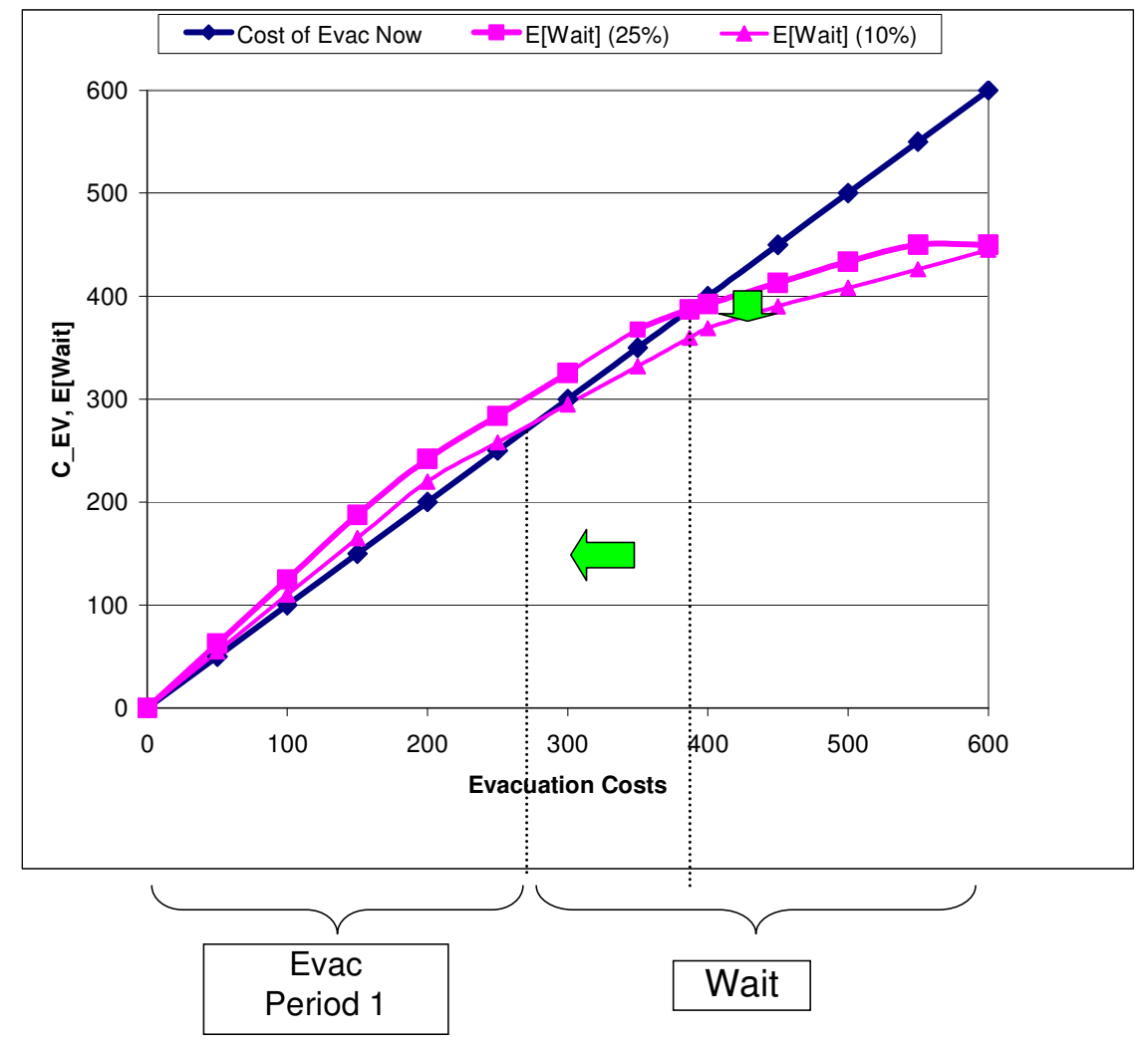

Again, evacuation costs are comprised of travel and time costs, direct costs incurred while away (food, lodging, entertainment), and lost wages, and while some of these immediate evacuation costs may actually decrease with waiting, we have assumed 
that the costs of evacuating are increasing over time. Given this, the rate of increase for transients' evacuation costs from one period to the next will be significant due to their limited options of evacuating, e.g., catching an earlier flight, leading to higher levels of period 1 evacuation, and hence fast evacuation response curves. For non-working residents, while evacuation costs from one period to the next will not increase as significantly as transients, their overall increasing evacuation costs are not offset as much by the time decreasing evacuation cost of lost wages, leading to medium evacuation response rates. The increase in evacuation costs for working residents will be at the lowest rate due to the offset caused by the possibility of lost wages, leading to the slow evacuation response rates.

Baker (2002) found that when those that have evacuated are asked what they would do differently in the future, most respondents said they would still evacuate, only next time it would be earlier. This finding indicates that the more appropriate research question moving forward is not who is going to evacuate, but rather when they will evacuate. Results from our simple, two-period model of evacuation show the additional clarity that can be obtained in regard to when a household will evacuate, by appropriately framing the evacuation decision from a dynamic perspective.

\section{IV.IV. A Multi-Period Model of Evacuation}

While our two-period model of evacuation from Section III is able to provide the intuition behind evacuation decision making in a dynamic framework, it does not represent a realistic evacuation decision situation. Therefore, we extend our two-period 
model to a realistic multi-period setup where households have the ability to wait for more information as supplied by the NHC official forecast advisories which were discussed in Section II. This allows for the calibration of our multi-period evacuation decision model outcomes using actual forecast data.

Again, once a tropical depression, tropical storm, or hurricane has developed, the NHC issues an official forecast advisory every six hours at 5:00 a.m., 11:00 a.m., 5:00 p.m., and 11:00 p.m. Consequently, we can think of households potentially affected by the storm as being placed into a discrete time multi-period evacuation decision situation, where each discrete evacuation decision time period is six hours and is associated with a mutually exclusive NHC forecast advisory, denoted $\theta$. We assume that a storm's landfall at time $T$ is known with certainty ${ }^{50}$, and that the last safe possible time period for a household to evacuate, denoted by $T^{*}$, is six hours prior to $T$. As the 120 hour forecast is the maximum forecast time issued, let $n=0,1, \ldots, 19$ be the potential number of evacuation decision time periods from $T^{*}$ over the five-day forecast period such that we have $\left(T^{*}-19\right),\left(T^{*}-18\right), \ldots,\left(T^{*}-1\right), T^{*}$ potential evacuation decision time periods.

Let the $\left(T^{*}-n\right)$ current period forecast advisory, $\theta_{\left(T^{*}-n\right)}$, be a vector of $j$ possible states which describe a household's current status as it affects its evacuation decision (Dixit and Pindyck, 1994). At any $\left(T^{*}-n\right)$ current period the value of $\theta_{\left(T^{*}-n\right)}$ is known. However, as presented in Section II, hurricane forecasts contain a significant amount of uncertainty, with the degree of uncertainty decreasing as $\left(T^{*}-n\right)$ approaches $T$. Consequently, $\theta_{\left(T^{*}-n\right)}$ is a random variable which we assume follows a Markov process

\footnotetext{
${ }^{50}$ Regnier (2006) shows that landfall timing uncertainty ranges from 8.8 to 11.5 hours. We do not introduce this additional level of complexity into our model at this time.
} 
such that in the current period the probability that a particular realization of any of the possible $j$ current states occurs, $\theta_{\left(T^{*}-n\right)}^{j}$, depends only on the state in the previous period. For each pair of $(i, j)$ states, the probability of moving from state $i$ to $j$ is $p_{i j}$, with $\sum_{\mathrm{j}} p_{i j}=1$. For all states in each period, a Markov probability transition matrix is utilized to summarize all the information about the probability of $\theta_{\left(T^{*}-n\right)}$ moving across states from one period to the next (Adda, Cooper, 2003). Due to the inherent decreasing degree of uncertainty for $\theta_{\left(T^{*}-n\right)}$ as $\left(T^{*}-n\right)$ approaches $T$, our multi-period evacuation decision model uses Markov probability transition matrices that are nonstationary.

In each evacuation decision time period households face the binary choice of either to evacuate, or to wait one more time period for a revised hurricane forecast. If at any $\left(T^{*}-n\right)$ period the decision has been made to evacuate, this decision is not reversible as evacuation is assumed to be immediate and costs are sunk. For $n=1, \ldots, 19$, the household evacuation decision in each $\left(T^{*}-n\right)$ period is either to evacuate immediately given $\theta_{\left(T^{*}-n\right)}$, or to wait one period for more information from the expected updated forecast of $\left[E_{\left(T^{*}-n\right)}\left(\theta_{\left(T^{*}-n-1\right)}\right) \mid \theta_{\left(T^{*}-n\right)}\right]$ and the possibility of evacuating during period $\left(T^{*} *_{-}\right.$ $n-1)$. For $n=0$ the household evacuation decision in period $T^{*}$ is either to evacuate immediately given the now realized updated forecast of $\theta_{T^{*}}$, or to wait and simply ride out the storm at $T$.

Letting $V\left\{\theta_{\left(T^{*-n}\right)},\left(T^{*}-n\right)\right\}$ denote the value at time $\left(T^{*}-n\right)$ of having a forecast of $\theta_{\left(T^{*}-n\right)}$, each household faces the following optimal stopping problem: 


$$
V\left\{\theta_{\left(T^{*}-n\right)},\left(T^{*}-n\right)\right\}=\min \left\{c_{E V_{\left(T^{*}-n\right)}}, E_{\left(T^{*}-n\right)}\left[V\left\{\theta_{\left(T^{*}-n-1\right)},\left(T^{*}-n-1\right)\right\} \mid \theta_{\left(T^{*}-n\right)}\right]\right\}
$$

where $\quad E_{\left(T^{*}-n\right)}\left[V\left\{\theta_{\left(T^{*}-n-1\right)},\left(T^{*}-n-1\right)\right\} \mid \theta_{\left(T^{*}-n\right)}\right]=\sum_{\theta} V\left\{\theta_{\left(T^{*}-n-1\right)},\left(T^{*}-n-1\right)\right\} p\left(\theta_{\left(T^{*}-n-1\right)},\left(T^{*}-n\right)\right) \mid \theta_{\left(T^{*}-n\right)}$ and $p\left(\theta_{\left(T^{*-n-1}\right)},\left(T^{*}-n\right)\right) \mid \theta_{\left(T^{*}-n\right)}$ is the distribution of next period's landfall forecast given this period's landfall forecast. Given the short time horizon, there is no discounting.

Under specific cost of evacuating and waiting assumptions, we can show that a unique solution to (29) exists. Let us assume that $p\left(\theta_{\left(T^{*}-n-1\right)},\left(T^{*}-n\right)\right) \mid \theta_{\left(T^{*}-n\right)}$ is increasing in $\theta_{\left(T^{*}-n\right)}$ for all $\theta_{\left(T^{*}-n-1\right)}$. Thereby, $E_{\left(T^{*}-n\right)}\left[V\left\{\theta_{\left(T^{*}-n-1\right)},\left(T^{*}-n-1\right)\right\} \mid \theta_{\left(T^{*}-n\right)}\right]$ is increasing in $\theta_{\left(T^{*}-n\right)}$ for all $\theta_{\left(T^{*}-n-1\right)}$. If we further assume that $c_{E V_{\left(T^{*}-n\right)}}$ are constant across $\theta_{\left(T^{*}-n\right)}$ (as we did in our two-period model), then we can show that $V\left\{\theta_{\left(T^{*}-n\right)},\left(T^{*}-n\right)\right\}$ is increasing in $\theta_{\left(T^{*}-n\right)}$ and a unique solution to (29) exists, denoted $E V_{\left(T^{*}-n\right)}$, such that waiting for another forecast is optimal if $\theta_{\left(T^{*}-n\right)}<E V_{\left(T^{*}-n\right)}$, and evacuation is chosen whenever $\theta_{\left(T^{*-n}\right)} \geq E V_{\left(T^{*}-n\right)}$. This result is illustrated below in Figure $21^{51}$. Remember though that this uniqueness property of the solution to (29) depends on the assumed form of $c_{E V_{\left(T^{*}-n\right)}}$ and we do not present this as a general result. Nevertheless, the intuition is such that for certain forms of $c_{E V_{\left(T^{*}-n\right)}}$ a unique cutoff for households exists where waiting is optimal on one side of the forecast, and evacuating on the other.

\footnotetext{
${ }^{51}$ While we have illustrated our curves as being linear, a smooth function would be expected
} 
Figure IV.21. Unique Solution to Optimal Stopping Problem with Constant Costs of Evac

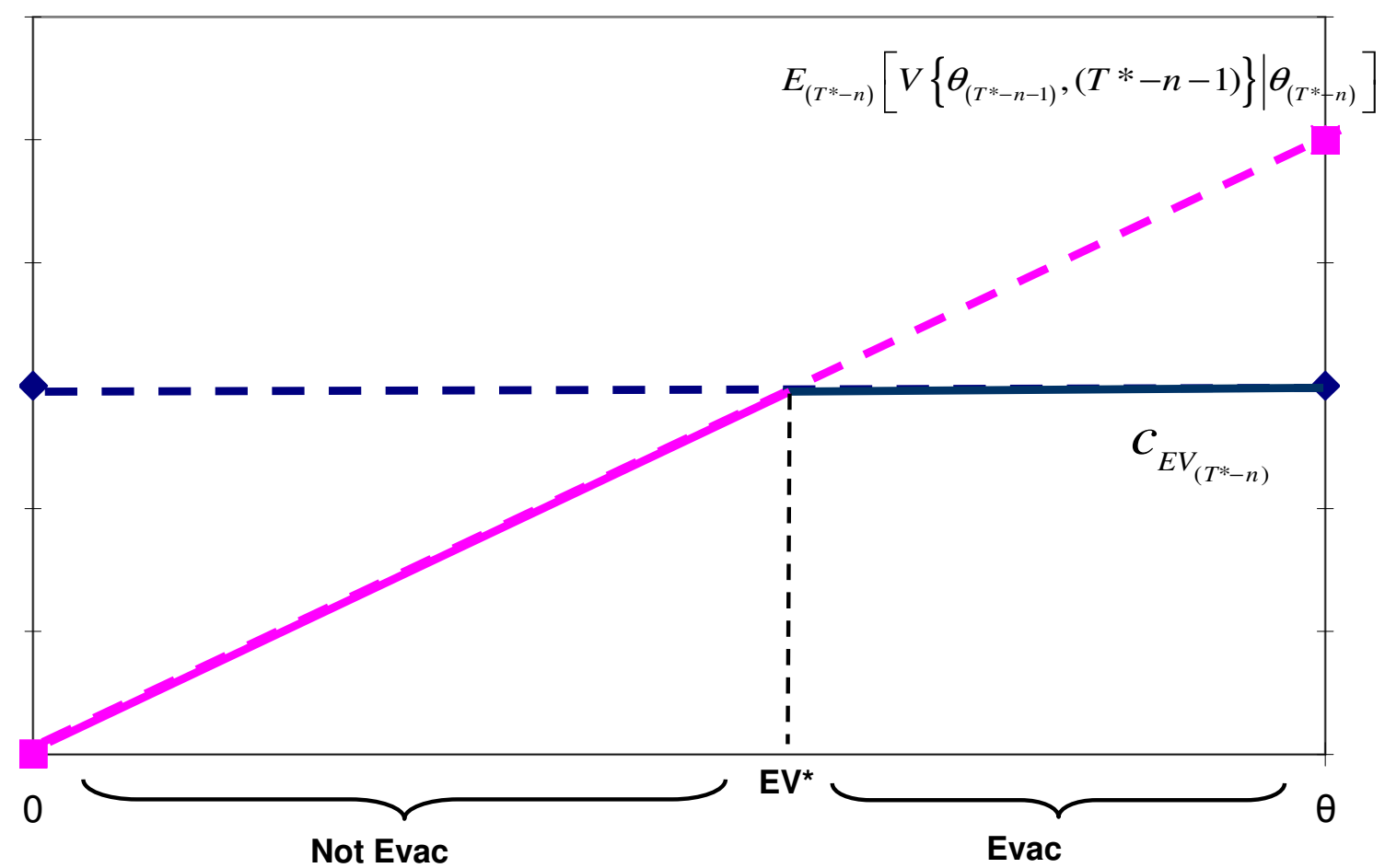

\section{IV.V. Model Inputs}

In order to solve our multi-period dynamic model of evacuation decision making, three main data inputs are needed: 1) for $n=0,1, \ldots, 19$, vector of $j$ possible forecast states, $\theta_{\left(T^{*}-n\right)}$, and their associated probability distributions, $\left.p\left(\theta_{\left(T^{*}-n-1\right)} \mid \theta_{\left(T^{*}-n\right)},\left(T^{*}-n\right)\right) ; 2\right)$ for $n=0,1, \ldots, 19$, the costs of evacuation, $c_{E V_{\left(T^{*}-n\right)}}$; and 3) for $T$, the expected costs of not evacuating, $c_{N_{-} E V_{(T)}}$. The construction of these inputs is detailed below. 


\section{State Space}

We construct our multi-period model state space (state variables and associated probability distributions) for a representative Gulf of Mexico location from historical storm forecast advisory and realized landfall data affecting 15 coastal locations in the Gulf of Mexico. Specifically, we use HURREVAC to stipulate a 900 nautical mile (NM) by $180 \mathrm{NM}$ Gulf of Mexico region that includes the 15 coastal locations listed in Table 2. We select 19 historical storm tracks from 1992-2005 passing through this region, and these are listed in Table 3 and illustrated in Figure $21^{52}$. The 15 coastal locations range between $28^{\circ}$ to $31^{\circ}$ North latitude and $82^{\circ}$ to $97^{\circ}$ West longitude, and are given explicit strike probabilities in HURREVAC as well as the NHC forecast archives. For the years 2004 and 2005 historical storm tracks for storms achieving either tropical storm or hurricane strength are identified, while for 1992-2003 only those storms making landfall as a hurricane are identified. Although we are only utilizing data from 19 storms, a healthy mixture of storm intensity levels and storm tracks are included.

Each forecast advisory contains a 12, 24, 36, 48, 72, 96, and 120 hour forecast of an approaching hurricane's center position (track forecast), maximum sustained surface wind speeds (intensity forecast), non-sustained wind speed gusts, and asymmetrical maximum wind radii from the storm's center. In order to construct the $\theta_{\left(T^{*}-n\right)}$ state space, we utilize the data related to the track and intensity forecasts, while ignoring the nonsustained wind speed and wind radii advisory information. The track forecast is provided by the NHC in degrees latitude and longitude, and the intensity forecast is given in knots,

\footnotetext{
${ }^{52}$ Figure 21 does not contain the storm track for Hurricane Charley, as Charley's eventual landfall was just south of our defined Gulf of Mexico region. However, most of Charley's forecast tracks did go through our defined region, and this is why the storm is included in the analysis.
} 
both of which are naturally continuous variables. However, specifying a discrete state space facilitates computational analysis of the model. Estimating exact solutions from a discrete state space must be weighed against a loss of information available from a continuous state space. Given that track and intensity forecasts provided in the NHC advisory are already discretized, with track forecasts given in specific degrees of latitude and longitude, and intensity forecasts rounded to 5 knots, this loss of information is minimal.

We discretize the intensity forecast through the use of the SSHS as presented previously in Table 1 of Section II. Not only does the SSHS naturally lend itself to this discretization, but it is reasonable in terms of the hurricane's intensity to assume that households focus on the forecasted SSHS category level of the hurricane as opposed to the storm's specific mph. We discretize the track forecast through the use of the strike probabilities. As discussed in Section II, the strike probabilities are issued in conjunction with the forecast advisories and are the percent chance that the center of the storm will cross within 75 miles of a location. In this way, the strike probabilities are able to provide a discrete way to handle the latitude and longitude coordinates of the forecast track for any particular location.

Through our discretization of the track and intensity forecasts we have two separate state variables: an intensity forecast state variable with 5 levels (the 5 category levels of SSHS); and a track forecast state variable with 100 levels. Given that the size of the state space is formed as the product of the number of levels of all the identified state variables (Kristensen, 1996), we have a state space $=500$ states. That is, we have to 
Table IV.2. 15 Coastal Gulf of Mexico Locations - County/Parish (Nearest Major City)

\begin{tabular}{|c|c|c|}
\hline \# & $\underline{\text { State }}$ & $\underline{\text { Locations }}$ \\
\hline$\overline{1}$ & $\overline{\mathrm{TX}}$ & $\overline{\text { Calhoun County (Port Lavaca / Port O Connor) }}$ \\
\hline 2 & & Brazoria County (Freeport) \\
\hline 3 & & Galveston County (Galveston) \\
\hline 4 & & Jefferson County (Port Arthur) \\
\hline 5 & LA & Iberia Parish (New Iberia) \\
\hline 6 & & St. Charles Parish (New Orleans) \\
\hline 7 & & Plaquemines Parish (Buras) \\
\hline 8 & MS & Harrison County (Gulfport) \\
\hline 9 & $\mathrm{AL}$ & Mobile County (Mobile) \\
\hline 10 & FL & Escambia County (Pensacola) \\
\hline 11 & & Bay County (Panama City) \\
\hline 12 & & Franklin County (Apalachicola) \\
\hline ( & & Wakulla County (St. Marks) \\
\hline 1 & & Levy County (Cedar Key) \\
\hline & & Hillsborough County (Tampa) \\
\hline
\end{tabular}

Table IV.3. 19 Identified Gulf of Mexico Storms

\begin{tabular}{|c|c|c|c|c|}
\hline \# & Year & Storm & $\frac{\text { Landfall }}{\text { CAT }}$ & $\frac{\text { Max }}{\text { CAT }}$ \\
\hline$\overline{1}$ & $\overline{2005}$ & $\overline{\text { Arlene }}$ & 0 & 0 \\
\hline 2 & & Cindy & 0 & 0 \\
\hline 3 & & Dennis & 3 & 4 \\
\hline 4 & & Katrina & 4 & 5 \\
\hline 5 & & Rita & 3 & 5 \\
\hline 6 & 2004 & Bonnie & 0 & 0 \\
\hline 7 & & Charley & 4 & 4 \\
\hline 8 & & Frances & 0 & 4 \\
\hline 9 & & Ivan & 3 & 5 \\
\hline 10 & & Matthew & 0 & 0 \\
\hline 11 & 2003 & Claudette & 1 & 1 \\
\hline 12 & 2002 & Lili & 1 & 4 \\
\hline 13 & 1998 & Earl & 1 & 2 \\
\hline 14 & & Georges & 2 & 4 \\
\hline 15 & 1997 & Danny & 1 & 1 \\
\hline 16 & 1995 & Allison & 1 & 1 \\
\hline 17 & & Erin & 1 & 1 \\
\hline 18 & & Opal & 3 & 4 \\
\hline 19 & 1992 & Andrew & 3 & 5 \\
\hline
\end{tabular}


Figure IV.22. 19 Identified Gulf of Mexico Historical Storm Tracks

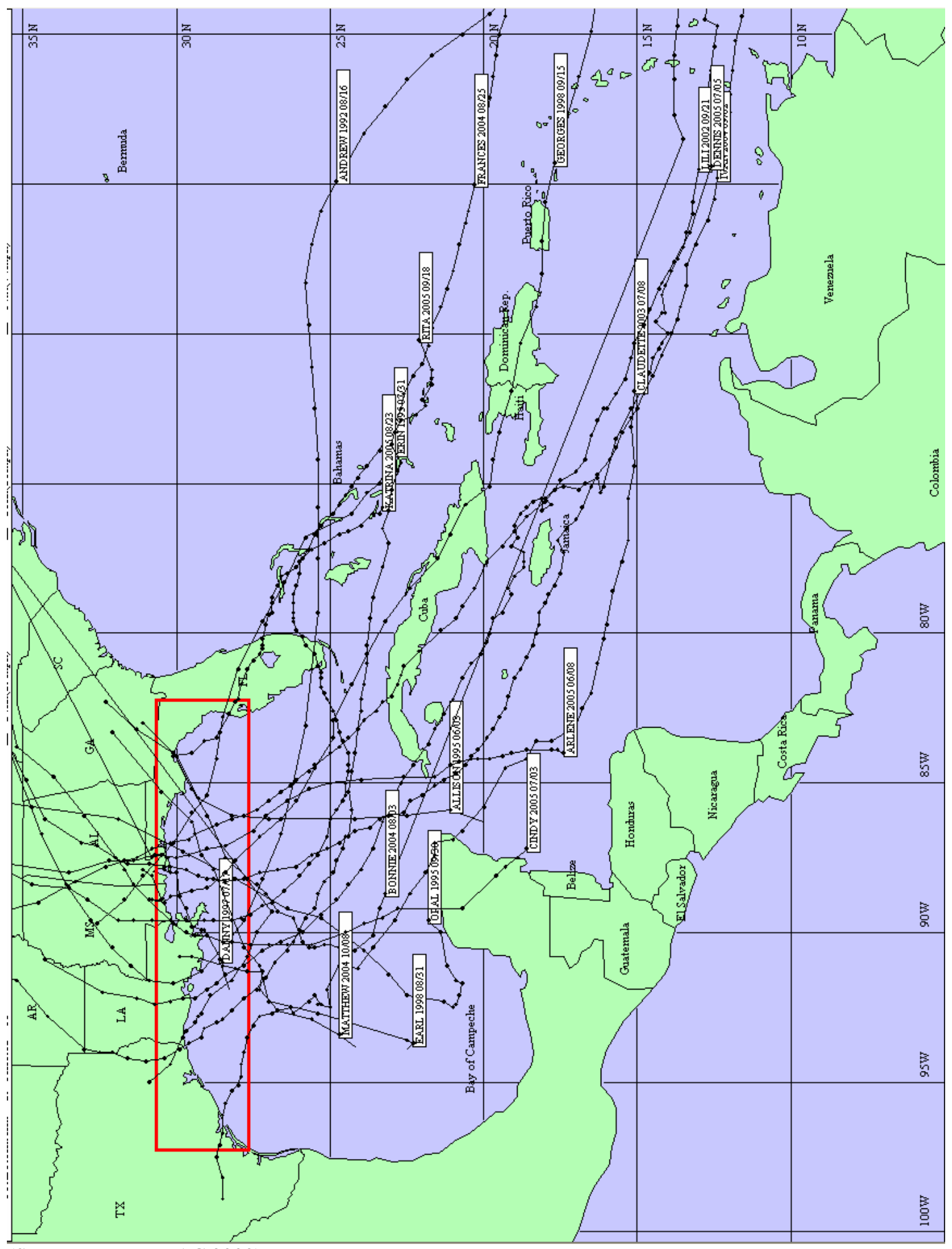

(Source: HURREVAC 2000)

(Note: Hurricane Charley is not included in this graphic) 
solve (29) at 500 points in each evacuation time period, which creates a significant curse of dimensionality. While a 500 point state space is perhaps not that large, dimensionality issues arise in the construction of our 500 point state space's associated Markov probability transition matrix, which has dimensions of $500 \times 500$. Given that we are working with a limited amount of data from 19 storms, and also that our transition matrices are nonstationary, the $500 \times 500$ dimensionality of the transition matrix creates significant difficulties.

In order to alleviate the dimensionality issues of the transition matrices, we construct a single discretized state variable which we call a hurricane forecast "risk index". The index combines the intensity and track forecast information into a scalar. Furthermore, this discretized risk index state variable is similar to the 2006 wind speed probability products issued by the NHC in that it provides a single and less complicated source of information on the probability of winds of a certain strength affecting a given location. The hurricane forecast risk indices are constructed for each of the 15 coastal locations along the Gulf of Mexico for each $\left(T^{*}-n\right)$ period with $n=0,1, \ldots, 19$.

At landfall, our discretized risk index state variable is straightforward to illustrate as $\theta$ is no longer a forecast, but rather a realized value for any number of identified locations. Thus, at landfall our discretization of $\theta_{T}$ not only includes states 1 through 5 corresponding to the five SSHS CAT levels, but also a state 0 that corresponds to storms that either fall below the CAT 1 hurricane designation, or actual CAT 1-5 hurricanes that do not make landfall at a particular location. For example, in 2005 Hurricane Dennis made landfall in Pensacola, FL as a CAT 3 hurricane. Here, $\theta_{T}=3$ for Pensacola, but $\theta_{T}$ $=0$ for all other locations along the Gulf Coast that at some point had the possibility of 
being struck by Hurricane Dennis, such as Port Arthur, TX. Table 4 presents the 6 discretized state variable intervals we use in our model for period $T$ only.

Table IV.4. Discretized State Variable, $\theta_{T}$

\begin{tabular}{|c|c|c|}
\hline State $\theta_{T}$ & $\begin{array}{c}\text { Hurricane } \\
\text { Category Level }\end{array}$ & $\begin{array}{c}\text { Wind Speed } \\
(\mathrm{mph})\end{array}$ \\
\hline 0 & 0 & $<74$ \\
\hline 1 & 1 & $74-95$ \\
\hline 2 & 2 & $96-110$ \\
\hline 3 & 3 & $111-130$ \\
\hline 4 & 4 & $131-155$ \\
\hline 5 & 5 & $>155$ \\
\hline
\end{tabular}

While similar to the $\theta_{T}$ discretization, the discretization of $\theta_{\left(T^{*}-n\right)}$ for $n=0,1, \ldots$, 19 is more complex. The detailed steps involved in the construction of the discretized risk index state variables, $\theta_{\left(T^{*}-n\right)}$ for $n=0,1, \ldots, 19$, and their associated probability transition matrices is as follows:

\section{Steps 1) to 5) - Incorporating Storm and Track Uncertainty}

1) For each 5:00 AM, 11:00 AM, 5:00 PM, and 11:00 PM NHC forecast advisory issued per day, through the life of each storm, the associated $12,24,36,48,72,96$, and 120 hour intensity forecast of specific windspeed (mph) is collected from HURREVAC ${ }^{53}$

2) The 12, 24, 36, 48, 72, 96, and 120 hour average intensity errors for 1996-2005 (NHC, 2006) are added and subtracted to the specific 12, 24, 36, 48, 72, 96, and 120 forecasted windspeeds collected from step 1) to determine an average range of probable wind speed values.

\footnotetext{
${ }^{53}$ HURREVAC sources its forecast data from the NHC and transforms it to mph from kt. As mentioned previously, prior to 2003 forecasts beyond 72 hours were not provided in the NHC forecast advisory. Also, for the years 2003-2005 not every forecast advisory contains forecasts up to 120 hours.
} 
The average intensity errors for 1996-2005 are presented in Table 5.

Table IV.5. Average NHC 10-Year Intensity Forecast Errors (1996-2005)

\begin{tabular}{|c|c|c|}
\hline Forecast Hour & $\underline{\text { Knots }}$ & MPH \\
\hline 0 & 2.2 & 2.5 \\
\hline $6 *$ & 4.3 & 4.9 \\
\hline 12 & 6.4 & 7.4 \\
\hline 24 & 10.1 & 11.6 \\
\hline 36 & 12.7 & 14.6 \\
\hline 48 & 14.9 & 17.1 \\
\hline 72 & 18.7 & 21.5 \\
\hline 96 & 19.8 & 22.8 \\
\hline 120 & 21.8 & 25.1 \\
\hline
\end{tabular}

* The 6 hour forecast is not from the NHC, but rather is an average of the 0 and 12 hour forecast errors

3) From the average range of probable windspeed values generated in step 2), the percentage of the range that falls into the SSHS category $0,1,2,3,4$, and 5 is calculated.

An example of steps 1 to 3 for the 7/9/2005 5:00 AM Hurricane Dennis advisory (advisory \#19) is given in Table 6. Note, that while the 0 hour forecast indicated in Table 6 is 5:00 AM, the actual data collected for this advisory occurs three hours prior to the advisory at 2:00 AM.

4) Strike probabilities are collected from HURREVAC ${ }^{54}$ for the 15 coastal locations along the Gulf of Mexico.

An example of the provided strike probabilities from HURREVAC for the 7/9/2005 5:00 AM Hurricane Dennis advisory (advisory \#19) was previously given in Figure 5 of Section II.

\footnotetext{
${ }^{54}$ When HURREVAC strike probability information was missing, the strike probabilities were collected directly from the NHC Hurricane Season Tropical Cyclone Product Archives at http://www.nhc.noaa.gov/pastall.shtml, which is the source of the HURREVAC data
} 
Table IV.6. Construction of Risk Indices, Steps 1) to 3)

\begin{tabular}{|c|c|c|c|c|c|c|c|c|c|c|c|}
\hline $\begin{array}{c}\text { Forecast } \\
\text { (hr) }\end{array}$ & Date & $\begin{array}{l}\text { Wind } \\
(\mathbf{m p h})\end{array}$ & $\begin{array}{l}\text { Wind } \\
\text { min }\end{array}$ & $\begin{array}{l}\text { Wind } \\
\max \end{array}$ & $\begin{array}{l}\text { Wind } \\
\text { range }\end{array}$ & $\mathbf{0}$ & 1 & 2 & 3 & 4 & 5 \\
\hline 0 & 7/09 $5 \mathrm{AM}$ & 92 & & & & & & & & & \\
\hline 12 & 7/09 2 PM & 103 & 95.6 & 110.4 & 15.7 & $0 \%$ & $2 \%$ & $98 \%$ & $0 \%$ & $0 \%$ & $0 \%$ \\
\hline 24 & 7/10 $2 \mathrm{AM}$ & 115 & 103.4 & 126.6 & 24.2 & $0 \%$ & $0 \%$ & $31 \%$ & $69 \%$ & $0 \%$ & $0 \%$ \\
\hline 36 & 7/10 2 PM & 126 & 111.4 & 140.6 & 30.2 & $0 \%$ & $0 \%$ & $0 \%$ & $65 \%$ & $35 \%$ & $0 \%$ \\
\hline 48 & 7/112 AM & 52 & 34.9 & 69.1 & 35.3 & $100 \%$ & $0 \%$ & $0 \%$ & $0 \%$ & $0 \%$ & $0 \%$ \\
\hline 72 & 7/12 $2 \mathrm{AM}$ & 34 & 12.5 & 55.5 & 44.0 & $100 \%$ & $0 \%$ & $0 \%$ & $0 \%$ & $0 \%$ & $0 \%$ \\
\hline 96 & 7/13 $2 \mathrm{AM}$ & 23 & 0.2 & 45.8 & 46.6 & $100 \%$ & $0 \%$ & $0 \%$ & $0 \%$ & $0 \%$ & $0 \%$ \\
\hline 120 & 7/14 2 AM & 23 & -2.1 & 48.1 & 51.2 & $100 \%$ & $0 \%$ & $0 \%$ & $0 \%$ & $0 \%$ & $0 \%$ \\
\hline
\end{tabular}

5) The strike probabilities for each location are then multiplied by the derived intensity error range from step 3) to obtain by location, a probability of a strike by CAT level per forecast hour, where CAT 0 indicates either a tropical storm direct hit on that location, or the center of the storm missing the location.

An example of this for Pensacola, FL based on advisory \#19 from Hurricane Dennis is given in Figure 23. Following our methodology, Figure 23 indicates that 36 hours from the 7/9/2005 5:00 AM advisory there is a 68\% chance that Hurricane Dennis will miss Pensacola (the center of the storm will not come within 75 miles), but if it strikes Pensacola, there is a $21 \%$ chance it will strike as a CAT 3 hurricane, or an $11 \%$ chance it will strike as a CAT 4. 
Figure IV.23. Example of the Determination of a Probability Of A Strike By CAT Level Per Forecast Hour

\begin{tabular}{|c|c|c|c|c|c|c|c|c|}
\hline Location & $\frac{\text { Strike }}{\text { Probability }}$ & & & & & & & \\
\hline$\overline{\text { Tampa }}$ & 0.17 & & & & & & & \\
\hline Cedar Key & 0.18 & & & & & & & \\
\hline St. Marks & 0.24 & & & & & & & \\
\hline Apalachicola & 0.33 & & & & & & & \\
\hline Panama City & 0.34 & Forecast hr & $\underline{\mathbf{0}}$ & $\underline{1}$ & $\underline{2}$ & $\underline{\mathbf{3}}$ & $\underline{4}$ & $\underline{5}$ \\
\hline Pensacola & 0.32 & 12 & $68 \%$ & $1 \%$ & $31 \%$ & $0 \%$ & $0 \%$ & $0 \%$ \\
\hline Mobile & 0.28 & 24 & $68 \%$ & $0 \%$ & $10 \%$ & $22 \%$ & $0 \%$ & $0 \%$ \\
\hline Gulfport & 0.24 & 36 & $68 \%$ & $0 \%$ & $0 \%$ & $21 \%$ & $11 \%$ & $0 \%$ \\
\hline Buras & 0.17 & 48 & $100 \%$ & $0 \%$ & $0 \%$ & $0 \%$ & $0 \%$ & $0 \%$ \\
\hline New Orleans & 0.14 & 72 & $100 \%$ & $0 \%$ & $0 \%$ & $0 \%$ & $0 \%$ & $0 \%$ \\
\hline New Iberia & 0.05 & 96 & $100 \%$ & $0 \%$ & $0 \%$ & $0 \%$ & $0 \%$ & $0 \%$ \\
\hline Port Arthur & 0 & 120 & $100 \%$ & $0 \%$ & $0 \%$ & $0 \%$ & $0 \%$ & $0 \%$ \\
\hline Galveston & 0 & & & & & & & \\
\hline Freeport & 0 & & & & & & & \\
\hline Port Lavaca & 0 & & & & & & & \\
\hline
\end{tabular}

So while steps 1) to 3) discretize the intensity forecast, step 4) discretizes the track forecast by location and step 5) combines the discretized intensity and track forecasts.

Steps 6) to 8) - Creating per period, per storm risk indices by location

6) Landfall, $T$, and the locations directly hit by the center of a storm are determined by stepping through the actual track of the storm in HURREVAC ${ }^{55}$. The last safe possible time period to evacuate, $T^{*}$, is the last official advisory preceding the determined $T$. In nearly all cases, this is six hours preceding $T$.

Figure 24 indicates the HURREVAC determined landfall at Pensacola, FL and Mobile, AL for Hurricane Dennis as shown by the $99 \%$ strike probabilities.

\footnotetext{
55 As this is accomplished through a visual inspection, this is ultimately a subjective determination. However, landfall by location was verified through other storm summaries/post-storm assessments where available.
} 
Figure IV.24. Hurricane Dennis Landfall Locations

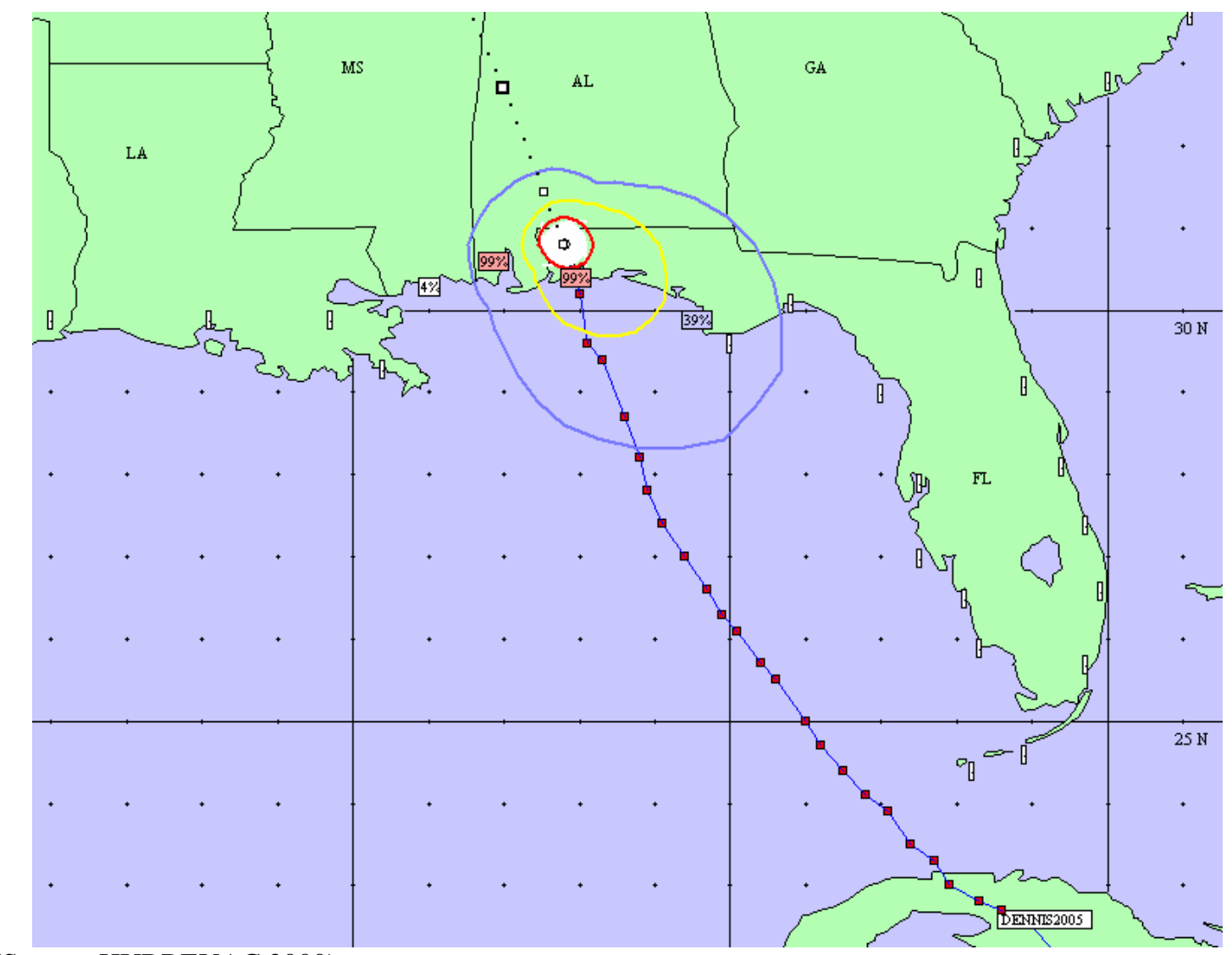

(Source: HURREVAC 2000)

7) Landfall forecasts for each of the other $\left(T^{*}-n\right)$ periods, $n=1,2,3, \ldots, 19$ are determined through a combination of visual inspection of landfall points from HURREVAC, and use of the NHC forecast advisory archives where the forecast of landfall is indicated as the storm being "inland". Note, this is not a determined landfall forecast for a particular location, but rather a generic indication of landfall somewhere within the Gulf region, as the specific landfall point is constantly changing within the region based upon the updated forecasts. 
Table 7 provides the step 7) determined generic Gulf Region landfall forecasts for Hurricane Dennis for periods $T^{*}$ to $\left(T^{*}-11\right)$. Actual landfall occurred on $7 / 10 / 05$ at approximately 3:00 PM. $T^{*}$ is $7 / 10 / 05$ at 11:00 AM, the last official NHC advisory preceding landfall. Since the earliest available forecast at $T^{*}$ is 12 hours, which would be well past the point of landfall, we assume that households view $T^{*}$ as what to expect at landfall with an average intensity error being the average of the $0 \mathrm{hr}$ and $12 \mathrm{hr}$ forecasts. We see from Table 7 that all of the $\left(T^{*}-1\right)$ to $\left(T^{*}-11\right)$ forecasts indicate landfall on $7 / 10$, with an average forecasted windspeed of $126 \mathrm{mph}$, which closely mirrors the actual landfall values.

Table IV.7. Hurricane Dennis $\left(T^{*}-n\right)$ Forecasted Landfall Points

\begin{tabular}{|c|c|c|c|}
\hline $\begin{array}{c}\left(\mathrm{T}^{*} \text {-n }\right) \\
\text { Advisory }\end{array}$ & Forecast $(\mathbf{h r})$ & Landfall Date & MPHWind \\
\hline$(\mathrm{T} *-11)$ & 72 & $7 / 10 / 20052 \mathrm{PM}$ & 121 \\
\hline$\left(\mathrm{T}^{*}-10\right)$ & 72 & $7 / 10 / 20058 \mathrm{PM}$ & 115 \\
\hline$\left(\mathrm{T}^{*}-9\right)$ & 48 & $7 / 10 / 20052 \mathrm{AM}$ & 132 \\
\hline$\left(\mathrm{T}^{*}-8\right)$ & 48 & $7 / 10 / 20058 \mathrm{AM}$ & 132 \\
\hline$\left(\mathrm{T}^{*}-7\right)$ & 48 & $7 / 10 / 20052 \mathrm{PM}$ & 126 \\
\hline$\left(\mathrm{T}^{*}-6\right)$ & 48 & $7 / 10 / 20058 \mathrm{PM}$ & 115 \\
\hline$\left(\mathrm{T}^{*}-5\right)$ & 36 & $7 / 10 / 20052 \mathrm{PM}$ & 126 \\
\hline$\left(\mathrm{T}^{*}-4\right)$ & 24 & $7 / 10 / 20058 \mathrm{AM}$ & 109 \\
\hline$\left(\mathrm{T}^{*}-3\right)$ & 24 & $7 / 10 / 20052 \mathrm{PM}$ & 126 \\
\hline$\left(\mathrm{T}^{*}-2\right)$ & 12 & $7 / 10 / 20058 \mathrm{AM}$ & 138 \\
\hline$\left(\mathrm{T}^{*}-1\right)$ & 12 & $7 / 10 / 20052 \mathrm{PM}$ & 144 \\
\hline $\mathrm{T}^{*}$ & & $7 / 10 / 200511 \mathrm{AM}$ & 138 \\
\hline Landfall $(\mathrm{T})$ & & $7 / 10 / 20053 \mathrm{PM}$ & 120 \\
\hline
\end{tabular}

However, Table 7 also illustrates a limitation in the analysis regarding the assumption that landfall is known with certainty. While $\left(T^{*}-9\right)$ is 3 days out from the landfall, the landfall forecast period selected for $\left(T^{*}-9\right)$ is a 48 hour forecast. Ostensibly, including all of the 12, 24, 36, etc. hour forecasts as an additional state variable in regard 
to the timing of landfall could be an addition to the model, instead of selecting a known landfall forecast. This would add an additional level of dimensionality complexity to our state space and we do not include it at this time.

8) Risk indices per storm, per location, and per period are calculated by multiplying the probability of a strike by CAT level from step 5) for the selected landfall forecast periods from step 7) by the $0,1,2,3,4$, and 5 SSHS CAT levels.

Table 8 gives an example of the $\left(T^{*}-n\right)$ period, $\mathrm{n}=0,1, \ldots, 11$, generated risk indices for Pensacola, FL for Hurricane Dennis. For example, the $\left(T^{*}-11\right)$ risk index is estimated by $(0 \times .82)+(1 \times .0)+(2 \times .05)+(3 \times .08)+(4 \times .05)+(5 \times .0)=0.54$. Table 8 illustrates that while our generated risk indices are based upon the SSHS, the uncertainty in the intensity and track information does not allow for a direct comparison. For example, while the $\left(T^{*} 7\right)$ forecast is predicting a CAT 3 hurricane (126 mph) making landfall somewhere in the Gulf region, the constructed risk index by location which incorporates the uncertainty of the track and the average intensity errors for a 48 hour forecast, equates to a 0.73 value for Pensacola, FL at the $\left(T^{*}-7\right)$ evacuation decision period. For Pensacola, the risk index evolves from 0.54 at $\left(T^{*}-11\right)$ to 3.76 at $\left(T^{*}\right)$, with landfall being 3.00 at $T$. A complete listing of the estimated risk indices per storm, per location, and per period is provided in a separate appendix. 
Table IV.8. Pensacola, FL Risk Indices for Hurricane Dennis

\begin{tabular}{|c|c|c|c|c|c|c|c|c|c|c|}
\hline $\begin{array}{c}\left(\mathbf{T}^{*}-\mathbf{n}\right) \\
\text { Advisory }\end{array}$ & $\begin{array}{c}\text { Forecast } \\
\text { (hr) }\end{array}$ & $\begin{array}{l}\text { Landfall } \\
\text { Date }\end{array}$ & $\begin{array}{l}\text { Wind } \\
(\mathbf{m p h})\end{array}$ & $\underline{\mathbf{0}}$ & $\underline{1}$ & $\underline{2}$ & $\underline{\mathbf{3}}$ & $\underline{4}$ & $\underline{5}$ & Risk \\
\hline$\left(\mathrm{T}^{*}-11\right)$ & 72 & 7/10 2 PM & 121 & $82 \%$ & $0 \%$ & $5 \%$ & $8 \%$ & $5 \%$ & $0 \%$ & 0.54 \\
\hline$\left(\mathrm{T}^{*}-10\right)$ & 72 & 7/10 $8 \mathrm{PM}$ & 115 & $81 \%$ & $1 \%$ & $6 \%$ & $9 \%$ & $3 \%$ & $0 \%$ & 0.51 \\
\hline$\left(\mathrm{T}^{*}-9\right)$ & 48 & 7/10 2 AM & 132 & $82 \%$ & $0 \%$ & $0 \%$ & $8 \%$ & $10 \%$ & $0 \%$ & 0.64 \\
\hline$\left(\mathrm{T}^{*}-8\right)$ & 48 & 7/10 8 AM & 132 & $80 \%$ & $0 \%$ & $0 \%$ & $9 \%$ & $11 \%$ & $0 \%$ & 0.71 \\
\hline$\left(\mathrm{T}^{*}-7\right)$ & 48 & 7/10 2 PM & 126 & $78 \%$ & $0 \%$ & $1 \%$ & $12 \%$ & $8 \%$ & $0 \%$ & 0.73 \\
\hline$\left(T^{*}-6\right)$ & 48 & 7/10 8 PM & 115 & $75 \%$ & $0 \%$ & $9 \%$ & $14 \%$ & $2 \%$ & $0 \%$ & 0.67 \\
\hline$\left(\mathrm{T}^{*}-5\right)$ & 36 & 7/10 2 PM & 126 & $68 \%$ & $0 \%$ & $0 \%$ & $21 \%$ & $11 \%$ & $0 \%$ & 1.07 \\
\hline$\left(\mathrm{T}^{*}-4\right)$ & 24 & 7/10 8 AM & 109 & $64 \%$ & $0 \%$ & $20 \%$ & $16 \%$ & $0 \%$ & $0 \%$ & 0.88 \\
\hline$\left(T^{*}-3\right)$ & 24 & 7/10 2 PM & 126 & $65 \%$ & $0 \%$ & $0 \%$ & $24 \%$ & $11 \%$ & $0 \%$ & 1.16 \\
\hline$\left(T^{*}-2\right)$ & 12 & 7/10 8 AM & 138 & $62 \%$ & $0 \%$ & $0 \%$ & $1 \%$ & $37 \%$ & $0 \%$ & 1.51 \\
\hline$\left(\mathrm{T}^{*}-1\right)$ & 12 & 7/10 2 PM & 144 & $50 \%$ & $0 \%$ & $0 \%$ & $0 \%$ & $50 \%$ & $0 \%$ & 2.00 \\
\hline $\mathrm{T}^{*}$ & & 7/10 11 AM & I 138 & $6 \%$ & $0 \%$ & $0 \%$ & $0 \%$ & $94 \%$ & $0 \%$ & 3.76 \\
\hline Landfall (T) & & 7/10 3 PM & 120 & & & & & & & \\
\hline
\end{tabular}

These constructed hurricane forecast risk indices represent the discretized state variable, $\theta_{\left(T^{*}-n\right)}$ for $n=0,1, \ldots, 19$, and capture the intensity and track forecast information in a single state variable that avoids dimensionality issues while allowing for a smooth transition into the landfall discretized state variables of $\theta_{T}$. However, the way the risk indices have been constructed introduces an additional loss of information. For example, the $\left(T^{*}-1\right)$ risk index in Table 8 above is $($ CAT $0 \times 50 \%)+($ CAT $4 \times 50 \%)=$ 2.00. One can imagine a multitude of other values that also equate to a 2.00 risk index, but that connotate a different level of risk to households such as CAT $1=50 \%$ and CAT 
$3=50 \%$, or CAT $1=25 \%$, CAT $2=50 \%$, and CAT $3=25 \%$, etc. We accept this risk information limitation to our constructed risk indices.

Steps 9) to 11) - Creating the Total Transition Matrices from Individual Location Risk $\underline{\text { Indices }}$

9) For all 15 locations the risk indices are aggregated by year. The total number of observations per year (based on the number of storms selected for that year) are assigned to the appropriate probability transition matrix categorization for each period.

For example, in 2002 only Hurricane Lili was selected for our analysis. Table 9 presents the final risk indices for all locations in 2002.

Table IV .9. 2002 Risk Indices for All Locations

\begin{tabular}{|c|c|c|c|c|c|c|c|c|c|c|c|c|c|}
\hline Locations & $\underline{\mathbf{T}}$ & $\underline{\mathbf{T}^{*}}$ & $(\mathrm{~T} *-1)$ & $\left(T^{*}-2\right)$ & $(\mathbf{T} *-3)$ & $\left(T^{*}-4\right)$ & $\left(T^{*}-5\right)$ & $(T *-6)$ & $(\mathrm{T} *-7)$ & $(T *-8)$ & $\left(T^{*}-9\right)$ & $\left(T^{*}-10\right)$ & $\left(T^{*}-11\right)$ \\
\hline Tampa & 0.00 & 0.00 & 0.00 & 0.00 & 0.00 & 0.00 & 0.00 & 0.00 & 0.00 & 0.00 & 0.06 & 0.06 & 0.15 \\
\hline Cedar Key & 0.00 & 0.00 & 0.00 & 0.00 & 0.00 & 0.00 & 0.00 & 0.00 & 0.00 & 0.00 & 0.06 & 0.06 & 0.12 \\
\hline St. Marks & 0.00 & 0.00 & 0.00 & 0.00 & 0.07 & 0.09 & 0.06 & 0.06 & 0.06 & 0.09 & 0.06 & 0.06 & 0.12 \\
\hline Apalachicola & 0.00 & 0.00 & 0.00 & 0.00 & 0.10 & 0.09 & 0.09 & 0.06 & 0.09 & 0.15 & 0.12 & 0.12 & 0.18 \\
\hline Panama City & 0.00 & 0.00 & 0.00 & 0.00 & 0.13 & 0.15 & 0.12 & 0.12 & 0.12 & 0.18 & 0.15 & 0.12 & 0.21 \\
\hline Pensacola & 0.00 & 0.00 & 0.08 & 0.24 & 0.33 & 0.33 & 0.27 & 0.24 & 0.24 & 0.30 & 0.24 & 0.18 & 0.24 \\
\hline Mobile & 0.00 & 0.09 & 0.44 & 0.56 & 0.50 & 0.42 & 0.36 & 0.33 & 0.30 & 0.36 & 0.27 & 0.21 & 0.24 \\
\hline Gulfport & 0.00 & 0.15 & 0.88 & 0.76 & 0.60 & 0.49 & 0.42 & 0.39 & 0.39 & 0.42 & 0.36 & 0.27 & 0.30 \\
\hline Buras & 0.00 & 0.09 & 1.12 & 0.80 & 0.66 & 0.55 & 0.52 & 0.51 & 0.48 & 0.48 & 0.45 & 0.36 & 0.39 \\
\hline New Orleans & 0.00 & 0.93 & 1.88 & 1.00 & 0.76 & 0.64 & 0.58 & 0.55 & 0.48 & 0.48 & 0.42 & 0.33 & 0.36 \\
\hline New Iberia & 1.00 & 2.49 & 2.72 & 1.48 & 0.96 & 0.79 & 0.70 & 0.64 & 0.55 & 0.51 & 0.45 & 0.36 & 0.39 \\
\hline ort Arthur & 0.00 & 0.63 & 0.64 & 1.24 & 0.89 & 0.76 & 0.70 & 0.64 & 0.55 & 0.48 & 0.45 & 0.36 & 0.36 \\
\hline Galveston & 0.00 & 0.00 & 0.00 & 0.76 & 0.73 & 0.64 & 0.64 & 0.61 & 0.55 & 0.45 & 0.42 & 0.36 & 0.36 \\
\hline Freeport & 0.00 & 0.00 & 0.00 & 0.36 & 0.56 & 0.52 & 0.55 & 0.55 & 0.51 & 0.42 & 0.42 & 0.36 & 0.33 \\
\hline ort Lavaca & 0.00 & 0.00 & 0.00 & 0.00 & 0.27 & 0.30 & 0.39 & 0.39 & 0.42 & 0.33 & 0.36 & 0.30 & 0.27 \\
\hline
\end{tabular}


Hurricane Lili landfall occurred at the New Iberia location as a CAT 1 hurricane. Based upon the Table 9 data, Table 10 shows the aggregated probability transition matrix for $T^{*}$ to $T$ in 2002 . As there is only one storm for 2002, each aggregated probability transition matrix per period, including $T^{*}$ to $T$, is based upon 15 observations. In contrast, the yearly matrices for 2005 and 2004 are based upon 75 observations each, as data from five storms was collected for both of these years.

Table IV.10. $2002 T^{*}$ to $T$ Aggregate Transition Matrix

\begin{tabular}{|c|c|c|c|c|c|c|}
\hline $\mathbf{T}^{*}$ & $\frac{T}{0}$ & 1 & 2 & 3 & 4 & 5 \\
\hline 0 & 9 & & & & & \\
\hline $0-0.5$ & 3 & & & & & \\
\hline $0.5-1$ & 2 & & & & & \\
\hline $1-1.5$ & & & & & & \\
\hline $1.5-2$ & & & & & & \\
\hline $2-2.5$ & & 1 & & & & \\
\hline $2.5-3$ & & & & & & \\
\hline $3-3.5$ & & & & & & \\
\hline $3.5-4$ & & & & & & \\
\hline $4-4.5$ & & & & & & \\
\hline $4.5-5$ & & & & & & \\
\hline
\end{tabular}

Notice from Table 10 that the number of state levels vary by time period such that for $T$ we have defined levels $0,1, \ldots, 5$ which correspond directly to the SSHS, while for $T^{*}$ we have defined state levels $0,[0-0.5],[0.5-1],[1-1.5], \ldots,[4.5-5]$ which correspond to the SSHS in a slightly altered way. However, these states do change over time to reflect the inherent constraints of the strike probabilities, i.e., for the $12,24,36,48$, and 72 hour forecasts, the maximum strike probability issued by the NHC is $60-80 \%, 35$ $50 \%, 20-25 \%, 13-18 \%$, and $10 \%$ respectively. Therefore, the further the evacuation 
decision period is from $T^{*}$, the lower will be the observed risk indices such that states 0 , $[0-0.25],[0.25-0.5],[0.5-0.75],[0.75-1],[1-1.5],[1.5-2],[2-3],[3-4]$, and [4-5] are defined for periods $\left(T^{*}-2\right)$ to $\left(T^{*}-6\right)$, and states $0,[0-0.25],[0.25-0.5],[0.5-0.75],[0.75-$ 1], [1-2], [2-3], [3-4], and [4-5] are defined for periods $\left(T^{*}-7\right)$ to $\left(T^{*}-11\right)$.

10) The yearly probability matrices from 1992 - 2005 are aggregated into the final probability transition matrices per each period.

Table 11 provides an example of the Markov transition matrix for $\theta$ moving from $T^{*}$ to $T$ that is used in our multi-period evacuation decision model. From Table 11 we see that the probability of moving from our discretized $T^{*}$ state of [2-2.5] to state 3 at $T$ is $8 \%$. Across all the yearly aggregated matrices of step 9 ), this final $T^{*}$ to $T$ transition matrix is based upon 285 observations. As not all the storms have forecast data going back to the $\left(T^{*}-11\right)$ period, the lowest total number of observations used to construct a transition probability matrix is 195 , for the $\left(T^{*}-11\right)$ period.

Table IV.11. Markov Transition Matrix for $T^{*}$ to $T$

\begin{tabular}{|c|c|c|c|c|c|c|}
\hline $\mathbf{T} \backslash \mathbf{T}$ & $\mathbf{0}$ & $\mathbf{1}$ & $\mathbf{2}$ & $\mathbf{3}$ & $\mathbf{4}$ & $\mathbf{5}$ \\
\hline $\mathbf{0}$ & $100 \%$ & $0 \%$ & $0 \%$ & $0 \%$ & $0 \%$ & $0 \%$ \\
\hline $\mathbf{0 - 0 . 5}$ & $97 \%$ & $3 \%$ & $0 \%$ & $0 \%$ & $0 \%$ & $0 \%$ \\
\hline $\mathbf{0 . 5}-\mathbf{1}$ & $78 \%$ & $22 \%$ & $0 \%$ & $0 \%$ & $0 \%$ & $0 \%$ \\
\hline $\mathbf{1 - 1 . 5}$ & $80 \%$ & $15 \%$ & $5 \%$ & $0 \%$ & $0 \%$ & $0 \%$ \\
\hline $\mathbf{1 . 5}-\mathbf{2}$ & $63 \%$ & $0 \%$ & $25 \%$ & $13 \%$ & $0 \%$ & $0 \%$ \\
\hline $\mathbf{2 - 2 . 5}$ & $67 \%$ & $5 \%$ & $20 \%$ & $8 \%$ & $0 \%$ & $0 \%$ \\
\hline $\mathbf{2 . 5}-\mathbf{3}$ & $22 \%$ & $0 \%$ & $0 \%$ & $78 \%$ & $0 \%$ & $0 \%$ \\
\hline $\mathbf{3 - 3 . 5}$ & $0 \%$ & $0 \%$ & $0 \%$ & $80 \%$ & $20 \%$ & $0 \%$ \\
\hline $\mathbf{3 . 5}-\mathbf{4}$ & $0 \%$ & $0 \%$ & $0 \%$ & $80 \%$ & $20 \%$ & $0 \%$ \\
\hline $\mathbf{4 - 4 . 5}$ & N/A & N/A & N/A & N/A & N/A & N/A \\
\hline $\mathbf{4 . 5}-\mathbf{5}$ & N/A & N/A & N/A & N/A & N/A & N/A \\
\hline
\end{tabular}


Furthermore, notice from Table 11 that values for $T^{*}$ states [4.0-4.5] and [4.5-5.0] are not available. This is due to both data limitations as well as the previously mentioned inherent constraints of the strike probabilities. In fact, as $n$ becomes larger, more states with unavailable and/or undefined values become more persistent in our probability transition matrices. For example, in period $\left(T^{*}-4\right)$ the maximum state value is $[1.0-1.5]$, while for period $\left(T^{*}-11\right)$ the maximum state value is $[0.5-0.75]$

11) After reviewing these final matrices for each period, the matrices for $T^{*}$ to $T$, and $\left(T^{*}-1\right)$ to $T^{*}$ were slightly revised.

The decision to revise these two matrices was made based upon the data limitations stemming from the use of 19 storms. For example, prior to its revision the $T^{*}$ to $T$ transition matrix had the probability of moving from state $[1.0-1.5]$ at $T^{*}$ to state [0] at $T$ being $100 \%$. However, state $[0.5-1.0]$ at $T^{*}$ only transitioned to state [0] at $T$ $78 \%$ of the time, and $22 \%$ of the time to state [1.0]. Likewise, state [1.5 -2$]$ at $T^{*}$ transitioned to state [0] at $T 63 \%$ of the time, state [2.0] $25 \%$ of the time, and state [3.0] the remaining $13 \%$ of the time. Clearly, the $100 \%$ transition of state $[1-1.5]$ at $T^{*}$ to state [0] at $T$ is a limitation of the data, and is therefore modified. The modified transition has state $[1-1.5]$ at $T^{*}$ to state $[0]$ at $T 80 \%$ of the time, state $[1.0] 15 \%$ of the time, and state [2.0] the remaining $5 \%$ of the time. Similar, conservative modifications were done with other identified data limited states. Note, however, that data were not added where previously there was unavailable or undefined data. A complete listing of the probability transition matrices used in the multi-period model is provided in a separate appendix. 


\section{Costs of Evacuation}

As in the two-period model, we still assume that the longer a household waits to evacuate, the more difficult, and hence more expensive evacuation will become such that the costs of evacuation are increasing as $\left(T^{*}-n\right)$ approaches $T$. We use the evacuation costs produced by Whitehead (2003), as well as the evacuation costs/data from a number of post-storm assessments (USACE, 2006b) to derive a household's average costs of evacuation given a CAT 3 storm. From these and other studies, information on the timing of evacuations is then used to determine how the specific aspects of the determined average CAT 3 evacuation costs increase and/or decrease from $\left(T^{*}-n\right)$ to $T$. Finally, data from Lindell et al., (2002) on the predicted increases in the number of cars and associated number of hours to evacuate along the Texas Gulf Coast for CAT 1 to CAT 5 hurricanes is used to estimate the varying levels of average CAT 1 to CAT 5 evacuation costs from our derived average CAT 3 evacuation cost base.

Because we use actual evacuation cost data from a number of previous studies as an input to our multi-period model, the data itself suffers from selection bias. This is because the costs of evacuation are only collected from those households that actually evacuated during the storms. Based upon our model assumptions where households evacuate when $c_{E V_{\left(T^{*}-n\right)}}<E_{\left(T^{*}-n\right)}\left[V\left\{\theta_{\left(T^{*}-n-1\right)} \mid \theta_{\left(T^{*}-n\right)},\left(T^{*}-n-1\right)\right\}\right]$, by only collecting evacuation costs from households that actually evacuated, the costs of evacuation would likely be biased downward. Despite this selection bias issue, we still assume that the existing evacuation cost data are a reasonable input for our multi-period model, but note that a survey that collects evacuation costs from both households that evacuated and 
those that did not (and hence does not suffer from selection bias) is a more appropriate source of evacuation costs as an input to the multi-period model.

Whitehead (2003) separates overall evacuation costs in North Carolina for Hurricane Bonnie into direct costs (food/lodging/entertainment), travel costs $(\$ 0.32$ per mile driven to evacuate), and travel time costs (50\% of wage rate traveling at $45 \mathrm{mph}$ ). From these data, the specific number of miles traveled and time to destination can be determined. Although, not explicitly used in his analysis, lost income costs were given as $\$ 137$ on average. The overall evacuation costs were further segmented by evacuation destination such that direct costs, travel costs, and travel time costs were shown to vary by those that evacuated to a hotel/motel, a shelter, stayed with friends/family, and other. These evacuation costs (converted to 2004 dollars) are shown in Table 12.

Table IV.12. Average Costs of Evacuation (2004 dollars)

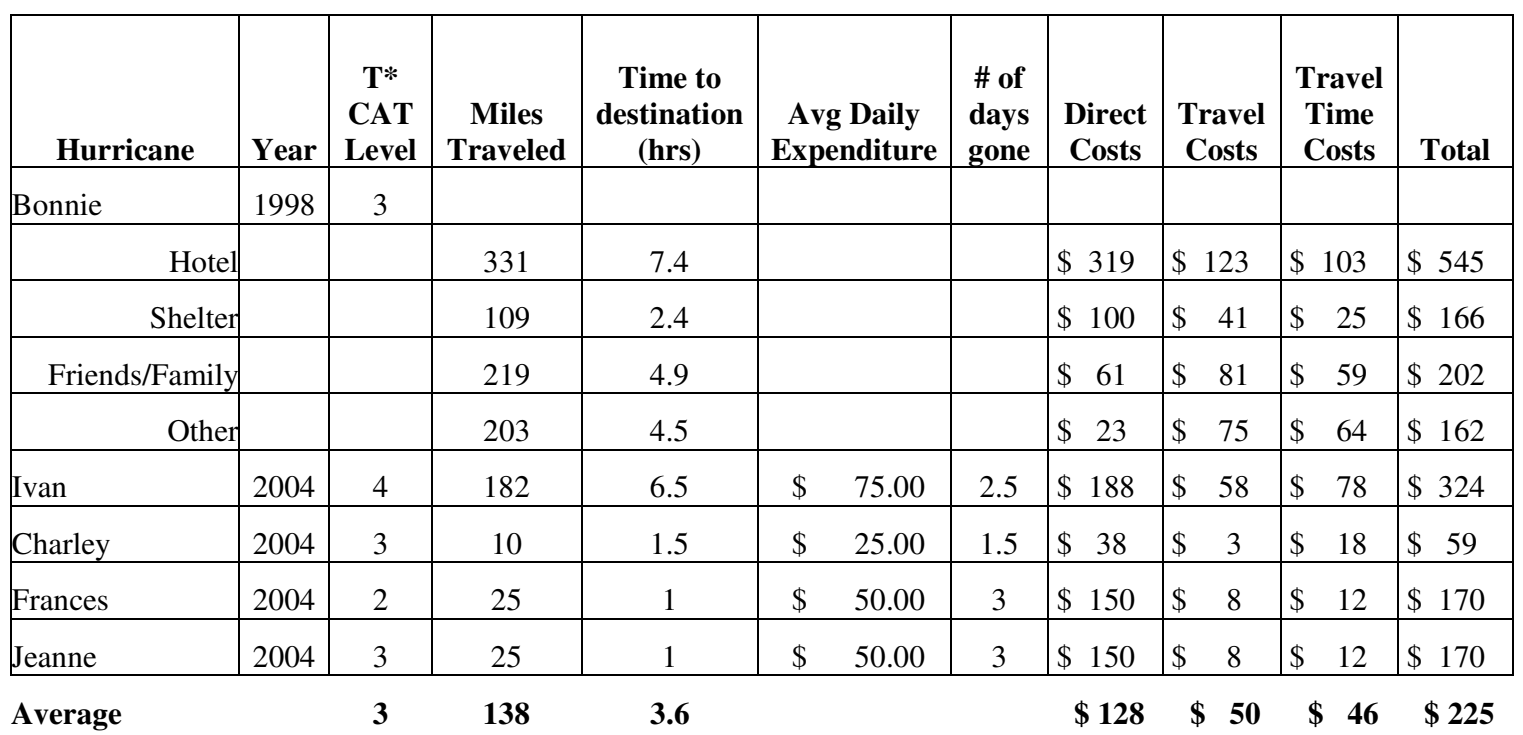

Available evacuation data from USACE (2006b) post-storm assessment studies for 2004 Hurricanes Charley, Frances, Ivan, Jeanne was used to compute similar overall 
evacuation costs ${ }^{56}$. While evacuation costs in these studies were not explicitly given as direct costs, travel time costs, and travel costs, this information is derived from the available data. For Hurricane Ivan, the mean number of miles traveled as well as the average number of hours to reach the final destination are explicitly given as 182 miles and 6.5 hours respectively. For Hurricanes Charley, Frances and Jeanne, the average number of miles traveled, as well as the average number of hours to reach the final destination are determined from graphical evacuation information presented in the reports. For example, graphical information is presented stating that out of 324 Tampa Bay respondents that evacuated for Hurricane Charley, 20\% traveled $>50$ miles, 38\% traveled 11-50 miles, $14 \%$ traveled 6-10 miles, and 28\% traveled 0-5 miles to reach their final destinations. Analogous graphical information is also presented in regard to hours to reach final destination.

The average number of miles traveled was then multiplied by $\$ 0.32$ per mile to estimate the average travel costs for these studies, while the average time to reach the final destination was multiplied by $50 \%$ of hourly wage rate based on annual income of $\$ 50,000$ to estimate the average travel time costs for the 2004 studies. Similarly, for all four 2004 hurricanes, graphical information on the average daily expenditures and the number of days away from home that was presented in the reports was translated into average amounts representing the direct costs of evacuation for the 2004 studies. Table 12 presents the imputed average evacuation costs for the 2004 storms. Compared to the Whitehead (2003) Hurricane Bonnie evacuation costs, the evacuation costs from the 2004

\footnotetext{
${ }^{56}$ While evacuation cost data from Frances and Jeanne was used in the analysis, forecast data from Frances and Jeanne was not used for the construction of the probability transition matrices.
} 
post-storm assessments are higher in regard to direct costs and significantly lower in regard to travel and travel time costs.

From the overall evacuation cost data of Table 12, we determine the average direct, travel, and travel time costs ${ }^{57}$ to be used in the analysis for a CAT 3 storm (which is also the average of the storm CAT level for these five storms). We estimate total evacuation costs for a CAT 3 hurricane to be approximately $\$ 225$, with the majority coming from direct costs. This $\$ 225$ compares to Whitehead's \$316 estimate (in 2004 dollars) for CAT 3 storms. While not as high as Whitehead's estimate, our result is relatively comparable and is importantly derived from more evacuation cost data. In addition to the direct, travel and travel time costs, we also include the lost income costs of $\$ 159$ from the Whitehead study (in 2004 dollars), for total CAT 3 evacuation costs of $\$ 383$.

However, again we are assuming that the overall costs of evacuation are increasing as $\left(T^{*}-n\right)$ approaches $T$. In order to determine how travel, travel time, and portions of direct CAT 3 evacuation costs increase and/or decrease from $\left(T^{\left.*_{-} n\right)}\right.$ to $T$ in our model, data on the cumulative timing of evacuations from existing studies is used. Figures 25 to 32 below illustrate the cumulative evacuation graphs for Hurricanes Bonnie, Ivan, Charley, Frances, Jeanne, Lili, Opal, and Floyd respectively ${ }^{58}$. Using HURREVAC, $T$ and $T^{*}$ are determined for each of these storms and matched up to their

\footnotetext{
${ }^{57}$ Ostensibly, we could also include an additional cost category related to the probability of injury from the actual evacuation, or the chance of not making to your destination before the arrival of the storm, e.g., deaths caused during the Hurricane Rita evacuation in 2005. We abstract from this cost in this study, noting our assumption that $T^{*}$ is the last safe possible period to evacuate.

${ }^{58}$ These storms also average out to a CAT 3 hurricane
} 
cumulative evacuation timing graphs. Given $T$ and $T^{*}$, periods $\left(T^{*}-n\right)$ for $\mathrm{n}=1,2, \ldots$, 11 are then identified as illustrated in the figures below.

Figure IV.25. Cumulative Evacuation Timing Hurricane Bonnie

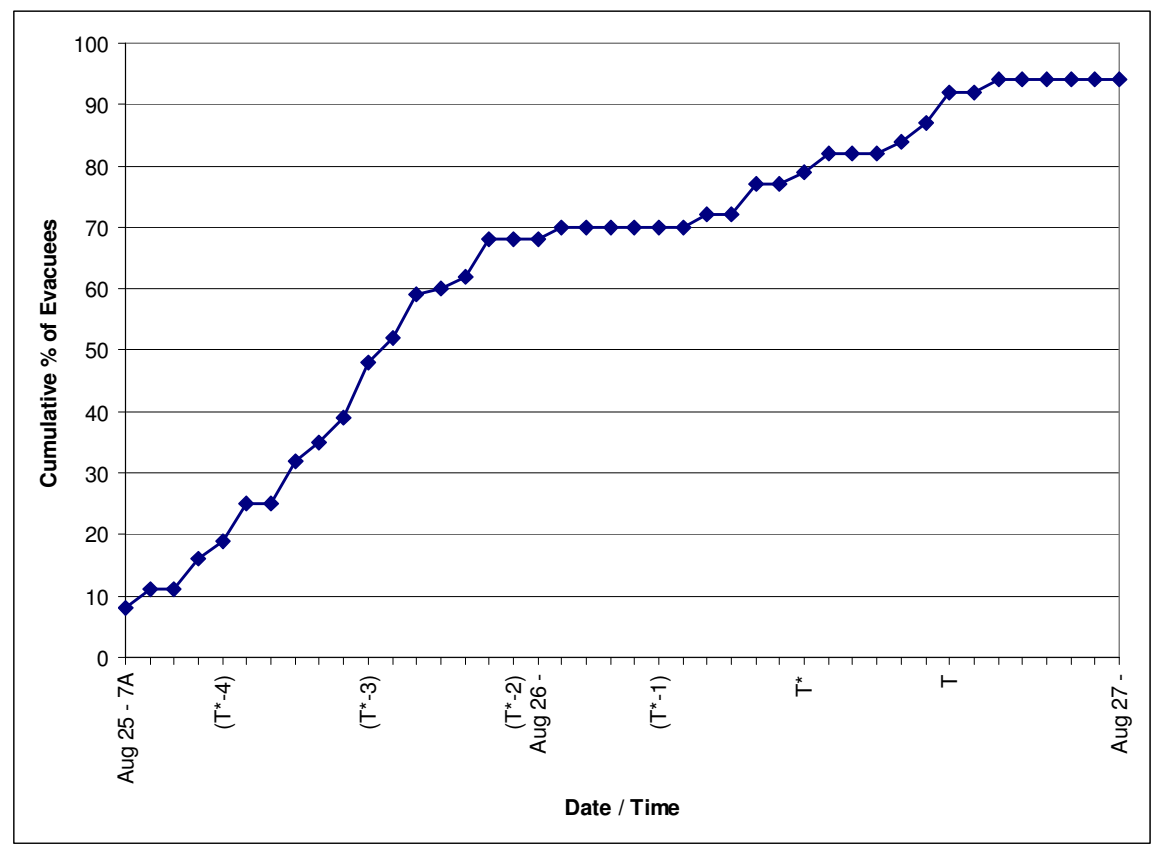

(Source: Adapted from USACE 2006b)

Figure IV.26. Cumulative Evacuation Timing Hurricane Ivan

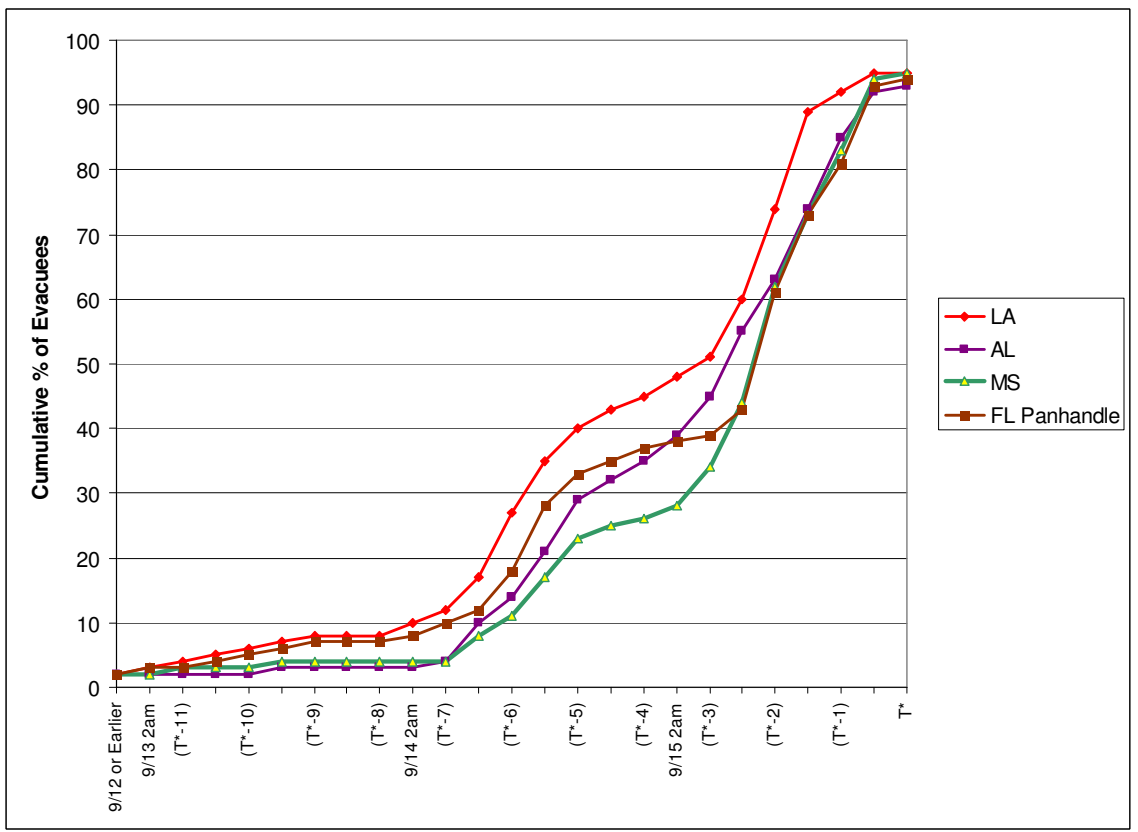

(Source: Adapted from USACE 2006b) 
Figure IV.27. Cumulative Evacuation Timing Hurricane Charley

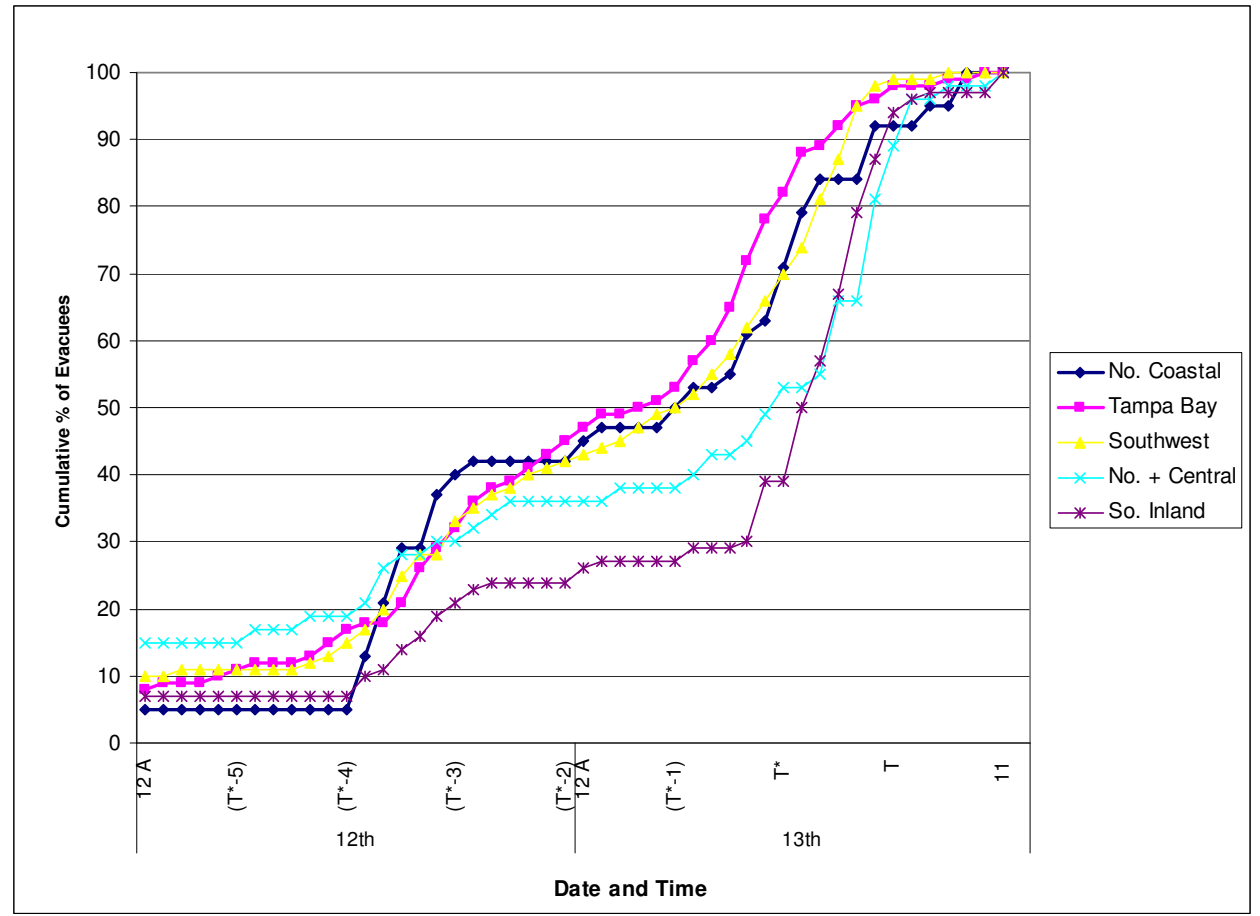

(Source: Adapted from USACE 2006b)

Figure IV.28. Cumulative Evacuation Timing Hurricane Frances

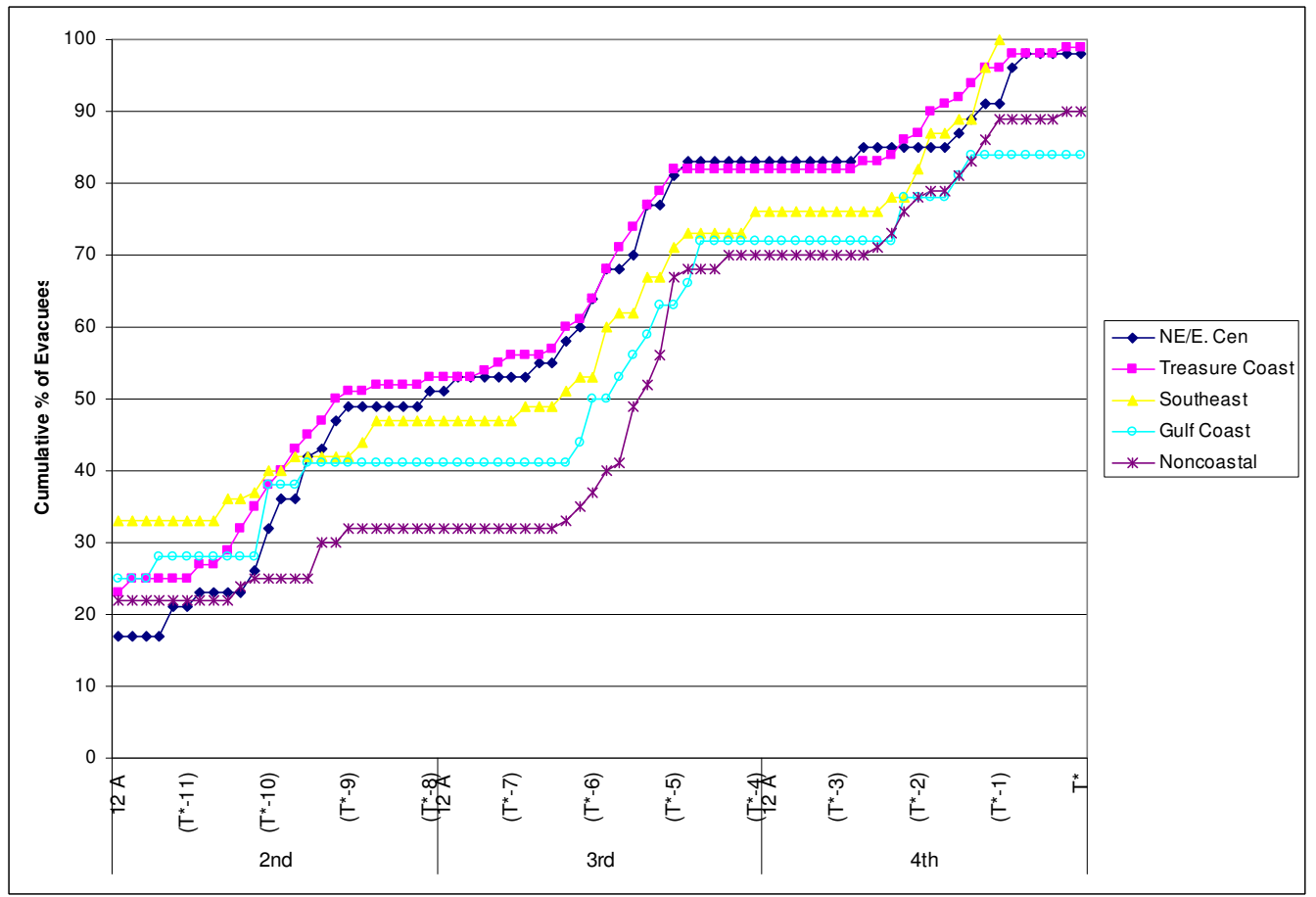

(Source: Adapted from USACE 2006b) 
Figure IV.29. Cumulative Evacuation Timing Hurricane Jeanne

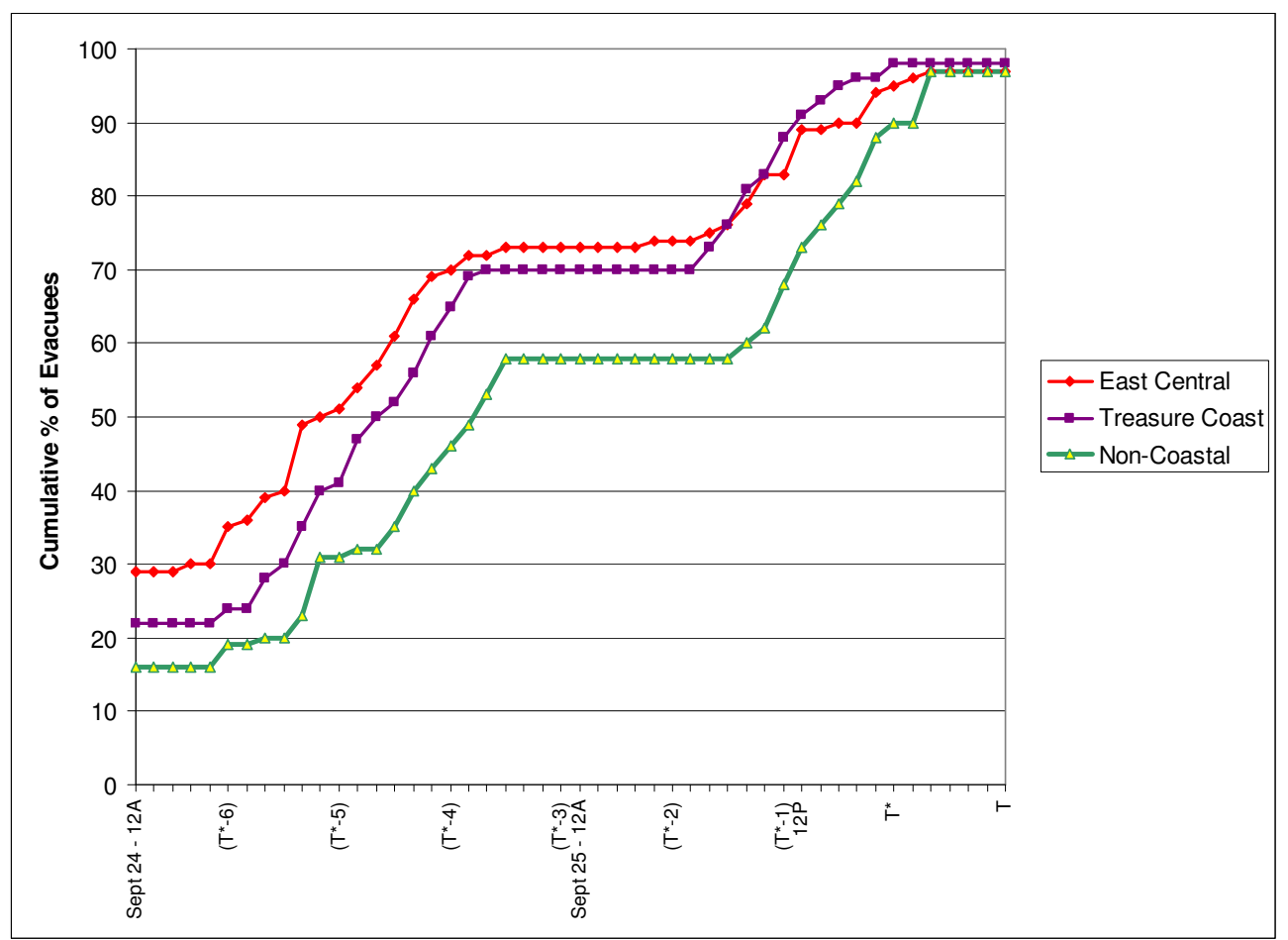

(Source: Adapted from USACE 2006b)

Figure IV.30. Cumulative Evacuation Timing Hurricane Lili

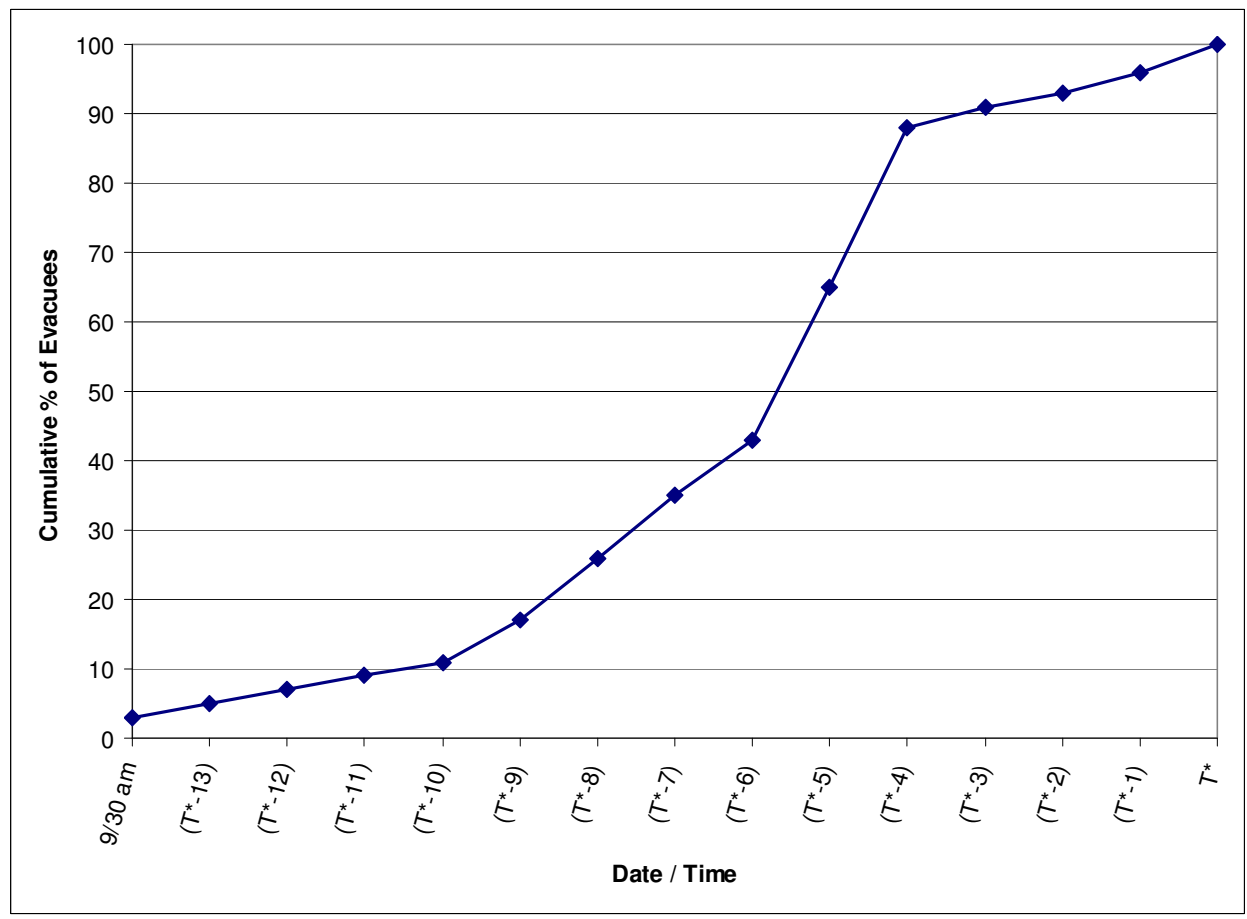

(Source: Adapted from USACE 2006b) 
Figure IV.31. Cumulative Evacuation Timing Hurricane Opal

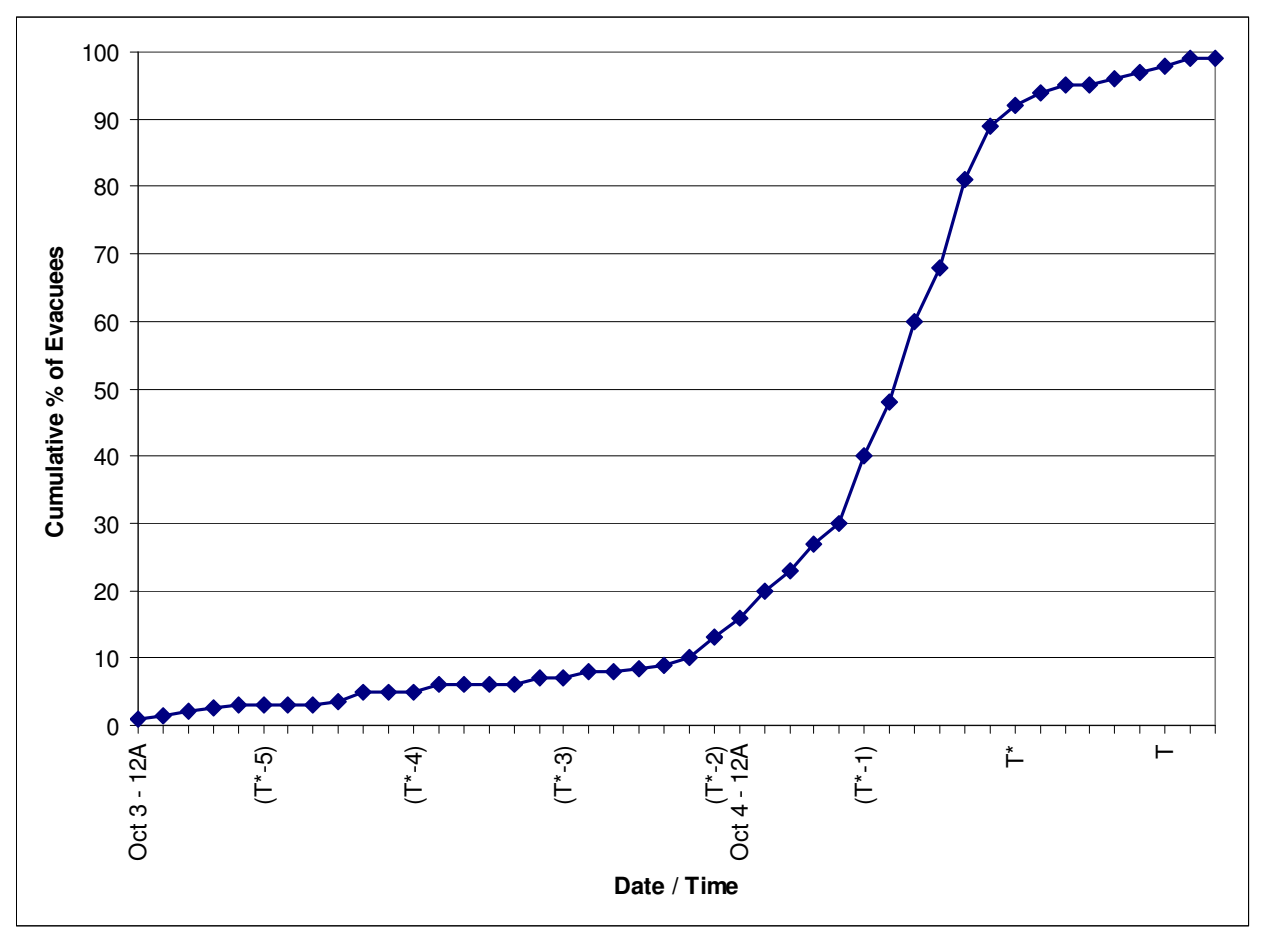

(Source: Adapted from USACE 2006b)

Figure IV.32. Cumulative Evacuation Timing Hurricane Floyd

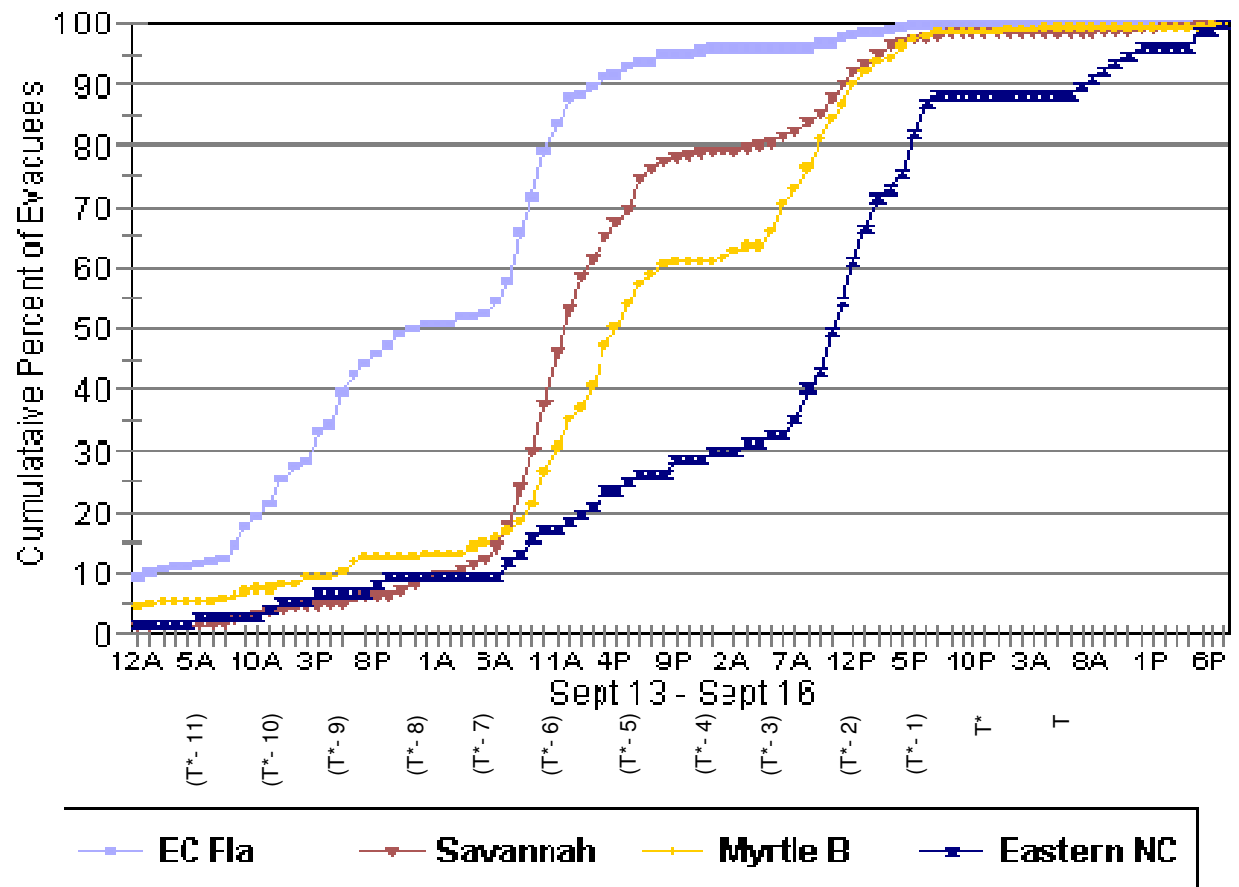

(Source: USACE 2006b) 
For periods $\left(T^{*}-11\right)$ to $T^{*}$, the average cumulative percentage of evacuees across all storms is determined from the graphs as summarized in Table 13. For Hurricanes Ivan, Charley, Frances, Jeanne, and Floyd where evacuation rates for more than one location are provided, an average evacuation rate across all locations is used. From Table 13, we determine and highlight that the $50^{\text {th }}$ percentile of cumulative evacuations is between periods $\left(T^{*}-4\right)$ and $\left(T^{*}-3\right)$, and we therefore assume the $\$ 383$ average CAT 3 costs of evacuation are incurred at this point. That is, if the average costs of evacuating a CAT 3 storm is $\$ 383$, and the average point of evacuating for a CAT 3 storm is $\left(T^{*}-4\right)$, we assume that if a average household evacuates accordingly at period $\left(T^{*}-4\right)$ they incur evacuation costs of $\$ 383$.

Table IV.13. Cumulative Evacuation Timing Summary

\begin{tabular}{|c|c|c|c|c|c|c|c|c|c|c|c|c|}
\hline \multirow[b]{2}{*}{ Hurricane } & \multicolumn{4}{|c|}{ 3rd Day } & \multicolumn{4}{|c|}{ 2nd Day } & \multicolumn{4}{|c|}{ 1st Day } \\
\hline & $\left(T^{*}-11\right)$ & $\left(T^{*}-10\right)$ & $\left(T^{*}-9\right)$ & $\left(T^{*}-8\right)$ & $(\mathrm{T} *-7)$ & $(T *-6)$ & $\left(T^{*-5}\right)$ & $(T *-4)$ & $\left(T^{*-3}\right)$ & $\left(T^{*}-2\right)$ & $\left(T^{*}-1\right)$ & $\mathbf{T}^{*}$ \\
\hline Bonnie & & & & & & & 0.09 & 0.19 & 0.48 & 0.68 & 0.7 & 0.79 \\
\hline Ivan & 0.03 & 0.04 & 0.06 & 0.06 & 0.08 & 0.18 & 0.31 & 0.36 & 0.42 & 0.65 & 0.85 & 0.94 \\
\hline Charley & & & & & & 0.09 & 0.1 & 0.13 & 0.31 & 0.38 & 0.44 & 0.63 \\
\hline Frances & 0.26 & 0.35 & 0.43 & 0.45 & 0.46 & 0.54 & 0.73 & 0.77 & 0.77 & 0.82 & 0.92 & 0.94 \\
\hline Jeanne & & & & & 0.22 & 0.26 & 0.41 & 0.6 & 0.67 & 0.67 & 0.8 & 0.94 \\
\hline Lili & 0.09 & 0.11 & 0.17 & 0.26 & 0.35 & 0.43 & 0.65 & 0.88 & 0.91 & 0.93 & 0.96 & 1 \\
\hline Opal & & & & & & & 0.03 & 0.05 & 0.07 & 0.13 & 0.4 & 0.92 \\
\hline Floyd & 0.05 & 0.07 & 0.1 & 0.12 & 0.15 & 0.3 & 0.55 & 0.61 & 0.65 & 0.88 & 0.95 & 0.98 \\
\hline $\begin{array}{r}\text { Avg \% } \\
\text { Evacuated }\end{array}$ & $11 \%$ & $14 \%$ & $19 \%$ & $22 \%$ & $25 \%$ & $30 \%$ & $36 \%$ & $45 \%$ & $54 \%$ & $64 \%$ & $75 \%$ & $89 \%$ \\
\hline$\%$ Change & & $33 \%$ & $33 \%$ & $17 \%$ & $13 \%$ & $19 \%$ & $20 \%$ & $25 \%$ & $19 \%$ & $20 \%$ & $17 \%$ & $19 \%$ \\
\hline
\end{tabular}

Additionally, from the cumulative evacuation data of Table 13, we determine the percentage change in the average cumulative percentage of evacuees per period. For example, from period $\left(T^{*_{-}} 7\right)$ to $\left(T^{*_{-}} 6\right)$, the average percentage of evacuees increases by 
approximately $20 \%$, going from $25 \%$ in $\left(T^{*}-7\right)$ to $30 \%$ in $\left(T^{*}-6\right)$. This percentage change is then used to dictate how the travel, travel time, and portions of direct costs increase from $\left(T^{*}-11\right)$ to $T^{*}$ based upon the average cost data being incorporated at period $\left(T^{*}-4\right)$. While we assume travel and travel time costs are increasing by these percentages each period over the three days from $\left(T^{*}-11\right)$ to $T^{*}$, we assume direct costs are increasing by these percentages per period only in the last day out from landfall, or periods $\left(T^{*}-3\right)$ to $T^{*}$. Prior to $\left(T^{*}-3\right)$, direct costs are decreasing per day from three days to two days out from landfall. That is, if one were to evacuate three days out from landfall, the direct costs of evacuation incurred at this point such as lodging and food would be greater than if one were to not evacuate three days out from landfall and instead evacuate two days out from landfall. We assume direct costs increase by $50 \%$ per day from $\$ 128$ for evacuation two days from landfall, to $\$ 193$ for evacuation three days from landfall, and also increase each period from $\left(T^{*}-3\right)$ to $T^{*}$ to account for the increased numbers of evacuees.

Similar to direct costs on three and two days out from landfall, we do not assume the $\$ 159$ cost of lost income changing per period, but rather per day, and furthermore we do not assume lost wages ever increasing over time, but always decreasing. Assuming the average $\$ 159$ in lost income is also incurred at period $\left(T^{*}-4\right)$, indicates lost income being incurred two days out from landfall. That is, if one were to evacuate three days from landfall, the lost income costs of evacuation incurred at this point would be greater than if one were to work the three days out from landfall and instead evacuate two days out from landfall. We assume lost income costs of $\$ 159$ for evacuation two days from 
landfall increase by $50 \%$ to $\$ 238$ for evacuation three days from landfall, and decrease by $50 \%$ to $\$ 79$ for evacuation one day from landfall ${ }^{59}$.

Results of the costs increases and decreases by category are presented in Table 14, which illustrates that overall costs of evacuation for a CAT 3 hurricane increasing from $\$ 454$ for evacuation at period $\left(T^{*}-11\right)$ to $\$ 526$ for evacuation at period $T^{*}$. Importantly though, these overall evacuation costs are not increasing linearly. In fact, given our assumption concerning decreasing direct and lost income costs, and the rates of increase for direct, travel, and time costs, we actually see a decline in overall costs between periods $\left(T^{*_{-}} 8\right)$ and $\left(T^{*_{-}} 7\right)$, as well as between periods $\left(T^{*_{-}} 4\right)$ and $\left(T^{*_{-} 3}\right)$.

Table IV.14. Increasing/Decreasing Evacuation Costs

$\underline{\text { CAT } 3} \quad \underline{\left(T^{*}-11\right)}\left(T_{*-10}^{*}\left(T^{*-9}\right)\left(T^{*-8}\right)\left(T^{*-7}\right)\left(T^{*-6}\right)\left(T^{*-5}\right)\left(T^{*-4}\right)\left(T^{*-3}\right)\left(T^{*-2}\right)\left(T^{*-1}\right) T^{*}\right.$

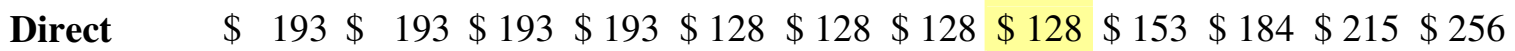

Travel

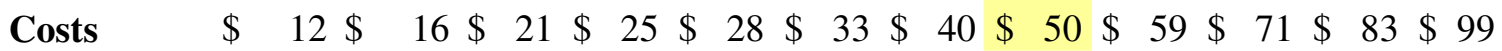

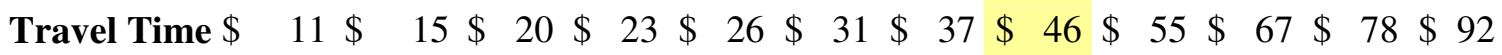

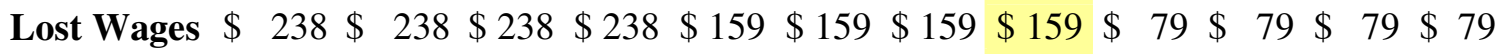

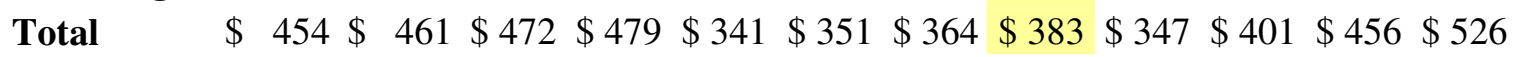

Thus far, the overall increasing costs of evacuation that we have determined are for a CAT 3 hurricane. But we also further assume that these costs would be less for CAT $1 \& 2$ and more for CAT $4 \& 5$ hurricanes because higher CAT levels induce more evacuees. We use data from Lindell et al. (2002) to indicate the increased time to evacuate for CAT 1 to CAT 5 hurricanes. Their data comes from a study of Texas Gulf

\footnotetext{
${ }^{59}$ Given that travel time costs are based on a wage rate, one could make the argument that travel time costs should change in the same way lost income costs do. We focus on increasing travel costs over time and abstract from this.
} 
Coast evacuation response rates. A summary of the estimated time to evacuate (ETE) by county is given in Table 15 .

Table IV.15. Texas Gulf Coast ETEs

$\begin{array}{lccccc}\text { Location } & \mathbf{1} & \mathbf{2} & \underline{\mathbf{3}} & \underline{\mathbf{4}} & \mathbf{5} \\ \text { VSA1 (Cameron South) } & 7 & 8 & 9 & 10 & 11 \\ \text { VSA2 (Cameron North) } & 15 & 21 & 28 & 32 & 33 \\ \text { VSA3 (Willacy) } & 7 & 7 & 7 & 8 & 8 \\ \text { CSA1 (Kenedy/Kleberg) } & 7 & 7 & 8 & 9 & 9 \\ \text { CSA2 (Nueces) } & 14 & 20 & 28 & 31 & 32 \\ \text { CSA3 (Refugio/Aransas) } & 8 & 8 & 8 & 8 & 8 \\ \text { CSA4 (San Patricio) } & 8 & 11 & 15 & 17 & 18 \\ \text { MSA1 (Calhoun/Victoria) } & 8 & 8 & 9 & 10 & 10 \\ \text { MSA2 (Calhoun/Jackson) } & 7 & 8 & 8 & 8 & 8 \\ \text { MSA3 (Matagorda West) } & 7 & 8 & 8 & 9 & 9 \\ \text { MSA4 (Matagorda East) } & 7 & 8 & 8 & 8 & 8 \\ \text { MSA5 (Victoria) } & & 7 & 7 & 7 & 7 \\ \text { GSA1 (Brazoria) } & 7 & 9 & 13 & 15 & 15 \\ \text { GSA2 (Galveston West/Harris South) } & 14 & 20 & 28 & 32 & 33 \\ \text { GSA3 (Harris Central) } & 7 & 7 & 9 & 10 & 10 \\ \text { GSA4 (Harris East) } & 8 & 12 & 17 & 19 & 20 \\ \text { SSA1 (Chambers West) } & 7 & 7 & 7 & 8 & 8 \\ \text { SSA2 (Chambers East/Galveston East) } & 10 & 13 & 17 & 19 & 19 \\ \text { SSA3 (Hardin) } & 7 & 7 & 7 & 7 & 7 \\ \text { SSA4 (Jasper) } & 7 & 7 & 7 & 7 & 7 \\ \text { SSA5 (Jefferson/Orange West) } & 14 & 20 & 29 & 33 & 34 \\ \text { SSA6 (Liberty) } & & 7 & 7 & 7 & 7 \\ \text { SSA7 (Newton) } & & & 7 & 9 & 9 \\ \text { SSA8 (Orange East) } & 7 & 7 & 10 & 11 & 12 \\ & & & & & \\ & 8.4 & 10.0 & 12.2 & 13.5 & 13.9 \\ & & \mathbf{1 9 \%} & \mathbf{2 2 \%} & \mathbf{1 1 \%} & \mathbf{3 \%} \\ & & & & & \end{array}$

\section{ETEs by CAT Level}

The percentage changes in the average ETE from the bottom of Table 15 are used to determine how our direct, travel, and travel time CAT 3 evacuation costs increase and 
decrease respectively $^{60}$. We do not change lost income costs between CAT levels. For example, from Table 15 the average ETE for CAT 3 hurricane $=12.2$ hours, increases by $22 \%$ from the average ETE for a CAT 2 hurricane $=10.0$ hours. Therefore, we generate our CAT 2 direct, travel, and travel time costs of evacuation by decreasing our derived CAT 3 direct, travel, and travel time costs of evacuation by $22 \%$. Similar increases and decreases for CAT 1, 4 and 5 costs are completed based upon the percentage change data from Table 15. Table 16 presents the costs of evacuation by period for each CAT level, and Figure 33 graphically illustrates the results of our cost of evacuation methodology. The difference in evacuation costs are most significant between moving from minor hurricanes (CAT 1\&2) to a major hurricane, and the declines in overall costs for periods $\left(T^{*-7}\right)$ and $\left(T^{*}-3\right)$ are clearly illustrated.

Table IV.16. Derived CAT 1 to CAT 5 Costs of Evacuation

\begin{tabular}{|c|c|c|c|c|c|c|c|c|c|c|}
\hline & \multicolumn{2}{|c|}{ CAT 1} & \multicolumn{2}{|c|}{ CAT 2} & \multicolumn{2}{|c|}{ CAT 3} & \multicolumn{2}{|c|}{ CAT 4} & \multicolumn{2}{|c|}{ CAT 5} \\
\hline $\left.\mathrm{T}^{*}-11\right)$ & $\$$ & 387 & $\$$ & 415 & $\$$ & 454 & $\$$ & 478 & & 485 \\
\hline$T^{*}-10$ & $\$$ & 392 & $\$$ & & & 461 & & 486 & & 93 \\
\hline$T^{*}$-9 & & 399 & $\$$ & 429 & & 472 & & 97 & & 505 \\
\hline " & & 404 & $\$$ & & & 47 & & 05 & & 513 \\
\hline *-7 & & 284 & $\$$ & 308 & & 341 & & 61 & & 367 \\
\hline $\left.\mathrm{T}^{*}-6\right)$ & & 292 & $\$$ & 317 & & 351 & & 73 & & 379 \\
\hline$T^{*}$ & & 300 & $\$$ & 327 & & 364 & & 387 & & 39 \\
\hline$\left.T^{*}-4\right)$ & & 313 & $\$$ & 343 & & 383 & & 408 & & 41 \\
\hline * & & 264 & $\$$ & 29 & & 347 & & 377 & & 3 \\
\hline$*_{\ldots}$ & & 301 & $\$$ & 34 & & 401 & & 436 & & 447 \\
\hline$F_{-1}$ & & 339 & & 388 & & 456 & $\$$ & 497 & & 51 \\
\hline & & 387 & & 446 & & 526 & & 575 & & \\
\hline
\end{tabular}

\footnotetext{
${ }^{60}$ While we are changing our evacuation cost amounts by the ETE percentage changes, we are not affecting the percentage change in the average cumulative percentage of evacuees per period at the bottom of Table 13 which we used to determine how travel costs and portions of direct costs increased per period. One could argue that the different storm CAT levels would affect these rates of costs increases per period. However, we do not have data at this level of specificity to incorporate these changes.
} 
Figure IV.33. Derived CAT 1 to CAT 5 Costs of Evacuation

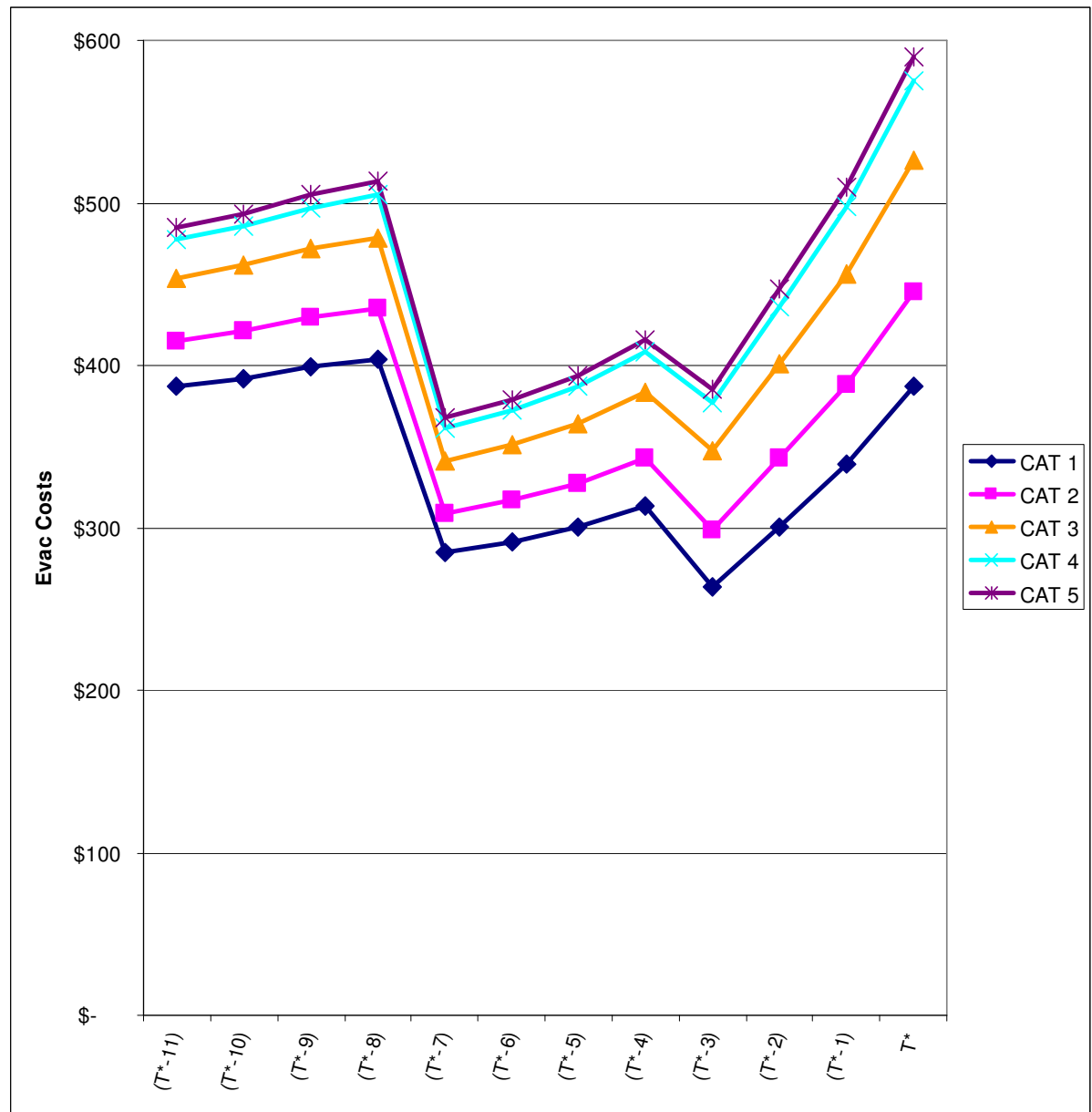

Finally, the derived costs of evacuation for all periods need to be modified in order to coincide with the defined state levels. For example, the costs of evacuating in period $T^{*}, c_{E V_{T^{*}}}$, have been derived for SSHS CAT levels 1 to 5 as provided in Table 16. However, in period $T^{*}$ we have states $0,[0-0.5],[0.5-1],[1.0-1.5], \ldots,[4.5-5.0]$, and therefore $c_{E V_{T^{*}}}$ from Table 16 need to be modified in order to coincide with the identified $T^{*}$ states. CAT 0 costs of evacuation are assumed for all periods to be $75 \%$ of CAT 1 costs, which for $T^{*}$ therefore $=\$ 290$, and costs for states [0-0.5], [1.0-1.5], etc are simply the average of the SSHS CAT level costs above and below them respectively. For 
example, the CAT 1 evacuation costs of $\$ 387$ are associated with the [0.5-1.0] $T^{*}$ state, and the CAT 0 evacuation costs are \$290. Therefore, the evacuation costs for the [0-0.5] $T^{*}$ state are the average of these two values, or $\$ 339$. Similar averaging of the CAT 1 through 5 evacuation costs is completed for all other periods.

\section{Expected Costs of Not Evacuating (Personal Hurricane Damage)}

If a household chooses not to evacuate at $T^{*}$, they will be forced to ride out the storm given that the hurricane ultimately makes landfall at their location. The decision to ride out the storm has an associated probability of being injured, or even killed, depending on the magnitude of the hurricane. We use existing data from the Multihazard Mitigation Council's study to assess the future savings from mitigation activities (MMC, 2005) to assign these probabilities for CAT 1 to CAT 5 hurricanes, and estimate the expected costs of not evacuating from a hurricane (or the value of avoided injury/death).

Similar to the evacuation cost data used, the expected costs of not evacuating from a hurricane also suffer from selection bias. This is because the expected costs of not evacuating are determined based upon injury rates from those households that did not evacuate during a particular hurricane. Assume that for households that had actually evacuated, they instead did not. One would then expect that rates of injuries would go up significantly if all households were present at hurricane landfall. Therefore, by only collecting injury rates from households that did not evacuate, the injury rates, and hence the expected costs of not evacuating would likely be biased downward. Despite this selection bias issue, we still assume that the existing expected cost of not evacuating data is a reasonable input for our multi-period model, but note that a survey that collects the 
probability of being injured during a hurricane from both households that evacuated and those that did not (and hence does not suffer from selection bias) is a more appropriate source for determining the expected costs of not evacuating as an input to the multiperiod model.

The purpose of the MMC study was to quantify the expected benefits of avoided hazard induced losses stemming from natural disasters such as floods, hurricanes, tornadoes, and earthquakes in order to conduct cost-benefit analyses on a number of related mitigation programs. As part of the study, cost of injury data ${ }^{61}$ as well as rates of injury statistics due to hurricanes were collected. We use both of these pieces of information to generate our costs of not evacuating. Table 17 presents the cost of injuries used in their study (converted to 2004 dollars) ranging from minor to critical injuries. Actual hurricane injury rates for three hurricanes were provided in the report: Andrew (CAT 3 in LA) - 0.2\%; Opal (CAT 3) - 0.0\%; and Isabel (CAT 2) - 0.9\%.

Table IV.17. Cost of Injuries (Source MMC)

\begin{tabular}{|c|cc|}
\hline Severity & \multicolumn{2}{|c|}{ Cost $\mathbf{( \$ 2 0 0 4 )}$} \\
\hline Minor & $\$$ & 6,303 \\
\hline Moderate & $\$$ & 51,471 \\
\hline Serious & $\$$ & 189,076 \\
\hline Severe & $\$$ & 619,748 \\
\hline Critical & $\$$ & $2,521,008$ \\
\hline
\end{tabular}

From the existing rates of injury, we take a conservative probability of injury for CAT 3 storms to be $0.45 \%$. We further use the fact that damages along the SSHS are generally thought to follow an exponential form, to ascertain our probabilities of injury

\footnotetext{
61 The cost of injury estimates come from another study that estimated costs of injury damages in car accidents
} 
for CAT 1, 2, 4, and 5 hurricanes. Figure 34 illustrates the probability of injury by hurricane CAT level following an exponential form ${ }^{62}$. The generated probabilities of injury from hurricanes are then multiplied by each of the cost of injury values of Table 17 to obtain an expected cost of not evacuating by CAT level ${ }^{63}$. The expected costs of not evacuating are presented in Table 18, with costs ranging from $\$ 1,694$ for a CAT 1 to $\$ 32,182$ for a CAT 5.

Figure IV.34. Probability of Injury by SSHS CAT

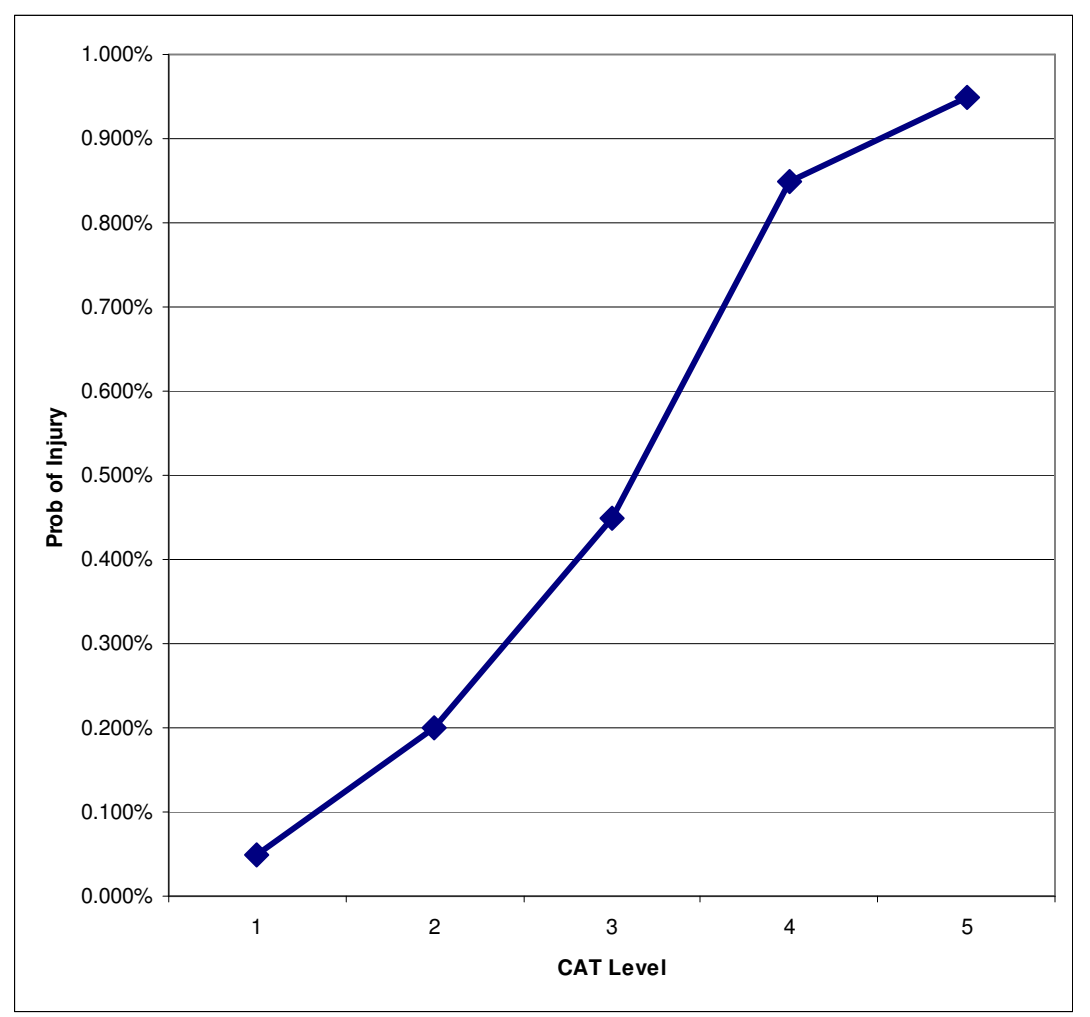

\footnotetext{
${ }^{62}$ The probability of injury between CAT 4 and CAT 5 storms does not increase exponentially

${ }^{63}$ We have assumed that the probability of injury by each CAT level is the same for all types of injuries. For example, in a CAT 3 storm we have assumed the probability of incurring a minor injury is $0.45 \%$, and that the probability of incurring a critical injury is also $0.45 \%$. This is a limitation stemming from our available data.
} 
Table IV.18. Expected Costs of Not Evacuating

\begin{tabular}{|c|c|rr|}
\hline CAT & Prob of injury & \multicolumn{2}{|c|}{$\begin{array}{c}\text { Expected } \\
\text { Cost (\$) }\end{array}$} \\
\hline 0 & $0.000 \%$ & $\$$ & 0 \\
\hline 1 & $0.050 \%$ & $\$$ & 1,694 \\
\hline 2 & $0.200 \%$ & $\$$ & 6,775 \\
\hline 3 & $0.450 \%$ & $\$$ & 15,244 \\
\hline 4 & $0.850 \%$ & $\$$ & 28,795 \\
\hline 5 & $0.950 \%$ & $\$$ & 32,182 \\
\hline
\end{tabular}

The construction of our three main model inputs is ultimately constrained by the available data. Although this is possibly a limitation to the analysis, we believe we have made reasonable assumptions concerning the nature of the inputs, e.g., increasing costs of evacuation as $\left(T^{*}-n\right)$ approaches $T$, and this combined with the fact that all the inputs have been constructed from actual (albeit likely biased) data, allow us to feel comfortable in using the inputs as constructed to draw initial results from our model.

\section{IV.VI. Solution and Results}

From our model setup we have a stochastic, finite-horizon, discrete time, discrete space, Markov decision model which is solved through backward recursion. In time period $T^{*}$, the last safe possible time to evacuate, (29) is $V\left(\theta_{T^{*}}\right)=\min \left\{c_{E V_{T^{*}}}, E_{T^{*}}\left[V\left(\theta_{T}\right) \mid \theta_{T^{*}}\right]\right\}$ with $E_{T^{*}}\left[V\left(\theta_{T}\right) \mid \theta_{T^{*}}\right]=P_{T \mid T^{*}} * E_{T^{*}}\left[c\left(\theta_{T}\right)_{N_{-} E V} \mid \theta_{T^{*}}\right]$, and $P_{T \mid T^{*}}$ being the Markov transition matrix of $\theta$ from $T^{*}$ to $T$. That is, at $T^{*}$ a household does not have the possibility of waiting for a revised hurricane forecast. The choice at $T^{*}$ is either to evacuate and incur $c_{E V_{T^{*}}}$, or to ride the storm out with expected damage equal to $P_{T \mid T^{*}} * E_{T^{*}}\left[c\left(\theta_{T}\right)_{N_{-} E V} \mid \theta_{T^{*}}\right]$. Once $V\left(\theta_{T^{*}}\right)$ has been solved for, 
$V\left(\theta_{T^{*}-1}\right)=\min \left\{c_{E V_{T^{*-1}}}, P_{T^{*}\left(T^{*}-1\right)} *\left[V\left(\theta_{T^{*}}\right) \mid \theta_{\left.T^{* * 1}-1\right)}\right]\right\}$ can be obtained by substituting in $V\left(\theta_{T^{*}}\right)$, and with $P_{T^{*}\left(T^{*}-1\right)}$ being the Markov transition matrix of $\theta$ from $\left(T^{*}-1\right)$ to $T^{*}$.

Similarly, this recursive solution process continues for $V\left(\theta_{T^{*-2}-}\right), \ldots, V\left(\theta_{T^{*-1}}\right)^{64}$.

Table 19 presents the results from (29) for $T^{*}$ where $V\left(\theta_{T^{*}}\right)=\min \left\{c_{E V_{T^{*}}}, E_{T^{*}}\left[V\left(\theta_{T}\right) \mid \theta_{T^{*}}\right]\right\}$, and where the Section $\mathrm{V}$ values from Table 11, Table 18, and Table 16 are substituted for $P_{T T^{*}}, c\left(\theta_{T}\right)_{N_{-} E V} \mid \theta_{T^{*}}$, and $c_{E V_{T^{*}}}$ respectively.

Table 19 indicates that at $T^{*}$ it is optimal for an average household in our Gulf of Mexico region to evacuate for storms with a risk index $>1.0$. Note that transition matrix data for storms with risk indices $>4.0$ in period $T^{*}$ are not available from the storm forecast data used, coinciding with an "N/A" value in Table 19.

Table IV.19. $V\left(\theta_{T^{*}}\right)=\min \left\{c_{E V_{T^{*}}}, E_{T^{*}}\left[V\left(\theta_{T}\right) \mid \theta_{T^{*}}\right]\right\}$

\begin{tabular}{|c|c|c|c|c|}
\hline$\theta_{T^{*}}$ & $c_{E V_{T^{*}}}$ & $E_{T^{*}}\left[V\left(\theta_{T}\right) \mid \theta_{T^{*}}\right]$ & $\begin{array}{c}\text { Optimal } \\
\text { Decision }\end{array}$ & $V\left(\theta_{T^{*}}\right)$ \\
\hline 0 & 290 & 0 & Wait & 0 \\
\hline $0-0.5$ & 339 & 47 & Wait & 47 \\
\hline $0.5-1$ & 387 & 368 & Wait & 368 \\
\hline $1-1.5$ & 416 & 593 & Evacuate & 416 \\
\hline $1.5-2$ & 446 & 3599 & Evacuate & 446 \\
\hline $2-2.5$ & 486 & 2659 & Evacuate & 486 \\
\hline $2.5-3$ & 526 & 11857 & Evacuate & 526 \\
\hline $3-3.5$ & 551 & 17954 & Evacuate & 551 \\
\hline $3.5-4$ & 575 & 17954 & Evacuate & 575 \\
\hline $4-4.5$ & 583 & N/A & N/A & N/A \\
\hline $4.5-5$ & 590 & N/A & N/A & N/A \\
\hline
\end{tabular}

${ }^{64}$ While we collect data over 19 periods, we only use the data up to the $11^{\text {th }}$ period in solving the model. 
Likewise, Table 20 presents the results from (29) for period $\left(T^{*}-1\right)$, where $V\left(\theta_{T^{*}-1}\right)=\min \left\{c_{E V_{T^{*-1}}}, P_{T^{*}\left(T^{*}-1\right)} *\left[V\left(\theta_{T^{*}}\right) \mid \theta_{\left(T^{*}-1\right)}\right]\right\}$, and where $V\left(\theta_{T^{*}}\right)$ are the values from the last column of Table 19, $P_{T^{*}\left(T^{*}-1\right)}$ is the Markov transition matrix of $\theta$ from $\left(T^{*}-1\right)$ to $T^{*}$, and $c_{E V_{T^{*-1}}}$ are the Section $\mathrm{V}$ values from Table 16. At $\left(T^{*}-1\right)$, it is also optimal for an average household to evacuate for storms with a risk index $>1.0$. However, note that a risk index of 1.0 in period $\left(T^{*}-1\right)$ does not carry an equivalent risk connotation as a risk index of 1.0 in period $T^{*}$. Again, note that transition matrix data for storms with risk indices $>3.0$ in period $\left(T^{*}-1\right)$ are not available from the storm forecast data used, coinciding with an "N/A" value in Table 20.

Table IV.20. $V\left(\theta_{T^{*}-1}\right)=\min \left\{c_{E V_{T^{*-1}}}, P_{T^{*}\left(T^{*}-1\right)} *\left[V\left(\theta_{T^{*}}\right) \mid \theta_{\left(T^{*}-1\right)}\right]\right\}$

\begin{tabular}{|c|c|c|c|c|}
\hline$\theta_{\left(T^{*}-1\right)}$ & $c_{E V_{\left(T^{*}-1\right)}}$ & $E_{\left(T^{*}-1\right)}\left[V\left(\theta_{T^{*}}\right) \mid \theta_{\left(T^{*}-1\right)}\right]$ & $\begin{array}{c}\text { Optimal } \\
\text { Decision }\end{array}$ & $V\left(\theta_{\left(T^{*}-1\right)}\right)$ \\
\hline 0 & 254 & 1 & Wait & 1 \\
\hline $0-0.5$ & 296 & 40 & Wait & 40 \\
\hline $0.5-1$ & 339 & 246 & Wait & 246 \\
\hline $1-1.5$ & 363 & 410 & Evacuate & 363 \\
\hline $1.5-2$ & 388 & 454 & Evacuate & 388 \\
\hline $2-2.5$ & 422 & 495 & Evacuate & 422 \\
\hline $2.5-3$ & 456 & 533 & Evacuate & 456 \\
\hline $3-3.5$ & 477 & N/A & N/A & N/A \\
\hline $3.5-4$ & 497 & N/A & N/A & N/A \\
\hline $4-4.5$ & 504 & N/A & N/A & N/A \\
\hline $4.5-5$ & 510 & N/A & N/A & N/A \\
\hline
\end{tabular}

Similarly, this recursive solution process continues for $V\left(\theta_{T^{*}-2}\right), \ldots, V\left(\theta_{T^{*}-11}\right)$. A complete listing of the dynamic programming results for each $\left(\mathrm{T}^{*}-\mathrm{n}\right)$ period are provided in a separate appendix. 
Figure 35 presents the $\theta_{\left(T^{*}-n\right)}$ evacuation cut-off results for all $\left(T^{*}-n\right)$ periods, $n=$ $0,1, \ldots, 11$, along with the maximum risk index determined for each of these periods. For periods $T^{*}$ to $\left(T^{*-2}\right)$ we see that it is rational for an average household at a representative Gulf of Mexico location to evacuate when the forecasted hurricane risk index is $>1.0$, and for period $\left(T^{*}-3\right)$ when $>0.75$. This corresponds to the indicated evacuation region in Figure 35. However, for storms with risk indices $<1.0$ in periods $T^{*}$ to $\left(T^{*}-2\right),<0.75$ in period $\left(T^{*}-3\right)$, and for any determined risk index values beyond period $\left(T^{*}-3\right)$ our model indicates that it is not rational to evacuate. This corresponds to the indicated waiting region of Figure 35.

Figure IV.35. Average Household Optimal Evacuation Results

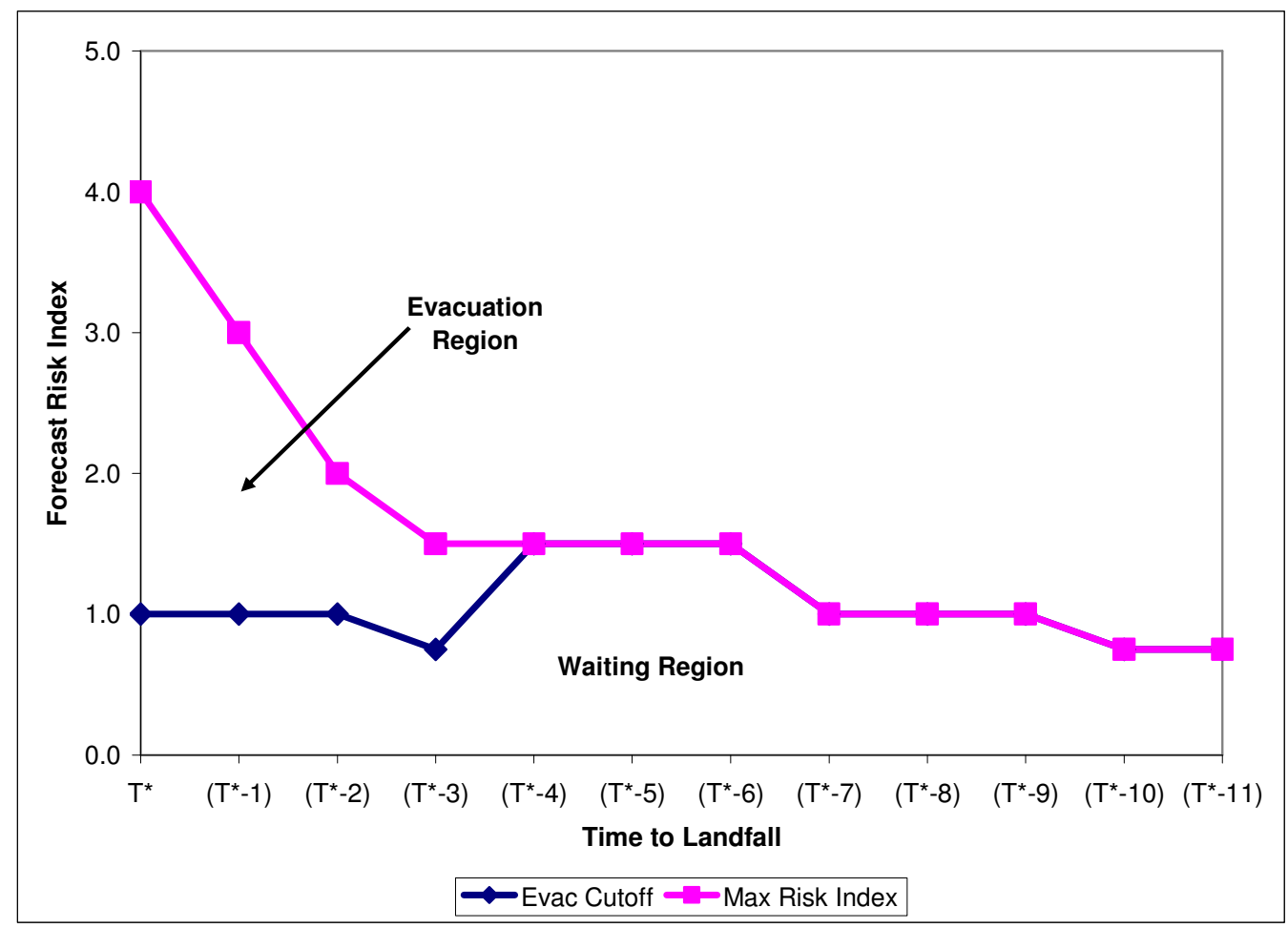


While our results thus far have been general, i.e., for an average household at an representative location in our defined Gulf of Mexico region, we can use the applicable evacuation timing graphs from Figures 25 to 32 in Section V, as well as our per period, per location constructed risk indices to evaluate how well our model does in explaining actual evacuation timing outcomes. We analyze four storms for which we have evacuation timing information, and whose forecast data were included in our probability transition matrices - Hurricanes Ivan, Opal, Charley, and Lili:

\section{$\underline{\text { Hurricane Ivan }}$}

In September, 2004 Hurricane Ivan made landfall as a strong CAT 3, borderline CAT 4 hurricane over Mobile, AL and Pensacola, FL. Figure 26 illustrates the evacuation timing for evacuees from LA (Jefferson, Orleans, Plaquemines, St. Bernard, St. Charles, St. John, St. Tammany Parishes), AL (Baldwin and Mobile counties), MS (Hancock, Harrison, Jackson counties), and FL (Bay, Escambia, Franklin, Gulf, Inland counties, Okaloosa, Santa Rosa, and Walton counties) from the 2004 post-storm assessment report (USACE, 2006b). Depending upon the state, Figure 26 shows the $50^{\text {th }}$ percentile evacuee leaving during either period $\left(T^{*_{-}} 3\right)$ or $\left(T^{*_{-}} 2\right)$. Forecasts for Ivan from periods $\left(T^{*}-10\right)$ to $T^{*}$ consistently called for a CAT 4 hurricane at landfall.

Table 21 presents the per period risk indices by location, ranked in descending order by $T^{*}$, for Hurricane Ivan. Following from the general results of our multi-period model, evacuation is rational beginning in period $\left(T^{*}-3\right)$ for average households in our defined locations of Mobile (Mobile County), AL, Pensacola (Escambia County) and 
Panama City (Bay County), FL, Gulfport (Harrison County), MS, and Buras (Plaquemines Parish), LA, as risk indices for these locations are all $>0.75$ and are highlighted in Table 21. Therefore, for Hurricane Ivan our predicted results for these locations coincide fairly well with the actual evacuation timing illustrated in Figure 26, where the $50^{\text {th }}$ percentile of evacuees as well as the steepest slopes of the evacuation timing curves are occurring in periods $\left(T^{*_{-}} 3\right)$ to $\left(T^{*_{-}} 1\right)$. However, the evacuation occurring for Ivan during periods $\left(T^{*_{-}} 7\right)$ to $\left(T^{*_{-}} 4\right)$ is not predicted from our model - at least not for an average household.

Table IV.21. Hurricane Ivan Risk Indices by Location

$\underline{\text { Evac for: }}>1>1>1>.75$ Wait Wait Wait Wait Wait Wait Wait Wait

\begin{tabular}{|c|c|c|c|c|c|c|c|c|c|c|c|c|c|c|}
\hline State & Locations & Ivan & I & & & & $\mathbf{v}_{-}$ & $\mathbf{v}_{-}$ & {$\left[\mathbf{v}_{-}\right.$} & $\mathbf{v}_{-}$ & Iv_t & Iv_ & Iv_t & Iv_t -11 \\
\hline $\mathrm{AL}$ & Mobile & 3.00 & 2.98 & 2.12 & 1.55 & 1.22 & 1.24 & 0.97 & 0.90 & 0.85 & 0.63 & 0.71 & 0.56 & 0.42 \\
\hline $\mathrm{FL}$ & Pensacola & 3.00 & 2.66 & 1.62 & 1.37 & 1.14 & 1.08 & 0.90 & 0.79 & 0.78 & 0.59 & 0.71 & 0.56 & 0.42 \\
\hline MS & Gulfport & 0.00 & 2.18 & 1.87 & 1.40 & 1.14 & 1.24 & 0.97 & 0.94 & 0.85 & 0.67 & 0.74 & 0.59 & 0.42 \\
\hline LA & Buras & 0.00 & 0.98 & 1.44 & 1.37 & 1.10 & 1.28 & 1.01 & 1.01 & 0.93 & 0.74 & 0.78 & 0.63 & 0.48 \\
\hline LA & New Orleans & 0.00 & 0.22 & 0.58 & 0.72 & 0.80 & 1.04 & 0.82 & 0.86 & 0.82 & 0.67 & 0.67 & 0.59 & 0.42 \\
\hline FL & Panama City & 0.00 & 0.36 & 0.54 & 0.68 & 0.80 & 0.72 & 0.67 & 0.60 & 0.59 & 0.52 & 0.63 & 0.52 & 0.39 \\
\hline $\mathrm{FL}$ & Apalachicola & 0.00 & 0.25 & 0.40 & 0.50 & 0.65 & 0.60 & 0.56 & 0.49 & 0.52 & 0.45 & 0.56 & 0.48 & 0.36 \\
\hline FL & St. Marks & 0.00 & 0.29 & 0.40 & 0.40 & 0.53 & 0.48 & 0.45 & 0.41 & 0.45 & 0.37 & 0.48 & 0.41 & 0.30 \\
\hline $\mathrm{FL}$ & Cedar Key & 0.00 & 0.18 & 0.22 & 0.22 & 0.30 & 0.24 & 0.22 & 0.22 & 0.26 & 0.26 & 0.33 & 0.33 & 0.24 \\
\hline FL & Tampa & 0.00 & 0.07 & 0.11 & 0.11 & 0.15 & 0.12 & 0.15 & 0.11 & 0.19 & 0.22 & 0.26 & 0.30 & 0.24 \\
\hline LA & New Iberia & 0.00 & 0.00 & 0.00 & 0.11 & 0.23 & 0.52 & 0.49 & 0.60 & 0.59 & 0.59 & 0.48 & 0.48 & 0.36 \\
\hline TX & Port Arthur & 0.00 & 0.00 & 0.00 & 0.00 & 0.00 & 0.12 & 0.15 & 0.26 & 0.26 & 0.37 & 0.26 & 0.30 & 0.21 \\
\hline TX & Galveston & 0.00 & 0.00 & 0.00 & 0.00 & 0.00 & 0.00 & 0.07 & 0.15 & 0.15 & 0.26 & 0.15 & 0.22 & 0.18 \\
\hline TX & Freeport & 0.00 & 0.00 & 0.00 & 0.00 & 0.00 & 0.00 & 0.00 & 0.07 & 0.07 & 0.19 & 0.11 & 0.15 & 0.15 \\
\hline TX & Port Lavaca & 0.00 & 0.00 & 0.00 & 0.00 & 0.00 & 0.00 & 0.00 & 0.00 & 0.00 & 0.07 & 0.00 & 0.07 & 0.09 \\
\hline
\end{tabular}




\section{Hurricane Opal}

In October, 1995 Hurricane Opal made landfall as a strong CAT 3 hurricane over Pensacola, FL. Figure 31 illustrates the aggregated evacuation timing for evacuees from AL (Baldwin and Mobile counties) and FL (Bay, Escambia, Okaloosa, Santa Rosa, and Walton counties) from the 1995 post-assessment report (USACE, 2006b). Figure 31 shows the $50^{\text {th }}$ percentile evacuee leaving during period $\left(T^{*}-1\right)$, and the slope of the curve steepening considerably over this time period. Forecasts for Opal from periods $\left(T^{*}-11\right)$ to $\left(T^{*}-3\right)$ called for a minor hurricane at landfall, while forecasts from $\left(T^{*}-2\right)$ to $T^{*}$ called for a major hurricane at landfall.

Table 22 presents the per period risk indices by location, ranked in descending order by $T^{*}$, for Hurricane Opal. Following from the general results of our multi-period model, evacuation is rational beginning in period $\left(T^{*}-1\right)$ for average households in our defined locations of Pensacola (Escambia County) and Panama City (Bay County), FL, as well as for Mobile (Mobile County), AL, as risk indices for these locations are all $>1.0$ and are highlighted in Table 22. These results coincide well with the actual evacuation timing as illustrated in Figure 31, where the steepest slope of the evacuation timing curve and the $50^{\text {th }}$ percentile are occurring in period $\left(T^{*}-1\right)$, and overall evacuation is really beginning in earnest during this timeframe. In this case, the results of our multi-period model offer an explanation for the relatively late (12 hours prior to landfall for a major hurricane) evacuation response. 
Table IV.22. Hurricane Opal Risk Indices by Location

\begin{tabular}{|c|c|c|c|c|c|c|c|c|c|c|c|c|c|c|}
\hline & & r: & $>1$ & $>1$ & $>1$ & $>.75$ & it & it & it & it & Vait & Vait & Vait & it \\
\hline tate & Locations & Opal & O_T* & O_T*-1 & O_T ${ }^{*-2}$ & O_T ${ }^{*-3}$ & O_t -4 & O_t -5 & O_t-6 & O_t-7 & O_t-8 & O_t-9 & O_t -10 & O_t-11 \\
\hline $\mathrm{L}$ & Pensacola & & 00 & 14 & 067 & 054 & 040 & 0.32 & 0.30 & 0.22 & 7 & 0.03 & 4 & 0.04 \\
\hline 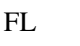 & Panama City & 0.00 & & & & 0 & 0.36 & 0.28 & 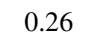 & 2 & 5 & 2 & 0.04 & 03 \\
\hline $\mathrm{L}$ & Mobile & 00 & o & 1.10 & (No & 0.46 & 0.50 & 0.02 & 0.30 & 0.24 & 0.07 & 0.04 & 0.04 & 0.04 \\
\hline L & Apalachic & 00 & 15 & 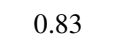 & 07 & 174 & 0.34 & 0.20 & 0.26 & 20 & 0.05 & 0.02 & 0.04 & 0.03 \\
\hline IS & Gulfport & 00 & 0.60 & 0 & 0.49 & 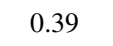 & 0. & 0 & 0 & 4 & 0.09 & 0.04 & 0.05 & 0.05 \\
\hline L & St. Marks & 00 & 0.60 & 0.65 & 0.61 & +9 & 0.30 & 0.24 & 0.24 & 0.18 & 0.04 & 0.00 & 0.03 & 0.02 \\
\hline A & Buras & 0.00 & .28 & 0.76 & 0 & 41 & 0.42 & 0.38 & 0.34 & 0.28 & .12 & 0.06 & 0.07 & 0.06 \\
\hline A & New Orleans & 0.00 & 0.00 & 0.29 & 0.36 & 0.29 & 0.34 & 0.34 & 0.30 & 0.26 & 0.12 & 0.06 & 0.06 & 0.06 \\
\hline FL & Cedar Key & 0.00 & 0.00 & 0.22 & 0.46 & 0.37 & 0.24 & 0.20 & 0.20 & 0.16 & 0.04 & 0.00 & 0.03 & 0.02 \\
\hline FL & Tampa & 0.00 & 0.00 & 0.07 & 0.30 & 0.24 & 0.18 & 0.16 & 0.18 & 0.16 & 0.04 & 0.00 & 0.03 & 0.02 \\
\hline LA & New Iberia & 0.00 & 0.00 & 0.00 & 0.18 & 0.15 & 0.22 & 0.30 & 0.24 & 0.24 & 0.12 & 0.07 & 0.06 & 0.06 \\
\hline TX & Port Arthur & 0.00 & 0.00 & 0.00 & 0.06 & 0.05 & 0.08 & 0.16 & 0.12 & 0.18 & 0.12 & 0.07 & 0.05 & 0.06 \\
\hline TX & Galveston & & & & 0.00 & 0.00 & 0.04 & 0.12 & 0.08 & 0.16 & 0.14 & 0.09 & 0.06 & 0.07 \\
\hline TX & Freeport & 00 & 0.00 & 0.00 & 0.00 & 0.00 & 0.00 & 0.10 & 0.06 & 0.14 & 0.14 & 0.10 & 0.06 & 0.07 \\
\hline$\Gamma \mathrm{X}$ & Port Lavaca & .00 & 0.00 & 0.00 & 0.00 & 0.00 & 0.00 & 0.04 & 0.00 & 0.10 & 0.14 & 0.10 & 0.06 & 0.07 \\
\hline
\end{tabular}

\section{Hurricane Charley}

In August, 2004 Hurricane Charley made landfall in Southwest FL as a CAT 4 hurricane over Punta Gorda. Figure 27 illustrates (amongst other locations) the evacuation timing for evacuees from Northern Coastal FL (Citrus, Dixie, Hernando, Levy, and Taylor counties) and from Tampa Bay Coastal FL (Hillsborough, Manatee, Pasco, and Pinellas counties) from the 2004 post-assessment report (USACE, 2006b). Figure 27 shows the $50^{\text {th }}$ percentile evacuee for these two specific areas leaving during period $\left(T^{*}-1\right)$. Forecasts for Charley from periods $\quad\left(T^{*}-11\right)$ to $\left(T^{*_{-}} 4\right)$ called for a minor hurricane at landfall, while forecasts from $\left(T^{*}-3\right)$ to $T^{*}$ called for a major hurricane at landfall. 
Table 23 presents the per period risk indices by location, ranked in descending order by $T^{*}$, for Hurricane Charley. Following from the general results of our multiperiod model, evacuation is rational beginning in period $\left(T^{*}-3\right)$ for average households in our defined locations of Tampa (Hillsborough County), Cedar Key (Levy County), and St. Marks (Wakulla County) FL, as risk indices for these locations are all $>0.75$ and are highlighted in Table 23. From Figure 27 we see that evacuation timing begins to increase rapidly starting with period $\left(T^{*}-4\right)$, with the slope of the evacuation timing curve increasing significantly between periods $\left(T^{*}-2\right)$ and $T^{*}$. Again, it appears that our model does a good of explaining the timing of the actual evacuation behavior.

Table IV.23. Hurricane Charley Risk Indices by Location

\begin{tabular}{|c|c|c|c|c|c|c|c|c|c|c|c|c|c|c|}
\hline & & & & $>1$ & $>1$ & $>.75$ & & & & & & & it & iit \\
\hline State & Locations & Cha & Ch_T* & Ch_t-1 & Ch_t -2 & Ch_t -3 & Ch_t -4 & Ch_t -5 & $1-t-6$ & Ch_t-7 & -8 & 0 & & $\mathrm{Cb}_{\mathrm{t}}$ \\
\hline FL & Tampa & & 2. & 1.41 & 1.31 & 1.19 & & & & & & & 0.28 & 1 \\
\hline L & Cedar Key & & & 32 & & & & & 4 & 6 & 6 & 7 & 5 & 18 \\
\hline $\mathrm{L}$ & St. Marks & 0 & .00 & 02 & 0 & 0.79 & 0.60 & 0.65 & 0.46 & 46 & 0.29 & 3 & 0 & 0.12 \\
\hline FL & Apalachicola & 00 & 0.39 & 0.84 & 0.46 & 0.61 & 0.50 & 0.58 & 0.36 & 40 & 0.25 & 0.20 & 0.21 & 0.16 \\
\hline FL & Panama City & 0.00 & 0.1 & 0.60 & 024 & 0.43 & 0.36 & 0.43 & 0.28 & 34 & 0.21 & 16 & 0.20 & 0.14 \\
\hline $\mathrm{L}$ & Pensacola & 0.00 & 0.00 & 0.09 & 00 & 0.09 & 0.10 & 0.14 & 0.06 & 14 & 0.07 & 0.07 & 0.12 & 0.09 \\
\hline $\mathrm{AL}$ & Mobile & 00 & 00 & 0.00 & .00 & 0.00 & 0.00 & 0.05 & 0.00 & 0.04 & 0.03 & 0.02 & 0.09 & 0.05 \\
\hline MS & Gulfport & 0.00 & 0.00 & 0.00 & 0.00 & 0.00 & 0.00 & 0.00 & 0.00 & 0.00 & 0.00 & 0.00 & 0.07 & 0.04 \\
\hline $\mathrm{A}$ & Buras & 000 & 00 & 0.00 & 00 & 0.00 & 0.00 & 0.00 & 0.00 & 0.00 & 0.00 & 0.00 & 0.05 & 0.05 \\
\hline LA & New O & 0.00 & 0 . & 0.00 & 0 & 0.00 & 0.00 & 0.00 & 0.00 & 0.00 & 0.00 & 0.00 & 0.04 & 0.00 \\
\hline A & New I & 00 & 0.00 & 0.00 & 0.00 & 0.00 & 0.00 & 0.00 & 0.00 & 0.00 & 0.00 & 0.00 & 0.00 & 0.00 \\
\hline TX & Port Arthur & 0.00 & 0 & 0.00 & م00 & 0.00 & 0.00 & 0.00 & 0.00 & 0.00 & 0.00 & 0.00 & 0.00 & 0.00 \\
\hline $\mathrm{TX}$ & Galveston & 0.00 & 000 & 0.00 & 0. & 0.00 & 0.00 & 0.00 & 0.00 & 0.00 & 0.00 & 0.00 & 0.00 & 0.00 \\
\hline TY & Freeport & a & 0. & 0.00 & 0.00 & 0.00 & 0.00 & 0.00 & 0.00 & 0.00 & 0.00 & 0.00 & 0.00 & 0.00 \\
\hline$\Lambda$ & ort Lavaca & 0.00 & 00 & 0.00 & 00 & 0.00 & 0.00 & 0.00 & 0.00 & 0.00 & 0.00 & 0.00 & 0.00 & 0.00 \\
\hline
\end{tabular}




\section{Hurricane Lili}

In October, 2002 Hurricane Lili made landfall as a CAT 1 hurricane over Vermilion Parish, LA. Figure 30 illustrates the aggregated evacuation timing for evacuees from Louisiana (Cameron, Calcasieu, Jefferson Davis, Vermilion, Acadia, Lafayette, Iberia, St. Mary, St. Martin, Iberville, Terrebonne, Assumption, Lafourche, St. Charles, Jefferson, Plaquemines, Ascension, St. James, and St. John the Baptist parishes) and Texas (Jefferson, Orange, and Chambers counties) from the 2002 post-assessment report (USACE, 2006b). Figure 30 shows the $50^{\text {th }}$ percentile evacuee leaving during period $\left(T^{*}-5\right)$. Forecasts for Lili from periods $\left(T^{*}-11\right)$ to $\left(T^{*}-3\right)$ called for a CAT 3 hurricane at landfall, while forecasts in $\left(T^{*}-2\right)$ and $\left(T^{*}-1\right)$ called for a CAT 4 hurricane at landfall.

Table 24 presents the per period risk indices by location, ranked in descending order by $T^{*}$, for Hurricane Lili. Following from the general results of our multi-period model, evacuation is rational beginning in period $\left(T^{*}-3\right)$ for average households in our defined locations of New Iberia (Iberia Parish) and New Orleans (St. Charles Parish), LA, and Port Arthur (Jefferson County), TX, as risk indices for these locations are all $>0.75$ and are highlighted in Table 24. From Figure 30 we see that evacuation timing begins to increase rapidly starting with period $\left(T^{*_{-}} 7\right)$, with the slope of the evacuation timing curve increasing significantly between periods $\left(T^{*_{-}} 7\right)$ and $\left(T^{*_{-}} 3\right)$. In this case, our model does not do a good job of predicting the beginning of evacuation behavior, but is able to at least capture the tail end of the steepest part of the evacuation timing curve. 
Table IV.24. Hurricane Lili Risk Indices by Location

\begin{tabular}{|c|c|c|c|c|c|c|c|c|c|c|c|c|c|c|}
\hline & & r: & $>1$ & $>1$ & $>1$ & $>.75$ & Wait & Wait & Wait & Wait & Wait & Wait & Wait & iit \\
\hline tate & Locations & Lili & $\mathbf{T}^{*}$ & $T^{*}-1$ & $\mathbf{T}^{*-2}$ & $T^{*-3}$ & -4 & -5 & -6 & -7 & -8 & -9 & -10 & -11 \\
\hline $\mathrm{A}$ & New Iberia & & 2.49 & & 1.48 & 0.96 & 0.79 & 0.70 & 0.64 & 0.55 & 0.51 & 0.45 & 0.36 & 0.39 \\
\hline A & New Orleans & & 0.93 & & 1.00 & 0.76 & 0.64 & 0.58 & 0.55 & 0.48 & 0.48 & 0.42 & 0.33 & 0.36 \\
\hline$\Gamma X$ & Port Arthur & & & & 1.24 & 0.89 & 0.76 & 0.70 & 0.64 & 0.55 & 0.48 & 0.45 & 0.36 & 0.36 \\
\hline MS & Gulfport & 0 & & & 0.76 & 0.60 & 0.49 & 0.42 & 0.39 & 0.39 & 0.42 & 0.36 & 0.27 & 0.30 \\
\hline$A$ & Buras & 0 & 09 & 112 & 0.80 & 0.66 & 0.55 & 0.52 & 0.51 & 0.48 & 0.48 & 0.45 & 0.36 & 0.39 \\
\hline $\mathrm{AL}$ & Mobile & 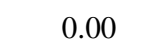 & 09 & 4 & 0.56 & 0.50 & 0.42 & 0.36 & 0.33 & 0.30 & 0.36 & 0.27 & 0.21 & 0.24 \\
\hline $\mathrm{L}$ & Pensacola & . & OQ & 0.08 & 0.24 & 0.33 & 0.33 & 0.27 & 0.24 & 0.24 & 0.30 & 0.24 & 0.18 & 0.24 \\
\hline TX & Galveston & 0.00 & 00 & 0.00 & 0.76 & 0.73 & 0.64 & 0.64 & 0.61 & 0.55 & 0.45 & 0.42 & 0.36 & 0.36 \\
\hline $\mathrm{TX}$ & Freeport & .00 & .00 & & 0.36 & 0.56 & 0.52 & 0.55 & 0.55 & 0.51 & 0.42 & 0.42 & 0.36 & 0.33 \\
\hline FL & Tampa & & .00 & & 0.00 & 0.00 & 0.00 & 0.00 & 0.00 & 0.00 & 0.00 & 0.06 & 0.06 & 0.15 \\
\hline $\mathrm{L}$ & Cedar Key & & & & & 0.00 & 0.00 & 0.00 & 0.00 & 0.00 & 0.00 & 0.06 & 0.06 & 0.12 \\
\hline $\mathrm{L}$ & St. Marks & & & & & & 0.09 & 0.06 & 0.06 & 0.06 & 0.09 & 0.06 & 0.06 & 0.12 \\
\hline $\mathrm{L}$ & Apalachicola & & 0.00 & & 0.00 & 0.10 & 0.09 & 0.09 & 0.06 & 0.09 & 0.15 & 0.12 & 0.12 & 0.18 \\
\hline $\mathrm{L}$ & Panama City & & & & 0.00 & 0.13 & 0.15 & 0.12 & 0.12 & 0.12 & 0.18 & 0.15 & 0.12 & 0.21 \\
\hline TX & Port Lavaca & 0.00 & 0.00 & 0.00 & 0.00 & 0.27 & 0.30 & 0.39 & 0.39 & 0.42 & 0.33 & 0.36 & 0.30 & 0.27 \\
\hline
\end{tabular}

The results from our general multi-period model applied to actual evacuation timing behavior for specific locations and specific storms indicate that our multi-period model does a convincingly good job of predicting evacuation timing outcomes for Gulf of Mexico locations. In all 4 cases, evacuation is predicted only in those locations where actual evacuation occurred according to the post-storm assessment survey data. For example, Hurricane Charley made landfall in Southwest Florida and our model predicts evacuations for locations close to the eventual landfall such as Tampa, while not predicting evacuations for locations not in close proximity such as Mobile, AL. In 3 of the 4 cases, our model correctly predicts evacuation for an average household, which correspond to the $50^{\text {th }}$ percentile on the evacuation timing graphs. Moreover, in the case of Opal our results offer an explanation for the seemingly late evacuation response. 
However, the general results from our multi-period model cannot address the 10$50 \%$ of cumulative evacuations occurring between periods $\left(T^{*}-8\right)$ and $\left(T^{*}-4\right)$ for Hurricanes Lili and Ivan. For both of these hurricanes, forecasts during this period called for a major hurricane strike at landfall. However, recall that the NHC strike probabilities for 36 and 48 hour forecasts are constrained to be less than $25 \%$, which directly affects our risk index calculation and ultimately the evacuation/wait result. Furthermore, our results thus far are for an average household. In addition to evaluating our model against actual evacuation timing outcomes for an average household, we can also evaluate it through expected evacuation outcomes by various evacuation household types.

Let us assume two household types: high damage (coastal location or mobile home) vs. low damage (inland location or non-mobile home), where high (low) damage households have a greater (lower) probability of being injured than those rates presented in Table 18 of Section V. Intuitively, compared to an average damage household type, high (low) damage household types should evacuate earlier (later) in general and also be more (less) willing to evacuate for lower (higher) risk index storms. Figure 36 and 37 present the $\theta_{\left(T^{*-n)}\right.}$ evacuation cut-off results for high damage and low damage household types respectively for all $\left(T^{*}-n\right)$ periods, $n=0,1, \ldots, 11$, along with the maximum risk index determined for each of these periods.

The high damage household optimal evacuation results from our multi-period model shown in Figure 36 coincide with the expected results for this household type. When the probability of injury increases by five times that of the average household, the evacuation region expands for lower risk indices in periods $T^{*}$ to $\left(T^{*}-3\right)$. Furthermore, if the probability of injury has increased significantly, one would also expect the number of 
evacuees to increase leading to higher rates of increase of evacuation costs for all periods compared to those used for an average household as given in Table 13 of Section V. When the higher probability of injury is coupled with evacuation cost increase twice that of an average household, not only does the evacuation region expand for periods $T^{*}$ to $\left(T^{*}-3\right)$, but earlier evacuation is induced for periods $\left(T^{*_{-} 5}\right)$ to $\left(T^{*}-7\right)$. Likewise, the low damage household optimal evacuation results from our multi-period model shown in Figure 37 coincide with the expected results for this household type. When the probability of injury decreases to half of that of the average household, the evacuation region contracts with evacuation optimal for higher risk indices in periods $T^{*}$ to $\left(T^{*-3}\right){ }^{65}$

Figure IV.36. High Damage Household Optimal Evacuation Results

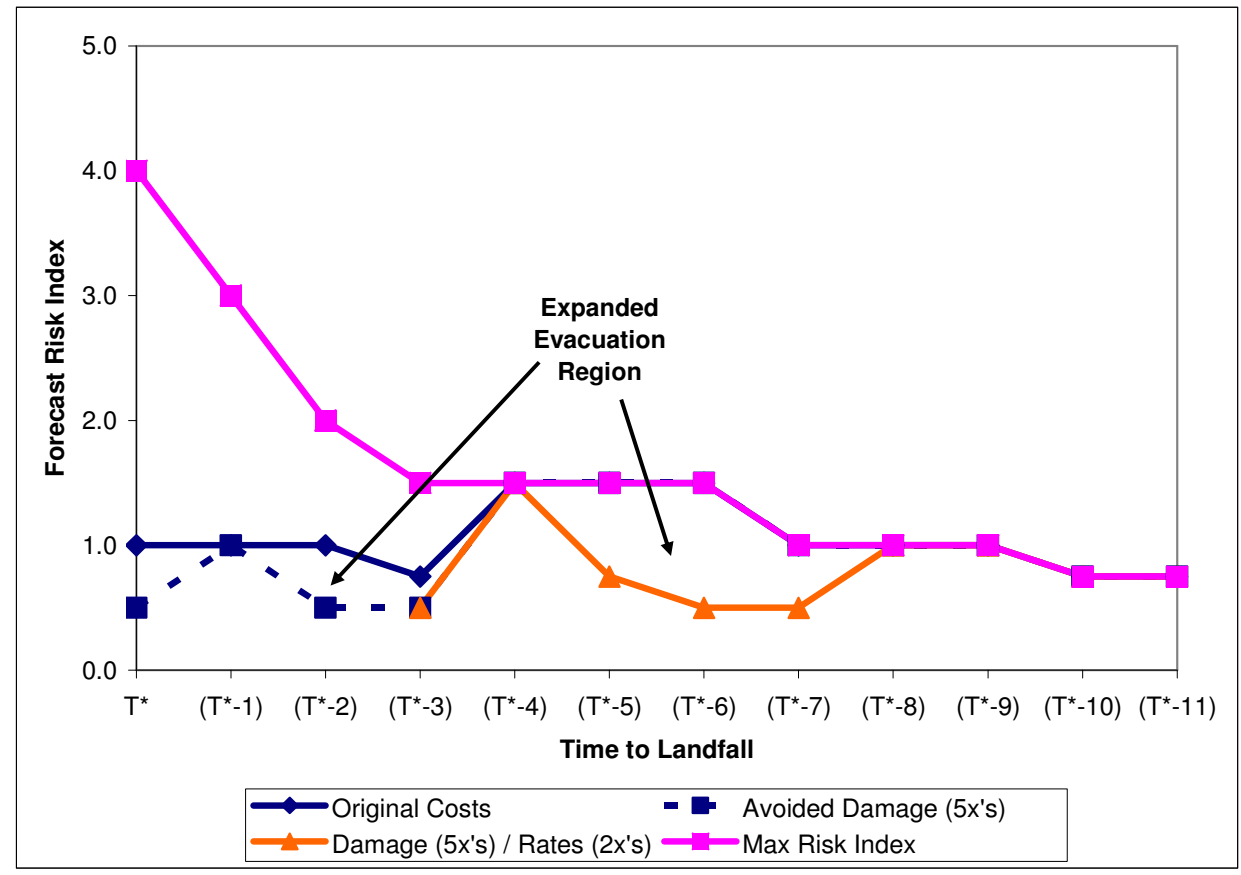

\footnotetext{
${ }^{65}$ Decreasing the rates of costs of evacuation increases only changed results from Figure 37 slightly and are therefore not shown.
} 
Figure IV.37. Low Damage Household Optimal Evacuation Results

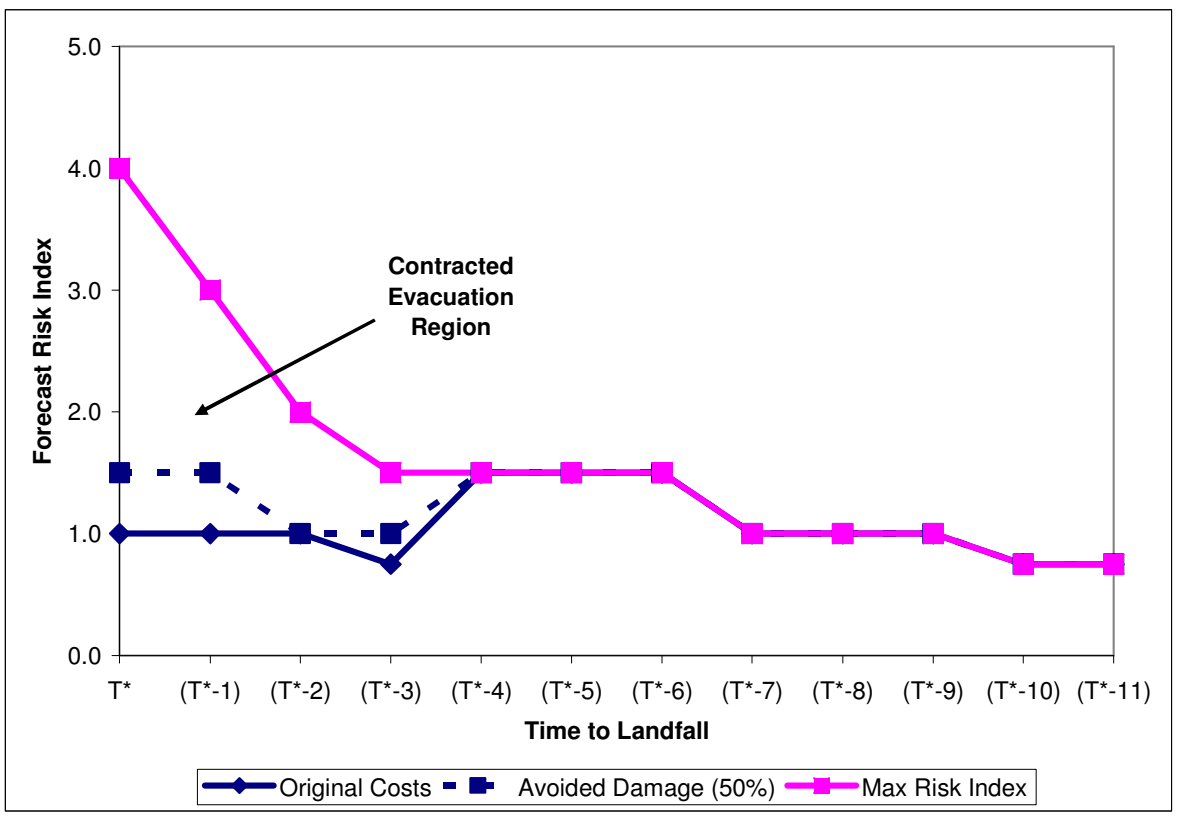

We can also assume household types in terms of the overall costs of evacuation with high cost and low costs household types. Intuitively, compared to an average evacuation cost household type, high (low) cost household types should evacuate later (earlier) in general and also be less (more) willing to evacuate for higher (low) risk index storms. Figure 38 and 39 present the $\theta_{\left(T^{*}-n\right)}$ evacuation cut-off results for high cost and low cost household types respectively for all $\left(T^{*}-n\right)$ periods, $n=0,1, \ldots, 11$, along with the maximum risk index determined for each of these periods.

The high cost household optimal evacuation results from our multi-period model shown in Figure 38 coincide with the expected results for this household type. When the overall costs of evacuating increase by two times that of the average household, the evacuation region contracts for higher risk indices in periods $T^{*}$ to $\left(T^{*}-3\right)$. Furthermore, if the overall costs of evacuation have increased significantly, one would also expect the number of evacuees to decrease leading to lower rates of increase of evacuation costs for 
all periods compared to those used for an average household as given in Table 13 of Section V. When the higher costs of evacuation are coupled with evacuation cost increases half that of an average household, the evacuation region contracts even further.

Figure IV.38. High Overall Evacuation Cost Household Optimal Evacuation Results

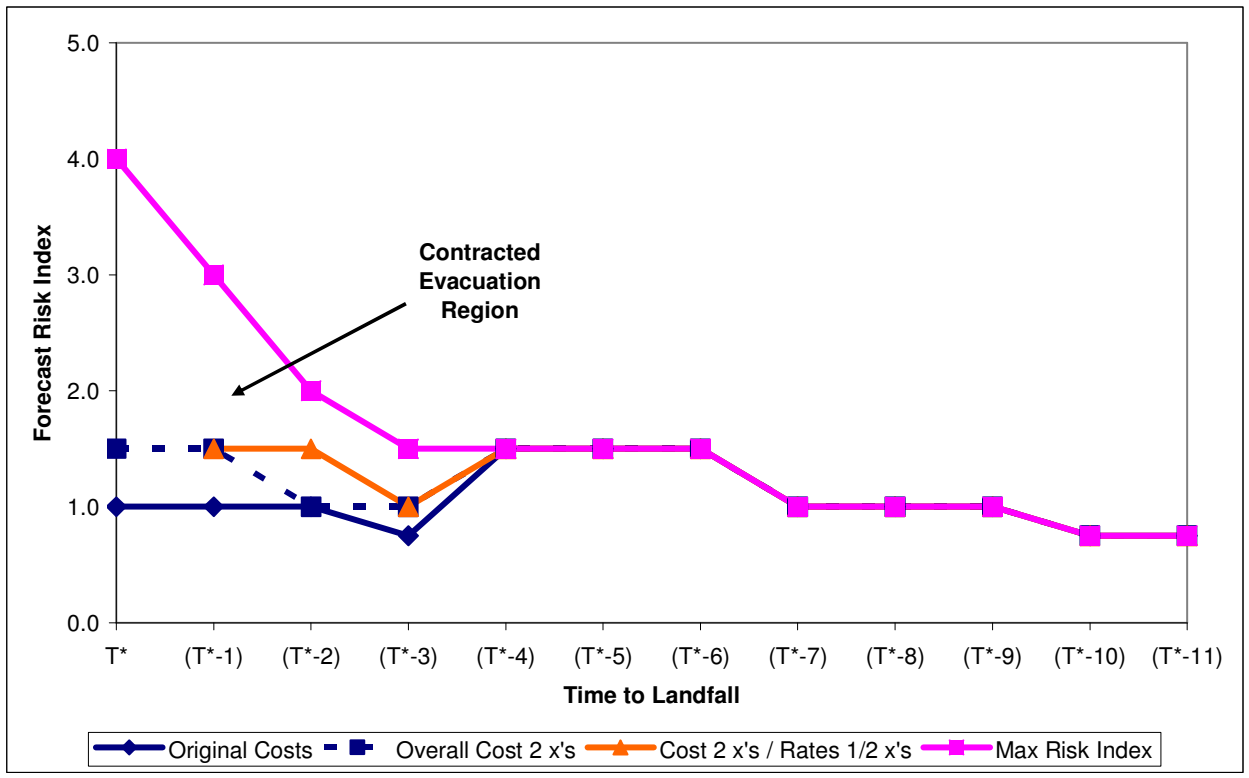

Likewise, the low cost household optimal evacuation results from our multiperiod model shown in Figure 39 coincide with the expected results for this household type. When the overall costs of evacuation have decreased to half of that of the average household, the evacuation region expands only slightly with evacuation optimal for lower risk indices in periods $T^{*}$ only. But if the overall costs of evacuation have decreased significantly, one would also expect the number of evacuees to increase leading to higher rates of increase of evacuation costs for all periods compared to those used for an average household as given in Table 13 of Section V. When the lower costs of evacuation are coupled with evacuation cost increases twice that of an average household, not only does 
the evacuation region expand for periods $T^{*}$ to $\left(T^{*}-3\right)$, but earlier evacuation is induced for periods $\left(T^{*_{-}} 5\right)$ to $\left(T^{*_{-}} 7\right)$.

Figure IV.39. Low Overall Evacuation Cost Household Optimal Evacuation Results

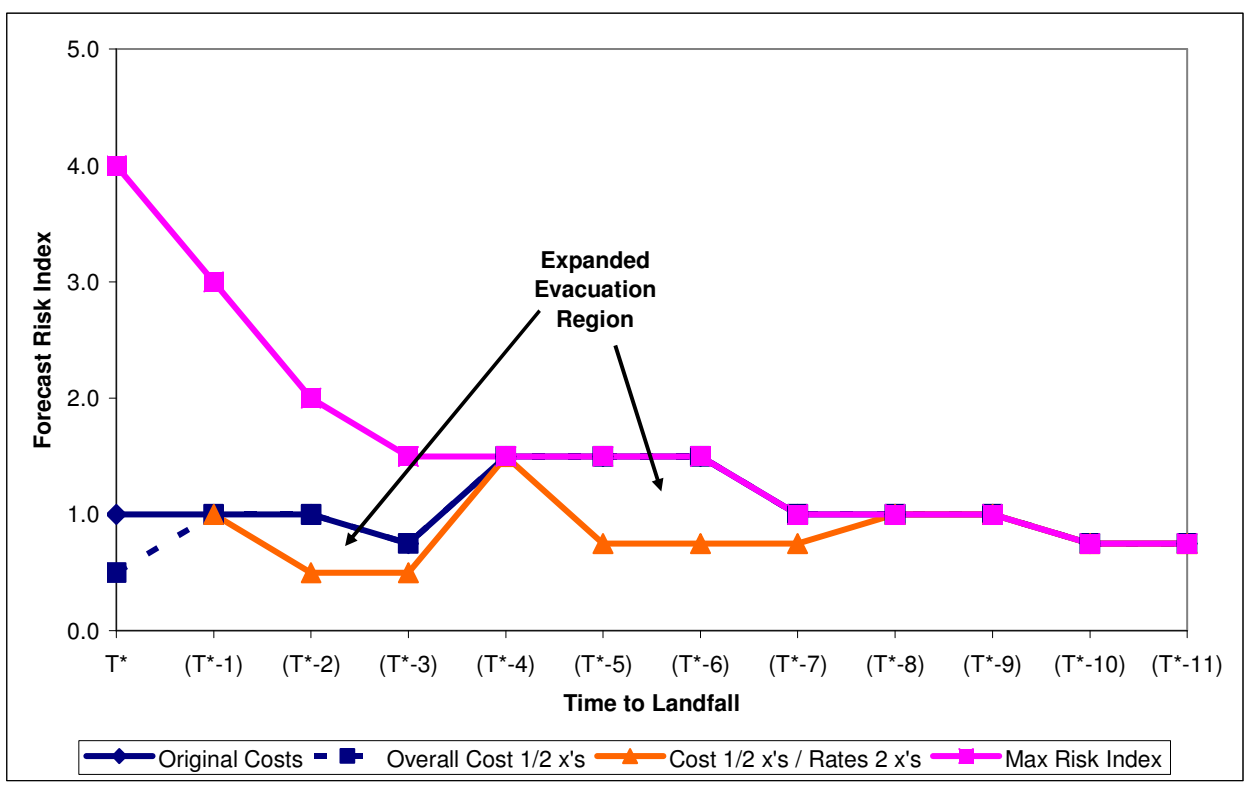

Finally, we can designate different evacuee types by changing the specific categories of our costs of evacuation. For example, evacuation cost structures for transients (tourists) can be constructed by increasing travel related and direct costs, and eliminating lost income. Figure 40 illustrates $\theta_{\left(T^{*}-n\right)}$ evacuation cut-off results for tourist types for all $\left(T^{\left.*_{-} n\right)}\right.$ periods, $n=0,1, \ldots, 11$, along with the maximum risk index determined for each of these periods. By increasing travel and direct costs by three times that of an average evacuee, as well as the rate of cost increases by twice that of an average evacuee, the evacuation region expand for periods $T^{*}$ to $\left(T^{*}-3\right)$, and earlier evacuation is induced for periods $\left(T^{*}-4\right)$ to $\left(T^{*}-7\right)$ as would be expected. These predicted earlier tourist evacuation results coincide with the faster rates of response for transients as illustrated in Figure 9 of Section II. 
Figure IV.40. Tourist Optimal Evacuation Results

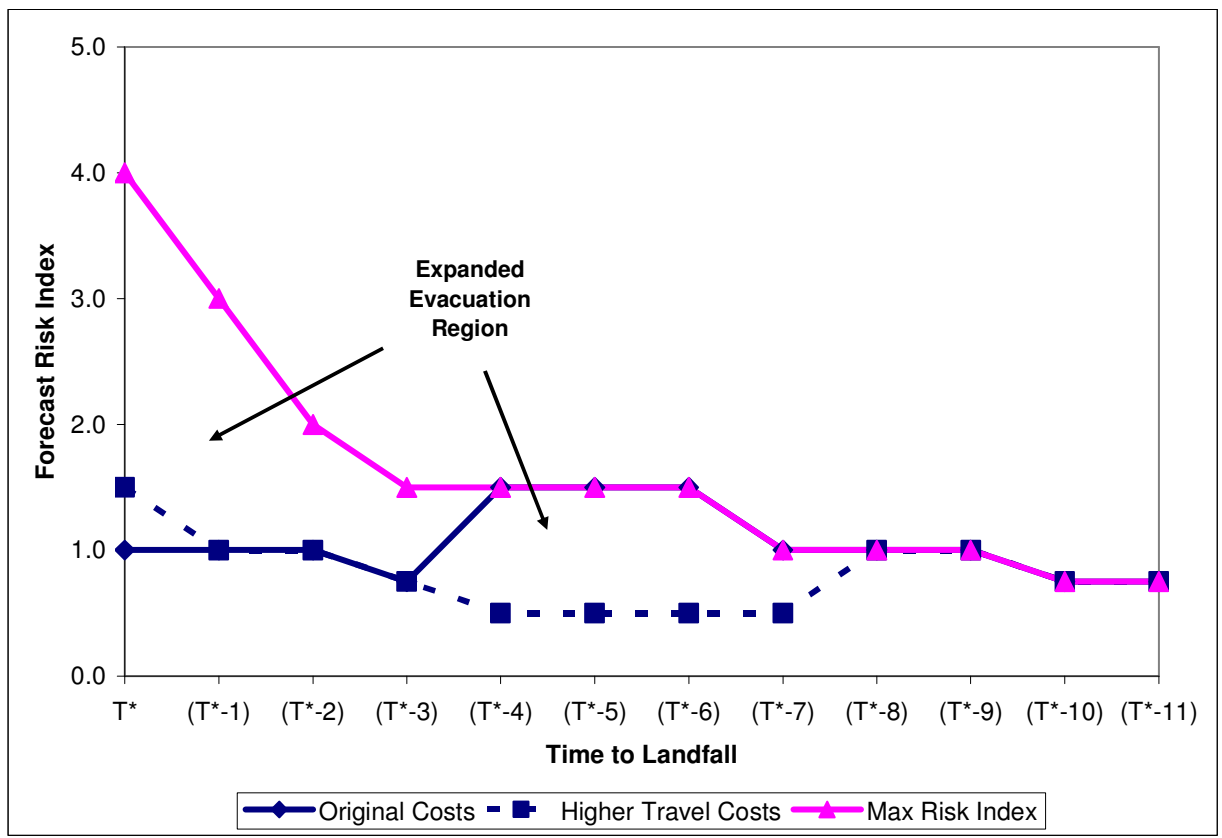

The results from our multi-period model by different household evacuation types also do a convincingly good job of predicting expected evacuation timing outcomes. Furthermore, these predicted results have the potential to explain the $10-50 \%$ of cumulative evacuations occurring between periods $\left(T^{*}-8\right)$ and $\left(T^{*}-4\right)$ for Hurricanes Lili and Ivan that our average household results from our general multi-period model could not. Given the illustrated precision of our model outcomes in regard to actual evacuation timing and expected evacuation response by various household types, we feel comfortable in further using the model to assess potential hurricane related policies meant to affect evacuation timing.

\section{IV.VII. Policy Implications}

In this section we provide a preliminary assessment of a number of potential hurricane policies meant to affect the timing of evacuation. 


\section{A. Evacuation Cost Reduction}

For policy makers and emergency managers interested in having households evacuate as early as possible, the costs of evacuation are a key constraint. Given that the costs of evacuation consist of the various components of direct, travel related, and lost income costs, a variety of policy initiatives may be available to reduce costs.

Figure 41 illustrates the effect of reducing the overall costs of evacuation equally across all components by $25 \%, 50 \%$, and $80 \%$, while holding all other variables constant. The results indicate that large cost reductions are needed, as much as $80 \%$ of the original, in order to induce evacuation for lower risk indices. Even these significant cost reductions do not induce earlier evacuation for periods prior to $\left(T^{*}-3\right)$. This suggests that a policy aimed at simply reducing the overall costs of evacuation does not induce early evacuation. These results indicate that a more targeted evacuation cost reduction, or a non-evacuation cost policy such as a focus on an improved forecast may be a more appropriate strategy in order to achieve earlier evacuations by the average household.

Figure IV.41. Effect of Lower Overall Costs of Evacuation on Evacuation Timing

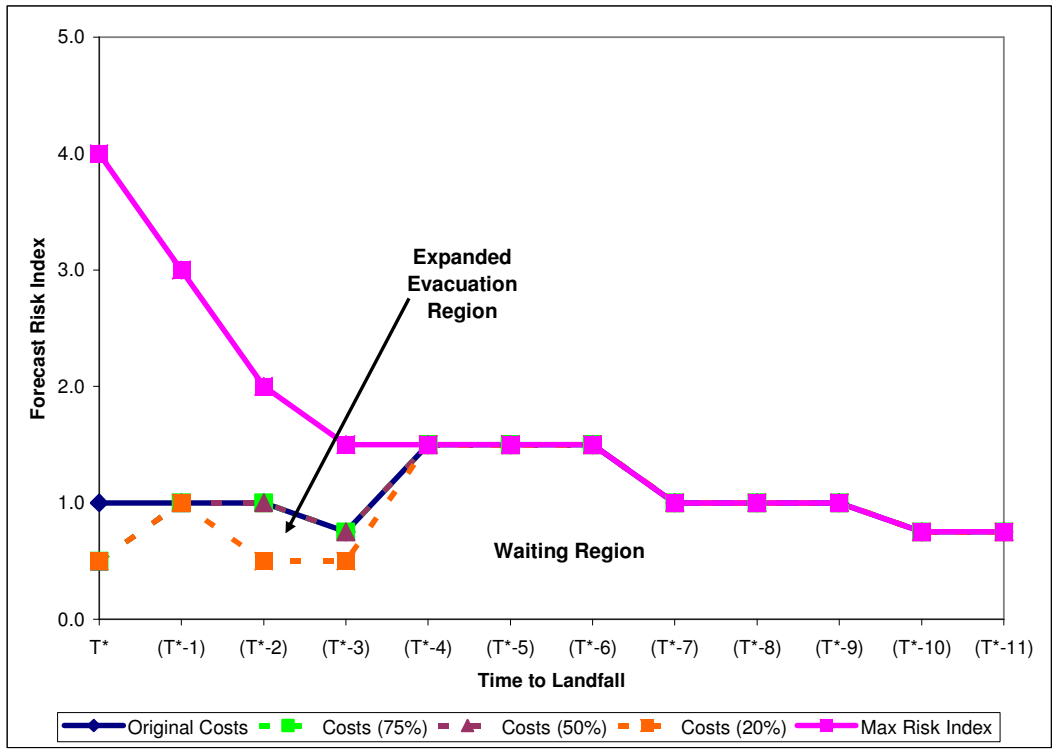




\section{B. The Value of an Improved Forecast}

In fact, we can use overall cost of evacuation reductions to estimate the value of an improved forecast in terms of its ability to induce earlier evacuations. As presented in Section VI, the evacuation response for Hurricane Opal was relatively late with the vast majority of evacuations occurring within 12 hours of the actual hurricane landfall. We repeat below for convenience the Hurricane Opal risk indices by location from Table 22 . From Table 22, there are two ways that an earlier evacuation 24 hours out from Opal's landfall in period $\left(T^{*}-3\right)$ could have occurred for the eventual landfall locations of Pensacola or Panama City: 1) lowering the $\left(T^{*}-3\right)$ cut-off point where evacuation is optimal from risk index values $>0.75$ to risk index values $>0.50$; or 2 ) increasing the $\left(T^{*}-3\right)$ risk index values achieved for Pensacola and Panama City to $>0.75$, the current cut-off point for optimal evacuation.

Table IV.22. Hurricane Opal Risk Indices by Location

\begin{tabular}{|c|c|c|c|c|c|c|c|c|c|c|c|c|}
\hline for: & $>1$ & $>1$ & $>1$ & $>.75$ & Wait & Wait & Wait & Wait & Wait & Wait & Wait & Wait \\
\hline Opal & O_T* & O_T*-1 & O_T*-2 & O_T*-3 & O_t -4 & O_t-5 & O_t-6 & O_t -7 & O_t-8 & O_t-9 & O_t-10 & O_t-11 \\
\hline 3.00 & 2.80 & 1.40 & 0.67 & 0.54 & 0.40 & 0.32 & 0.30 & 0.22 & 0.07 & 0.03 & 0.04 & 0.04 \\
\hline 0.00 & 1.84 & 1.08 & 0.70 & 0.56 & 0.36 & 0.28 & 0.26 & 0.22 & 0.05 & 0.02 & 0.04 & 0.03 \\
\hline 0.00 & 1.76 & 1.15 & 0.58 & 0.46 & 0.38 & 0.32 & 0.30 & 0.24 & 0.07 & 0.04 & 0.04 & 0.04 \\
\hline 0.00 & 0.92 & 0.83 & 0.67 & 0.54 & 0.34 & 0.26 & 0.26 & 0.20 & 0.05 & 0.02 & 0.04 & 0.03 \\
\hline 0.00 & 0.60 & 0.86 & 0.49 & 0.39 & 0.38 & 0.34 & 0.30 & 0.24 & 0.09 & 0.04 & 0.05 & 0.05 \\
\hline 0.00 & 0.60 & 0.65 & 0.61 & 0.49 & 0.30 & 0.24 & 0.24 & 0.18 & 0.04 & 0.00 & 0.03 & 0.02 \\
\hline 0.00 & 0.28 & 0.76 & 0.52 & 0.41 & 0.42 & 0.38 & 0.34 & 0.28 & 0.12 & 0.06 & 0.07 & 0.06 \\
\hline 0.00 & 0.00 & 0.29 & 0.36 & 0.29 & 0.34 & 0.34 & 0.30 & 0.26 & 0.12 & 0.06 & 0.06 & 0.06 \\
\hline 0.00 & 0.00 & 0.22 & 0.46 & 0.37 & 0.24 & 0.20 & 0.20 & 0.16 & 0.04 & 0.00 & 0.03 & 0.02 \\
\hline 0.00 & 0.00 & 0.07 & 0.30 & 0.24 & 0.18 & 0.16 & 0.18 & 0.16 & 0.04 & 0.00 & 0.03 & 0.02 \\
\hline 0.00 & 0.00 & 0.00 & 0.18 & 0.15 & 0.22 & 0.30 & 0.24 & 0.24 & 0.12 & 0.07 & 0.06 & 0.06 \\
\hline 0.00 & 0.00 & 0.00 & 0.06 & 0.05 & 0.08 & 0.16 & 0.12 & 0.18 & 0.12 & 0.07 & 0.05 & 0.06 \\
\hline 0.00 & 0.00 & 0.00 & 0.00 & 0.00 & 0.04 & 0.12 & 0.08 & 0.16 & 0.14 & 0.09 & 0.06 & 0.07 \\
\hline 0.00 & 0.00 & 0.00 & 0.00 & 0.00 & 0.00 & 0.10 & 0.06 & 0.14 & 0.14 & 0.10 & 0.06 & 0.07 \\
\hline 0.00 & 0.00 & 0.00 & 0.00 & 0.00 & 0.00 & 0.04 & 0.00 & 0.10 & 0.14 & 0.10 & 0.06 & 0.07 \\
\hline
\end{tabular}


In regard to lowering the $\left(T^{*_{-}} 3\right)$ cut-off point to risk index values $>0.50$, again Figure 41 indicates that for evacuation to be optimal for risk index values $>0.50$, overall evacuation costs need to be reduced by $80 \%$, which is a cost of $\$ 307$ per household. At a cost of $\$ 307$ per household, and with approximately 50,000 households in these two locations, total cost reductions necessary to induce evacuation 24 hours out from landfall could therefore plausibly equal $\$ 15$ million.

Conversely, in regard to increasing the $\left(T^{*}-3\right)$ risk index values to $>0.75$, the NHC strike probabilities for Panama City and Pensacola in this period were $22 \%$ and $23 \%$ respectively. If these strike probabilities had been increased to $31 \%$, risk index values would have been high enough for it to be rational for household to evacuate during period $\left(T^{*}-3\right)$, i.e., $>0.75$. Therefore, in the case of Hurricane Opal the difference between the cost necessary to improve the strike probabilities from $22 \%$ to $31 \% 24$ hours before landfall, and the $\$ 15$ million cost of evacuation reduction is the value of an improved forecast that induces evacuation 24 hours out from landfall.

\section{Salaried vs. Wage Employees}

The costs of lost income are one component of evacuation costs that potentially can be targeted by policy makers. Not only are the costs of lost income the largest component of the specified average costs of evacuation which we assumed to be incurred at period $\left(T^{*-4}\right)$, but they also delineate two separate household types with someone in the household having to work - hourly vs. salaried worker household types. We assume that salaried workers have more flexibility in their decision to evacuate with any missed 
days of work not equating to lost income, while hourly workers have less flexibility in their evacuation decision assuming that they lose their income for any days missed.

The results from our multi-period model with the costs of lost income eliminated demonstrate a divergent salaried vs. hourly worker outcome as shown in Figure 42. When the costs of lost income no longer need to be considered in the evacuation decision, earlier evacuation 2 days out from landfall in periods $\left(T^{*} 4\right)$ to $\left(T^{*_{-}} 7\right)$ is shown to be optimal for certain risk indices where waiting had previously been optimal. The elimination of lost income costs from the evacuation decision makes it easier to evacuate earlier.

Given the demonstrated hourly vs. salaried worker evacuation timing divergence, one can think of other possible policies that might make evacuation decisions more equitable such as a focus on the reduction of direct costs. For example, assume a policy that focuses on reducing much of the direct costs of evacuation through the use of improved shelters that provide meals, showers, etc. Households that use the improved shelters (which we assume to typically be hourly worker household types) have the possibility of having much of their direct costs eliminated. Figure 43 illustrates the evacuation region results when direct costs are completely eliminated from the multiperiod model where it is shown that little earlier evacuation is induced due to the elimination of direct costs.

The results from Figures 42 and 43 indicate an evacuation timing divergence between hourly and salaried workers. Furthermore, policies that give hourly workers more evacuation options once they have evacuated are not effective in minimizing the divergence. In order for the divergence to be addressed, policies need to be directed at 
making it easier for hourly workers to leave, such as a policy that provides incentives for employers to pay hourly workers for lost work time due to hurricane evacuations.

Figure IV.42. Optimal Evacuation Region Excluding Lost Income Costs

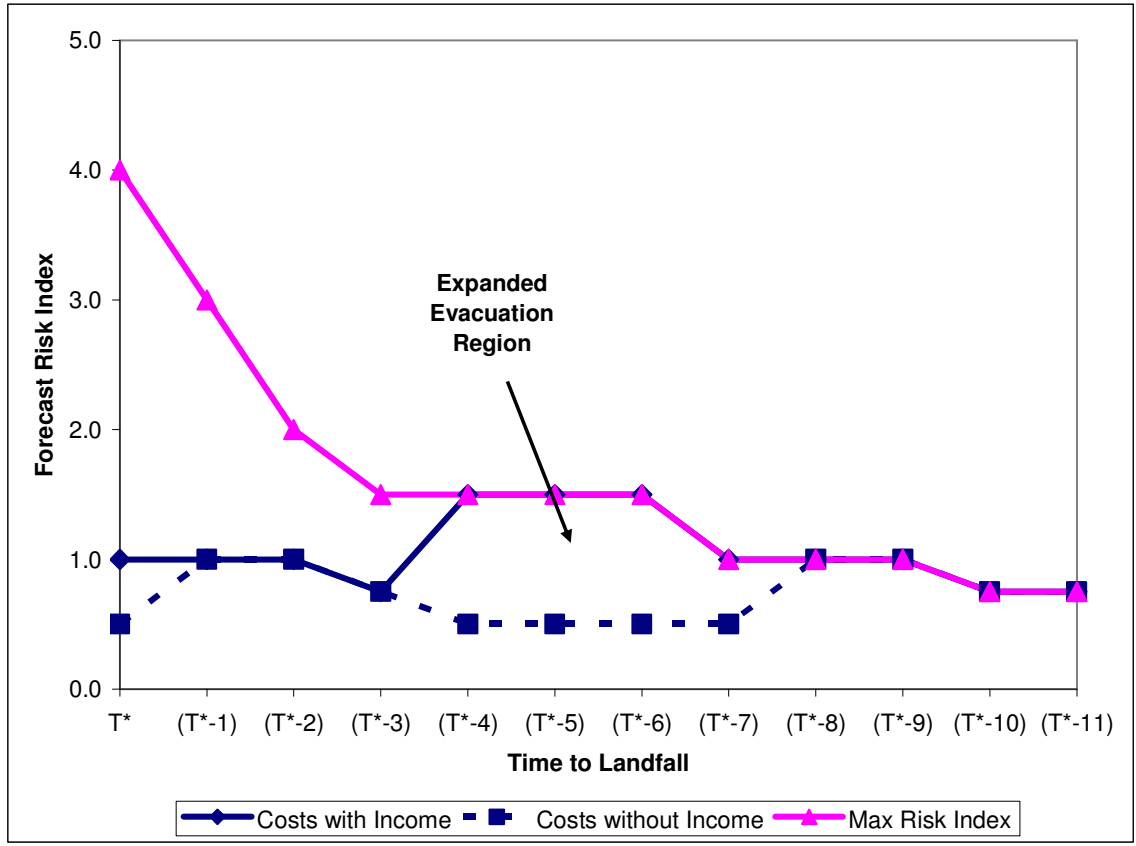

Figure IV.43. Optimal Evacuation Region Excluding Direct Costs

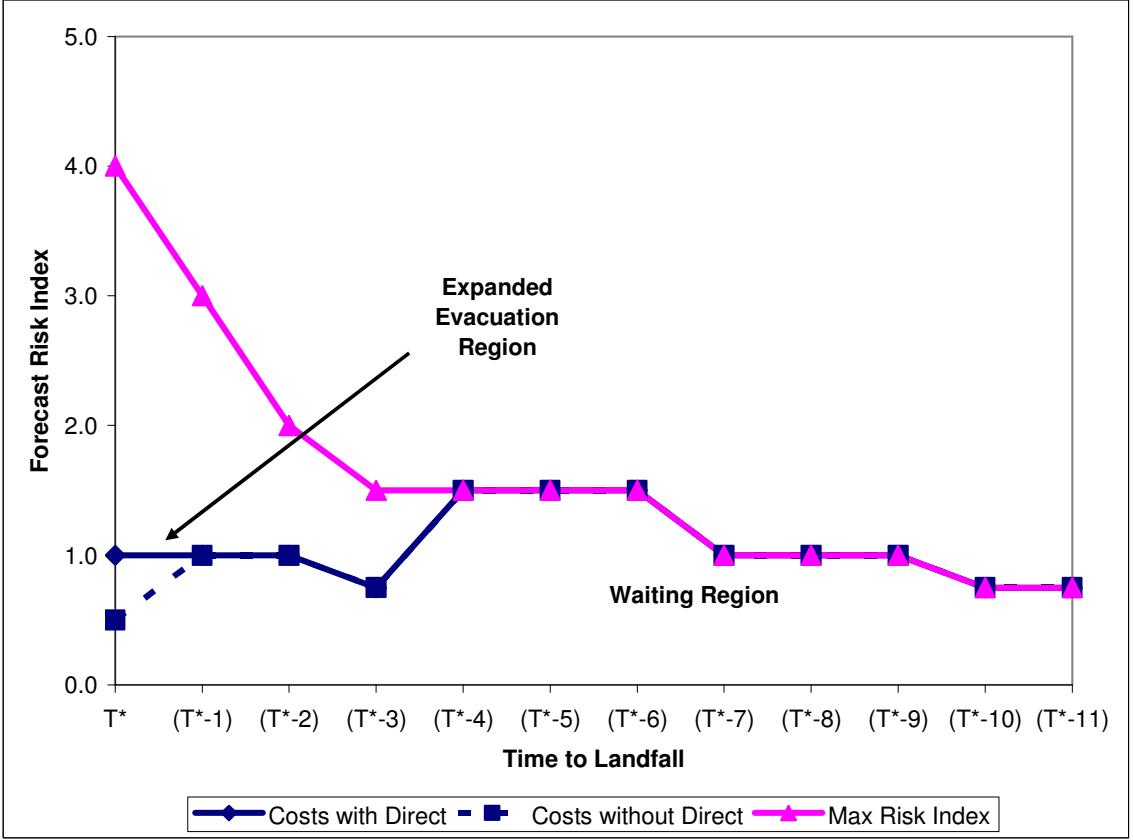




\section{The Cost Profile}

Other more targeted policies intended to induce earlier evacuation could focus on reducing the rates at which direct and travel-related costs increase over time such as the use of contra-flow or the increased availability of shelters. Figure 44 illustrates the affect on evacuation timing if these types of policies are implemented and our assumed rates of per period cost increases from $\left(T^{*}-11\right)$ to $T^{*}$ are decreased by half. Decreasing the rate at which the costs of evacuation increase over time leads to a contraction of the evacuation region, as well as to no earlier evacuation prior to period $\left(T^{*}-3\right)$ being induced. This outcome helps to explain the empirical result that Baker (2005b) found for Hurricane Ivan where when contra-flow was implemented, a quarter of respondents indicated this made them less likely to evacuate ${ }^{66}$. Similar to our results from the two-period model, these rate reduction results from the multi-period model show that when the ability to wait is a part of household's decision to evacuate, timing results may run opposite of the intended policy goals.

Conversely, when the rates of travel and direct costs increase over time, earlier evacuation is induced. Figure 44 also illustrates this result assuming the rates have increased by two times our original assumptions, with earlier evacuation shown for periods $\left(T^{*_{-} 5}\right)$ to $\left(T^{*_{-}} 7\right)$. This result also coincides with another Hurricane Ivan finding discussed by Baker (2005b) where the implementation of the contra-flow actually caused additional problems in traffic flow (which can be construed as a rate increase), and $60 \%$ of those evacuees that used the contra-flow route indicated that they would leave earlier next time.

\footnotetext{
${ }^{66}$ While a quarter of respondents said it made them less likely, $56 \%$ said it did make them more likely.
} 
Figure IV.44. Optimal Evacuation Region for Different Rates of Cost Increases

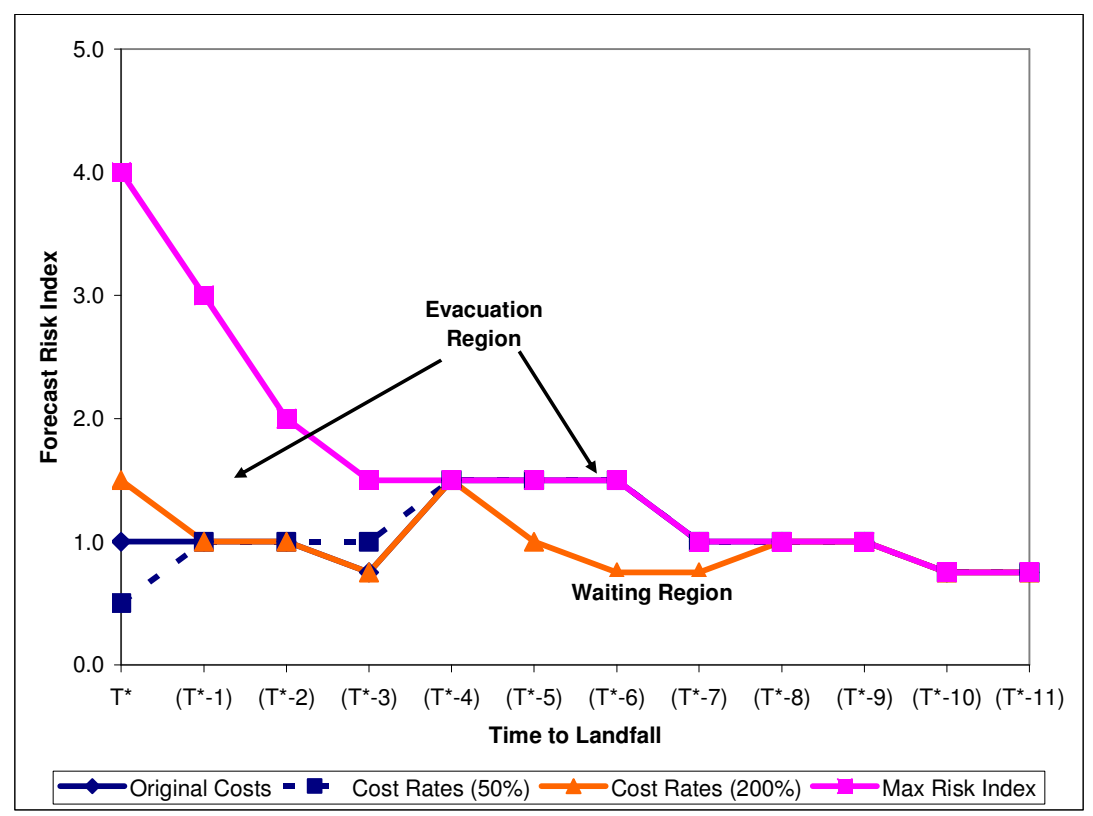

Finally, we can think of how different policies can be combined in order to induce earlier evacuation. Figure 37 of Section VI indicated that lowering the probability of being injured leads to a contracted evacuation region. In this way, a policy focused on structural mitigation would result in increased waiting by households. While this outcome may be desirable for inland homes, it would not be desirable for coastal homes. However, by combining a mitigation effort with cost reduction, the original evacuation cut-offs points can be achieved. Therefore, for coastal homes if mitigation efforts are introduced, they should be coupled with evacuation cost reduction policies. Also, from Figure 36 of Section VI we saw how conversely, higher probabilities for being injured lead to earlier evacuations. By introducing cost reductions of half the overall costs of evacuation in this situation, even earlier evacuation is induced as shown by Figure 45 . Cost reduction policies may therefore be more useful certain household types such as those located near the coast or in mobile homes. 
Figure IV.45. High Damage Household Combined with Cost Reduction Optimal Evacuation Results

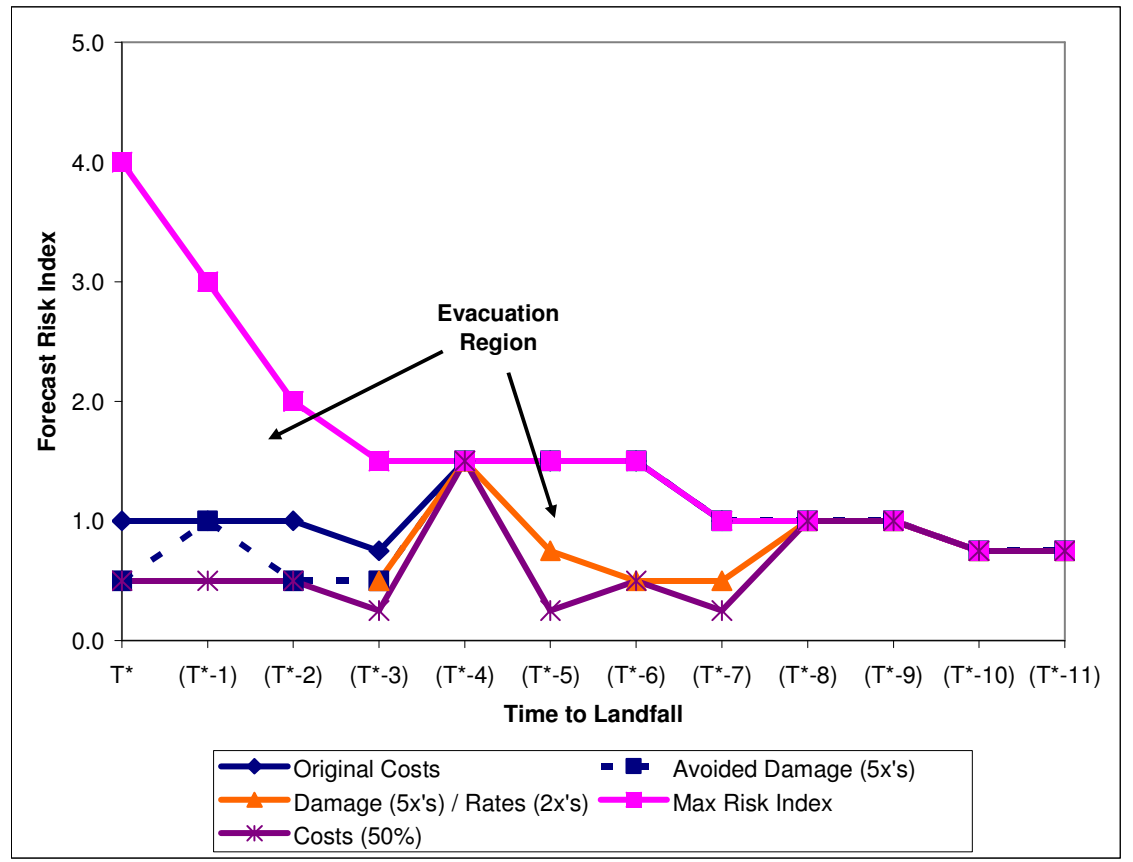

\section{IV.VIII. Conclusions}

This paper has developed of a dynamic model of hurricane evacuation behavior where every potential evacuation time period prior to the actual hurricane landfall, a household's optimal choice is to either evacuate, or to wait one more time period for a revised NHC hurricane forecast. The power and intuition behind framing a household's evacuation decision dynamically vs. statically was shown through a simple two-period model of evacuation. The dynamic model was extended to a realistic multi-period setup incorporating existing forecast and evacuation cost data in order to explain actual evacuation behavior for our designated Gulf of Mexico region. The evacuation timing results from our general model did a convincingly good job of explaining actual evacuation timing outcomes by location from specific hurricanes, as well as expected 
evacuation timing outcomes for various household types. Therefore, from our calibrated dynamic framework, a number of policy questions that plausibly affect the timing of household evacuations were analyzed. The dynamic model results were also able to provide a deeper understanding to existing evacuation timing empirical outcomes.

However, due to the probabilistic and non-primary nature of the model input data, we needed to develop a number of assumptions in order to properly incorporate the forecast and evacuation cost data into our multi-period dynamic evacuation model. While ultimately we feel comfortable in using the inputs as constructed from our assumptions and limited available data to draw initial results from our model, we acknowledge that the model and its inputs need to be further developed. Obtaining cost data that does not suffer from selection bias, generating more comprehensive forecast data and probability transition matrices through Monte Carlo simulations, incorporating the latest NHC forecast products such as wind speed probabilities; complementing the model with a formal econometric analysis of data collected through an actual evacuation survey; and a formal general equilibrium solution through specific cost functions all can be done to improve the existing model. Despite the current shortcomings, we feel the analysis has addressed the need for modeling hurricane evacuation behavioral responses in more precise and comprehensive ways, and importantly laid a solid foundation for continued development in this regard. 


\section{LIST OF REFERENCES}

Aadland, D., Caplan, A., Phillips, O., 2005. "A Bayesian Examination of Anchoring Bias and Cheap Talk in Contingent Valuation Studies", Working Paper

Adda, J., Cooper, R., 2003. Dynamic Economics. Cambridge, MA. The MIT Press.

Alberini, A., Kanninen, B., Carson, R., 1997. "Modeling Response Incentive Effects in Dichotomous Choice Contingent Valuation Data", Land Economics, 73:3:309324.

Baker, J., 2002. "Social Impacts of Tropical Cyclone Forecasts and Warnings", World Organization Bulletin. 229-235.

Baker, J., 2005a, Hurricane Charley Behavioral Analysis

Baker, J., 2005b, Hurricane Ivan Behavioral Analysis

Blake, E., Rappaport, E., Jarrell, J., Landsea, C., 2005. “The Deadliest, Costliest, And Most Intense United States Tropical Cyclones From 1851 To 2004 (And Other Frequently Requested Hurricane Facts)", NOAA Technical Memorandum NWS TPC-4

Boyle, K., Poor, J., Taylor, L., 1999. "Estimating the Demand for Protecting Freshwater Lakes from Eutrophication", American Journal of Agricultural Economics, 81:5:1118-1122.

Bricker, S., Clement, C., Pirhalla, D., Orlando, S., Farrow, D., 1999. National estuarine eutrophication assessment: effects of nutrient enrichment in the Nation's estuaries. NOAA, National Ocean Service, Special Projects Office, and the National Centers for Coastal Ocean Science, Silver Spring MD.

Cameron, T., Englin, J., 1997. "Respondent Experience and Contingent Valuation of Environmental Goods", Journal of Environmental Economics and Management, 33:296-313.

Cameron, T., James, M., 1987. "Efficient Estimation Methods for Closed-Ended Contingent Valuation Surveys", Review of Economics and Statistics, 69:2:269276.

Carson, R., Mitchell, R., Hanemann, W., Kopp, R., Presser, S., Ruud, P., 1992. A Contingent Valuation Study of Lost Passive Use Values Resulting from the Exxon Valdez Oil Spill. Report to the Attorney General of the State of Alaska 
Carson, R., Grove, T., Machina, M., 2000. "Incentive and Informational Properties of Preference Questions". University of California, San Diego Working Paper

Carson, R., Mitchell, R., Hanemann, W., Kopp, R., Presser, S., Ruud, P., 2003.

"Contingent Valuation and Lost Passive Use: Damages from the Exxon Valdez Oil Spill”, Environmental and Resource Economics, 25:257-286.

Chien, Y., Huang, C., Shaw, D., 2004. "A General Model of Starting Point Bias in Double-Bounded Dichotomous Contingent Valuation Surveys", Journal of Environmental Economics and Management, Article in Press.

Dash, N., Gladwin, H., 2005. "Evacuation Decision Making and Behavioral Responses: Individual and Household. Working Paper

Dixit, A., Pindyck, R., 1994. Investment Under Uncertainty. Princeton, NJ. Princeton University Press.

Dow, K., Cutter, S., 2002. "Emerging Hurricane Evacuation Issues: Hurricane Floyd and South Carolina", Natural Hazards Review, 3:6:12-18.

Epp, D., Al-Ani, K.S., 1979. "The Effect of Water Quality on Rural Nonfarm Residential Property Values”, American Journal of Agricultural Economics, 61:529-534.

FGDL (Florida Geographic Data Library), Retrieved November 2004 from http://www.fgdl.org/

Flachaire, E., Hollard, G., 2006. "Controlling Starting-Point Bias in Double-Bounded Contingent Valuation Surveys”, Land Economics, 82:1:103-111.

Flores, N., Strong, A., 2004. "Stated Preference Analysis of Public Goods: Are We Asking the Right Question?”, Working Paper.

FOS (Florida Oceanographic Society) St. Lucie River Estuary Water Quality Data, Retrieved October 2004 to January 2005 from http://www.floridaoceanographic.org/water.htm

Freeman, A.M. 2003. The Measurement of Environmental and Resource Values, $2^{\text {nd }}$ Edition Washington D.C., Resources For the Future

Frieser, B., 2004. Probabilistic Evacuation Decision Model for River Floods in the Netherlands Final Report

Fu, H., Wilmot, C., 2004. "A Sequential Logit Dynamic Travel Demand Model for Hurricane Evacuation" Working Paper 
Gibbs, J., Halstead, J., Boyle, K., Huang, J., 2002. “An Hedonic Analysis of the Effects of Lake Water Clarity on New Hampshire Lakefront Properties", Agricultural and Resource Economics Review, 31:1:39-46.

Gladwin, H., Lazo, J., Morrow, B., Peacock, W., Willoughby, H., 2005. "Social Science Research Needs for the Hurricane Forecast and Warning System", Working Paper.

Haab, T., McConnell, 2002. Valuing Environmental and Natural Resources: the Econometrics of non-market evaluation, Northampton, MA, Edward Elgar.

Hanemann, W., Loomis, J., Kanninen, B., 1991. "Statistical Efficiency of DoubleBounded Dichotomous Choice Contingent Valuation", American Journal of Agricultural Economics, 73:1255-1263.

Herriges, J., Shogren, J., 1996. "Starting Point Bias in Dichotomous Choice Valuation with Follow-Up Questioning", Journal of Environmental Economics and Management, 30:112-131.

Horowitz, J., 1993. “A New Model of Contingent Valuation”, American Journal of Agricultural Economics, 75:1268-1272.

HSH Associates, Retrieved November 2004 from www.hsh.com

HURREVAC, 2003. HURREVAC Training Manual.

Kristensen, A., 1996. Textbook Notes of Herd Management: Dynamic Programming and Markov Decision Processes

Leggett, C., Bockstael, N., 2000. "Evidence of the Effects of Water Quality on Residential Land Prices." Journal of Environmental Economics and Management, 39:121-144.

Letson, D., Sutter, D., Lazo, J., 2005. "The Economic Value Of Hurricane Forecasts: An Overview And Research Needs", Working paper.

Li, C., Mattson, L., 1995. "Discrete Choice Under Preference Uncertainty: An Improved Structural Model for Contingent Valuation", Journal of Environmental Economics and Management, 28:256-269.

Lindell, M., Prater, C., Wu, J., 2002. "Hurricane Evacuation Time Estimates for the Texas Gulf Coast", College Station, TX, HRRC, Texas A\&M University

Lindell, M.K. \& Perry, R.W. 2004. Communicating Environmental Risk in Multiethnic Communities. Thousand Oaks CA: Sage. 
Lindell, M., Prater, C., 2005. "Estimating Evacuation Time Components: Lessons from Nuclear Power Plants, Hurricanes, and the First World Trade Center Bombing", Workshop on Building Occupant Movement During Fire Emergencies. Proceedings. Session 4.5. June 10-11, 2004, Gaithersburg, MD, Peacock, R. D.; Kuligowski, E. D., Editor(s)(s), 91-95.

Lindell, M., Prater, C., Peacock, W., 2005a. "Organizational Communication and Decision Making in Hurricane Emergencies", Working paper.

Lindell, M., Lu, J., Prater, C., 2005b. "Household Decision Making and Evacuation in Response to Hurricane Lili", Natural Hazards Review, 6:4:171-179.

Loomis, J., Ekstrand, E., 1998. "Alternative approaches for incorporating respondent uncertainty when estimating willingness to pay: the case of the Mexican spotted owl”, Ecological Economics, 27:29-41.

Park, J., 2003. "A Test of the Answering Mechanisms of the Double-Bounded Contingent Valuation Method", Applied Economics Letters, 10:975-984.

McLeod, D., Bergland, O., 1999. "Willingness-To-Pay Estimates Using the DoubleBounded Dichotomous-Choice Contingent Valuation Format: A Test for Validity and Precision in a Bayesian Framework", Land Economics, 75:1:115-125.

MCPA (Martin County Property Appraiser), Retrieved October to November 2004 from http://paoweb.martin.fl.us/

Michael, H., Boyle, K., Bouchard, R., 2000. "Does the Measurement of Environmental Quality Affect Implicit Prices Estimated from Hedonic Models?", Land Economics, 76:2:283-298.

MMC (Multihazard Mitigation Council), 2005. Natural Hazard Mitigation Saves: An Independent Study to Assess the Future Savings from Mitigation Activities. National Institute of Building Sciences, Washington D.C.

National Hurricane Center (NHC) http://www.nhc.noaa.gov/index.shtml

Norcross, B., 2006. Hurricane Almanac 2006. New York, NY. St. Martin's Griffin.

OFHEO (Office of Federal Housing Enterprise Oversight), Retrieved November 2004 from http://www.ofheo.gov/index.asp

Olson, L., 1990. "The Search for a Safe Environment: The Economics of Screening and Regulating Environmental Hazards", Journal of Environmental Economics and Management, 19:1-18. 
Poor, P., Boyle, K., Taylor, L., Bouchard, R., 2001. "Objective versus Subjective Measures of Water Clarity in Hedonic Property Value Models", Land Economics, $77: 4: 482-493$.

Ready, R., Whitehead, J., Blomquist, G., 1995. "Contingent Valuation when Respondents are Ambivalent", Journal of Environmental Economics and Management, 29:181196.

Regnier, E., 2006. “Evacuation Decisions and Hurricane Track Probability”, Working Paper

Stevenson, S., 2004. "New Empirical Evidence on Heteroscedasticity in Hedonic Housing Models", Journal of Housing Economics, 13:136-153.

Taylor, L., 2003. The Hedonic Method. In P. Champ, K. Boyle, and T. Brown (Eds.), A Primer on Nonmarket Valuation (pgs. 331-393). Kluwer Academic Publishers.

USACE, 2006A.

http://chps.sam.usace.army.mil/USHESdata/Louisiana/SWLA/cd/html/chapters/chapter0 4/chapter_04_results.html

USACE, 2006B.

http://chps.sam.usace.army.mil/ushesdata/Post_Storm_Assessment_page.htm

USACE/SFWMD (United States Army Corps of Engineers, South Florida Water Management District), 2002. Central and Southern Florida Project Indian River Lagoon - South (IRLS) Feasibility Study, Final Integrated Feasibility Report and Supplemental Environmental Impact Statement.

Wang, H., 1997. "Treatment of “'Don't-Know”' Responses in Contingent Valuation Surveys: A Random Valuation Model", Journal of Environmental Economics and Management, 32:219-232.

Whitehead, J., 2002. "Incentive Incompatibility and Starting-Point Bias in Iterative Valuation Questions", Land Economics, 78:2:285-297

Whitehead, J., 2003. "One Million Dollars Per Mile? The Opportunity Cost Of Hurricane Evacuation”, Ocean and Coastal Management, 46:1069-1083. 


\section{APPENDIX}

Derived Hurricane Risk Indices Per Storm, Per Location, \& Per Period

\begin{tabular}{|c|c|c|c|c|c|c|c|c|c|c|c|c|c|c|c|}
\hline Arlene (20 & 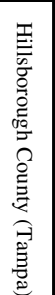 & 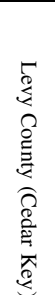 & 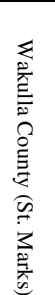 & 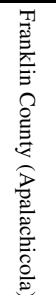 & 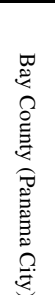 & 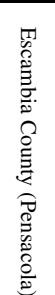 & 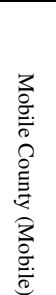 & 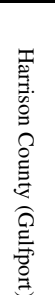 & 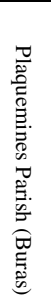 & 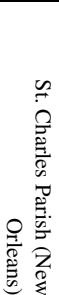 & 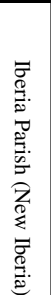 & 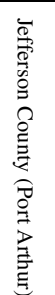 & 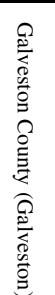 & 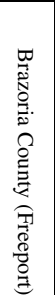 & 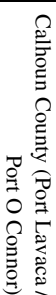 \\
\hline T* & 0.00 & 0.00 & 0.00 & 0.00 & 0.00 & 0.00 & 0.00 & 0.00 & 0.00 & 0.00 & 0.00 & 0.00 & 0.00 & 0.00 & 0.00 \\
\hline$\left(T^{*}-1\right)$ & 0.00 & 0.00 & 0.01 & 0.05 & 0.13 & 0.33 & 0.34 & 0.30 & 0.22 & 0.14 & 0.01 & 0.00 & 0.00 & 0.00 & 0.00 \\
\hline$(\mathrm{T} *-2)$ & 0.00 & 0.00 & 0.05 & 0.11 & 0.17 & 0.24 & 0.23 & 0.20 & 0.15 & 0.11 & 0.02 & 0.00 & 0.00 & \begin{tabular}{|l|}
0.00 \\
\end{tabular} & 0.00 \\
\hline$(\mathrm{T} *-3)$ & 0.00 & 0.01 & 0.04 & 0.11 & 0.13 & 0.14 & 0.12 & 0.10 & 0.08 & 0.06 & 0.02 & 0.00 & 0.00 & 0.00 & 0.00 \\
\hline$(T *-4)$ & 0.00 & 0.01 & 0.03 & 0.06 & 0.07 & 0.10 & 0.11 & 0.10 & 0.09 & 0.07 & 0.03 & 0.01 & 0.00 & 0.00 & 0.00 \\
\hline$\left(T^{*}-5\right)$ & 0.00 & 0.01 & 0.02 & 0.02 & 0.03 & 0.04 & 0.05 & 0.05 & 0.05 & 0.05 & 0.03 & 0.01 & 0.01 & \begin{tabular}{|l|}
0.00 \\
\end{tabular} & 0.00 \\
\hline$\left(T^{*}-6\right)$ & 0.00 & 0.01 & 0.02 & 0.02 & 0.03 & 0.04 & 0.05 & 0.05 & 0.05 & 0.05 & 0.03 & 0.01 & 0.01 & 0.00 & 0.00 \\
\hline$(T *-7)$ & 0.00 & 0.00 & 0.00 & 0.00 & 0.01 & 0.01 & 0.01 & 0.01 & 0.01 & 0.01 & 0.01 & 0.00 & 0.00 & 0.00 & 0.00 \\
\hline$\left(T^{*}-8\right)$ & 0.00 & 0.00 & 0.00 & 0.01 & 0.01 & 0.01 & 0.01 & 0.01 & 0.01 & 0.01 & 0.00 & 0.00 & 0.00 & 0.00 & 0.00 \\
\hline$(T *-9)$ & 0.00 & 0.00 & 0.00 & 0.01 & 0.01 & 0.01 & 0.01 & 0.01 & 0.01 & 0.01 & 0.00 & 0.00 & 0.00 & 0.00 & 0.00 \\
\hline$(\mathrm{T} *-10)$ & 0.00 & 0.00 & 0.00 & 0.00 & 0.00 & 0.00 & 0.00 & 0.00 & 0.01 & 0.00 & 0.00 & 0.00 & 0.00 & 0.00 & 0.00 \\
\hline$\left(\mathrm{T}^{*}-11\right)$ & 0.01 & 0.01 & 0.01 & 0.01 & 0.01 & 0.01 & 0.01 & 0.02 & 0.02 & 0.02 & 0.01 & 0.01 & 0.01 & \begin{tabular}{|l|}
0.01 \\
\end{tabular} & 0.00 \\
\hline
\end{tabular}

\begin{tabular}{|c|c|c|c|c|c|c|c|c|c|c|c|c|c|c|c|}
\hline Cindy (2005) & 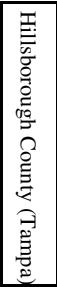 & 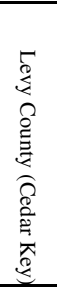 & 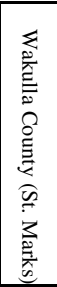 & 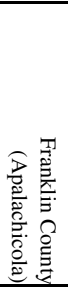 & 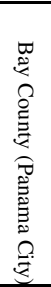 & 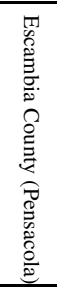 & 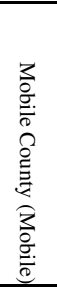 & 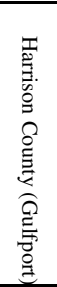 & 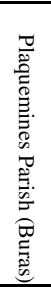 & 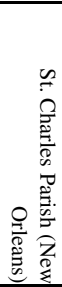 & 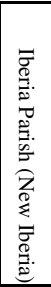 & 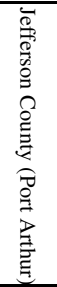 & 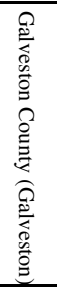 & 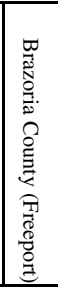 & $\begin{array}{r} \\
\\
0 \\
0 \\
0 \\
0 \\
0 \\
0 \\
0 \\
0 \\
0 \\
0 \\
0 \\
0 \\
0 \\
0 \\
0 \\
0 \\
0 \\
0\end{array}$ \\
\hline $\mathrm{T}^{*}$ & 0.00 & 0.00 & 0.00 & \begin{tabular}{|l|l|}
0.00 \\
\end{tabular} & 0.00 & 0.00 & 0.00 & 0.00 & 0.00 & 0.00 & 0.00 & 0.00 & 0.00 & 0.00 & 0.00 \\
\hline$\left(\mathrm{T}^{*}-1\right)$ & 0.00 & 0.00 & 0.00 & 0.00 & 0.00 & 0.00 & 0.00 & 0.00 & 0.00 & 0.00 & 0.00 & 0.00 & 0.00 & 0.00 & 0.00 \\
\hline$\left(\mathrm{T}^{*}-2\right)$ & \begin{tabular}{|l|l|}
0.00 \\
\end{tabular} & 0.00 & 0.00 & 0.00 & 0.00 & 0.00 & 0.00 & 0.00 & 0.00 & 0.00 & 0.00 & 0.00 & 0.00 & 0.00 & 0.00 \\
\hline$\left(\mathrm{T}^{*}-3\right)$ & \begin{tabular}{|l|l|}
0.00 \\
\end{tabular} & 0.00 & 0.00 & 0.00 & 0.00 & 0.00 & 0.00 & 0.00 & 0.00 & 0.00 & 0.00 & 0.00 & 0.00 & 0.00 & 0.00 \\
\hline$\left(\mathrm{T}^{*}-4\right)$ & \begin{tabular}{|l|l|}
0.00 \\
\end{tabular} & 0.00 & 0.00 & 0.00 & 0.00 & 0.00 & 0.00 & 0.00 & 0.00 & 0.00 & 0.00 & 0.00 & 0.00 & 0.00 & 0.00 \\
\hline$\left(\mathrm{T}^{*}-5\right)$ & 0.00 & 0.00 & 0.00 & 0.00 & 0.00 & 0.01 & 0.01 & 0.02 & 0.02 & 0.02 & 0.03 & 0.03 & 0.04 & 0.04 & 0.03 \\
\hline$\left(\mathrm{T}^{*}-6\right)$ & \begin{tabular}{|l|l|}
0.00 \\
\end{tabular} & 0.00 & \begin{tabular}{|l|}
0.00 \\
\end{tabular} & 0.00 & 0.01 & 0.01 & 0.01 & \begin{tabular}{|l|l}
0.02 \\
\end{tabular} & 0.02 & 0.03 & 0.03 & 0.04 & 0.04 & 0.04 & \begin{tabular}{|l|}
0.04 \\
\end{tabular} \\
\hline$\left(\mathrm{T}^{*}-7\right)$ & \begin{tabular}{|l|}
0.00 \\
\end{tabular} & 0.00 & 0.00 & \begin{tabular}{|l|}
0.00 \\
\end{tabular} & 0.00 & 0.01 & 0.01 & \begin{tabular}{|l|}
0.01 \\
\end{tabular} & 0.02 & 0.02 & 0.03 & 0.03 & 0.04 & 0.04 & \begin{tabular}{|l|l|}
0.04 \\
\end{tabular} \\
\hline$\left(\mathrm{T}^{*}-8\right)$ & \begin{tabular}{|l|}
0.00 \\
\end{tabular} & 0.00 & \begin{tabular}{|l|l|}
0.00 \\
\end{tabular} & 0.00 & 0.00 & 0.00 & 0.00 & \begin{tabular}{|l|}
0.00 \\
\end{tabular} & 0.00 & 0.00 & 0.00 & 0.00 & 0.00 & 0.00 & \begin{tabular}{|l|}
0.00 \\
\end{tabular} \\
\hline$\left(\mathrm{T}^{*}-9\right)$ & & & & & & & & & & & & & & & \\
\hline$\left(T^{*}-10\right)$ & & & & & & & & & & & & & & & \\
\hline$(\mathrm{T} *-11)$ & & & & & & & & & & & & & & & \\
\hline
\end{tabular}

\begin{tabular}{|c|c|c|c|c|c|c|c|c|c|c|c|c|c|c|c|}
\hline Dennis (2005) & 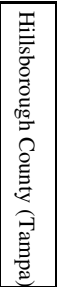 & 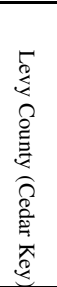 & 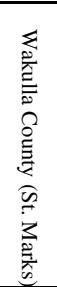 & 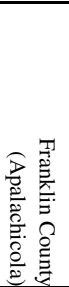 & 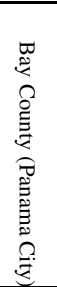 & 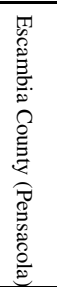 & 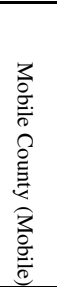 & 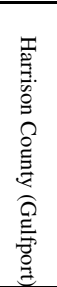 & 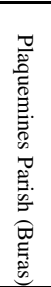 & 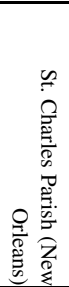 & 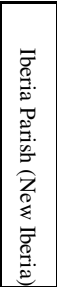 & 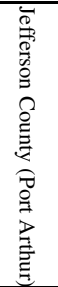 & 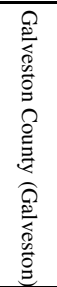 & 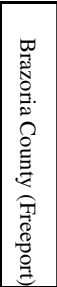 & 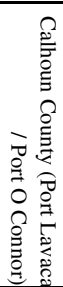 \\
\hline $\mathrm{T}^{*}$ & 0.00 & 0.00 & \begin{tabular}{|l|}
0.00 \\
\end{tabular} & 0.12 & 1.12 & 3.76 & 2.92 & 0.48 & 0.00 & 0.08 & \begin{tabular}{|l|}
0.00 \\
\end{tabular} & 0.00 & 0.00 & \begin{tabular}{|l|}
0.00 \\
\end{tabular} & 0.00 \\
\hline$\left(\mathrm{T}^{*}-1\right)$ & 0.00 & 0.00 & 0.12 & 0.60 & 1.08 & 2.00 & 2.00 & 1.68 & 0.72 & 0.68 & \begin{tabular}{|l|}
0.12 \\
\end{tabular} & 0.00 & 0.00 & \begin{tabular}{|l|}
0.00 \\
\end{tabular} & 0.00 \\
\hline$\left(\mathrm{T}^{*}-2\right)$ & 0.00 & 0.00 & 0.32 & 0.76 & 1.03 & 1.51 & 1.47 & 1.31 & 0.95 & 0.80 & 0.32 & 0.08 & 0.00 & 0.00 & 0.00 \\
\hline$\left(\mathrm{T}^{*}-3\right)$ & 0.10 & 0.20 & \begin{tabular}{|l|}
0.46 \\
\end{tabular} & 0.83 & 0.96 & 1.16 & 1.09 & 0.93 & 0.60 & 0.50 & \begin{tabular}{|l|}
0.17 \\
\end{tabular} & 0.00 & 0.00 & \begin{tabular}{|l|}
0.00 \\
\end{tabular} & 0.00 \\
\hline$\left(\mathrm{T}^{*}-4\right)$ & \begin{tabular}{|l|}
0.17 \\
\end{tabular} & 0.32 & 0.59 & 0.90 & 0.93 & 0.88 & 0.73 & 0.59 & 0.39 & \begin{tabular}{|l|}
0.29 \\
\end{tabular} & 0.07 & 0.00 & 0.00 & 0.00 & 0.00 \\
\hline$\left(\mathrm{T}^{*}-5\right)$ & $\begin{array}{ll}0.57 \\
\end{array}$ & 0.60 & 0.80 & 1.11 & 1.14 & 1.07 & 0.94 & 0.80 & 0.57 & 0.47 & \begin{tabular}{|l|}
0.17 \\
\end{tabular} & 0.00 & 0.00 & \begin{tabular}{|l|}
0.00 \\
\end{tabular} & 0.00 \\
\hline$\left(\mathrm{T}^{*}-6\right)$ & \begin{tabular}{|l|}
0.56 \\
\end{tabular} & 0.54 & \begin{tabular}{|l|}
0.59 \\
\end{tabular} & 0.73 & 0.73 & 0.67 & 0.59 & 0.51 & 0.40 & 0.35 & \begin{tabular}{|l|}
0.19 \\
\end{tabular} & 0.05 & 0.00 & \begin{tabular}{|l|}
0.00 \\
\end{tabular} & 0.00 \\
\hline$\left(\mathrm{T}^{*}-7\right)$ & 0.76 & 0.70 & \begin{tabular}{|l|}
0.73 \\
\end{tabular} & 0.86 & 0.83 & 0.73 & 0.66 & 0.56 & 0.46 & 0.43 & \begin{tabular}{|l|}
0.26 \\
\end{tabular} & 0.10 & 0.00 & \begin{tabular}{|l|}
0.00 \\
\end{tabular} & 0.00 \\
\hline$\left(\mathrm{T}^{*}-8\right)$ & \begin{tabular}{|l|}
0.71 \\
\end{tabular} & 0.64 & \begin{tabular}{|l|}
0.67 \\
\end{tabular} & 0.74 & 0.74 & 0.71 & 0.64 & 0.60 & 0.53 & 0.50 & \begin{tabular}{|l|}
0.32 \\
\end{tabular} & 0.14 & 0.07 & \begin{tabular}{|l|}
0.00 \\
\end{tabular} & 0.00 \\
\hline$\left(\mathrm{T}^{*}-9\right)$ & \begin{tabular}{|l|}
0.71 \\
\end{tabular} & 0.64 & \begin{tabular}{|l|}
0.64 \\
\end{tabular} & 0.71 & 0.71 & 0.64 & 0.60 & 0.57 & 0.53 & 0.46 & \begin{tabular}{|l|}
0.32 \\
\end{tabular} & 0.14 & 0.07 & \begin{tabular}{|l|}
0.00 \\
\end{tabular} & 0.00 \\
\hline$\left(\mathrm{T}^{*}-10\right)$ & \begin{tabular}{|l|}
0.57 \\
\end{tabular} & 0.51 & \begin{tabular}{|l|}
0.51 \\
\end{tabular} & 0.57 & 0.57 & 0.51 & 0.46 & 0.43 & 0.38 & \begin{tabular}{|l|}
0.32 \\
\end{tabular} & \begin{tabular}{|l|}
0.19 \\
\end{tabular} & 0.05 & 0.00 & \begin{tabular}{|l|}
0.00 \\
\end{tabular} & 0.00 \\
\hline$(\mathrm{T} *-11)$ & $\begin{array}{ll}0.60 \\
\end{array}$ & 0.57 & \begin{tabular}{|l|}
0.54 \\
\end{tabular} & 0.60 & 0.60 & 0.54 & 0.48 & 0.42 & 0.39 & 0.33 & \begin{tabular}{|l|}
0.18 \\
\end{tabular} & 0.00 & 0.00 & \begin{tabular}{|l|}
0.00 \\
\end{tabular} & 0.00 \\
\hline
\end{tabular}




\begin{tabular}{|c|c|c|c|c|c|c|c|c|c|c|c|c|c|c|c|}
\hline Katrina (2005) & 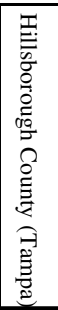 & 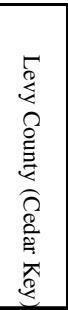 & 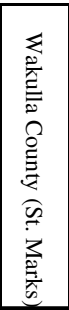 & 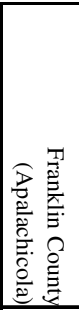 & 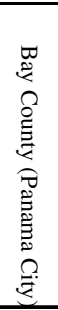 & 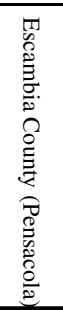 & 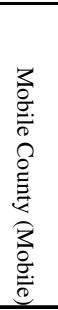 & 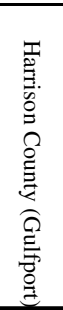 & 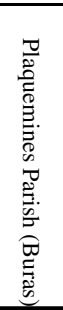 & 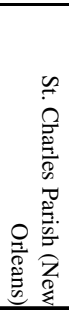 & 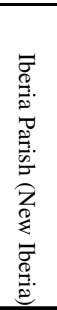 & 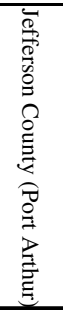 & 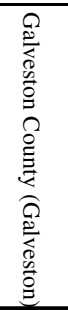 & 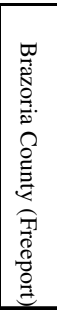 & 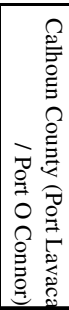 \\
\hline $\mathrm{T}^{*}$ & 0.00 & \begin{tabular}{|l|}
0.00 \\
\end{tabular} & \begin{tabular}{|l|}
0.00 \\
\end{tabular} & 0.00 & 0.10 & 1.10 & 2.00 & 2.70 & 3.85 & 2.95 & 1.45 & 0.00 & 0.00 & 0.00 & 0.00 \\
\hline$\left(T^{*}-1\right)$ & 0.00 & \begin{tabular}{|l|}
0.00 \\
\end{tabular} & \begin{tabular}{|l|}
0.10 \\
\end{tabular} & 0.10 & 0.25 & 0.90 & 1.50 & 1.90 & 2.60 & 2.35 & 1.60 & 0.25 & 0.00 & 0.00 & 0.00 \\
\hline$\left(\mathrm{T}^{*}-2\right)$ & 0.00 & \begin{tabular}{|l|}
0.00 \\
\end{tabular} & \begin{tabular}{|l|}
0.28 \\
\end{tabular} & 0.38 & 0.57 & 1.09 & 1.37 & 1.56 & 1.80 & 1.65 & 1.09 & 0.24 & 0.00 & 0.00 & 0.00 \\
\hline$\left(\mathrm{T}^{*}-3\right)$ & 0.00 & \begin{tabular}{|l|}
0.08 \\
\end{tabular} & \begin{tabular}{|l|}
0.28 \\
\end{tabular} & 0.37 & 0.45 & 0.77 & 0.94 & 1.06 & 1.22 & 1.18 & 0.98 & 0.49 & 0.24 & 0.08 & 0.00 \\
\hline$\left(T^{*}-4\right)$ & 0.00 & \begin{tabular}{|l|}
0.12 \\
\end{tabular} & \begin{tabular}{|l|}
0.28 \\
\end{tabular} & 0.37 & 0.49 & 0.69 & 0.81 & 0.94 & 1.10 & 1.06 & 0.94 & 0.57 & 0.41 & 0.28 & 0.12 \\
\hline$\left(T^{*}-5\right)$ & 0.08 & \begin{tabular}{|l|}
0.16 \\
\end{tabular} & \begin{tabular}{|l|}
0.32 \\
\end{tabular} & 0.41 & 0.49 & 0.65 & 0.73 & 0.81 & 0.93 & 0.85 & 0.81 & 0.61 & 0.49 & 0.41 & 0.24 \\
\hline$\left(T^{*}-6\right)$ & 0.12 & \begin{tabular}{|l|}
0.20 \\
\end{tabular} & \begin{tabular}{|l|}
0.37 \\
\end{tabular} & 0.45 & 0.49 & 0.61 & 0.69 & 0.73 & 0.85 & 0.77 & 0.73 & 0.57 & 0.49 & 0.41 & 0.28 \\
\hline$\left(T^{*}-7\right)$ & 0.15 & \begin{tabular}{|l|}
0.19 \\
\end{tabular} & \begin{tabular}{|l|}
0.30 \\
\end{tabular} & 0.37 & 0.41 & 0.48 & 0.52 & 0.59 & 0.67 & 0.63 & 0.59 & 0.48 & 0.45 & 0.41 & 0.30 \\
\hline$(\mathrm{T} *-8)$ & 0.11 & \begin{tabular}{|l|}
0.18 \\
\end{tabular} & \begin{tabular}{|l|}
0.32 \\
\end{tabular} & 0.46 & 0.53 & 0.60 & 0.63 & 0.63 & 0.67 & 0.60 & 0.49 & 0.32 & 0.25 & 0.18 & 0.11 \\
\hline$\left(T^{*}-9\right)$ & 0.39 & \begin{tabular}{|l|}
0.42 \\
\end{tabular} & \begin{tabular}{|l|}
0.46 \\
\end{tabular} & 0.60 & 0.60 & 0.60 & 0.56 & 0.56 & 0.60 & 0.53 & 0.42 & 0.25 & 0.18 & 0.14 & 0.07 \\
\hline$\left(\mathrm{T}^{*}-10\right)$ & 0.62 & \begin{tabular}{|l|l|}
0.58 \\
\end{tabular} & \begin{tabular}{|l|}
0.55 \\
\end{tabular} & 0.62 & 0.58 & 0.52 & 0.45 & 0.42 & 0.42 & 0.36 & 0.23 & 0.10 & 0.06 & 0.00 & 0.00 \\
\hline$(\mathrm{T} *-11)$ & \begin{tabular}{|l|}
0.4 \\
\end{tabular} & \begin{tabular}{|l|}
0.36 \\
\end{tabular} & \begin{tabular}{|l|}
0.34 \\
\end{tabular} & 0.36 & 0.34 & 0.28 & 0.24 & 0.22 & 0.22 & 0.16 & 0.1 & 0 & 0 & 0 & 0 \\
\hline
\end{tabular}

\begin{tabular}{|c|c|c|c|c|c|c|c|c|c|c|c|c|c|c|c|}
\hline Rita (2005) & 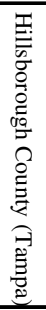 & 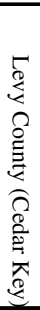 & 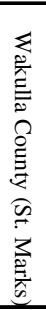 & 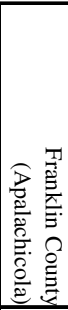 & 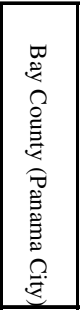 & 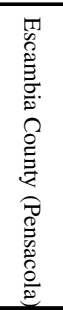 & 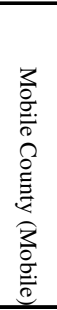 & 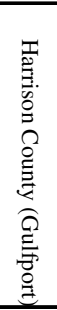 & 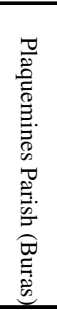 & 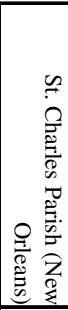 & 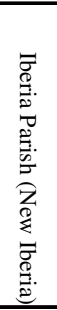 & 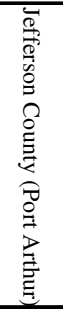 & 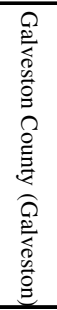 & 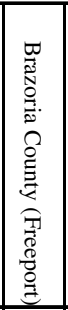 & 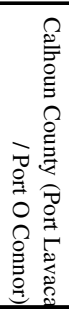 \\
\hline $\mathrm{T}^{*}$ & 0 & 0 & 0 & 0 & 0 & 0.06 & 0.12 & 0.15 & 0.15 & 0.24 & 1.38 & 2.58 & 1.92 & 1.11 & 0.27 \\
\hline$\left(\mathrm{T}^{*}-1\right)$ & 0 & 0 & 0 & 0 & 0 & 0.09 & 0.15 & 0.18 & 0.18 & \begin{tabular}{|l|}
0.33 \\
\end{tabular} & 0.90 & 1.59 & 1.44 & \begin{tabular}{|l|}
0.99 \\
\end{tabular} & 0.39 \\
\hline$\left(\mathrm{T}^{*}-2\right)$ & 0 & 0 & 0 & 0 & 0 & 0.11 & 0.22 & 0.25 & 0.29 & \begin{tabular}{|l|}
0.43 \\
\end{tabular} & 0.86 & 1.33 & 1.33 & 1.04 & 0.43 \\
\hline$\left(\mathrm{T}^{*}-3\right)$ & 0 & 0 & 0 & 0 & 0 & 0.11 & 0.19 & 0.27 & 0.38 & \begin{tabular}{|l}
0.50 \\
\end{tabular} & 0.84 & 1.18 & 1.18 & 1.03 & 0.65 \\
\hline$\left(T^{*}-4\right)$ & 0 & 0 & 0 & 0 & 0 & 0.11 & 0.23 & 0.34 & 0.46 & \begin{tabular}{|l|}
0.57 \\
\end{tabular} & 0.88 & 1.10 & 1.10 & 0.95 & 0.69 \\
\hline$\left(\mathrm{T}^{*}-5\right)$ & 0 & 0 & 0 & 0 & 0.075 & 0.15 & 0.26 & 0.34 & 0.52 & 0.56 & 0.79 & 0.97 & 0.97 & 0.90 & 0.67 \\
\hline$\left(\mathrm{T}^{*}-6\right)$ & 0 & 0 & 0 & 0 & \begin{tabular}{|l|}
0.081 \\
\end{tabular} & 0.16 & 0.28 & 0.41 & 0.61 & \begin{tabular}{|l|}
0.65 \\
\end{tabular} & 0.85 & 0.98 & 0.98 & \begin{tabular}{|l|}
0.94 \\
\end{tabular} & \begin{tabular}{|l|}
0.77 \\
\end{tabular} \\
\hline$\left(T^{*}-7\right)$ & 0 & 0 & 0 & 0 & 0 & 0.17 & 0.26 & 0.39 & 0.56 & 0.56 & 0.78 & 0.91 & 0.95 & 0.95 & \begin{tabular}{|l|}
0.86 \\
\end{tabular} \\
\hline$\left(\mathrm{T}^{*}-8\right)$ & 0 & 0 & 0 & 0 & 0 & 0.13 & 0.22 & 0.31 & 0.49 & 0.49 & \begin{tabular}{|l|}
0.67 \\
\end{tabular} & 0.76 & 0.85 & 0.85 & \begin{tabular}{|l|}
0.85 \\
\end{tabular} \\
\hline$\left(T^{*}-9\right)$ & 0 & 0 & 0 & 0 & 0 & 0.12 & 0.16 & 0.24 & 0.37 & 0.41 & 0.53 & 0.61 & 0.73 & 0.73 & 0.73 \\
\hline$\left(T^{*}-10\right)$ & 0 & 0 & 0 & 0 & 0 & 0.11 & 0.15 & 0.22 & 0.37 & 0.37 & 0.45 & 0.52 & 0.59 & \begin{tabular}{|l|}
0.63 \\
\end{tabular} & 0.63 \\
\hline$\left(\mathrm{T}^{*}-11\right)$ & 0 & 0 & 0 & 0 & \begin{tabular}{|l|l|}
0.075 \\
\end{tabular} & 0.11 & 0.19 & 0.26 & 0.38 & \begin{tabular}{|l|}
0.34 \\
\end{tabular} & 0.45 & 0.49 & 0.53 & 0.57 & 0.57 \\
\hline
\end{tabular}

\begin{tabular}{|c|c|c|c|c|c|c|c|c|c|c|c|c|c|c|c|}
\hline Bonnie (2004) & 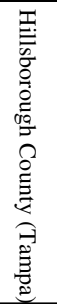 & 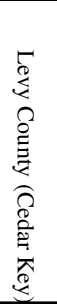 & 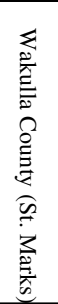 & 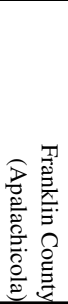 & 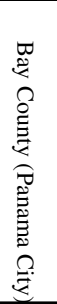 & 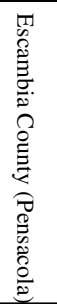 & 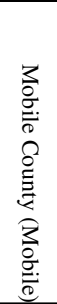 & 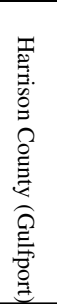 & 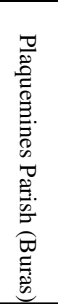 & 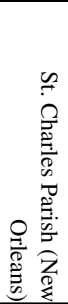 & 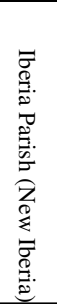 & 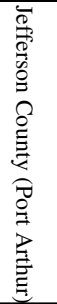 & 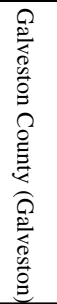 & 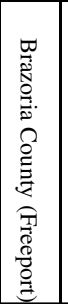 & 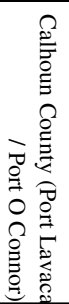 \\
\hline $\mathrm{T}^{*}$ & 0.00 & 0.00 & 0.00 & 0.00 & 0.00 & 0.00 & 0.00 & 0.00 & 0.00 & 0.00 & 0.00 & 0.00 & 0.00 & \begin{tabular}{|l|}
0.00 \\
\end{tabular} & 0.00 \\
\hline$\left(T^{*}-1\right)$ & 0.02 & 0.05 & 0.09 & 0.11 & 0.12 & 0.07 & 0.01 & 0.00 & 0.00 & 0.00 & 0.00 & 0.00 & 0.00 & \begin{tabular}{|l|}
0.00 \\
\end{tabular} & 0.00 \\
\hline$\left(T^{*}-2\right)$ & 0.07 & 0.13 & 0.20 & 0.24 & 0.25 & 0.17 & 0.05 & 0.01 & 0.00 & 0.00 & 0.00 & 0.00 & 0.00 & \begin{tabular}{|l|}
0.00 \\
\end{tabular} & 0.00 \\
\hline$\left(T^{*}-3\right)$ & 0.03 & 0.06 & 0.09 & 0.11 & 0.12 & 0.12 & 0.10 & 0.08 & 0.12 & 0.05 & 0.00 & 0.00 & 0.00 & \begin{tabular}{|l|}
0.00 \\
\end{tabular} & 0.00 \\
\hline$\left(T^{*}-4\right)$ & 0.00 & 0.00 & 0.00 & 0.00 & 0.00 & 0.00 & 0.00 & 0.00 & 0.00 & 0.00 & 0.00 & 0.00 & 0.00 & \begin{tabular}{|l|}
0.00 \\
\end{tabular} & 0.00 \\
\hline$\left(T^{*}-5\right)$ & 0.02 & 0.05 & 0.09 & 0.10 & 0.11 & 0.13 & 0.12 & 0.11 & 0.12 & 0.07 & 0.01 & 0.00 & 0.00 & \begin{tabular}{|l|}
0.00 \\
\end{tabular} & 0.00 \\
\hline$\left(\mathrm{T}^{*}-t\right.$ & 0.09 & 0.12 & 0.16 & 0.17 & 0.19 & 0.19 & 0.17 & 0.16 & 0.17 & 0.12 & 0.04 & 0.00 & 0.00 & \begin{tabular}{|l|l|}
0.00 \\
\end{tabular} & 0.00 \\
\hline$\left(T^{*}-7\right)$ & 0.10 & 0.13 & 0.15 & 0.16 & 0.17 & 0.18 & 0.17 & 0.17 & 0.19 & 0.16 & 0.10 & 0.01 & 0.00 & \begin{tabular}{|l|}
0.00 \\
\end{tabular} & 0.00 \\
\hline$\left(T^{*}-8\right)$ & 0.07 & 0.08 & 0.10 & 0.10 & 0.11 & 0.11 & 0.11 & 0.11 & 0.11 & 0.10 & 0.08 & 0.04 & 0.03 & \begin{tabular}{|l|}
0.02 \\
\end{tabular} & 0.00 \\
\hline & 0.02 & 0.02 & 0.03 & 0.0 & 0.03 & 0.03 & 0.03 & 0.04 & 0.04 & 0.04 & 0.03 & 0.02 & 0.01 & \begin{tabular}{|l|l}
0.01 \\
\end{tabular} & 0.00 \\
\hline$\left(T^{*}-10\right)$ & 0.01 & 0.01 & 0.01 & 0.02 & 0.02 & 0.02 & 0.02 & 0.02 & 0.02 & 0.02 & 0.02 & 0.02 & 0.01 & 0.01 & 0.01 \\
\hline$\left(\mathrm{T}^{*}-11\right)$ & & & & & & & & & & & & & & & \\
\hline
\end{tabular}




\begin{tabular}{|c|c|c|c|c|c|c|c|c|c|c|c|c|c|c|c|}
\hline arley (2004) & 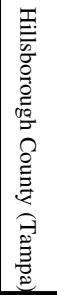 & 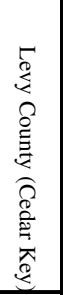 & 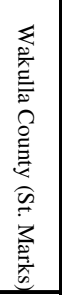 & 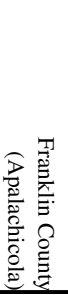 & 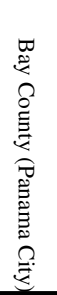 & 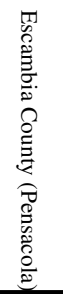 & 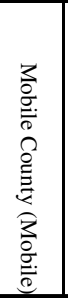 & 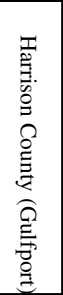 & 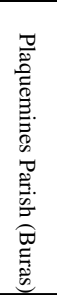 & 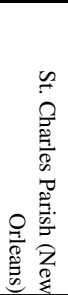 & 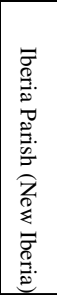 & 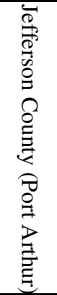 & 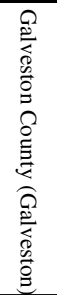 & 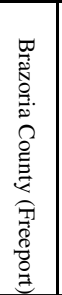 & 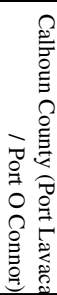 \\
\hline $\mathrm{T}^{*}$ & 2.31 & 1.98 & 1.00 & 0.39 & 0.11 & 0.00 & 0.00 & 0.00 & 0.00 & 0.00 & 0.00 & 0.00 & 0.00 & 0.00 & 0.00 \\
\hline$\left(T^{*}-1\right)$ & \begin{tabular}{|l|}
1.41 \\
\end{tabular} & 1.32 & 1.02 & 0.84 & 0.60 & 0.09 & 0.00 & 0.00 & 0.00 & 0.00 & 0.00 & 0.00 & 0.00 & 0.00 & 0.00 \\
\hline$\left(\mathrm{T}^{*}-2\right)$ & \begin{tabular}{|l|}
1.31 \\
\end{tabular} & 1.13 & 0.70 & 0.46 & 0.24 & 0.00 & 0.00 & 0.00 & 0.00 & 0.00 & 0.00 & 0.00 & 0.00 & 0.00 & 0.00 \\
\hline$(\mathrm{T} *-3)$ & 1.19 & 1.06 & 0.79 & 0.61 & 0.43 & 0.09 & 0.00 & 0.00 & 0.00 & 0.00 & 0.00 & 0.00 & 0.00 & 0.00 & 0.00 \\
\hline$\left(T^{*}-4\right)$ & 0.86 & 0.79 & 0.60 & 0.50 & 0.36 & 0.10 & 0.00 & 0.00 & 0.00 & 0.00 & 0.00 & 0.00 & 0.00 & 0.00 & 0.00 \\
\hline$\left(\mathrm{T}^{*}-5\right)$ & \begin{tabular}{|l|}
0.86 \\
\end{tabular} & 0.81 & 0.65 & 0.58 & 0.43 & 0.14 & 0.05 & 0.00 & 0.00 & 0.00 & 0.00 & 0.00 & 0.00 & 0.00 & 0.00 \\
\hline$(T *-6)$ & 07 & 0.64 & 0.46 & 0.36 & 0.28 & 0.06 & 0.00 & 0.00 & 0.00 & 0.00 & 0.00 & 0.00 & 0.00 & 0.00 & 0.00 \\
\hline$(\mathrm{T} *-7)$ & 0.58 & 0.56 & 0.46 & 0.40 & 0.34 & 0.14 & 0.04 & 0.00 & 0.00 & 0.00 & 0.00 & 0.00 & 0.00 & 0.00 & 0.00 \\
\hline$\left(\mathrm{T}^{*}-8\right)$ & \begin{tabular}{|l|}
0.39 \\
\end{tabular} & 0.36 & 0.29 & 0.25 & 0.21 & 0.07 & $\begin{array}{ll}0.03 \\
\end{array}$ & 0.00 & 0.00 & 0.00 & 0.00 & 0.00 & 0.00 & 0.00 & 0.00 \\
\hline$\left(\mathrm{T}^{*}-9\right)$ & \begin{tabular}{|l|}
0.29 \\
\end{tabular} & 0.27 & 0.23 & 0.20 & 0.16 & 0.07 & 0.02 & 0.00 & 0.00 & 0.00 & 0.00 & 0.00 & 0.00 & 0.00 & 0.00 \\
\hline$\left(\mathrm{T}^{*}-10\right)$ & 0.28 & 0.25 & 0.20 & 0.21 & 0.20 & 0.12 & $\begin{array}{ll}0.09 \\
\end{array}$ & 0.07 & 0.05 & 0.04 & 0.00 & 0.00 & 0.00 & 0.00 & 0.00 \\
\hline$\left(\mathrm{T}^{*}-11\right)$ & 0.21 & 0.18 & 0.12 & 0.16 & 0.14 & 0.09 & 0.05 & 0.04 & 0.05 & 0.00 & 0.00 & 0.00 & 0.00 & 0.00 & 0.00 \\
\hline
\end{tabular}

\begin{tabular}{|c|c|c|c|c|c|c|c|c|c|c|c|c|c|c|c|}
\hline Frances (2004) & 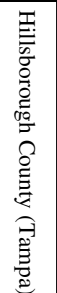 & 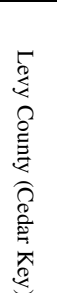 & 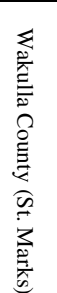 & 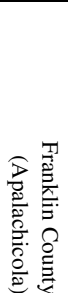 & 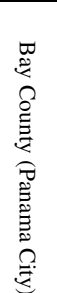 & 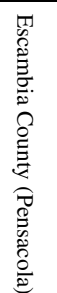 & 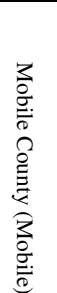 & 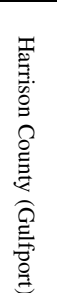 & 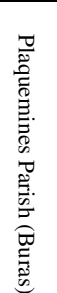 & 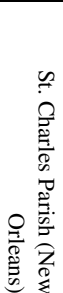 & 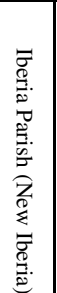 & 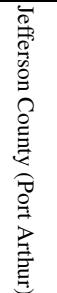 & 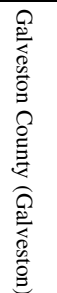 & 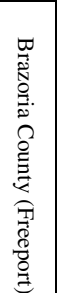 & 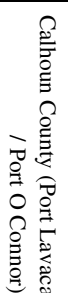 \\
\hline $\mathrm{T}^{*}$ & 0.00 & 0.00 & 0.00 & 0.00 & 0.00 & 0.00 & 0.00 & 0.00 & 0.00 & 0.00 & 0.00 & 0.00 & 0.00 & 0.00 & 0.00 \\
\hline$\left(\mathrm{T}^{*}-1\right)$ & \begin{tabular}{|l|}
0.59 \\
\end{tabular} & 0.59 & 0.49 & 0.50 & 0.37 & 0.14 & 0.08 & 0.04 & 0.00 & 0.00 & 0.00 & 0.00 & 0.00 & 0.00 & 0.00 \\
\hline$\left(\mathrm{T}^{*}-2\right)$ & 0.56 & 0.56 & 0.27 & 0.25 & 0.21 & 0.12 & 0.09 & 0.06 & 0.03 & 0.03 & 0.00 & 0.00 & 0.00 & 0.00 & 0.00 \\
\hline$\left(\mathrm{T}^{*}-3\right)$ & \begin{tabular}{|l|}
0.56 \\
\end{tabular} & 0.43 & 0.28 & 0.28 & 0.24 & 0.15 & 0.10 & 0.06 & 0.03 & 0.03 & 0.00 & 0.00 & 0.00 & 0.00 & 0.00 \\
\hline$\left(T^{*}-4\right)$ & 0.36 & 0.34 & 0.22 & 0.20 & 0.17 & 0.12 & 0.09 & 0.07 & 0.03 & 0.03 & 0.01 & 0.00 & 0.00 & 0.00 & 0.00 \\
\hline$\left(T^{*}-5\right)$ & 0.23 & 0.18 & 0.11 & 0.10 & 0.08 & 0.06 & 0.05 & 0.04 & 0.02 & 0.02 & 0.01 & 0.00 & 0.00 & 0.00 & 0.00 \\
\hline$\left(T^{*}-6\right)$ & 0.15 & 0.14 & 0.11 & 0.09 & 0.08 & 0.06 & 0.05 & 0.05 & 0.03 & 0.03 & 0.02 & 0.01 & 0.00 & 0.00 & 0.00 \\
\hline$\left(T^{*}-7\right)$ & 0.15 & 0.13 & 0.10 & 0.09 & 0.09 & 0.07 & 0.06 & 0.06 & 0.04 & 0.04 & 0.03 & 0.01 & 0.01 & 0.00 & 0.00 \\
\hline$\left(\mathrm{T}^{*}-8\right)$ & 0.12 & 0.11 & 0.09 & 0.08 & 0.08 & 0.07 & 0.06 & 0.05 & 0.04 & 0.04 & 0.03 & 0.01 & 0.01 & 0.00 & 0.00 \\
\hline$\left(\mathrm{T}^{*}-9\right)$ & 0.00 & 0.00 & 0.00 & 0.00 & 0.00 & 0.00 & 0.00 & 0.00 & 0.00 & 0.00 & 0.00 & 0.00 & 0.00 & 0.00 & 0.00 \\
\hline$\left(\mathrm{T}^{*}-10\right)$ & 0.00 & 0.00 & 0.00 & 0.00 & 0.00 & 0.0 & 0.00 & 0.00 & 0.00 & 0.00 & 0.00 & 0.00 & 0.00 & 0.00 & 0.00 \\
\hline$\left(\mathrm{T}^{*}-11\right)$ & \begin{tabular}{|l|}
0.00 \\
\end{tabular} & 0.00 & 0.00 & 0.00 & 0.00 & 0.00 & 0.00 & 0.00 & 0.00 & 0.00 & 0.00 & 0.00 & 0.00 & 0.00 & 0.00 \\
\hline
\end{tabular}

\begin{tabular}{|c|c|c|c|c|c|c|c|c|c|c|c|c|c|c|c|}
\hline (2004) & 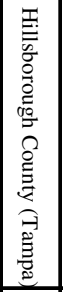 & 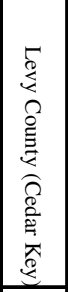 & 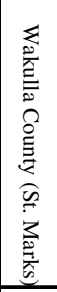 & 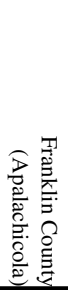 & 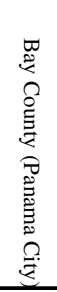 & 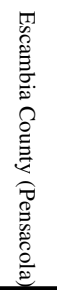 & 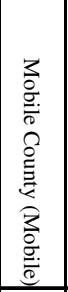 & 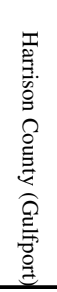 & 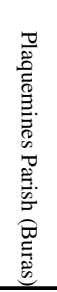 & 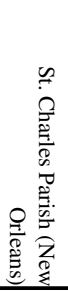 & 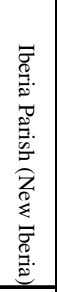 & 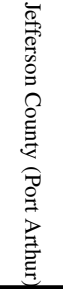 & 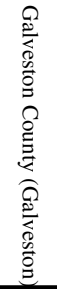 & 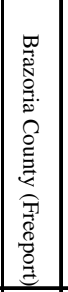 & 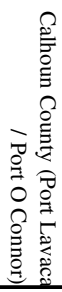 \\
\hline $\mathrm{T}$ & \begin{tabular}{|l|l|}
0.07 \\
\end{tabular} & \begin{tabular}{|l|}
0.18 \\
\end{tabular} & \begin{tabular}{|l|}
0.29 \\
\end{tabular} & \begin{tabular}{|l|}
0.25 \\
\end{tabular} & 0.36 & 2.66 & \begin{tabular}{|l|}
2.98 \\
\end{tabular} & \begin{tabular}{|l|}
2.18 \\
\end{tabular} & 0.98 & 0.22 & 0.00 & 0.00 & 0.00 & \begin{tabular}{|l|}
0.00 \\
\end{tabular} & 0.00 \\
\hline (T) & 11 & \begin{tabular}{|l|}
0.22 \\
\end{tabular} & \begin{tabular}{|l|}
0.40 \\
\end{tabular} & \begin{tabular}{|l|}
0.40 \\
\end{tabular} & 0.54 & 1.62 & \begin{tabular}{|l|}
2.12 \\
\end{tabular} & \begin{tabular}{|l|}
1.87 \\
\end{tabular} & 1.44 & 0.58 & 0.00 & 0.00 & 0.00 & \begin{tabular}{|l|}
0.00 \\
\end{tabular} & 0.00 \\
\hline & 1 & \begin{tabular}{|l|}
0.22 \\
\end{tabular} & \begin{tabular}{|l|}
0.40 \\
\end{tabular} & 0.5 & 0.6 & 1.37 & \begin{tabular}{|l|}
1.55 \\
\end{tabular} & \begin{tabular}{|l|}
1.40 \\
\end{tabular} & 1.37 & 0.72 & \begin{tabular}{|l|}
0.11 \\
\end{tabular} & 0.00 & 0.00 & \begin{tabular}{|l|}
0.00 \\
\end{tabular} & 0.00 \\
\hline 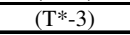 & 5 & \begin{tabular}{|l|}
0.30 \\
\end{tabular} & \begin{tabular}{|l|}
0.53 \\
\end{tabular} & 0.65 & 0.80 & 1.14 & \begin{tabular}{|l|}
1.22 \\
\end{tabular} & \begin{tabular}{|l|}
1.14 \\
\end{tabular} & 1.10 & $\begin{array}{l}0.80 \\
\end{array}$ & \begin{tabular}{|l|}
0.23 \\
\end{tabular} & 0.00 & 0.00 & \begin{tabular}{|l|}
0.00 \\
\end{tabular} & 0.00 \\
\hline & & \begin{tabular}{|l|}
0.24 \\
\end{tabular} & 0.4 & & 0.7 & & \begin{tabular}{|l|}
1.24 \\
\end{tabular} & 1.24 & 1.28 & 1.04 & 5 & 0.12 & 0.00 & .00 & .00 \\
\hline & & \begin{tabular}{|l|}
0.22 \\
\end{tabular} & \begin{tabular}{|l|}
0.45 \\
\end{tabular} & 0.5 & 0.6 & 0. & 0.97 & \begin{tabular}{|l|}
0.97 \\
\end{tabular} & 1.01 & 0.8 & \begin{tabular}{|l|}
0.49 \\
\end{tabular} & 0.15 & 0.07 & \begin{tabular}{|l|}
0.00 \\
\end{tabular} & 0.00 \\
\hline+1 & 11 & \begin{tabular}{|l|}
0.22 \\
\end{tabular} & \begin{tabular}{|l|}
0.41 \\
\end{tabular} & \begin{tabular}{|l|l}
0.49 \\
\end{tabular} & 0.60 & 0.79 & \begin{tabular}{|l|}
0.90 \\
\end{tabular} & 0.94 & 1.01 & 0.86 & 0.60 & 0.26 & 0.15 & \begin{tabular}{|l|}
0.07 \\
\end{tabular} & 0.00 \\
\hline & & & & & 0.5 & & & & 0.93 & & \begin{tabular}{|l|}
0.59 \\
\end{tabular} & 0.26 & 0.15 & \begin{tabular}{|l|}
0.07 \\
\end{tabular} & 0.00 \\
\hline$*_{-\zeta}^{-8}$ & 22 & \begin{tabular}{|l|}
0.26 \\
\end{tabular} & \begin{tabular}{|l|l|}
0.37 \\
\end{tabular} & 0.45 & 0.52 & 0.59 & \begin{tabular}{|l|}
0.63 \\
\end{tabular} & \begin{tabular}{|l|l|}
0.67 \\
\end{tabular} & 0.74 & 0.67 & 0.59 & 0.37 & 0.26 & \begin{tabular}{|l|l|}
0.19 \\
\end{tabular} & 0.07 \\
\hline & & \begin{tabular}{|l|}
0.33 \\
\end{tabular} & \begin{tabular}{|l|l|}
0.48 \\
\end{tabular} & 0.5 & 0.6 & & \begin{tabular}{|l|}
0.71 \\
\end{tabular} & 0.74 & 0.78 & 0.67 & 0.48 & 0.26 & .15 & \begin{tabular}{|l|}
0.11 \\
\end{tabular} & 0.00 \\
\hline & & 0.2 & \begin{tabular}{|l|}
0.4 \\
\end{tabular} & 0.48 & 0.5 & 0.5 & \begin{tabular}{|l|}
0.56 \\
\end{tabular} & 0.59 & 0.63 & 0.59 & 0.48 & 0.30 & .22 & \begin{tabular}{|l|l|}
0.15 \\
\end{tabular} & .07 \\
\hline$\left(\mathrm{T}^{*}-11\right)$ & \begin{tabular}{|l|}
0.24 \\
\end{tabular} & \begin{tabular}{|l|}
0.24 \\
\end{tabular} & \begin{tabular}{|l|l|}
0.30 \\
\end{tabular} & 0.36 & 0.39 & 0.42 & \begin{tabular}{|l|}
0.42 \\
\end{tabular} & 0.42 & 0.48 & 0.42 & \begin{tabular}{|l|}
0.36 \\
\end{tabular} & 0.21 & 0.18 & \begin{tabular}{|l|}
0.15 \\
\end{tabular} & 0.09 \\
\hline
\end{tabular}




\begin{tabular}{|c|c|c|c|c|c|c|c|c|c|c|c|c|c|c|c|}
\hline Matthew (2004) & 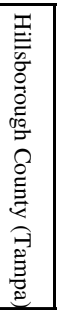 & 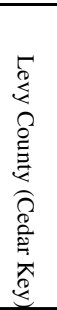 & 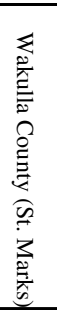 & 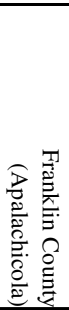 & 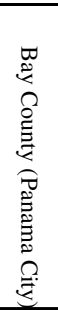 & 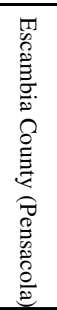 & 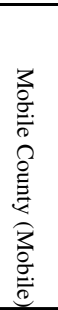 & 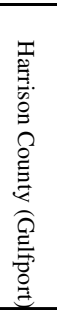 & 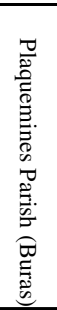 & 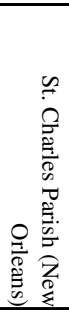 & 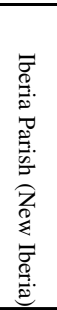 & 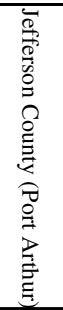 & 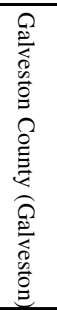 & 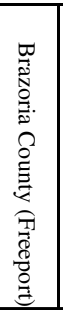 & 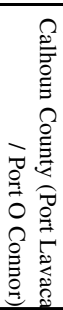 \\
\hline $\mathrm{T}^{*}$ & 0.00 & 0.00 & 0.00 & 0.00 & 0.00 & 0.00 & 0.00 & 0.00 & 0.00 & 0.00 & 0.00 & 0.00 & 0.00 & 0.00 & 0.00 \\
\hline$\left(\mathrm{T}^{*}-1\right)$ & 0.00 & 0.00 & 0.00 & 0.00 & 0.00 & 0.00 & 0.00 & 0.00 & 0.00 & 0.00 & 0.00 & 0.00 & 0.00 & 0.00 & 0.00 \\
\hline$\left(T^{*}-2\right)$ & 0.00 & 0.00 & 0.00 & 0.00 & 0.00 & 0.00 & 0.00 & 0.00 & 0.00 & 0.00 & 0.00 & 0.00 & 0.00 & 0.00 & 0.00 \\
\hline$(\mathrm{T} *-3)$ & 0.00 & 0.00 & 0.00 & 0.00 & 0.00 & 0.00 & 0.00 & 0.00 & 0.00 & 0.00 & 0.00 & 0.00 & 0.00 & 0.00 & 0.00 \\
\hline$\left(T^{*}-4\right)$ & 0.00 & 0.00 & 0.00 & 0.00 & 0.00 & 0.00 & 0.00 & 0.00 & 0.00 & 0.00 & 0.00 & 0.00 & 0.00 & 0.00 & 0.00 \\
\hline$\left(T^{*}-5\right)$ & 0.00 & 0.00 & 0.00 & 0.00 & 0.00 & 0.00 & 0.00 & 0.00 & 0.00 & 0.00 & 0.00 & 0.00 & 0.00 & 0.00 & 0.00 \\
\hline$\left(T^{*}-6\right)$ & 0.00 & 0.00 & 0.00 & 0.00 & 0.00 & 0.00 & 0.00 & 0.00 & 0.00 & 0.00 & 0.00 & 0.00 & 0.00 & 0.00 & 0.00 \\
\hline$\left(T^{*}-7\right)$ & & & & & & & & & & & & & & & \\
\hline \multicolumn{16}{|l|}{$\left(T^{*}-8\right)$} \\
\hline \multirow{2}{*}{\multicolumn{16}{|c|}{$\left(T^{*}-9\right)$}} \\
\hline \multirow{2}{*}{\multicolumn{16}{|c|}{$\frac{\left(\mathrm{T}^{*}-10\right)}{\left(\mathrm{T}^{*}-11\right)}$}} \\
\hline & & & & & & & & & & & & & & & \\
\hline & & & & & & & & & & & & & & & \\
\hline
\end{tabular}

\begin{tabular}{|c|c|c|c|c|c|c|c|c|c|c|c|c|c|c|c|}
\hline laudette (2003) & 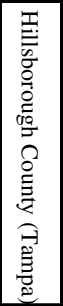 & 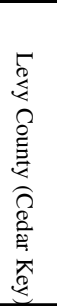 & 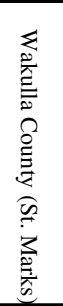 & 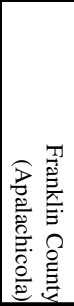 & 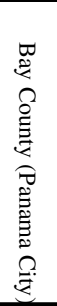 & 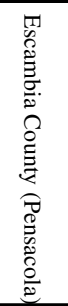 & 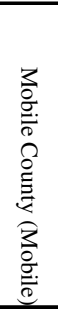 & 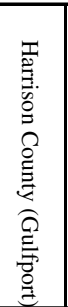 & 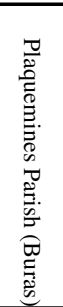 & 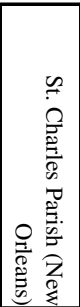 & 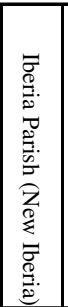 & 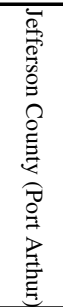 & 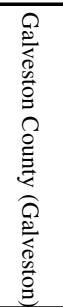 & 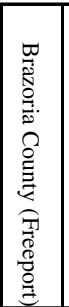 & 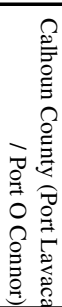 \\
\hline $\mathrm{T}^{*}$ & 0.00 & 0.00 & 0.00 & 0.00 & 0.00 & 0.00 & 0.00 & 0.00 & 0.00 & \begin{tabular}{|l|}
0.00 \\
\end{tabular} & \begin{tabular}{|l|}
0.00 \\
\end{tabular} & 0.01 & 0.41 & \begin{tabular}{|l|}
0.48 \\
\end{tabular} & 0.52 \\
\hline$\left(T^{*}-1\right)$ & 0.00 & 0.00 & 0.00 & 0.00 & 0.00 & 0.00 & 0.00 & 0.00 & 0.00 & 0.00 & \begin{tabular}{|l|}
0.00 \\
\end{tabular} & 0.11 & 0.29 & \begin{tabular}{|l|}
0.33 \\
\end{tabular} & 0.30 \\
\hline$\left(\mathrm{T}^{*}-2\right)$ & 0.00 & 0.00 & 0.00 & 0.00 & 0.00 & 0.00 & 0.00 & 0.00 & 0.00 & 0.00 & \begin{tabular}{|l|}
0.03 \\
\end{tabular} & 0.11 & 0.19 & 0.21 & 0.19 \\
\hline$\left(\mathrm{T}^{*}-3\right)$ & 0.00 & 0.00 & 0.00 & 0.00 & 0.00 & 0.00 & 0.00 & 0.00 & 0.00 & 0.00 & 0.05 & 0.14 & 0.19 & \begin{tabular}{|l|}
0.20 \\
\end{tabular} & 0.20 \\
\hline$\left(T^{*}-4\right)$ & \begin{tabular}{|l|}
0.00 \\
\end{tabular} & 0.00 & 0.00 & 0.00 & 0.00 & 0.00 & 0.00 & 0.00 & \begin{tabular}{|l|}
0.00 \\
\end{tabular} & 0.01 & \begin{tabular}{|l|}
0.03 \\
\end{tabular} & 0.08 & 0.12 & \begin{tabular}{|l|}
0.14 \\
\end{tabular} & 0.15 \\
\hline$\left(\mathrm{T}^{*}-5\right)$ & 0.00 & 0.00 & 0.00 & 0.00 & 0.00 & 0.00 & 0.00 & 0.00 & 0.00 & 0.01 & \begin{tabular}{|l|}
0.03 \\
\end{tabular} & 0.07 & 0.10 & \begin{tabular}{|l|}
0.11 \\
\end{tabular} & 0.13 \\
\hline$\left(T^{*}-6\right)$ & 0.00 & 0.00 & 0.00 & 0.00 & 0.00 & 0.00 & 0.00 & 0.00 & 0.00 & 0.00 & \begin{tabular}{|l|}
0.02 \\
\end{tabular} & 0.05 & 0.08 & \begin{tabular}{|l|}
0.10 \\
\end{tabular} & 0.12 \\
\hline$(\mathrm{T} *-7)$ & 0.00 & 0.00 & 0.00 & 0.00 & 0.00 & 0.00 & 0.00 & 0.00 & 0.00 & 0.00 & \begin{tabular}{|l|}
0.02 \\
\end{tabular} & 0.04 & 0.08 & \begin{tabular}{|l|}
0.09 \\
\end{tabular} & 0.12 \\
\hline$(\mathrm{T} *-8)$ & 0.00 & 0.00 & 0.00 & 0.00 & 0.00 & 0.00 & 0.00 & 0.00 & 0.00 & 0.00 & 0.01 & 0.03 & 0.04 & 0.05 & 0.06 \\
\hline$\left(T^{*}-9\right)$ & 0.00 & 0.00 & 0.00 & 0.00 & 0.00 & 0.00 & 0.00 & 0.00 & 0.01 & 0.01 & 0.01 & 0.03 & 0.04 & \begin{tabular}{|l|}
0.06 \\
\end{tabular} & 0.07 \\
\hline$(T *-10)$ & 0.00 & 0.00 & 0.00 & 0.00 & 0.00 & 0.00 & 0.00 & 0.00 & 0.00 & 0.01 & \begin{tabular}{|l|}
0.01 \\
\end{tabular} & 0.02 & 0.03 & \begin{tabular}{|l|}
0.03 \\
\end{tabular} & 0.04 \\
\hline$\left(\mathrm{T}^{*}-11\right)$ & 0.00 & 0.00 & 0.00 & 0.00 & 0.00 & 0.00 & 0.00 & 0.00 & 0.00 & 0.00 & \begin{tabular}{|l|}
0.01 \\
\end{tabular} & 0.02 & 0.02 & \begin{tabular}{|l|}
0.03 \\
\end{tabular} & 0.04 \\
\hline
\end{tabular}

\begin{tabular}{|c|c|c|c|c|c|c|c|c|c|c|c|c|c|c|c|}
\hline Lili (2002) & 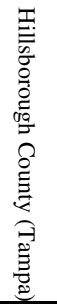 & 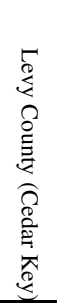 & 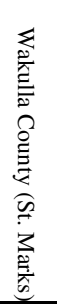 & 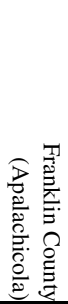 & 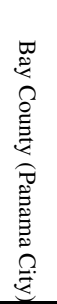 & 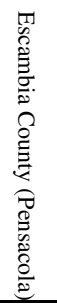 & 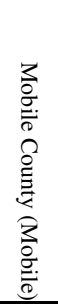 & 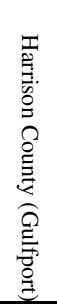 & 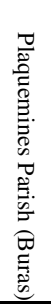 & 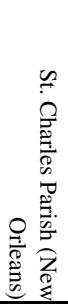 & 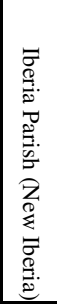 & 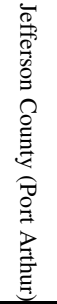 & 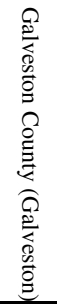 & 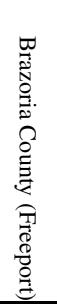 & 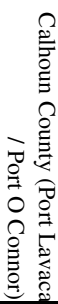 \\
\hline $\mathrm{T}^{*}$ & 0.00 & 0.00 & 0.00 & 0.00 & 0.00 & 0.00 & 0.09 & 0.15 & 0.09 & 0.93 & 2.49 & 0.63 & 0.00 & 0.00 & 0.00 \\
\hline$\left(T^{*}-1\right)$ & 0.00 & 0.00 & 0.00 & 0.00 & 0.00 & 0.08 & 0.44 & 0.88 & 1.12 & 1.88 & 2.72 & 0.64 & 0.00 & 0.00 & 0.00 \\
\hline$\left(\mathrm{T}^{*}-2\right)$ & 0.00 & 0.00 & 0.00 & 0.00 & 0.00 & 0.24 & 0.56 & 0.76 & 0.80 & 1.00 & 1.48 & 1.24 & 0.76 & 0.36 & 0.00 \\
\hline$\left(\mathrm{T}^{*}-3\right)$ & 0.00 & 0.00 & 0.07 & 0.10 & 0.13 & 0.33 & 0.50 & 0.60 & 0.66 & 0.76 & 0.96 & 0.89 & 0.73 & 0.56 & 0.27 \\
\hline$\left(T^{*}-4\right)$ & 0.00 & 0.00 & 0.09 & 0.09 & 0.15 & 0.33 & 0.42 & 0.49 & 0.55 & 0.64 & 0.79 & 0.76 & 0.64 & 0.52 & 0.30 \\
\hline$\left(\mathrm{T}^{*}-5\right)$ & 0.00 & 0.00 & 0.06 & 0.09 & 0.12 & 0.27 & 0.36 & 0.42 & 0.52 & 0.58 & 0.70 & 0.70 & 0.64 & 0.55 & 0.39 \\
\hline$\left(T^{*}-6\right)$ & 0.00 & 0.00 & 0.06 & 0.06 & 0.12 & 0.24 & 0.33 & 0.39 & 0.51 & 0.55 & 0.64 & 0.64 & 0.61 & 0.55 & 0.39 \\
\hline$\left(T^{*}-7\right)$ & 0.00 & 0.00 & 0.06 & 0.09 & 0.12 & 0.24 & 0.30 & 0.39 & 0.48 & 0.48 & 0.55 & 0.55 & 0.55 & 0.51 & 0.42 \\
\hline$(\mathrm{T} *-8)$ & 0.00 & 0.00 & 0.09 & 0.15 & 0.18 & 0.30 & 0.36 & 0.42 & 0.48 & 0.48 & 0.51 & 0.48 & 0.45 & 0.42 & 0.33 \\
\hline$\left(T^{*}-9\right)$ & 0.06 & 0.06 & 0.06 & 0.12 & 0.15 & 0.24 & 0.27 & 0.36 & 0.45 & 0.42 & 0.45 & 0.45 & 0.42 & 0.42 & 0.36 \\
\hline$\left(T^{*}-10\right)$ & 0.06 & 0.06 & 0.06 & 0.12 & 0.12 & 0.18 & 0.21 & 0.27 & 0.36 & 0.33 & 0.36 & 0.36 & 0.36 & 0.36 & 0.30 \\
\hline$(\mathrm{T} *-11)$ & 0.15 & 0.12 & 0.12 & 0.18 & 0.21 & 0.24 & 0.24 & 0.30 & 0.39 & 0.36 & 0.39 & 0.36 & 0.36 & 0.33 & 0.27 \\
\hline
\end{tabular}




\begin{tabular}{|c|c|c|c|c|c|c|c|c|c|c|c|c|c|c|c|}
\hline Earl (1998) & 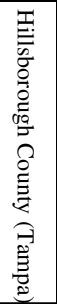 & 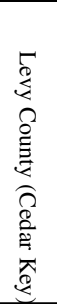 & 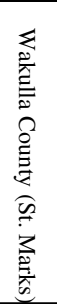 & 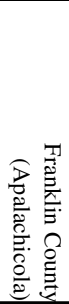 & 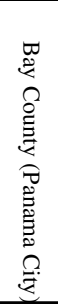 & 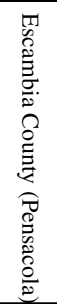 & 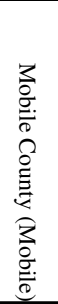 & 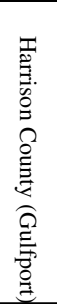 & 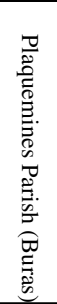 & 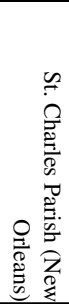 & 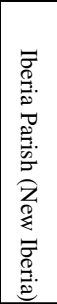 & 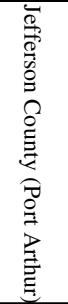 & 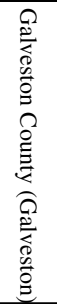 & 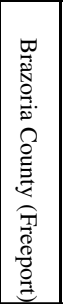 & 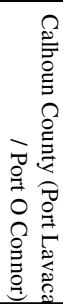 \\
\hline $\mathrm{T}^{*}$ & 0.14 & 0.26 & 0.51 & \begin{tabular}{|l|}
0.69 \\
\end{tabular} & 0.99 & 0.58 & 0.03 & 0.00 & 0.00 & \begin{tabular}{|l|}
0.00 \\
\end{tabular} & 0.00 & 0.00 & 0.00 & 0.00 & 0.00 \\
\hline$\left(\mathrm{T}^{*}-1\right)$ & 0.26 & 0.38 & 0.77 & 1.03 & 1.21 & 0.89 & 0.20 & 0.12 & 0.08 & \begin{tabular}{|l|}
0.04 \\
\end{tabular} & 0.00 & 0.00 & 0.00 & 0.00 & 0.00 \\
\hline$\left(\mathrm{T}^{*}-2\right)$ & 0.19 & 0.32 & 0.56 & 0.74 & 0.82 & 0.60 & 0.11 & 0.05 & 0.05 & \begin{tabular}{|l|}
0.03 \\
\end{tabular} & 0.00 & 0.00 & 0.00 & 0.00 & 0.00 \\
\hline$\left(\mathrm{T}^{*}-3\right)$ & 0.03 & 0.04 & 0.08 & \begin{tabular}{|l|}
0.09 \\
\end{tabular} & 0.11 & 0.15 & 0.15 & 0.17 & 0.26 & \begin{tabular}{|l|}
0.15 \\
\end{tabular} & 0.08 & 0.04 & 0.03 & 0.02 & 0.02 \\
\hline$\left(\mathrm{T}^{*}-4\right)$ & 0.02 & 0.03 & 0.05 & 0.06 & 0.08 & 0.11 & 0.13 & 0.15 & 0.24 & \begin{tabular}{|l|}
0.18 \\
\end{tabular} & 0.13 & 0.06 & 0.05 & \begin{tabular}{|l|}
0.04 \\
\end{tabular} & 0.02 \\
\hline$\left(\mathrm{T}^{*}-5\right)$ & 0.02 & 0.04 & 0.06 & 0.08 & 0.08 & 0.12 & 0.13 & 0.15 & 0.18 & \begin{tabular}{|l|}
0.18 \\
\end{tabular} & 0.22 & 0.14 & 0.10 & 0.07 & 0.03 \\
\hline$\left(T^{*}-6\right)$ & 0.00 & 0.03 & 0.06 & \begin{tabular}{|l|}
0.07 \\
\end{tabular} & 0.09 & 0.14 & 0.18 & 0.21 & 0.24 & \begin{tabular}{|l|}
0.26 \\
\end{tabular} & 0.30 & 0.27 & 0.27 & 0.26 & 0.21 \\
\hline$\left(T^{*}-7\right)$ & 0.00 & 0.00 & 0.02 & 0.02 & 0.03 & 0.05 & 0.06 & 0.07 & 0.07 & 0.08 & \begin{tabular}{|l|}
0.09 \\
\end{tabular} & 0.09 & 0.09 & 0.08 & 0.07 \\
\hline$\left(\mathrm{T}^{*}-8\right)$ & 0.04 & 0.05 & 0.06 & 0.07 & 0.07 & 0.08 & 0.09 & 0.10 & 0.11 & \begin{tabular}{|l|}
0.10 \\
\end{tabular} & 0.10 & 0.08 & 0.07 & 0.06 & 0.03 \\
\hline$(\mathrm{T} *-9)$ & 0.03 & 0.04 & 0.05 & 0.06 & 0.07 & 0.08 & 0.09 & 0.10 & 0.11 & \begin{tabular}{|l|}
0.11 \\
\end{tabular} & 0.11 & 0.08 & 0.07 & 0.05 & 0.03 \\
\hline$\left(T^{*}-10\right)$ & & & & & & & & & & & & & & & \\
\hline$\left(\mathrm{T}^{*}-11\right)$ & & & & & & & & & & & & & & & \\
\hline
\end{tabular}

\begin{tabular}{|c|c|c|c|c|c|c|c|c|c|c|c|c|c|c|c|}
\hline Georges (1998) & 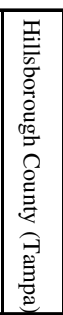 & 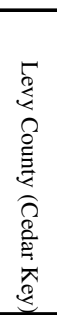 & 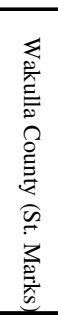 & 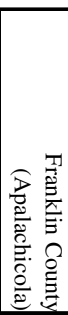 & 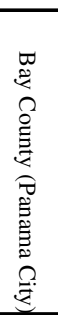 & 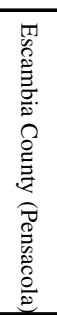 & 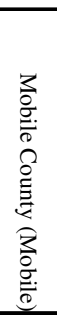 & 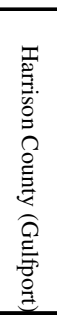 & 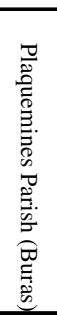 & 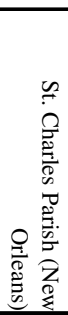 & 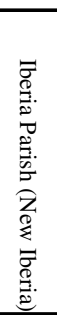 & 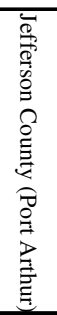 & 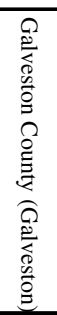 & 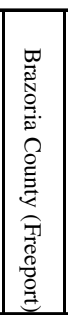 & 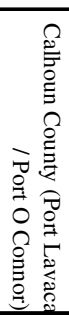 \\
\hline $\mathrm{T}^{*}$ & 0.08 & 0.10 & \begin{tabular}{|l|l|}
0.18 \\
\end{tabular} & 0.24 & 0.30 & 1.18 & 1.72 & 1.98 & 1.98 & 0.58 & 0.36 & 0.20 & 0.12 & 0.10 & 0.06 \\
\hline$\left(\mathrm{T}^{*}-1\right)$ & 0.10 & 0.15 & 0.22 & \begin{tabular}{|l|}
0.29 \\
\end{tabular} & 0.34 & 0.51 & 0.71 & 1.17 & 2.41 & 0.80 & \begin{tabular}{|l|}
0.46 \\
\end{tabular} & 0.24 & 0.15 & 0.12 & 0.05 \\
\hline$\left(T^{*}-2\right)$ & 0.08 & 0.11 & 0.21 & 0.32 & 0.40 & 0.62 & 0.73 & 0.89 & 1.15 & 0.86 & 0.62 & 0.35 & 0.21 & \begin{tabular}{|l|l|}
0.16 \\
\end{tabular} & 0.08 \\
\hline$\left(\mathrm{T}^{*}-3\right)$ & 0.08 & 0.11 & \begin{tabular}{|l|l|}
0.19 \\
\end{tabular} & \begin{tabular}{|l|}
0.32 \\
\end{tabular} & 0.40 & 0.59 & 0.67 & 0.78 & 0.99 & 0.75 & 0.59 & 0.38 & 0.24 & \begin{tabular}{|l|}
0.19 \\
\end{tabular} & 0.11 \\
\hline$\left(\mathrm{T}^{*}-4\right)$ & 0.11 & 0.13 & 0.24 & \begin{tabular}{|l|}
0.34 \\
\end{tabular} & 0.40 & 0.53 & 0.61 & 0.69 & 0.77 & 0.69 & 0.56 & 0.37 & 0.24 & \begin{tabular}{|l|}
0.19 \\
\end{tabular} & 0.11 \\
\hline$\left(T^{*}-5\right)$ & 0.16 & 0.19 & 0.24 & \begin{tabular}{|l|}
0.34 \\
\end{tabular} & 0.40 & 0.50 & 0.56 & 0.66 & 0.82 & 0.72 & 0.56 & 0.37 & 0.24 & \begin{tabular}{|l|}
0.19 \\
\end{tabular} & 0.11 \\
\hline$\left(T^{*}-6\right)$ & 0.16 & 0.21 & 0.26 & 0.40 & 0.42 & 0.53 & 0.58 & 0.66 & 0.79 & 0.69 & 0.56 & 0.37 & 0.26 & 0.21 & 0.13 \\
\hline$(\mathrm{T} *-7)$ & 0.22 & 0.24 & 0.30 & \begin{tabular}{|l|}
0.43 \\
\end{tabular} & 0.46 & 0.51 & 0.54 & 0.59 & 0.67 & 0.62 & 0.56 & 0.40 & 0.32 & 0.27 & 0.16 \\
\hline$\left(T^{*}-8\right)$ & 0.13 & 0.19 & 0.32 & \begin{tabular}{|l|l|}
0.48 \\
\end{tabular} & 0.51 & 0.56 & 0.56 & 0.59 & 0.62 & 0.54 & 0.43 & 0.27 & 0.16 & 0.11 & 0.05 \\
\hline$\left(\mathrm{T}^{*}-9\right)$ & 0.30 & 0.32 & \begin{tabular}{|l|}
0.46 \\
\end{tabular} & 0.67 & 0.70 & 0.70 & 0.65 & 0.62 & 0.59 & 0.54 & 0.38 & 0.16 & 0.08 & 0.05 & 0.00 \\
\hline$\left(T^{*}-10\right)$ & \begin{tabular}{|l|}
0.54 \\
\end{tabular} & 0.43 & \begin{tabular}{|l|}
0.48 \\
\end{tabular} & 0.65 & 0.65 & 0.67 & 0.62 & 0.59 & 0.56 & 0.51 & 0.35 & 0.16 & 0.08 & \begin{tabular}{|l|}
0.00 \\
\end{tabular} & 0.00 \\
\hline$\left(\mathrm{T}^{*}-11\right)$ & \begin{tabular}{|l|}
0.75 \\
\end{tabular} & 0.56 & \begin{tabular}{|l|}
0.54 \\
\end{tabular} & \begin{tabular}{|l|}
0.65 \\
\end{tabular} & 0.65 & 0.59 & 0.54 & 0.51 & 0.48 & 0.46 & \begin{tabular}{|l|}
0.35 \\
\end{tabular} & 0.22 & 0.13 & \begin{tabular}{|l|}
0.08 \\
\end{tabular} & $\begin{array}{l}0.00 \\
\end{array}$ \\
\hline
\end{tabular}

\begin{tabular}{|c|c|c|c|c|c|c|c|c|c|c|c|c|c|c|c|}
\hline Danny (1997) & 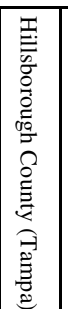 & 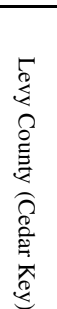 & 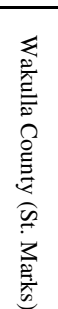 & 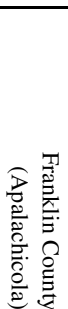 & 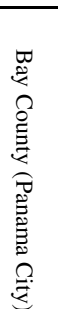 & 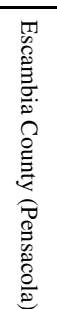 & 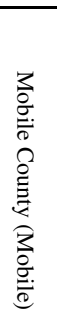 & 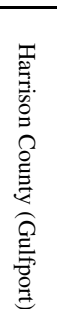 & 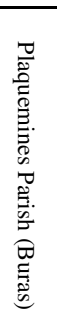 & 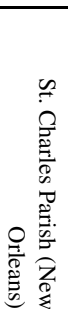 & 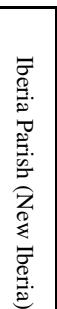 & 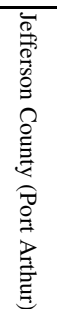 & 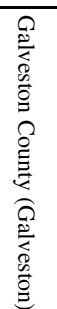 & 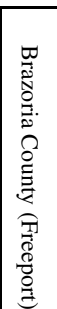 & $\begin{array}{r} \\
\\
\\
\end{array}$ \\
\hline $\mathrm{T}^{*}$ & \begin{tabular}{|l|}
0.02 \\
\end{tabular} & 0.04 & 0.10 & 0.10 & 0.16 & 0.63 & 0.63 & 0.63 & 0.14 & 0.12 & 0.09 & 0.05 & 0.03 & 0.03 & 0.01 \\
\hline$\left(T^{*}-1\right)$ & \begin{tabular}{|l|}
0.06 \\
\end{tabular} & 0.11 & 0.17 & 0.19 & 0.26 & 0.90 & 0.90 & 0.90 & 0.15 & 0.14 & 0.09 & 0.05 & 0.04 & 0.02 & 0.00 \\
\hline$\left(\mathrm{T}^{*}-2\right)$ & \begin{tabular}{|l|}
0.01 \\
\end{tabular} & 0.03 & 0.06 & 0.06 & 0.08 & 0.20 & 0.59 & 0.59 & 0.59 & 0.12 & 0.10 & 0.07 & 0.05 & 0.04 & 0.02 \\
\hline$\left(\mathrm{T}^{*}-3\right)$ & \begin{tabular}{|l|}
0.02 \\
\end{tabular} & 0.03 & 0.07 & 0.07 & 0.10 & 0.19 & 0.28 & 0.56 & 0.56 & 0.56 & 0.08 & 0.04 & 0.03 & 0.02 & 0.01 \\
\hline$\left(T^{*}-4\right)$ & \begin{tabular}{|l|}
0.03 \\
\end{tabular} & 0.05 & 0.08 & 0.08 & 0.10 & 0.14 & 0.20 & 0.26 & 0.56 & 0.56 & 0.09 & 0.05 & 0.03 & 0.02 & 0.01 \\
\hline$\left(\mathrm{T}^{*}-5\right)$ & \begin{tabular}{|l|}
0.02 \\
\end{tabular} & 0.03 & 0.04 & 0.04 & 0.05 & 0.08 & 0.11 & 0.14 & 0.31 & 0.31 & 0.05 & 0.03 & 0.02 & 0.01 & 0.01 \\
\hline$\left(\mathrm{T}^{*}-6\right)$ & \begin{tabular}{|l|}
0.00 \\
\end{tabular} & 0.00 & 0.00 & 0.00 & 0.00 & 0.00 & 0.00 & 0.00 & 0.00 & 0.00 & 0.00 & 0.00 & 0.00 & 0.00 & 0.00 \\
\hline$\left(T^{*}-7\right)$ & \begin{tabular}{|l|}
0.00 \\
\end{tabular} & 0.00 & 0.00 & 0.00 & 0.01 & 0.01 & 0.01 & 0.01 & 0.02 & 0.02 & 0.02 & 0.01 & 0.01 & 0.01 & 0.00 \\
\hline & & & & & & & & & & & & & & & \\
\hline *-9 & & & & & & & & & & & & & & & \\
\hline-10$)$ & & & & & & & & & & & & & & & \\
\hline & & & & & & & & & & & & & & & \\
\hline
\end{tabular}




\begin{tabular}{|c|c|c|c|c|c|c|c|c|c|c|c|c|c|c|c|}
\hline Allison (1995) & 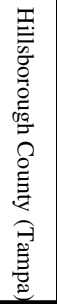 & 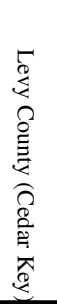 & 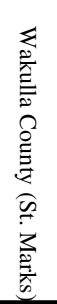 & 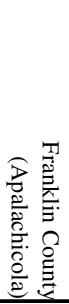 & 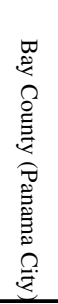 & 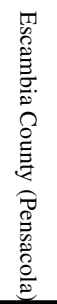 & 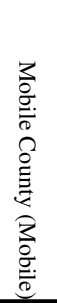 & 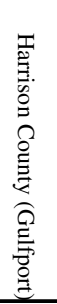 & 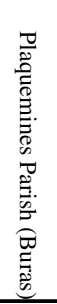 & 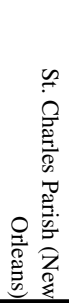 & 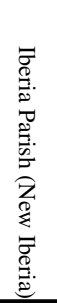 & 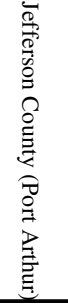 & 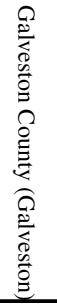 & 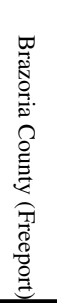 & 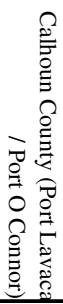 \\
\hline $\mathrm{T}^{*}$ & 0.03 & 0.13 & 0.33 & 0.45 & 0.45 & 0.23 & 0.03 & 0.00 & 0.00 & 0.00 & 0.00 & 0.00 & 0.00 & 0.00 & 0.00 \\
\hline$\left(\mathrm{T}^{*}-1\right)$ & 0.08 & 0.20 & 0.35 & 0.49 & 0.48 & 0.26 & 0.11 & 0.04 & 0.00 & 0.00 & 0.00 & 0.00 & 0.00 & 0.00 & 0.00 \\
\hline$\left(T^{*}-2\right)$ & 0.06 & 0.06 & 0.07 & 0.08 & 0.08 & 0.06 & 0.05 & 0.04 & 0.02 & 0.01 & 0.00 & 0.00 & 0.00 & 0.00 & 0.00 \\
\hline$(\mathrm{T} *-3)$ & 0.07 & 0.07 & 0.06 & 0.07 & 0.06 & 0.04 & 0.03 & 0.02 & 0.02 & 0.01 & 0.00 & 0.00 & 0.00 & 0.00 & 0.00 \\
\hline$\left(T^{*}-4\right)$ & 0.00 & 0.00 & 0.00 & 0.00 & 0.00 & 0.00 & 0.00 & 0.00 & 0.00 & 0.00 & 0.00 & 0.00 & 0.00 & 0.00 & 0.00 \\
\hline$\left(T^{*}-5\right)$ & 0.00 & 0.00 & 0.00 & 0.00 & 0.00 & 0.00 & 0.00 & 0.00 & 0.00 & 0.00 & 0.00 & 0.00 & 0.00 & 0.00 & 0.00 \\
\hline$\left(T^{*}-6\right)$ & & & & & & & & & & & & & & & \\
\hline$\left(T^{*}-7\right)$ & & & & & & & & & & & & & & & \\
\hline$\left(T^{*}-8\right)$ & & & & & & & & & & & & & & & \\
\hline$\left(T^{*}-9\right)$ & & & & & & & & & & & & & & & \\
\hline$(T *-10)$ & & & & & & & & & & & & & & & \\
\hline$(\mathrm{T} *-11)$ & & & & & & & & & & & & & & & \\
\hline
\end{tabular}

\begin{tabular}{|c|c|c|c|c|c|c|c|c|c|c|c|c|c|c|c|}
\hline Erin (1995) & 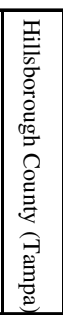 & 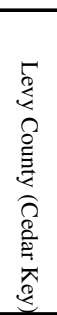 & 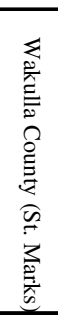 & 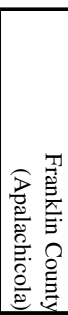 & 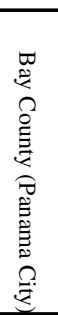 & 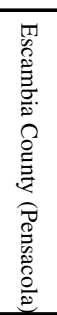 & 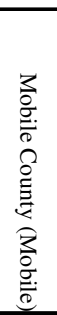 & 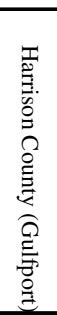 & 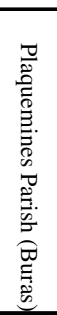 & 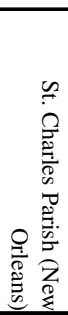 & 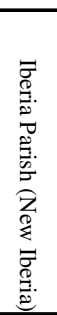 & 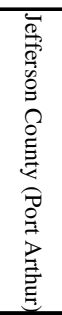 & 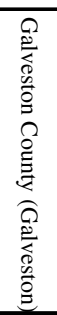 & 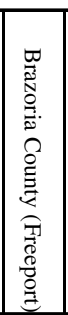 & 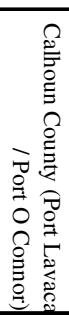 \\
\hline $\mathrm{T}^{*}$ & 0.00 & 0.00 & \begin{tabular}{|l|l|}
0.05 \\
\end{tabular} & 0.99 & 0.99 & 0.80 & 0.66 & 0.55 & 0.27 & 0.31 & 0.21 & 0.18 & 0.16 & 0.15 & 0.12 \\
\hline$\left(\mathrm{T}^{*}-1\right)$ & 0.00 & 0.05 & \begin{tabular}{|l|}
0.18 \\
\end{tabular} & 0.90 & 0.90 & 0.55 & 0.46 & 0.45 & 0.38 & 0.36 & 0.23 & 0.18 & 0.16 & 0.15 & 0.12 \\
\hline$\left(\mathrm{T}^{*}-2\right)$ & \begin{tabular}{|l|}
0.17 \\
\end{tabular} & 0.76 & 0.42 & \begin{tabular}{|l|}
0.65 \\
\end{tabular} & 0.55 & 0.38 & 0.29 & 0.26 & 0.20 & 0.20 & 0.15 & 0.12 & 0.08 & \begin{tabular}{|l|}
0.07 \\
\end{tabular} & 0.05 \\
\hline$\left(\mathrm{T}^{*}-3\right)$ & 0.21 & 0.21 & \begin{tabular}{|l|l|}
0.15 \\
\end{tabular} & \begin{tabular}{|l|}
0.15 \\
\end{tabular} & 0.13 & 0.09 & 0.07 & 0.05 & 0.03 & 0.04 & 0.03 & 0.01 & 0.01 & 0.00 & 0.00 \\
\hline$\left(T^{*}-4\right)$ & 0.04 & 0.03 & 0.02 & \begin{tabular}{|l|}
0.02 \\
\end{tabular} & 0.02 & 0.02 & 0.02 & 0.02 & 0.01 & 0.01 & 0.01 & 0.01 & 0.01 & 0.01 & 0.00 \\
\hline$(\mathrm{T} *-5)$ & 0.15 & 0.11 & 0.08 & \begin{tabular}{|l|}
0.08 \\
\end{tabular} & 0.08 & 0.07 & 0.06 & 0.06 & 0.06 & 0.06 & 0.05 & 0.04 & 0.04 & 0.03 & 0.02 \\
\hline$\left(\mathrm{T}^{*}-6\right)$ & 0.16 & 0.12 & 0.08 & \begin{tabular}{|l|}
0.09 \\
\end{tabular} & 0.08 & 0.07 & 0.07 & 0.07 & 0.07 & 0.06 & 0.06 & 0.04 & 0.04 & 0.03 & 0.02 \\
\hline$(\mathrm{T} *-7)$ & 0.20 & 0.16 & 0.14 & \begin{tabular}{|l|}
0.15 \\
\end{tabular} & 0.14 & 0.13 & 0.12 & 0.12 & 0.12 & 0.11 & 0.10 & 0.08 & 0.07 & 0.06 & 0.04 \\
\hline$(\mathrm{T} *-8)$ & 0.18 & 0.14 & 0.12 & \begin{tabular}{|l|}
0.13 \\
\end{tabular} & 0.13 & 0.12 & 0.11 & 0.11 & 0.12 & 0.11 & 0.10 & 0.08 & 0.07 & 0.06 & \begin{tabular}{|l|}
0.04 \\
\end{tabular} \\
\hline$\left(\mathrm{T}^{*}-9\right)$ & 0.22 & 0.18 & \begin{tabular}{|l|l|}
0.15 \\
\end{tabular} & 0.16 & 0.16 & 0.15 & 0.15 & 0.15 & 0.16 & 0.15 & 0.13 & 0.11 & 0.09 & 0.07 & 0.05 \\
\hline$\left(\mathrm{T}^{*}-10\right)$ & \begin{tabular}{|l|l|}
0.16 \\
\end{tabular} & 0.13 & \begin{tabular}{|l|l|}
0.11 \\
\end{tabular} & \begin{tabular}{|l|}
0.13 \\
\end{tabular} & 0.13 & 0.12 & 0.12 & 0.13 & 0.14 & 0.13 & 0.12 & 0.10 & 0.09 & \begin{tabular}{|l|}
0.09 \\
\end{tabular} & 0.09 \\
\hline$\left(\mathrm{T}^{*}-11\right)$ & 0.14 & 0.11 & \begin{tabular}{|l|}
0.10 \\
\end{tabular} & \begin{tabular}{|l|}
0.10 \\
\end{tabular} & 0.10 & 0.10 & 0.10 & 0.10 & 0.10 & 0.10 & \begin{tabular}{|l|}
0.09 \\
\end{tabular} & 0.06 & 0.06 & 0.05 & 0.04 \\
\hline
\end{tabular}

\begin{tabular}{|c|c|c|c|c|c|c|c|c|c|c|c|c|c|c|c|}
\hline Opal (1995) & 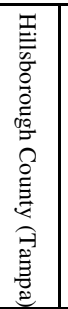 & 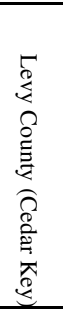 & 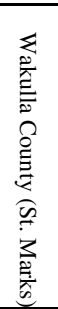 & 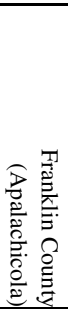 & 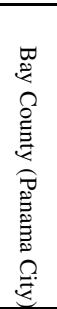 & 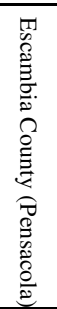 & 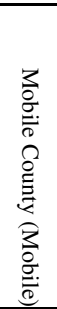 & 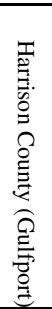 & 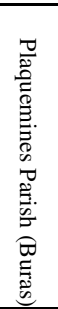 & 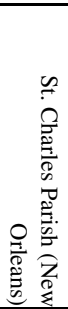 & 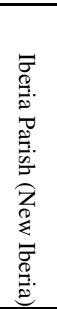 & 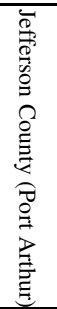 & 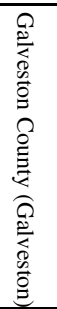 & 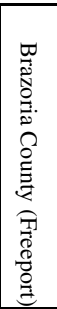 & 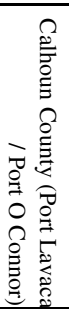 \\
\hline $\mathrm{T}^{*}$ & 0.00 & 0.00 & 0.60 & 0.92 & 1.84 & 2.80 & 1.76 & 0.60 & 0.28 & 0.00 & 0.00 & 0.00 & 0.00 & 0.00 & 0.00 \\
\hline$\left(T^{*}-1\right)$ & \begin{tabular}{|l|}
0.07 \\
\end{tabular} & 0.22 & 0.65 & 0.83 & 1.08 & 1.40 & 1.15 & 0.86 & 0.76 & 0.29 & 0.00 & 0.00 & 0.00 & 0.00 & 0.00 \\
\hline$\left(\mathrm{T}^{*}-2\right)$ & \begin{tabular}{|l|}
0.30 \\
\end{tabular} & 0.46 & 0.61 & 0.67 & 0.70 & 0.67 & 0.58 & 0.49 & 0.52 & 0.36 & 0.18 & 0.06 & 0.00 & 0.00 & 0.00 \\
\hline$(\mathrm{T} *-3)$ & \begin{tabular}{|l|}
0.24 \\
\end{tabular} & 0.37 & 0.49 & 0.54 & 0.56 & 0.54 & 0.46 & 0.39 & 0.41 & 0.29 & 0.15 & 0.05 & 0.00 & 0.00 & 0.00 \\
\hline$\left(T^{*}-4\right)$ & \begin{tabular}{|l|}
0.18 \\
\end{tabular} & 0.24 & 0.30 & 0.34 & 0.36 & 0.40 & 0.38 & 0.38 & 0.42 & 0.34 & 0.22 & 0.08 & 0.04 & 0.00 & 0.00 \\
\hline & 16 & 0.20 & 0.24 & 0.26 & 0.28 & 0.32 & 0.32 & 0.34 & 0.38 & 0.34 & 0.30 & 0.16 & 0.12 & 0.10 & 0.04 \\
\hline$\left(T^{*}-6\right)$ & 18 & 0.20 & 0.24 & 0.26 & 0.26 & 0.30 & 0.30 & 0.30 & 0.34 & 0.30 & 0.24 & 0.12 & 0.08 & 0.06 & 0.00 \\
\hline$\left(T^{*}-7\right)$ & \begin{tabular}{|l|}
0.16 \\
\end{tabular} & 0.16 & 0.18 & 0.20 & 0.22 & 0.22 & 0.24 & 0.24 & 0.28 & 0.26 & 0.24 & 0.18 & 0.16 & 0.14 & 0.10 \\
\hline$\left(T^{*}-8\right)$ & \begin{tabular}{|l|}
0.04 \\
\end{tabular} & 0.04 & 0.04 & 0.05 & 0.05 & 0.07 & 0.07 & 0.09 & 0.12 & 0.12 & 0.12 & 0.12 & 0.14 & 0.14 & 0.14 \\
\hline & & 0.00 & 0.00 & 0.02 & 0.02 & 0.03 & 0.04 & 0.04 & 0.06 & 0.06 & 0.07 & 0.07 & 0.09 & 0.10 & 0.1 \\
\hline$\left(\mathrm{T}^{*}-10\right)$ & \begin{tabular}{|l|}
0.03 \\
\end{tabular} & 0.03 & 0.03 & 0.04 & 0.04 & 0.04 & 0.04 & 0.05 & 0.07 & 0.06 & 0.06 & 0.05 & 0.06 & 0.06 & 0.06 \\
\hline$\left(\mathrm{T}^{*}-11\right)$ & \begin{tabular}{|l|}
0.02 \\
\end{tabular} & 0.02 & 0.02 & 0.03 & 0.03 & 0.04 & 0.04 & 0.05 & 0.06 & 0.06 & 0.06 & 0.06 & 0.07 & 0.07 & 0.07 \\
\hline
\end{tabular}




\begin{tabular}{|c|c|c|c|c|c|c|c|c|c|c|c|c|c|c|c|}
\hline Andrew (1992) & 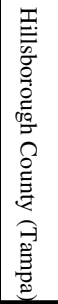 & 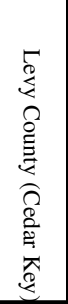 & 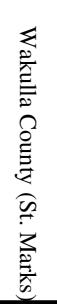 & 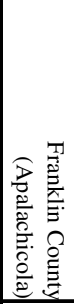 & 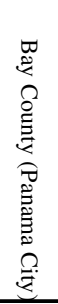 & 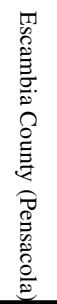 & 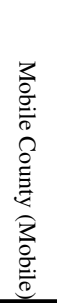 & 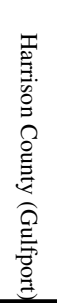 & 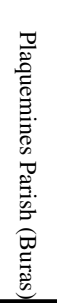 & 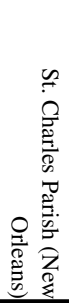 & 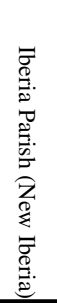 & 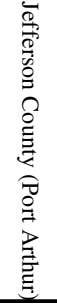 & 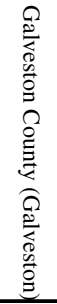 & 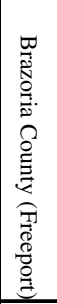 & 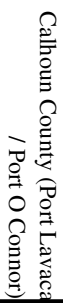 \\
\hline $\mathrm{T}^{*}$ & 0.00 & \begin{tabular}{|l|}
0.00 \\
\end{tabular} & 0.24 & 0.24 & 0.28 & 0.44 & 0.52 & 0.60 & 2.56 & 2.64 & 3.04 & 1.20 & 0.44 & 0.20 & 0.08 \\
\hline$\left(\mathrm{T}^{*}-1\right)$ & 0.00 & 0.00 & 0.00 & 0.16 & 0.24 & 0.36 & 0.52 & 0.64 & 1.75 & 1.47 & 1.99 & 1.11 & 0.76 & 0.60 & 0.40 \\
\hline$\left(\mathrm{T}^{*}-2\right)$ & 0.00 & \begin{tabular}{|l|}
0.00 \\
\end{tabular} & 0.00 & 0.24 & 0.28 & 0.44 & 0.56 & 0.72 & 1.99 & 1.43 & 1.51 & 0.95 & 0.80 & 0.68 & 0.52 \\
\hline$(\mathrm{T} *-3)$ & 0.00 & \begin{tabular}{|l|}
0.00 \\
\end{tabular} & 0.19 & 0.27 & 0.30 & 0.50 & 0.61 & 0.76 & 1.22 & 0.95 & 0.88 & 0.69 & 0.65 & 0.61 & 0.50 \\
\hline$\left(T^{*}-4\right)$ & 0.15 & \begin{tabular}{|l|}
0.22 \\
\end{tabular} & 0.34 & 0.41 & 0.49 & 0.60 & 0.67 & 0.75 & 0.86 & 0.79 & 0.71 & 0.52 & 0.49 & 0.41 & 0.34 \\
\hline$\left(\mathrm{T}^{*}-5\right)$ & 0.00 & \begin{tabular}{|l|}
0.00 \\
\end{tabular} & 0.22 & 0.30 & 0.37 & 0.60 & 0.67 & 0.79 & 0.97 & 0.86 & 0.79 & 0.64 & 0.56 & 0.49 & 0.37 \\
\hline$\left(T^{*}-6\right)$ & 0.22 & \begin{tabular}{|l|}
0.15 \\
\end{tabular} & 0.22 & 0.34 & 0.41 & 0.56 & 0.64 & 0.71 & 0.90 & 0.79 & 0.75 & 0.60 & 0.56 & 0.49 & 0.41 \\
\hline$(\mathrm{T} *-7)$ & 0.67 & \begin{tabular}{|l|}
0.35 \\
\end{tabular} & 0.28 & 0.42 & 0.39 & 0.42 & 0.42 & 0.46 & 0.56 & 0.49 & 0.49 & 0.42 & 0.42 & 0.39 & 0.35 \\
\hline$\left(T^{*}-8\right)$ & 0.67 & \begin{tabular}{|l|}
0.35 \\
\end{tabular} & 0.28 & 0.42 & 0.39 & 0.42 & 0.42 & 0.46 & 0.56 & 0.49 & 0.49 & 0.42 & 0.42 & 0.39 & 0.35 \\
\hline$\left(T^{*}-9\right)$ & 0.70 & \begin{tabular}{|l|}
0.46 \\
\end{tabular} & 0.32 & 0.42 & 0.39 & 0.39 & 0.39 & 0.42 & 0.49 & 0.46 & 0.46 & 0.42 & 0.42 & 0.42 & 0.39 \\
\hline$\left(T^{*}-10\right)$ & 0.55 & \begin{tabular}{|l|}
0.39 \\
\end{tabular} & 0.32 & 0.39 & 0.36 & 0.36 & 0.36 & 0.36 & 0.42 & 0.39 & 0.36 & 0.32 & 0.32 & 0.29 & 0.26 \\
\hline$(\mathrm{T} *-11)$ & 0.39 & \begin{tabular}{|l|}
0.30 \\
\end{tabular} & 0.23 & 0.27 & 0.27 & 0.25 & 0.23 & 0.23 & 0.27 & 0.25 & 0.20 & 0.16 & 0.16 & 0.16 & 0.14 \\
\hline
\end{tabular}




\section{Probability Transition Matrices}

\begin{tabular}{|c|c|c|c|c|c|c|}
\hline $\mathbf{T} * \backslash \mathbf{T}$ & $\mathbf{0}$ & $\mathbf{1}$ & $\mathbf{2}$ & $\mathbf{3}$ & $\mathbf{4}$ & $\mathbf{5}$ \\
\hline $\mathbf{0}$ & $100 \%$ & $0 \%$ & $0 \%$ & $0 \%$ & $0 \%$ & $0 \%$ \\
\hline $\mathbf{0 - 0 . 5}$ & $97 \%$ & $3 \%$ & $0 \%$ & $0 \%$ & $0 \%$ & $0 \%$ \\
\hline $\mathbf{0 . 5}-\mathbf{1}$ & $78 \%$ & $22 \%$ & $0 \%$ & $0 \%$ & $0 \%$ & $0 \%$ \\
\hline $\mathbf{1 - 1 . 5}$ & $80 \%$ & $15 \%$ & $5 \%$ & $0 \%$ & $0 \%$ & $0 \%$ \\
\hline $\mathbf{1 . 5}-\mathbf{2}$ & $63 \%$ & $0 \%$ & $25 \%$ & $13 \%$ & $0 \%$ & $0 \%$ \\
\hline $\mathbf{2 - 2 . 5}$ & $67 \%$ & $5 \%$ & $20 \%$ & $8 \%$ & $0 \%$ & $0 \%$ \\
\hline $\mathbf{2 . 5}-\mathbf{3}$ & $22 \%$ & $0 \%$ & $0 \%$ & $78 \%$ & $0 \%$ & $0 \%$ \\
\hline $\mathbf{3 - 3 . 5}$ & $0 \%$ & $0 \%$ & $0 \%$ & $80 \%$ & $20 \%$ & $0 \%$ \\
\hline $\mathbf{3 . 5}-\mathbf{4}$ & $0 \%$ & $0 \%$ & $0 \%$ & $80 \%$ & $20 \%$ & $0 \%$ \\
\hline $\mathbf{4 - 4 . 5}$ & N/A & N/A & N/A & N/A & N/A & N/A \\
\hline $\mathbf{4 . 5}-\mathbf{5}$ & N/A & N/A & N/A & N/A & N/A & N/A \\
\hline
\end{tabular}

\begin{tabular}{|c|c|c|c|c|c|c|c|c|c|c|c|}
\hline$\left(T^{*}-1\right) \backslash T^{*}$ & $\mathbf{0}$ & $0-0.5$ & 0.5 - 1 & $1-1.5$ & $1.5-2$ & $2-2.5$ & $2.5-3$ & $3-3.5$ & $3.5-4$ & $4-4.5$ & $4.5-5$ \\
\hline 0 & $98 \%$ & $2 \%$ & $0 \%$ & $0 \%$ & $0 \%$ & $0 \%$ & $0 \%$ & $0 \%$ & $0 \%$ & $0 \%$ & $0 \%$ \\
\hline $0-0.5$ & $37 \%$ & $60 \%$ & $3 \%$ & $0 \%$ & $0 \%$ & $0 \%$ & $0 \%$ & $0 \%$ & $0 \%$ & $0 \%$ & $0 \%$ \\
\hline 0.5 - 1 & $9 \%$ & $30 \%$ & $45 \%$ & $12 \%$ & $3 \%$ & $0 \%$ & $0 \%$ & $0 \%$ & $0 \%$ & $0 \%$ & $0 \%$ \\
\hline $1-1.5$ & $0 \%$ & $6 \%$ & $25 \%$ & $13 \%$ & $38 \%$ & $6 \%$ & $13 \%$ & $0 \%$ & $0 \%$ & $0 \%$ & $0 \%$ \\
\hline $1.5-2$ & $0 \%$ & $9 \%$ & $9 \%$ & $9 \%$ & $9 \%$ & $9 \%$ & $36 \%$ & $9 \%$ & $9 \%$ & $0 \%$ & $0 \%$ \\
\hline $2-2.5$ & $0 \%$ & $0 \%$ & $0 \%$ & $0 \%$ & $28 \%$ & $20 \%$ & $52 \%$ & $0 \%$ & $0 \%$ & $0 \%$ & $0 \%$ \\
\hline $2.5-3$ & $0 \%$ & $0 \%$ & $0 \%$ & $0 \%$ & $0 \%$ & $35 \%$ & $15 \%$ & $15 \%$ & $35 \%$ & $0 \%$ & $0 \%$ \\
\hline $3-3.5$ & N/A & N/A & N/A & N/A & N/A & N/A & N/A & N/A & N/A & N/A & N/A \\
\hline $3.5-4$ & N/A & N/A & N/A & N/A & N/A & N/A & N/A & N/A & N/A & N/A & N/A \\
\hline $4-4.5$ & N/A & N/A & N/A & N/A & N/A & N/A & N/A & N/A & N/A & N/A & N/A \\
\hline $4.5-5$ & N/A & N/A & N/A & N/A & N/A & N/A & N/A & N/A & N/A & N/A & N/A \\
\hline
\end{tabular}

\begin{tabular}{|c|c|c|c|c|c|c|c|c|c|c|c|}
\hline$\left(\mathbf{T}^{*-2}\right) \backslash\left(\mathbf{T}^{*-1}\right)$ & $\mathbf{0}$ & $\mathbf{0 - 0 . 5}$ & $\mathbf{0 . 5}-\mathbf{1}$ & $\mathbf{1}-\mathbf{1 . 5}$ & $\mathbf{1 . 5}-\mathbf{2}$ & $\mathbf{2}-\mathbf{2 . 5}$ & $\mathbf{2 . 5}-\mathbf{3}$ & $\mathbf{3}-\mathbf{3 . 5}$ & $\mathbf{3 . 5}-\mathbf{4}$ & $\mathbf{4}-\mathbf{4 . 5}$ & $\mathbf{4 . 5}-\mathbf{5}$ \\
\hline $\mathbf{0}$ & $99 \%$ & $1 \%$ & $0 \%$ & $0 \%$ & $0 \%$ & $0 \%$ & $0 \%$ & $0 \%$ & $0 \%$ & $0 \%$ & $0 \%$ \\
\hline $\mathbf{0 - 0 . 2 5}$ & $14 \%$ & $83 \%$ & $2 \%$ & $0 \%$ & $0 \%$ & $0 \%$ & $0 \%$ & $0 \%$ & $0 \%$ & $0 \%$ & $0 \%$ \\
\hline $\mathbf{0 . 2 5} \mathbf{- 0 . 5}$ & $4 \%$ & $85 \%$ & $12 \%$ & $0 \%$ & $0 \%$ & $0 \%$ & $0 \%$ & $0 \%$ & $0 \%$ & $0 \%$ & $0 \%$ \\
\hline $\mathbf{0 . 5} \mathbf{- 0 . 7 5}$ & $0 \%$ & $18 \%$ & $64 \%$ & $18 \%$ & $0 \%$ & $0 \%$ & $0 \%$ & $0 \%$ & $0 \%$ & $0 \%$ & $0 \%$ \\
\hline $\mathbf{0 . 7 5 - 1}$ & $7 \%$ & $7 \%$ & $50 \%$ & $29 \%$ & $7 \%$ & $0 \%$ & $0 \%$ & $0 \%$ & $0 \%$ & $0 \%$ & $0 \%$ \\
\hline $\mathbf{1 - 1 . 5}$ & $0 \%$ & $0 \%$ & $17 \%$ & $39 \%$ & $33 \%$ & $6 \%$ & $6 \%$ & $0 \%$ & $0 \%$ & $0 \%$ & $0 \%$ \\
\hline $\mathbf{1 . 5}-\mathbf{2}$ & $0 \%$ & $0 \%$ & $0 \%$ & $0 \%$ & $57 \%$ & $29 \%$ & $14 \%$ & $0 \%$ & $0 \%$ & $0 \%$ & $0 \%$ \\
\hline $\mathbf{2 - 3}$ & N/A & N/A & N/A & N/A & N/A & N/A & N/A & N/A & N/A & N/A & N/A \\
\hline $\mathbf{3 - 4}$ & N/A & N/A & N/A & N/A & N/A & N/A & N/A & N/A & N/A & N/A & N/A \\
\hline $\mathbf{4 - 5}$ & N/A & N/A & N/A & N/A & N/A & N/A & N/A & N/A & N/A & N/A & N/A \\
\hline
\end{tabular}

\begin{tabular}{|c|c|c|c|c|c|c|c|c|c|c|}
\hline$\left(\mathbf{T}^{*-3}\right) \backslash\left(\mathbf{T}^{*-2}\right)$ & $\mathbf{0}$ & $\mathbf{0}-\mathbf{0 . 2 5}$ & $\mathbf{0 . 2 5}-\mathbf{0 . 5}$ & $\mathbf{0 . 5}-\mathbf{0 . 7 5}$ & $\mathbf{0 . 7 5}-\mathbf{1}$ & $\mathbf{1}-\mathbf{1 . 5}$ & $\mathbf{1 . 5}-\mathbf{2}$ & $\mathbf{2}-\mathbf{3}$ & $\mathbf{3 - 4}$ & $\mathbf{4}-\mathbf{5}$ \\
\hline $\mathbf{0}$ & $97 \%$ & $3 \%$ & $0 \%$ & $0 \%$ & $0 \%$ & $0 \%$ & $0 \%$ & $0 \%$ & $0 \%$ & $0 \%$ \\
\hline $\mathbf{0 - 0 . 2 5}$ & $17 \%$ & $69 \%$ & $7 \%$ & $5 \%$ & $2 \%$ & $0 \%$ & $0 \%$ & $0 \%$ & $0 \%$ & $0 \%$ \\
\hline $\mathbf{0 . 2 5} \mathbf{- 0 . 5}$ & $3 \%$ & $25 \%$ & $44 \%$ & $25 \%$ & $3 \%$ & $0 \%$ & $0 \%$ & $0 \%$ & $0 \%$ & $0 \%$ \\
\hline $\mathbf{0 . 5} \mathbf{- 0 . 7 5}$ & $0 \%$ & $4 \%$ & $21 \%$ & $46 \%$ & $29 \%$ & $0 \%$ & $0 \%$ & $0 \%$ & $0 \%$ & $0 \%$ \\
\hline $\mathbf{0 . 7 5 - 1}$ & $0 \%$ & $0 \%$ & $0 \%$ & $22 \%$ & $22 \%$ & $50 \%$ & $6 \%$ & $0 \%$ & $0 \%$ & $0 \%$ \\
\hline $\mathbf{1 - 1 . 5}$ & $0 \%$ & $0 \%$ & $0 \%$ & $0 \%$ & $0 \%$ & $60 \%$ & $40 \%$ & $0 \%$ & $0 \%$ & $0 \%$ \\
\hline $\mathbf{1 . 5}-\mathbf{2}$ & N/A & N/A & N/A & N/A & N/A & N/A & N/A & N/A & N/A & N/A \\
\hline $\mathbf{2 - 3}$ & N/A & N/A & N/A & N/A & N/A & N/A & N/A & N/A & N/A & N/A \\
\hline $\mathbf{3 - 4}$ & N/A & N/A & N/A & N/A & N/A & N/A & N/A & N/A & N/A & N/A \\
\hline $\mathbf{4 - 5}$ & N/A & N/A & N/A & N/A & N/A & N/A & N/A & N/A & N/A & N/A \\
\hline
\end{tabular}




\begin{tabular}{|c|c|c|c|c|c|c|c|c|c|c|}
\hline$\left(T^{*}-4\right) \backslash\left(T^{*}-3\right)$ & $\mathbf{0}$ & $0-0.25$ & $0.25-0.5$ & $0.5-0.75$ & $0.75-1$ & $1-1.5$ & $1.5-2$ & $2-3$ & $3-4$ & $4-5$ \\
\hline $\mathbf{0}$ & $81 \%$ & $19 \%$ & $0 \%$ & $0 \%$ & $0 \%$ & $0 \%$ & $0 \%$ & $0 \%$ & $0 \%$ & $0 \%$ \\
\hline 0 - 0.25 & $10 \%$ & $84 \%$ & $6 \%$ & $0 \%$ & $0 \%$ & $0 \%$ & $0 \%$ & $0 \%$ & $0 \%$ & $0 \%$ \\
\hline $0.25-0.5$ & $0 \%$ & $11 \%$ & $59 \%$ & $30 \%$ & $0 \%$ & $0 \%$ & $0 \%$ & $0 \%$ & $0 \%$ & $0 \%$ \\
\hline $0.5-0.75$ & $0 \%$ & $4 \%$ & $15 \%$ & $48 \%$ & $30 \%$ & $4 \%$ & $0 \%$ & $0 \%$ & $0 \%$ & $0 \%$ \\
\hline $0.75-1$ & $0 \%$ & $0 \%$ & $0 \%$ & $0 \%$ & $60 \%$ & $40 \%$ & $0 \%$ & $0 \%$ & $0 \%$ & $0 \%$ \\
\hline $1-1.5$ & $0 \%$ & $0 \%$ & $0 \%$ & $0 \%$ & $11 \%$ & $89 \%$ & $0 \%$ & $0 \%$ & $0 \%$ & $0 \%$ \\
\hline $1.5-2$ & N/A & N/A & N/A & N/A & N/A & N/A & N/A & N/A & N/A & N/A \\
\hline $2-3$ & N/A & N/A & N/A & N/A & N/A & N/A & N/A & N/A & N/A & N/A \\
\hline $3-4$ & N/A & N/A & N/A & N/A & N/A & N/A & N/A & N/A & N/A & N/A \\
\hline $4-5$ & N/A & N/A & N/A & N/A & N/A & N/A & N/A & N/A & N/A & N/A \\
\hline
\end{tabular}

\begin{tabular}{|c|c|c|c|c|c|c|c|c|c|c|}
\hline$\left(T^{*}-5\right) \backslash\left(T^{*}-4\right)$ & $\overline{0}$ & $0-0.25$ & $0.25-0.5$ & $0.5-0.75$ & $0.75-1$ & $1-1.5$ & $1.5-2$ & $2-3$ & $3-4$ & $4-5$ \\
\hline $\mathbf{0}$ & $97 \%$ & $3 \%$ & $0 \%$ & $0 \%$ & $0 \%$ & $0 \%$ & $0 \%$ & $0 \%$ & $0 \%$ & $0 \%$ \\
\hline $0-0.25$ & $24 \%$ & $72 \%$ & $4 \%$ & $0 \%$ & $0 \%$ & $0 \%$ & $0 \%$ & $0 \%$ & $0 \%$ & $0 \%$ \\
\hline $0.25-0.5$ & $0 \%$ & $6 \%$ & $82 \%$ & $12 \%$ & $0 \%$ & $0 \%$ & $0 \%$ & $0 \%$ & $0 \%$ & $0 \%$ \\
\hline $0.5-0.75$ & $0 \%$ & $4 \%$ & $19 \%$ & $67 \%$ & $11 \%$ & $0 \%$ & $0 \%$ & $0 \%$ & $0 \%$ & $0 \%$ \\
\hline $0.75-1$ & $0 \%$ & $0 \%$ & $0 \%$ & $23 \%$ & $41 \%$ & $36 \%$ & $0 \%$ & $0 \%$ & $0 \%$ & $0 \%$ \\
\hline $1-1.5$ & $0 \%$ & $0 \%$ & $0 \%$ & $0 \%$ & $75 \%$ & $25 \%$ & $0 \%$ & $0 \%$ & $0 \%$ & $0 \%$ \\
\hline $1.5-2$ & N/A & N/A & N/A & N/A & N/A & N/A & N/A & N/A & N/A & N/A \\
\hline $2-3$ & N/A & N/A & N/A & N/A & N/A & N/A & N/A & N/A & N/A & N/A \\
\hline $3-4$ & N/A & N/A & N/A & N/A & N/A & N/A & N/A & N/A & N/A & N/A \\
\hline $4-5$ & N/A & N/A & N/A & N/A & N/A & N/A & N/A & N/A & N/A & N/A \\
\hline
\end{tabular}

\begin{tabular}{|c|c|c|c|c|c|c|c|c|c|c|}
\hline$\left(\mathbf{T}^{*-6)} \backslash\left(\mathbf{T}^{*-5}\right)\right.$ & $\mathbf{0}$ & $\mathbf{0 - 0 . 2 5}$ & $\mathbf{0 . 2 5}-\mathbf{0 . 5}$ & $\mathbf{0 . 5}-\mathbf{0 . 7 5}$ & $\mathbf{0 . 7 5 - 1}$ & $\mathbf{1}-\mathbf{1 . 5}$ & $\mathbf{1 . 5}-\mathbf{2}$ & $\mathbf{2 - 3}$ & $\mathbf{3 - 4}$ & $\mathbf{4 - 5}$ \\
\hline $\mathbf{0}$ & $75 \%$ & $23 \%$ & $3 \%$ & $0 \%$ & $0 \%$ & $0 \%$ & $0 \%$ & $0 \%$ & $0 \%$ & $0 \%$ \\
\hline $\mathbf{0 - 0 . 2 5}$ & $6 \%$ & $92 \%$ & $2 \%$ & $0 \%$ & $0 \%$ & $0 \%$ & $0 \%$ & $0 \%$ & $0 \%$ & $0 \%$ \\
\hline $\mathbf{0 . 2 5 - 0 . 5}$ & $0 \%$ & $23 \%$ & $68 \%$ & $10 \%$ & $0 \%$ & $0 \%$ & $0 \%$ & $0 \%$ & $0 \%$ & $0 \%$ \\
\hline $\mathbf{0 . 5}-\mathbf{0 . 7 5}$ & $0 \%$ & $0 \%$ & $6 \%$ & $61 \%$ & $25 \%$ & $8 \%$ & $0 \%$ & $0 \%$ & $0 \%$ & $0 \%$ \\
\hline $\mathbf{0 . 7 5 - 1}$ & $0 \%$ & $0 \%$ & $0 \%$ & $7 \%$ & $93 \%$ & $0 \%$ & $0 \%$ & $0 \%$ & $0 \%$ & $0 \%$ \\
\hline $\mathbf{1 - 1 . 5}$ & $0 \%$ & $0 \%$ & $0 \%$ & $0 \%$ & $0 \%$ & $100 \%$ & $0 \%$ & $0 \%$ & $0 \%$ & $0 \%$ \\
\hline $\mathbf{1 . 5}-\mathbf{2}$ & N/A & N/A & N/A & N/A & N/A & N/A & N/A & N/A & N/A & N/A \\
\hline $\mathbf{2 - 3}$ & N/A & N/A & N/A & N/A & N/A & N/A & N/A & N/A & N/A & N/A \\
\hline $\mathbf{3 - 4}$ & N/A & N/A & N/A & N/A & N/A & N/A & N/A & N/A & N/A & N/A \\
\hline $\mathbf{4 - 5}$ & N/A & N/A & N/A & N/A & N/A & N/A & N/A & N/A & N/A & N/A \\
\hline
\end{tabular}

\begin{tabular}{|c|c|c|c|c|c|c|c|c|c|c|}
\hline$(T *-7) \backslash(T *-6)$ & $\mathbf{0}$ & $0-0.25$ & $0.25-0.5$ & $0.5-0.75$ & $0.75-1$ & $1-1.5$ & $1.5-2$ & $2-3$ & $3-4$ & $4-5$ \\
\hline 0 & $85 \%$ & $15 \%$ & $0 \%$ & $0 \%$ & $0 \%$ & $0 \%$ & $0 \%$ & $0 \%$ & $0 \%$ & $0 \%$ \\
\hline $0-0.25$ & $12 \%$ & $79 \%$ & $9 \%$ & $0 \%$ & $0 \%$ & $0 \%$ & $0 \%$ & $0 \%$ & $0 \%$ & $0 \%$ \\
\hline $0.25-0.5$ & $0 \%$ & $11 \%$ & $64 \%$ & $22 \%$ & $2 \%$ & $0 \%$ & $0 \%$ & $0 \%$ & $0 \%$ & $0 \%$ \\
\hline $0.5-0.75$ & $0 \%$ & $3 \%$ & $3 \%$ & $79 \%$ & $14 \%$ & $0 \%$ & $0 \%$ & $0 \%$ & $0 \%$ & $0 \%$ \\
\hline $0.75-1$ & $0 \%$ & $0 \%$ & $0 \%$ & $23 \%$ & $69 \%$ & $8 \%$ & $0 \%$ & $0 \%$ & $0 \%$ & $0 \%$ \\
\hline $1-2$ & N/A & N/A & N/A & N/A & N/A & N/A & N/A & N/A & N/A & N/A \\
\hline $2-3$ & N/A & N/A & N/A & N/A & N/A & N/A & N/A & N/A & N/A & N/A \\
\hline $3-4$ & N/A & N/A & N/A & N/A & N/A & N/A & N/A & N/A & N/A & N/A \\
\hline $4-5$ & N/A & N/A & N/A & N/A & N/A & N/A & N/A & N/A & N/A & N/A \\
\hline
\end{tabular}




\begin{tabular}{|c|c|c|c|c|c|c|c|c|c|}
\hline$\left(\mathbf{T}^{*-8}\right) \backslash\left(\mathbf{T}^{*-7}\right)$ & $\mathbf{0}$ & $\mathbf{0 - 0 . 2 5}$ & $\mathbf{0 . 2 5}-\mathbf{0 . 5}$ & $\mathbf{0 . 5} \mathbf{- 0 . 7 5}$ & $\mathbf{0 . 7 5 - 1}$ & $\mathbf{1 ~ - 2}$ & $\mathbf{2 - 3}$ & $\mathbf{3 - 4}$ & $\mathbf{4 - 5}$ \\
\hline $\mathbf{0}$ & $79 \%$ & $21 \%$ & $0 \%$ & $0 \%$ & $0 \%$ & $0 \%$ & $0 \%$ & $0 \%$ & $0 \%$ \\
\hline $\mathbf{0 - 0 . 2 5}$ & $6 \%$ & $84 \%$ & $9 \%$ & $0 \%$ & $0 \%$ & $0 \%$ & $0 \%$ & $0 \%$ & $0 \%$ \\
\hline $\mathbf{0 . 2 5 - 0 . 5}$ & $0 \%$ & $5 \%$ & $72 \%$ & $23 \%$ & $0 \%$ & $0 \%$ & $0 \%$ & $0 \%$ & $0 \%$ \\
\hline $\mathbf{0 . 5 - 0 . 7 5}$ & $0 \%$ & $0 \%$ & $13 \%$ & $63 \%$ & $25 \%$ & $0 \%$ & $0 \%$ & $0 \%$ & $0 \%$ \\
\hline $\mathbf{0 . 7 5 - 1}$ & $0 \%$ & $0 \%$ & $0 \%$ & $0 \%$ & $100 \%$ & $0 \%$ & $0 \%$ & $0 \%$ & $0 \%$ \\
\hline $\mathbf{1 - 2}$ & N/A & N/A & N/A & N/A & N/A & N/A & N/A & N/A & N/A \\
\hline $\mathbf{2 - 3}$ & N/A & N/A & N/A & N/A & N/A & N/A & N/A & N/A & N/A \\
\hline $\mathbf{3 - 4}$ & N/A & N/A & N/A & N/A & N/A & N/A & N/A & N/A & N/A \\
\hline $\mathbf{4 - 5}$ & N/A & N/A & N/A & N/A & N/A & N/A & N/A & N/A & N/A \\
\hline
\end{tabular}

\begin{tabular}{|c|c|c|c|c|c|c|c|c|c|}
\hline$\left(\mathbf{T}^{*-9}\right) \backslash\left(\mathbf{T}^{*-8}\right)$ & $\mathbf{0}$ & $\mathbf{0 - 0 . 2 5}$ & $\mathbf{0 . 2 5}-\mathbf{0 . 5}$ & $\mathbf{0 . 5} \mathbf{- 0 . 7 5}$ & $\mathbf{0 . 7 5}-\mathbf{1}$ & $\mathbf{1}-\mathbf{2}$ & $\mathbf{2}-\mathbf{3}$ & $\mathbf{3 - 4}$ & $\mathbf{4}-\mathbf{5}$ \\
\hline $\mathbf{0}$ & $65 \%$ & $35 \%$ & $0 \%$ & $0 \%$ & $0 \%$ & $0 \%$ & $0 \%$ & $0 \%$ & $0 \%$ \\
\hline $\mathbf{0 - 0 . 2 5}$ & $4 \%$ & $89 \%$ & $6 \%$ & $0 \%$ & $0 \%$ & $0 \%$ & $0 \%$ & $0 \%$ & $0 \%$ \\
\hline $\mathbf{0 . 2 5} \mathbf{- 0 . 5}$ & $0 \%$ & $12 \%$ & $81 \%$ & $7 \%$ & $0 \%$ & $0 \%$ & $0 \%$ & $0 \%$ & $0 \%$ \\
\hline $\mathbf{0 . 5} \mathbf{- 0 . 7 5}$ & $0 \%$ & $0 \%$ & $9 \%$ & $80 \%$ & $11 \%$ & $0 \%$ & $0 \%$ & $0 \%$ & $0 \%$ \\
\hline $\mathbf{0 . 7 5 - 1}$ & $0 \%$ & $0 \%$ & $0 \%$ & $100 \%$ & $0 \%$ & $0 \%$ & $0 \%$ & $0 \%$ & $0 \%$ \\
\hline $\mathbf{1 - 2}$ & N/A & N/A & N/A & N/A & N/A & N/A & N/A & N/A & N/A \\
\hline $\mathbf{2 - 3}$ & N/A & N/A & N/A & N/A & N/A & N/A & N/A & N/A & N/A \\
\hline $\mathbf{3 - 4}$ & N/A & N/A & N/A & N/A & N/A & N/A & N/A & N/A & N/A \\
\hline $\mathbf{4 - 5}$ & N/A & N/A & N/A & N/A & N/A & N/A & N/A & N/A & N/A \\
\hline
\end{tabular}

\begin{tabular}{|c|c|c|c|c|c|c|c|c|c|}
\hline$\left(T^{*}-10\right) \backslash\left(T^{*}-9\right)$ & $\mathbf{0}$ & $0-0.25$ & $0.25-0.5$ & $0.5-0.75$ & $0.75-1$ & $1-2$ & $2-3$ & $3-4$ & $4-5$ \\
\hline $\mathbf{0}$ & $80 \%$ & $20 \%$ & $0 \%$ & $0 \%$ & $0 \%$ & $0 \%$ & $0 \%$ & $0 \%$ & $0 \%$ \\
\hline $0-0.25$ & $10 \%$ & $85 \%$ & $5 \%$ & $0 \%$ & $0 \%$ & $0 \%$ & $0 \%$ & $0 \%$ & $0 \%$ \\
\hline $0.25-0.5$ & $0 \%$ & $0 \%$ & $79 \%$ & $21 \%$ & $0 \%$ & $0 \%$ & $0 \%$ & $0 \%$ & $0 \%$ \\
\hline $0.5-0.75$ & $0 \%$ & $0 \%$ & $13 \%$ & $84 \%$ & $3 \%$ & $0 \%$ & $0 \%$ & $0 \%$ & $0 \%$ \\
\hline $0.75-1$ & N/A & N/A & N/A & N/A & N/A & N/A & N/A & N/A & N/A \\
\hline $1-2$ & N/A & N/A & N/A & N/A & N/A & N/A & N/A & N/A & N/A \\
\hline $2-3$ & N/A & N/A & N/A & N/A & N/A & $\mathrm{N} / \mathrm{A}$ & N/A & N/A & N/A \\
\hline $3-4$ & N/A & N/A & N/A & N/A & N/A & N/A & N/A & N/A & N/A \\
\hline $4-5$ & N/A & N/A & N/A & N/A & N/A & N/A & N/A & N/A & N/A \\
\hline
\end{tabular}

\begin{tabular}{|c|c|c|c|c|c|c|c|c|c|}
\hline$\left(\mathbf{T}^{*-11}\right) \backslash\left(\mathbf{T}^{*-10}\right)$ & $\mathbf{0}$ & $\mathbf{0 - 0 . 2 5}$ & $\mathbf{0 . 2 5}-\mathbf{0 . 5}$ & $\mathbf{0 . 5} \mathbf{- 0 . 7 5}$ & $\mathbf{0 . 7 5 - 1}$ & $\mathbf{1 - 2}$ & $\mathbf{2 - 3}$ & $\mathbf{3 - 4}$ & $\mathbf{4 - 5}$ \\
\hline $\mathbf{0}$ & $89 \%$ & $11 \%$ & $0 \%$ & $0 \%$ & $0 \%$ & $0 \%$ & $0 \%$ & $0 \%$ & $0 \%$ \\
\hline $\mathbf{0 - 0 . 2 5}$ & $16 \%$ & $65 \%$ & $19 \%$ & $0 \%$ & $0 \%$ & $0 \%$ & $0 \%$ & $0 \%$ & $0 \%$ \\
\hline $\mathbf{0 . 2 5} \mathbf{- 0 . 5}$ & $0 \%$ & $3 \%$ & $58 \%$ & $40 \%$ & $0 \%$ & $0 \%$ & $0 \%$ & $0 \%$ & $0 \%$ \\
\hline $\mathbf{0 . 5} \mathbf{- 0 . 7 5}$ & $0 \%$ & $0 \%$ & $12 \%$ & $88 \%$ & $0 \%$ & $0 \%$ & $0 \%$ & $0 \%$ & $0 \%$ \\
\hline $\mathbf{0 . 7 5 - 1}$ & N/A & N/A & N/A & N/A & N/A & N/A & N/A & N/A & N/A \\
\hline $\mathbf{1 - 2}$ & N/A & N/A & N/A & N/A & N/A & N/A & N/A & N/A & N/A \\
\hline $\mathbf{2 - 3}$ & N/A & N/A & N/A & N/A & N/A & N/A & N/A & N/A & N/A \\
\hline $\mathbf{3 - 4}$ & N/A & N/A & N/A & N/A & N/A & N/A & N/A & N/A & N/A \\
\hline $\mathbf{4 - 5}$ & N/A & N/A & N/A & N/A & N/A & N/A & N/A & N/A & N/A \\
\hline
\end{tabular}




\section{Dynamic Programming Results}

\begin{tabular}{|c|c|c|c|c|}
\hline$\theta\left(T^{*}\right)$ & C_EV(T*) & $\mathbf{E}[\mathbf{V}(\mathbf{T})]$ & $\begin{array}{l}\text { Optimal } \\
\text { Decision }\end{array}$ & $\mathbf{V}\left(\mathbf{T}^{*}\right)$ \\
\hline $\mathbf{0}$ & 290 & 0 & Wait & 0 \\
\hline $0-0.5$ & 339 & 47 & Wait & 47 \\
\hline $0.5-1$ & 387 & 368 & Wait & 368 \\
\hline $1-1.5$ & 416 & 593 & Evac & 416 \\
\hline $1.5-2$ & 446 & 3599 & Evac & 446 \\
\hline $2-2.5$ & 486 & 2659 & Evac & 486 \\
\hline $2.5-3$ & 526 & 11857 & Evac & 526 \\
\hline $3-3.5$ & 551 & 17954 & Evac & 551 \\
\hline $3.5-4$ & 575 & 17954 & Evac & 575 \\
\hline $4-4.5$ & 583 & N/A & N/A & N/A \\
\hline $4.5-5$ & 590 & N/A & N/A & N/A \\
\hline
\end{tabular}

\begin{tabular}{|c|c|c|c|c|}
\hline$\theta\left(T^{*}-1\right)$ & C_EV(T*-1) & $\mathbf{E}\left[\mathbf{V}\left(\mathbf{T}^{*}\right)\right]$ & $\begin{array}{l}\text { Optimal } \\
\text { Decision }\end{array}$ & $V(T *-1)$ \\
\hline $\mathbf{0}$ & 254 & 1 & Wait & 1 \\
\hline $0-0.5$ & 296 & 40 & Wait & 40 \\
\hline $0.5-1$ & 339 & 246 & Wait & 246 \\
\hline $1-1.5$ & 363 & 410 & Evac & 363 \\
\hline $1.5-2$ & 388 & 454 & Evac & 388 \\
\hline $2-2.5$ & 422 & 495 & Evac & 422 \\
\hline $2.5-3$ & 456 & 533 & Evac & 456 \\
\hline $3-3.5$ & 477 & N/A & N/A & N/A \\
\hline $3.5-4$ & 497 & N/A & N/A & N/A \\
\hline $4-4.5$ & 504 & N/A & N/A & N/A \\
\hline $4.5-5$ & 510 & N/A & N/A & N/A \\
\hline
\end{tabular}

\begin{tabular}{|c|c|c|c|c|}
\hline $\boldsymbol{\theta}\left(\mathbf{T}^{*-2}\right)$ & $\mathbf{C} \_\mathbf{E V}\left(\mathbf{T}^{*-2}\right)$ & $\mathbf{E}\left[\mathbf{V}\left(\mathbf{T}^{*-1}\right)\right]$ & $\begin{array}{c}\text { Optimal } \\
\text { Decision }\end{array}$ & $\mathbf{V}\left(\mathbf{T}^{*-2}\right)$ \\
\hline $\mathbf{0}$ & 226 & 1 & Wait & 1 \\
\hline $\mathbf{0 . 2 5}$ & 244 & 39 & Wait & 39 \\
\hline $\mathbf{0 . 5}$ & 263 & 62 & Wait & 62 \\
\hline $\mathbf{0 . 5} \mathbf{- 0 . 7 5}$ & 282 & 230 & Wait & 230 \\
\hline $\mathbf{0 . 7 5} \mathbf{- 1}$ & 301 & 257 & Wait & 257 \\
\hline $\mathbf{1 - 1 . 5}$ & 322 & 360 & Evac & 322 \\
\hline $\mathbf{1 . 5}-\mathbf{2}$ & 343 & 407 & Evac & 343 \\
\hline $\mathbf{2 - 3}$ & 401 & N/A & N/A & N/A \\
\hline $\mathbf{3 - 4}$ & 436 & N/A & N/A & N/A \\
\hline $\mathbf{4 - 5}$ & 447 & N/A & N/A & N/A \\
\hline
\end{tabular}

\begin{tabular}{|c|c|c|c|c|}
\hline $\boldsymbol{\theta}\left(\mathbf{T}^{*-3}\right)$ & $\mathbf{C} \_\mathbf{E V}\left(\mathbf{T}^{*-3}\right)$ & $\mathbf{E}\left[\mathbf{V}\left(\mathbf{T}^{*-2}\right)\right]$ & $\begin{array}{c}\text { Optimal } \\
\text { Decision }\end{array}$ & $\mathbf{V}\left(\mathbf{T}^{*-3}\right)$ \\
\hline $\mathbf{0}$ & 198 & 2 & Wait & 2 \\
\hline $\mathbf{0 . 2 5}$ & 214 & 47 & Wait & 47 \\
\hline $\mathbf{0 . 2 5} \mathbf{- 0 . 5}$ & 231 & 102 & Wait & 102 \\
\hline $\mathbf{0 . 5} \mathbf{- 0 . 7 5}$ & 247 & 195 & Wait & 195 \\
\hline $\mathbf{0 . 7 5 - 1}$ & 264 & 288 & Evac & 264 \\
\hline $\mathbf{1 - 1 . 5}$ & 281 & 330 & Evac & 281 \\
\hline $\mathbf{1 . 5} \mathbf{- 2}$ & 299 & N/A & N/A & N/A \\
\hline $\mathbf{3}$ & 347 & N/A & N/A & N/A \\
\hline $\mathbf{4}$ & 377 & N/A & N/A & N/A \\
\hline $\mathbf{5}$ & 386 & N/A & N/A & N/A \\
\hline
\end{tabular}




\begin{tabular}{|c|c|c|c|c|}
\hline$\theta\left(T^{*-4}\right)$ & C_EV $\left(T^{*} * 4\right)$ & $\mathrm{E}[\mathrm{V}(\mathrm{T} *-\mathbf{3})]$ & $\begin{array}{l}\text { Optimal } \\
\text { Decision }\end{array}$ & $V(T *-4)$ \\
\hline $\mathbf{0}$ & 235 & 11 & Wait & 11 \\
\hline 0.25 & 255 & 47 & Wait & 47 \\
\hline $0.25-0.5$ & 274 & 124 & Wait & 124 \\
\hline $0.5-0.75$ & 294 & 199 & Wait & 199 \\
\hline $0.75-1$ & 313 & 271 & Wait & 271 \\
\hline 1.5 & 328 & 279 & Wait & 279 \\
\hline $1.5-2$ & 343 & N/A & N/A & N/A \\
\hline 3 & 383 & N/A & N/A & N/A \\
\hline 4 & 408 & N/A & N/A & N/A \\
\hline 5 & 416 & N/A & N/A & N/A \\
\hline
\end{tabular}

\begin{tabular}{|c|c|c|c|c|}
\hline$\theta\left(T^{*-5}\right)$ & C_EV(T*-5) & $\mathrm{E}[\mathrm{V}(\mathrm{T} *-4)]$ & $\begin{array}{l}\text { Optimal } \\
\text { Decision }\end{array}$ & $\mathrm{V}(\mathrm{T} *-5)$ \\
\hline $\mathbf{0}$ & 225 & 12 & Wait & 12 \\
\hline 0.25 & 244 & 41 & Wait & 41 \\
\hline $0.25-0.5$ & 263 & 128 & Wait & 128 \\
\hline $0.5-0.75$ & 281 & 188 & Wait & 188 \\
\hline $0.75-1$ & 300 & 258 & Wait & 258 \\
\hline $1-1.5$ & 314 & 273 & Wait & 273 \\
\hline $1.5-2$ & 327 & N/A & N/A & N/A \\
\hline 3 & 364 & N/A & N/A & N/A \\
\hline 4 & 387 & N/A & N/A & N/A \\
\hline 5 & 393 & N/A & N/A & N/A \\
\hline
\end{tabular}

\begin{tabular}{|c|c|c|c|c|}
\hline$\theta(T *-6)$ & C_EV(T*-6) & $\mathrm{E}[\mathrm{V}(\mathrm{T} *-5)]$ & $\begin{array}{l}\text { Optimal } \\
\text { Decision }\end{array}$ & $V(T *-6)$ \\
\hline $\mathbf{0}$ & 219 & 22 & Wait & 22 \\
\hline 0.25 & 237 & 41 & Wait & 41 \\
\hline 0.5 & 255 & 115 & Wait & 115 \\
\hline $0.5-0.75$ & 273 & 209 & Wait & 209 \\
\hline $0.75-1$ & 292 & 253 & Wait & 253 \\
\hline $1-1.5$ & 304.12 & 272.96 & Wait & 273 \\
\hline $1.5-2$ & 317 & N/A & N/A & N/A \\
\hline 3 & 351 & N/A & N/A & N/A \\
\hline 4 & 373 & N/A & N/A & N/A \\
\hline 5 & 379 & N/A & N/A & N/A \\
\hline
\end{tabular}

\begin{tabular}{|c|c|c|c|c|}
\hline $\boldsymbol{\theta}\left(\mathbf{T}^{*-7}\right)$ & $\mathbf{C} \mathbf{E V}\left(\mathbf{T}^{*-7}\right)$ & $\mathbf{E}\left[\mathbf{V}\left(\mathbf{T}^{*-6}\right)\right]$ & $\begin{array}{c}\text { Optimal } \\
\text { Decision }\end{array}$ & $\mathbf{V}(\mathbf{T} * \mathbf{- 7})$ \\
\hline $\mathbf{0}$ & 213 & 25 & Wait & 25 \\
\hline $\mathbf{0 . 2 5}$ & 231 & 45 & Wait & 45 \\
\hline $\mathbf{0 . 2 5} \mathbf{- 0 . 5}$ & 249 & 131 & Wait & 131 \\
\hline $\mathbf{0 . 5} \mathbf{0 . 7 5}$ & 267 & 206 & Wait & 206 \\
\hline $\mathbf{. 7 5} \mathbf{- 1}$ & 284 & 244 & Wait & 244 \\
\hline $\mathbf{2}$ & 308 & N/A & N/A & N/A \\
\hline $\mathbf{3}$ & 341 & N/A & N/A & N/A \\
\hline $\mathbf{4}$ & 361 & N/A & N/A & N/A \\
\hline $\mathbf{5}$ & 367 & N/A & N/A & N/A \\
\hline
\end{tabular}




\begin{tabular}{|c|c|c|c|c|}
\hline $\boldsymbol{\theta}\left(\mathbf{T}^{*-8}\right)$ & $\mathbf{C} \_\mathbf{E V}\left(\mathbf{T}^{*-8}\right)$ & $\mathbf{E}\left[\mathbf{V}\left(\mathbf{T}^{*-7}\right)\right]$ & $\begin{array}{c}\text { Optimal } \\
\text { Decision }\end{array}$ & $\mathbf{V}\left(\mathbf{T}^{*-8}\right)$ \\
\hline $\mathbf{0}$ & 303 & 29 & Wait & 29 \\
\hline $\mathbf{0 . 2 5}$ & 328 & 52 & Wait & 52 \\
\hline $\mathbf{0 . 5}$ & 353 & 144 & Wait & 144 \\
\hline $\mathbf{0 . 7 5}$ & 378 & 206 & Wait & 206 \\
\hline $\mathbf{1}$ & 404 & 244 & Wait & 244 \\
\hline $\mathbf{2}$ & 435 & N/A & N/A & N/A \\
\hline $\mathbf{3}$ & 479 & N/A & N/A & N/A \\
\hline $\mathbf{4}$ & 505 & N/A & N/A & N/A \\
\hline $\mathbf{5}$ & 513 & N/A & N/A & N/A \\
\hline
\end{tabular}

\begin{tabular}{|c|c|c|c|c|}
\hline$\theta\left(T^{*}-9\right)$ & C_EV(T*-9) & $\mathbf{E}\left[\mathbf{V}\left(\mathbf{T}^{*} \boldsymbol{-} \mathbf{8}\right)\right]$ & $\begin{array}{l}\text { Optimal } \\
\text { Decision }\end{array}$ & $V(T *-9)$ \\
\hline $\mathbf{0}$ & 299 & 37 & Wait & 37 \\
\hline 0.25 & 324 & 57 & Wait & 57 \\
\hline 0.5 & 349 & 138 & Wait & 138 \\
\hline 0.75 & 374 & 205 & Wait & 205 \\
\hline 1 & 399 & 206 & Wait & 206 \\
\hline 2 & 429 & N/A & N/A & N/A \\
\hline 3 & 472 & N/A & N/A & N/A \\
\hline 4 & 497 & N/A & N/A & N/A \\
\hline 5 & 505 & N/A & N/A & N/A \\
\hline
\end{tabular}

\begin{tabular}{|c|c|c|c|c|}
\hline $\boldsymbol{\theta}\left(\mathbf{T}^{*-10}\right)$ & $\mathbf{C} \_\mathbf{E V}\left(\mathbf{T}^{*-10}\right)$ & $\mathbf{E}\left[\mathbf{V}\left(\mathbf{T}^{*-9}\right)\right]$ & $\begin{array}{c}\text { Optimal } \\
\text { Decision }\end{array}$ & $\mathbf{V}\left(\mathbf{T}^{*-10}\right)$ \\
\hline $\mathbf{0}$ & 294 & 41 & Wait & 41 \\
\hline $\mathbf{0 . 2 5}$ & 318 & 59 & Wait & 59 \\
\hline $\mathbf{0 . 5}$ & 343 & 152 & Wait & 152 \\
\hline $\mathbf{0 . 7 5}$ & 367 & 196 & Wait & 196 \\
\hline $\mathbf{1}$ & 392 & N/A & N/A & N/A \\
\hline $\mathbf{2}$ & 421 & N/A & N/A & N/A \\
\hline $\mathbf{3}$ & 461 & N/A & N/A & N/A \\
\hline $\mathbf{4}$ & 486 & N/A & N/A & N/A \\
\hline $\mathbf{5}$ & 493 & N/A & N/A & N/A \\
\hline
\end{tabular}

\begin{tabular}{|c|c|c|c|c|}
\hline$\theta(T *-11)$ & C_EV(T*-11) & $E[V(T *-10)]$ & $\begin{array}{l}\text { Optimal } \\
\text { Decision }\end{array}$ & $V(T *-11)$ \\
\hline $\mathbf{0}$ & 290 & 43 & Wait & 43 \\
\hline 0.25 & 314 & 74 & Wait & 74 \\
\hline 0.5 & 338 & 167 & Wait & 167 \\
\hline 0.75 & 363 & 191 & Wait & 191 \\
\hline 1 & 387 & N/A & N/A & N/A \\
\hline 2 & 415 & N/A & N/A & N/A \\
\hline 3 & 454 & N/A & N/A & N/A \\
\hline 4 & 478 & N/A & N/A & N/A \\
\hline 5 & 485 & N/A & N/A & N/A \\
\hline
\end{tabular}


VITA

\section{JEFFREY ROBERT CZAJKOWSKI}

$\begin{array}{ll}\text { August 9, } 1973 & \text { Born, Cleveland, Ohio } \\ 1995 & \begin{array}{l}\text { B.S., Industrial Management and Political Science } \\ \text { Carnegie Mellon University } \\ \text { Pittsburgh, Pennsylvania }\end{array} \\ & \text { Coopers \& Lybrand Consulting } \\ \text { New York, New York } & \\ 1995-1997 & \text { JP Morgan } \\ & \text { New York, New York } \\ 1997-2001 & \text { E.P.A. STAR Fellow } \\ & \text { M.S., Environmental and Urban Systems } \\ 2002-2004 & \text { Florida International University } \\ & \text { Miami, FL } \\ & \text { Economics Instructor } \\ & \begin{array}{l}\text { Florida International University } \\ \text { Miami, FL }\end{array} \\ & \begin{array}{l}\text { Doctoral Candidate in Economics } \\ \text { Florida International University } \\ \text { Miami, FL }\end{array} \\ & \text { Research Assistant } \\ 2004-2005 & \begin{array}{l}\text { Florida International University } \\ \text { International Hurricane Research Center } \\ \text { Miami, FL }\end{array} \\ & \text { FIU Dissertation Fellow }\end{array}$

\section{PUBLICATIONS AND PRESENTATIONS}

Czajkowski, J., 2007. "Is It Time to Go Yet? Dynamically Modeling Hurricane Evacuations", Florida International University International Hurricane Research Center Technical Report. 
Czajkowski, J., 2007. "Willingness-To-Pay Bias in Dichotomous Choice Surveys", revision requested, Land Economics.

$21^{\text {st }}$ Annual Governor's Hurricane Conference, Fort Lauderdale, FL (May, 2007)

$1^{\text {st }}$ Annual Symposium on Disasters, The Roosevelt Institution at Tulane University, New Orleans, LA (April, 2007)

Florida International University Graduate Scholarly Forum, Miami, FL (April, 2007)

Florida Hurricane Alliance Workshop, Miami, FL (March, 2007)

Ohio Wesleyan University, Department of Economics, Delaware, OH (February, 2007)

Washington College, Department of Economics, Chestertown, MD (February, 2007)

E.P.A. National Center for Environmental Economics, Washington D.C. (February, 2007)

Austin College, Department of Economics, Sherman, TX (February, 2007)

Florida International University, Department of Economics, Miami, FL (October, 2006)

Camp Resources XIV, Wilmington, N.C. (August, 2006)

Florida Hurricane Alliance Workshop, Miami, FL (March, 2006)

E.P.A Science Forum, Washington D.C. (May 2005)

Florida Coastal Everglades Long Term Ecological Research Meeting, Miami (March, 2005)

Florida International University Graduate Scholarly Forum, Miami, FL (March, 2005)

E.P.A. Graduate Fellowship Conference, Washington D.C. (October, 2004) 